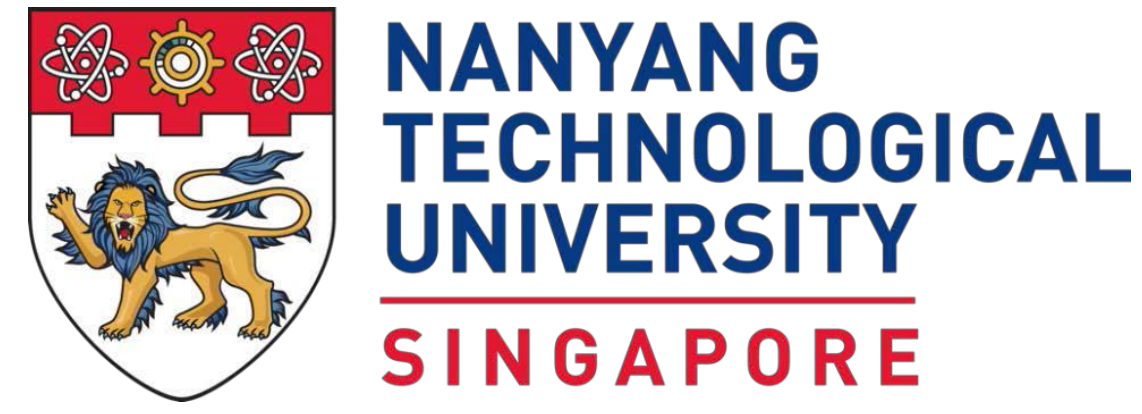

\title{
APPLICATION OF PLANAR HELIX SLOW-WAVE STRUCTURE IN BACKWARD-WAVE OSCILLATORS
}

Mookkannoor Muraleedharan Nair Ajith Kumar

SCHOOL OF ELECTRICAL \& ELECTRONIC ENGINEERING 2019 


\section{APPLICATION OF PLANAR HELIX SLOW-WAVE STRUCTURE IN BACKWARD-WAVE OSCILLATORS}

\section{Mookkannoor Muraleedharan Nair Ajith Kumar}

School of Electrical \& Electronic Engineering

A thesis submitted to the Nanyang Technological University in partial fulfillment of the requirement for the degree of Doctor of Philosophy 


\section{Statement of Originality}

I hereby certify that the work embodied in this thesis is the result of original research, is free of plagiarized materials, and has not been submitted for a higher degree to any other University or Institution.

03-06-2020

Date

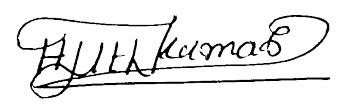

Mookkannoor Muraleedharan Nair Ajith Kumar 


\section{Supervisor Declaration Statement}

I have reviewed the content and presentation style of this thesis and declare it is free of plagiarism and of sufficient grammatical clarity to be examined. To the best of my knowledge, the research and writing are those of the candidate except as acknowledged in the Author Attribution Statement. I confirm that the investigations were conducted in accord with the ethics policies and integrity standards of Nanyang Technological University and that the research data are presented honestly and without prejudice.

3rd June 2020

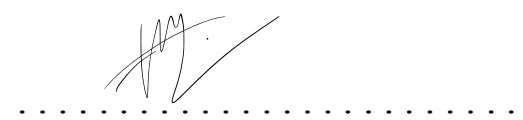

Date

Assoc Prof Lee Yee Hui 


\section{Authorship Attribution Statement}

This thesis contains material from 4 papers published in the following peer-reviewed journals where I was the first author.

Parts of Chapter 3 are published as A. Kumar. M. M., S. Aditya and C. Chua, "Interaction Impedance for Space Harmonics of Circular Helix Using Simulations," IEEE Transactions on Electron Devices, vol. 64, no. 4, pp. 1868-1872, April 2017. doi: 10.1109/TED.2017.2669332

The contributions of the co-authors are as follows:

- $\mathrm{A} /$ Prof Sheel Aditya provided the initial project direction.

- I prepared the manuscript and A/Prof Sheel Aditya edited the manuscript.

- Dr Cier Siang Chua identified the problem statement and give directions for solving it.

- I carried out the simulations and compared the results from simulation with analysis.

- The works were carried out under the supervision of A/Prof Sheel Aditya. The results were verified by Dr Cier Siang Chua.

- $\mathrm{A} /$ Prof Sheel Aditya provided guidance in the interpretation of the results.

Parts of Chapter 4 are published as

1) A. Kumar M. M and S. Aditya, "Simplified Tape-Helix Analysis of the Planar Helix Slow Wave Structure with Straight-Edge Connections," in IEEE Transactions on Electron Devices, vol. 65, no. 6, pp. 2280-2286, June 2018. doi: 10.1109/TED.2018.2797928

2) M. M. A. Kumar and S. Aditya, "Simplified tape-helix analysis of planar helix slowwave structure using effective dielectric constant method," 2017 Eighteenth International Vacuum Electronics Conference (IVEC), London, 2017, pp. 1-2. doi: 10.1109/IVEC.2017.8289700

3) M. M. A. Kumar, S. Aditya and C. Zhao, "Transmission characteristics of planar tapehelix: Simulation and measurements," 2018 IEEE International Vacuum Electronics 
Conference (IVEC), Monterey, CA, USA, 2018, pp. 343-344. doi: 10.1109/IVEC.2018.8391517

4) M. M. A. Kumar and S. Aditya, "Dispersion characteristics of planar tape-helix using effective dielectric constant method," 2018 IEEE International Vacuum Electronics Conference (IVEC), Monterey, CA, USA, 2018, pp. 381-382. doi: 10.1109/IVEC.2018.8391683

The contributions of the co-authors are as follows:

- A/Prof Sheel Aditya identified the research gap and provided direction for the work.

- I prepared the manuscript and A/Prof Sheel Aditya edited the manuscript.

- I carried out the analysis using tape-helix approximation and A/Prof Sheel Aditya verified it.

- I compared the results from numerical analysis with those from simulation under the guidance of A/Prof Sheel Aditya.

- A/Prof Sheel Aditya provide guidance in the interpretation of the results.

- Dr Zhao Chen helped in fabricating the PH-SEC immersed in a homogeneous medium for experimental validation of the analysis.

Parts of Chapter 5 are published as M. M. Ajith Kumar, S. Aditya and S. Wang, "A WBand Backward-Wave Oscillator Based on Planar Helix Slow Wave Structure," in IEEE Transactions on Electron Devices, vol. 65, no. 11, pp. 5097-5102, Nov. 2018. doi: 10.1109/TED.2018.287178.

The contributions of the co-authors are as follows:

- $\mathrm{A} /$ Prof Sheel Aditya provided guidance and direction for this work.

- I prepared the manuscript and A/Prof Sheel Aditya edited the manuscript.

- Dr Wang Shaomeng helped in solving the problems during modelling and simulation of the oscillator.

- I carried out the modelling, and simulation of the oscillator. Besides I carried out the fabrication and measurement of the prototype. 
- A/Prof Sheel Aditya and Dr Wang Shaomeng provided guidance and help in the interpretation of the results.

Parts of Chapter 6 are published as A. Kumar M. M and S. Aditya, "Vacuum Electronic Two-Beam Oscillator-Amplifier," in IEEE Transactions on Plasma Science, vol. 45, no. 8, pp. 2260-2267, Aug. 2017. doi: 10.1109/TPS.2017.2726109

The contributions of the co-authors are as follows:

- I proposed the idea for the work and discussed it with A/Prof Sheel Aditya.

- I prepared the manuscript and A/Prof Sheel Aditya edited the manuscript.

- I carried out the simulation of the oscillator-amplifier and compared the results with those of conventional BWO. A/Prof Sheel Aditya verified it.

- A/Prof Sheel Aditya provided interpretation of the results.

03-06-2020

Date

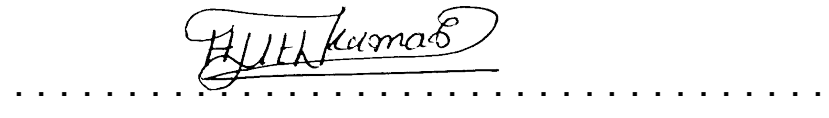

Mookkannoor Muraleedharan Nair Ajith Kumar 



\section{Acknowledgement}

First and foremost, I would like thank God for giving me patience, strength, and knowledge to complete this research.

I would like to express my sincere gratitude to my Ph.D. supervisor Associate Professor Sheel Aditya for his guidance during past 4-years of my Ph.D. study in NTU. He motivated me to do research in this topic. His deep knowledge, and experience in the subject helped me a lot to deal with the complications during this research. He helped me in understanding the fundamentals, analytical methods and experimental methods for this work. He encouraged and motivated me to improve my writing and presentation skills. His understanding nature and patience helped me to complete this work smoothly.

I also like to express my sincere gratitude to my second supervisor Associate Professor Lee Yee Hui for her help in the difficult and crucial final stage of my Ph.D. She has taken the responsibility as my supervisor since prof Sheel Aditya retired from NTU before completing my oral defence.

I extend my gratitude to Dr. Ciersiang Chua, Dr. Zhao Chen, and Dr. Wang Shaomeng for their help in simulations and experiments. Dr. Zhao Chen taught me the basics of travelling-wave tube simulations and encouraged me to do this research. Dr. Ciersiang Chua, and Dr. Wang Shaomeng helped me whenever I encountered some simulation problems.

I would also like to express my gratitude to the School of Electrical and Electronic Engineering, Nanyang Technological University, for offering me a peaceful working atmosphere and scholarship for this research. I also extend my gratitude to every staff and friend in the Satellite Research Centre, NTU for their help during my Ph.D. Besides, I would like to thank all my friends at NTU.

Last but not least, I would like to express sincere thanks to my father Muraleedharan Nair, my mother Indhiramma, my brother Arun, and my wife Anupama for their understanding and encouragement. 


\section{Abstract}

Travelling-wave tubes (TWTs) are one of the most popular device among vacuum electron devices. TWTs can be used as amplifiers, constituting travelling-wave tube amplifiers (TWTAs), or as oscillators, constituting backward-wave oscillators (BWOs). A BWO is one of the most reliable and spectrally pure voltage-tuneable high frequency oscillator. A slow-wave structure (SWS) is a very important component in TWTs. It becomes challenging to build and operate TWTs at millimetre-wave frequencies (30 - 300 $\mathrm{GHz}$ ) since the dimensions of the SWSs and the beam tunnel that accommodates the electron beam reduce as the frequency of operation increases.

Microfabrication techniques are a possible solution to achieve the required small sizes. Hence research on microfabrication-compatible SWSs is very important. Planar helix slow-wave structure with straight-edge connections (PH-SEC) is a planar counterpart of circular helix SWS which is one of the most popular SWS for TWTs. The PH-SEC is readily amenable to microfabrication. The PH-SEC has been studied for TWTA applications in the past few years. In this thesis we investigate its potential for application in BWOs.

First, the Fourier decomposition method is used to evaluate, more accurately than before, the interaction impedance for the fundamental and non-fundamental space harmonics of the SWSs such as the circular helix. Accurate evaluation of the interaction impedance for different space harmonics in a SWS is an important step for the design of TWTs. Results are presented for the variation of interaction impedance inside the SWS with frequency and position.

Field-theory based analysis is the fastest method to determine the dispersion characteristics of SWSs. The results from such an analysis can be used for the initial selection of dimensions of the PH-SEC for a TWT with given target specifications. In the second investigation, the dispersion characteristics as well as the interaction impedance of the PH-SEC are obtained using the tape-helix approximation. The analysis is simplified by combining the tape-helix analysis and the effective dielectric constant (EDC) method. Moreover, a PH-SEC immersed in a homogeneous dielectric medium has been fabricated 
and tested. The measured phase velocity compares very well with that obtained from the analytical results.

The third investigation presents simulation results for the design and performance of a BWO that operates at $\mathrm{W}$-band and uses a microfabrication-compatible $\mathrm{PH}-\mathrm{SEC}$ as the SWS. The oscillator is designed to operate with a beam voltage varying from $7 \mathrm{KV}$ to 11 $\mathrm{KV}$ and a beam current of $20 \mathrm{~mA}$. The particle-in-cell (PIC) simulation results show that the oscillator frequency tunes from $86.9 \mathrm{GHz}$ to $100 \mathrm{GHz}$ with a tuneable bandwidth of $14 \%$. Further, a scaled version of the PH-SEC operating at X-band is also fabricated and measured. The measured S-parameters and the phase velocity match very well with the simulation results.

Finally, a new technique is proposed to improve the efficiency of the conventional BWOs. The technique uses an additional electron beam inside the conventional BWO; the additional beam is synchronized with the fundamental forward-wave space harmonic and provides amplification like a TWTA. The technique is illustrated using a circular helix SWS at $\mathrm{Ku}$-band. It is shown that the proposed technique improves the DC-to-RF conversion efficiency of the conventional BWO by a factor ranging from three to six depending on the beam configuration. The same technique for efficiency improvement is also illustrated using the PH-SEC.

The studies reported in this thesis show that the microfabrication-compatible $\mathrm{PH}-$ SEC SWS has an excellent potential to realize BWOs which can operate at millimetre wave frequencies. 


\section{Table of Contents}

Statement of Originality

Supervisor Declaration Statement

Authorship Attribution Statement

Acknowledgement........................................................................................................i

Abstract ................................................................................................................................ii

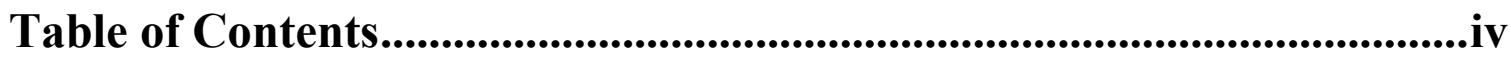

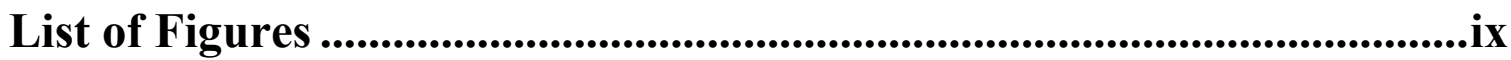

List of Tables............................................................................................................... xviii

List of Abbreviations and Symbols ........................................................xix

Chapter 1 Introduction ...........................................................................1

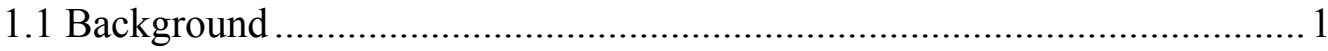

1.1.1 Vacuum Electron Devices (VEDs) ............................................... 1

1.1.2 Backward-Wave Oscillator (BWO) ........................................... 2

1.1.3 Slow-Wave Structures (SWSs) ................................................... 4

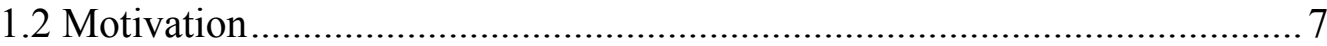

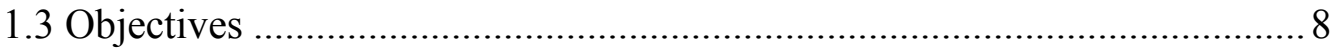

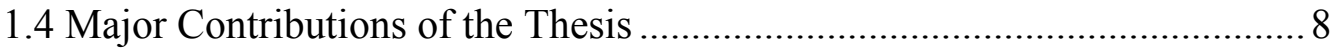

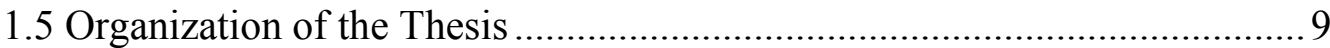

Chapter 2 Literature Review..............................................................................12

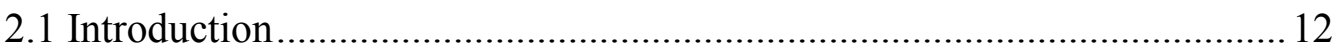

2.2 Basic Parameters of Slow-Wave Structure (SWS) .................................. 12

2.2.1 Dispersion Diagram ....................................................................... 13

2.2.2 Interaction Impedance ................................................................. 14

2.2.3 Estimation Methods ................................................................... 16

2.3 Principle of Operation of TWTs ............................................................. 17 
2.3.1 Travelling-Wave Tube Amplifier (TWTA)

2.3.2 Backward-Wave Amplifier (BWA)

2.3.3 Backward-Wave Oscillator (BWO) ............................................ 20

2.4 Backward-Wave Oscillators ................................................................ 20

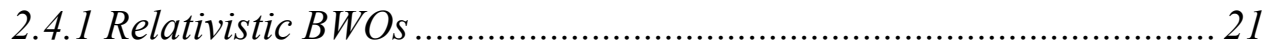

2.4.2 Low Voltage (Non-Relativistic) BWOs ......................................... 22

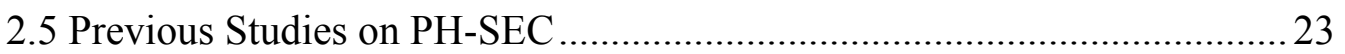

2.5.1 Dispersion Control .................................................................... 23

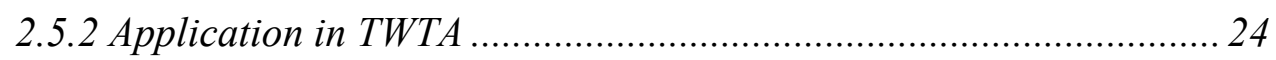

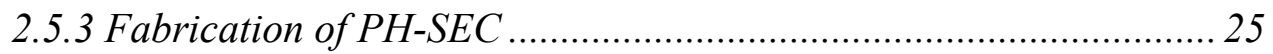

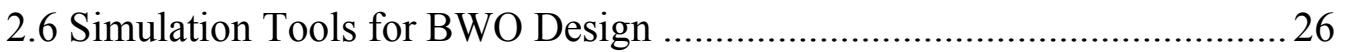

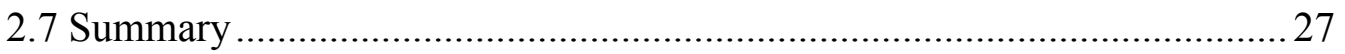

\section{Chapter 3 INTERACTION IMPEDANCE OF NON-FUNDAMENTAL SPACE HARMONICS........................................................................................28}

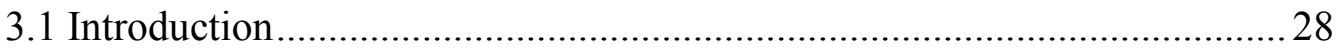

3.2 Methods to Find the Interaction Impedance of the Fundamental Space Harmonic

3.2.1 Analytical Modelling ..................................................................... 29

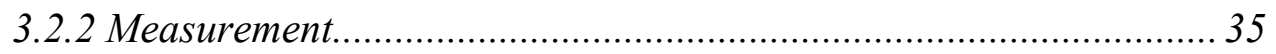

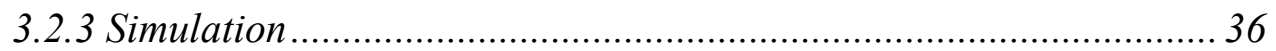

3.3 Comparison Between Tape-Helix Analysis and Simulation ......................37

3.3.1 Magnitude of longitudinal electric field for different space harmonics from simulation

3.3.2 Magnitude of longitudinal electric field for different space harmonics from analysis

3.3.3 Comparison of Results.....

3.3.4 Limitations of Tape-Helix Analysis

3.4 Interaction Impedance of Space Harmonics using Fourier Decomposition 54 


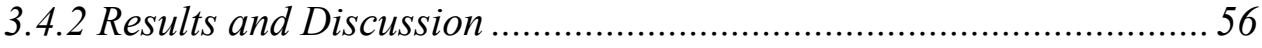

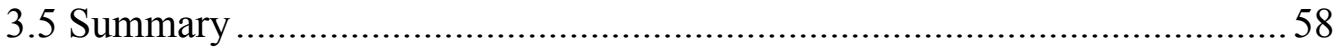

Chapter 4 Tape-Helix Analysis of PH-SEC .....................................................60

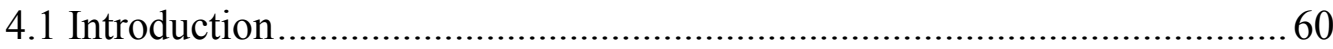

4.2 Tape-Helix Analysis of PH-SEC in a Homogeneous Medium................... 62

4.2.1 Tape-Helix Analysis of Infinitely Wide Planar Helix ..................... 62

4.2.2 Effective Dielectric Constant (EDC) Method ................................6 67

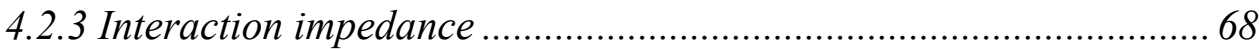

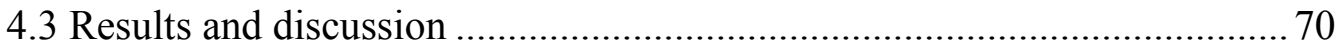

4.3.1 Experimental Validation of the Analysis ..................................... 73

4.3.2 Comparison with Tape-Helix Analysis of Rectangular Helix [86]... 79

4.3.3 Comparison with Tape-Helix Analysis in [101] ............................8 80

4.4 Analysis of PH-SEC in a Metal Enclosure …........................................... 82

4.4.1 Analysis of Infinitely Wide Planar Helix Shielded by Infinitely Long Metal Plates ................................................................................... 83

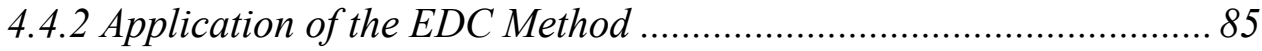

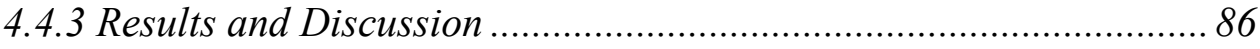

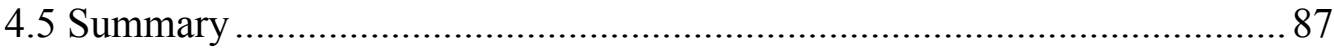

Chapter 5 A W-Band Backward-Wave Oscillator.....................................89

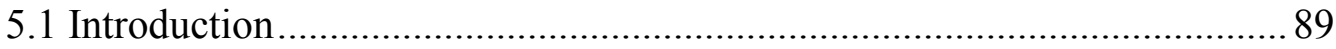

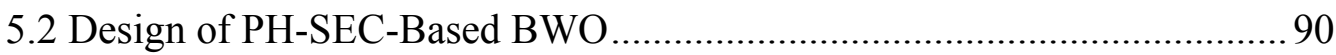

5.2.1 Configuration of the PH-SEC SWS............................................... 90

5.2.2 Dispersion Characteristics ................................................................ 91

5.2.3 Sensitivity to Geometrical Parameters .......................................... 92

5.2.4 Transmission and Reflection Characteristics ................................ 94

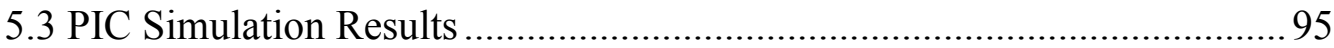


5.4 Fabrication of PH-SEC 100

5.4.1 Description of the Fabricated PH-SEC. 101

5.4.2 Measured Results 102

5.5 Summary 103

Chapter 6 Two-Beam Oscillator-Amplifier 105

6.1 Introduction 105

6.2 Concept of the Proposed Technique 107

6.3 Design of BWO and Oscillator-Amplifier Using Circular Helix 109

6.3.1 Properties of the SWS 109

6.3.2 Transmission and Reflection Characteristics. 111

6.3.3 Beam Configurations. 111

6.4 Comparison of PIC Simulation Results 112

6.4.1 $1^{\text {st }}$ Beam Configuration. 113

6.4.2 $2^{\text {nd }}$ Beam Configuration. 115

6.4.3 $3^{\text {rd }}$ Beam Configuration 118

6.5 Design of BWO and Oscillator-Amplifier Using PH-SEC 119

6.5.1 Design of PH-SEC 120

6.5.2 S-parameters of the BWO and oscillator-amplifier. 122

6.5.3 Beam Configuration 122

6.6 PIC Simulation Results 123

6.7 Summary 126

Chapter 7 Conclusion and Future Work 128

7.1 Conclusion 128

7.2 Recommendations for Further Research 130

Author's Publications. 133

Bibliography 134 
Appendix A: Interaction Impedance ..............................................................142

Appendix B: Calculation of Fourier coefficients .....................................143

Appendix C: Field Expressions for Transverse Fields ..............................144

Appendix D: MATLAB programme for finding the discrete Fourier transform of BWO output signal .....................................................................145 


\section{List of Figures}

Figure 1.1: Simplified schematic diagram of a backward-wave oscillator (BWO). .2

Figure 1.2: One period of circular helix SWS.

Figure 1.3: Perspective view of planar helix slow-wave structure with straight-edge connections (PH-SEC).

Figure 2.1: Dispersion diagram of a periodic SWS.

Figure 2.2: Schematic diagram of a travelling-wave tube.

Figure 2.3: Axial electric field for velocity modulation. 18

Figure 2.4: Perspective view of one period of the microfabrication-compatible $\mathrm{PH}$ SEC [32].

Figure 2.5: Perspective view of one period of the connected pair of PH-SECs [76].

Figure 3.1: (a) circular helix; (b) Sheath-helix model; (c) Side view of the sheathhelix model.

Figure 3.2: Circular tape-helix. 32

Figure 3.3: Circular tape-helix with dimensions.

Figure 3.4:.3D model of the tape-helix in Computer Simulation Technology (CST) eigenmode solver.

Figure 3.5: Real part of $E z r=0, \phi=0, z$ for $8.32 \mathrm{GHz}$. 40

Figure 3.6: Imaginary part of $E z r=0, \phi=0, z$ for $8.32 \mathrm{GHz}$. 40

Figure 3.7: Real part of $E z r=0, \phi=0, z$ for $8.32 \mathrm{GHz}$. .41

Figure 3.8: Imaginary part of $E z r=0, \phi=0, z$ for $8.32 \mathrm{GHz}$.

Figure 3.9: Variation of the Fourier coefficient for the fundamental space harmonic with number of mesh cells for $r=0, \phi=0$ and frequency of $8.32 \mathrm{GHz}$. 42

Figure 3.10: Variation of the Fourier coefficient for the $\mathrm{n}=-1$ space harmonic with number of mesh cells for $r=0, \phi=0$ and frequency of $8.32 \mathrm{GHz}$. 
Figure 3.11: Variation of the Fourier coefficient for the $n=+1$ space harmonic with number of mesh cells for $r=0, \phi=0$ and frequency of $8.32 \mathrm{GHz}$. 42

Figure 3.12: Variation of the Fourier coefficient for the $n=-2$ space harmonic with number of mesh cells for $r=0, \phi=0$ and frequency of $8.32 \mathrm{GHz}$

Figure 3.13: Variation of the Fourier coefficient for the $n=+2$ space harmonic with number of mesh cells for $r=0, \phi=0$ and frequency of $8.32 \mathrm{GHz}$.

Figure 3.14: Variation of the Fourier coefficient for the fundamental space harmonic with number of mesh data points for $r=0, \phi=0$ and frequency of $8.32 \mathrm{GHz}$....44 Figure 3.15: Variation of the Fourier coefficient for the $n=-1$ space harmonic with number of mesh data points for $r=0, \phi=0$ and frequency of $8.32 \mathrm{GHz}$. .44

Figure 3.16: Variation of the Fourier coefficient for the $n=+1$ space harmonic with number of mesh data points for $r=0, \phi=0$ and frequency of $8.32 \mathrm{GHz}$

Figure 3.17: Variation of magnitude of Ez vs. frequency for the fundamental space harmonic at axis of the helix with different separations of the PEC boundary from the circular tape-helix.

Figure 3.18: Variation of magnitude of Ez vs. radial distance from the axis of the SWS for the fundamental space harmonic from the circular tape-helix 45

Figure 3.19: Comparison of dispersion characteristics for the circular helix 47

Figure 3.20: Comparison of normalized values of the magnitude of $E z$ for the fundamental space harmonic at two different off-axis positions; $a$ is the inner radius of the helix

Figure 3.21: Comparison of normalized values of the magnitude of $E z$ for the $n=-1$ space harmonic at two different off-axis positions; $a$ is the inner radius of the helix. 48

Figure 3.22: Comparison of normalized values of magnitude of $E z$ from simulations for the fundamental and $n=-1$ space harmonic at on-axis and off-axis positions. ..49 Figure 3.23: Comparison of normalized values of magnitude of $E z$ from simulations for the first five space harmonics at on-axis position. 
Figure 3.24: The side view of the circular helix with coordinate system. The $\mathrm{z}$ axis is going into the plane of the figure.

Figure 3.25: The perspective view of the circular tape helix with two lines drawn on the inner surface of the tape, corresponding to $\phi=90^{\circ}$ (line 1) and $\phi=180^{\circ}$ (line 2). The Cartesian coordinate system is also shown; the origin of the coordinate system is at $\mathrm{z}=0$.

Figure 3.26: (a) Magnitude and (b) phase of the total current density along one period of tape helix for three different frequencies from simulation. This figure is for line-1. The tape extends from $\mathrm{z}=-0.04 \mathrm{~mm}$ to $\mathrm{z}=+0.04 \mathrm{~mm}$. The values of phase from analysis for these three frequencies are also shown in (b).

Figure 3.27: Normalized values of (a) magnitude and (b) phase of the total current density along one period of tape helix for three different frequencies from simulation. This figure is for line- 1 . The tape extends from $z=-0.04 \mathrm{~mm}$ to $z=+0.04 \mathrm{~mm}$. The normalized values of the expected values of phase from analysis for these three frequencies are also shown in (b).

Figure 3.28: (a) Magnitude and (b) phase of the total current density along one period of tape helix for three different frequencies from simulation. This figure is for line-2. The tape extends from $\mathrm{z}=+0.16 \mathrm{~mm}$ to $\mathrm{z}=+0.24 \mathrm{~mm}$. The expected values of phase from analysis for these three frequencies are also shown in (b). .52

Figure 3.29: (a) Normalized values of (a) magnitude and (b) phase of the total current density along one period of tape helix for three different frequencies from simulation. This figure is for line -2 . The tape extends from $z=+0.16 \mathrm{~mm}$ to $\mathrm{z}=+0.24 \mathrm{~mm}$. The normalized values of the expected values of phase from analysis for these three frequencies are also shown in (b). 53

Figure 3.30: (a) Normalized values of (a) magnitude, and (b) phase of the total current density along the width of the tape for three different frequencies. This figure is for line -1 . The tape extends from $\mathrm{z}=-0.04 \mathrm{~mm}$ to $\mathrm{z}=+0.04 \mathrm{~mm}$. So, $\mathrm{w}=0$ corresponds to $\mathrm{z}=-0.04 \mathrm{~mm}$ and $\mathrm{w}$ corresponds to $\mathrm{z}=+0.04 \mathrm{~mm}$.

Figure 3.31: Simulation values of interaction impedance of the fundamental space harmonic in the circular helix at different positions. 56 
Figure 3.32: Simulation values of interaction impedance of the $n=-1$ space harmonic in the circular helix at different positions.

Figure 3.33: Front view of the circular helix based practical slow-wave structure with dimensions.

Figure 3.34: Simulation values of interaction impedance of the fundamental space harmonic in the practical structure at different positions.

Figure 3.35: Simulation values of interaction impedance of the $n=-1$ space harmonic in the practical structure at different positions. .58

Figure 4.1: Perspective views of a) PH-SEC, and b) rectangular helix. .60

Figure 4.2: (a) Plan view and (b) cross-sectional view of infinitely wide planar helix.

Figure 4.3: Model for applying EDC method: (a) $x$-dependent profile; (b) $y$-dependent profile.

Figure 4.4: Comparison of simulated and analytical results for a PH-SEC for three different pitch angles $\left(\psi=2.5^{\circ}, 5^{\circ}, 7.5^{\circ}\right)$ with $a / b=0.25, a=0.48 \mathrm{~mm}$, and $w / L=$ 0.2. (a) Normalized phase velocity, (b) Interaction impedance

Figure 4.5: Comparison of simulated and analytical results for a PH-SEC for three different pitch angles $\left(\psi=2.5^{\circ}, 5^{\circ}, 7.5^{\circ}\right)$ with $a / b=1, a=1.2 \mathrm{~mm}$, and $w / L=0.2$. (a) Normalized phase velocity, (b) Interaction impedance. .70

Figure 4.6: Comparison of simulated and analytical results for a $\mathrm{PH}-\mathrm{SEC}$ for three different pitch angles $\left(\psi=2.5^{\circ}, 5^{\circ}, 7.5^{\circ}\right)$ with $a / b=4, a=1.92 \mathrm{~mm}$, and $w / L=0.2$. (a) Normalized phase velocity, (b) Interaction impedance. .71

Figure 4.7: Comparison of simulated and analytical results at W-band for a PH-SEC for three different pitch angles $\left(\psi=2.5^{\circ}, 5^{\circ}, 7.5^{\circ}\right)$ with $a / b=1, a=0.15 \mathrm{~mm}$, and $w / L$ $=0.2$. (a) Normalized phase velocity, (b) Interaction impedance.

Figure 4.8: Dispersion diagram of a PH-SEC with $a / b=1, a=1.2 \mathrm{~mm}, w / L=0.2$, and $a / L=1$. .73

Figure 4.9: Fabricated prototype of the SWS with 30 periods. (a) The SWS before assembling top and bottom dielectric sheets; (b) the final SWS. .73 
Figure 4.10: Comparison of the S11 of the SWS with number of mesh cells..........75

Figure 4.11: Comparison of the S21 of the SWS with number of mesh cells. 75

Figure 4.12: Comparison of measured and simulated S-parameters of SWS with 30 periods. 76

Figure 4.13: Perspective view of the SWS with 30 periods used in Transient mode solver. 77

Figure 4.14: Zoomed version of S11 of the SWS 77

Figure 4.15: Input and output signals from Transient solver. The red curve represents the input signal applied at port-1, the blue curve represents the output signal obtained at port-2, and the green curve represents the reflected signal obtained at port-1.... 77

Figure 4.16: A zoomed version of the reflected signal obtained at port-1 78

Figure 4.17: Comparison of normalized phase velocity of the PH-SEC. 79

Figure 4.18: Comparison of normalized phase velocity for a rectangular tape helix with $b / a=10, L / a=2, w / L=0.2$ and $a=0.232 \mathrm{~mm}$ 79

Figure 4.19: Comparison of normalized phase velocity for a rectangular tape-helix with $b / a=1, L / a=1, w / L=0.1$ and $a=15.85 \mathrm{~mm}$ 80

Figure 4.20: Comparison of normalized phase velocity of a PH-SEC with $b / a=4$, $w / L=0.2$, and $a=0.75 \mathrm{~mm}$.

Figure 4.21: Comparison of normalized phase velocity of a PH-SEC with $b / a=1$, $w / L=0.2$, and $a=1.5 \mathrm{~mm}$

Figure 4.22: PH-SEC inside a metal enclosure. 83

Figure 4.23: (a) Plan view (metal plates are hidden) and (b) cross-sectional view of the infinitely wide planar helix shielded by a pair of infinitely wide metal plates. .83

Figure 4.24: Comparison of simulation and analytical results for the shielded PHSEC for three different pitch angles $\left(\psi=2.5^{\circ}, 5^{\circ}, 7.5^{\circ}\right)$ with $a / b=1, a=1.2 \mathrm{~mm}, d$ $=2 \mathrm{~mm}$ and $w / L=0.2$. (a) Normalized phase velocity; (b) Interaction impedance 85 
Figure 4.25: Comparison of simulated and analytical results for a PH-SEC inside a metal enclosure for two different size for metal enclosure $(d=0.2 \mathrm{~mm}, 2 \mathrm{~mm})$ with $a / b=1, a=1.2 \mathrm{~mm}$, and $w / L=0.2$. (a) Normalized phase velocity; (b) Interaction impedance. .86

Figure 5.1: Cross-sectional view of the PH-SEC SWS with dimensions (all dimensions are in $\mu \mathrm{m})$.

Figure 5.2: Dispersion characteristics of the SWS with beam line corresponding to $8.5 \mathrm{KV}$ beam voltage.

Figure 5.3: On-axis interaction impedance of $n=-1$ space harmonic of the SWS...91

Figure 5.4: Dispersion characteristics with different values of (a) $h 1$, (b) $h 2$, and (c) gl. Zoomed in parts of the curves are also shown

Figure 5.5: Variation of the interaction impedance with (a) $h 1$, (b) $h 2$, and (c) $g 1$. Zoomed-in parts of the figures are also given.

Figure 5.6: The PH-SEC SWS with 4 periods and stripline feeds. The metal enclosure and dielectric supports are not shown. (a) 3-D view, and (b) plan view with main dimensions (all dimensions are in $\mu \mathrm{m}$ ).

Figure 5.7: S-Parameters of the PH-SEC SWS for BWO.

Figure 5.8: The output signal versus time of the BWO with $8.5 \mathrm{KV}$ beam voltage; zoomed version of signal is in the inset.

Figure 5.9: Discrete Fourier transform of BWO output at time window between 200 ns to $250 \mathrm{~ns}$

Figure 5.10: Discrete Fourier transform of BWO output at time window between 100 ns to $250 \mathrm{~ns}$

Figure 5.11: Phase space energy of the electrons vs. the longitudinal position at 30 ns.

Figure 5.12: Variation of the output power and frequency with beam voltage for three different beam currents.

Figure 5.13: Peak DC-to-RF conversion efficiency of the BWO versus frequency for three different beam currents. 100 
Figure 5.14: Structure and dimensions of the fabricated PH-SEC SWS (all dimensions are in millimetre).

Figure 5.15: Prototype assembly of the SWS with 30 periods. (a) Top view. (b) Bottom view. (c) Side view. (d) SWS inside the metal enclosure (without the top cover of the metal enclosure).

Figure 5.16: Comparison of measured and simulated S-parameters.

Figure 5.17: Measured and simulated normalized phase velocity of the SWS.

Figure 6.1: Schematic diagram of the (a) traveling-wave tube (TWT); (b) proposed vacuum electronic two-beam oscillator-amplifier. 108

Figure 6.2: Cross-section and perspective views of the circular helix SWS used for designing BWO and oscillator-amplifier. 109

Figure 6.3: Dispersion characteristics of the SWS with three beam lines. 110

Figure 6.4: Interaction impedance of the fundamental $\left(\mathrm{K}_{0}\right)$ and $n=-1\left(\mathrm{~K}_{-1}\right)$ space harmonic of the circular helix SWS at four different radial positions.

Figure 6.5: S-parameters of the SWS used as BWO and as oscillator-amplifier... 111 Figure 6.6: Three configurations of the beams considered for the proposed oscillatoramplifier.

Figure 6.7: Output signal from both oscillator-amplifier and conventional BWO. the oscillator-amplifier uses a $4 \mathrm{KV}$ annular beam and a $17.2 \mathrm{KV}$ solid beam, whereas the conventional BWO uses the $4 \mathrm{KV}$ annular beam ( $1^{\text {st }}$ beam configuration $) . . . .114$ Figure 6.8: Discrete Fourier transform of the output signal from the oscillatoramplifier at two different beam voltages for the annular beam ( $1^{\text {st }}$ beam configuration). 114

Figure 6.9: Output signal from the oscillator-amplifier with $4 \mathrm{KV}$ and $17.2 \mathrm{KV}$ solid beams, and the corresponding output signal from the conventional BWO with $4 \mathrm{KV}$ solid beam ( $2^{\text {nd }}$ beam configuration).

Figure 6.10: Discrete Fourier transform of the output signal from the oscillatoramplifier at two different beam voltages ( $2^{\text {nd }}$ beam configuration). 
Figure 6.11: Discrete Fourier transform of the output signal from the oscillatoramplifier with $4 \mathrm{KV}$ and $17.2 \mathrm{KV}$ solid beams for three different time intervals.. 116 Figure 6.12: Comparison of efficiency of the oscillator-amplifier and the conventional BWO.

Figure 6.13:Variation of frequency and power of the output signal with beam voltage for backward-wave interaction in the oscillator-amplifier.

Figure 6.14:Output signal from the oscillator-amplifier and the conventional BWO. The oscillator-amplifier has a $4 \mathrm{KV}$ solid beam outside the SWS and a $17.2 \mathrm{KV}$ solid beam inside the SWS ( $3^{\text {rd }}$ beam configuration) whereas the conventional BWO has a $4 \mathrm{KV}$ solid beam outside the SWS.

Figure 6.15: Discrete Fourier transform of the output signal from the oscillatoramplifier at two different beam voltages for the beam outside the SWS ( $3^{\text {rd }}$ beam configuration).

Figure 6.16:Cross-sectional and the perspective view of the PH-SEC. 120

Figure 6.17: Dispersion characteristics of the SWS; also shown are three different beam lines.

Figure 6.18: Interaction impedance of the fundamental $\left(\mathrm{K}_{0}\right)$ and $n=-1\left(\mathrm{~K}_{-1}\right)$ space harmonic of the PH-SEC at three different beam positions; $(\mathrm{x}, \mathrm{y})$ are the coordinates of the beam position.

Figure 6.19: S-parameters of the PH-SEC SWS used as BWO and as oscillatoramplifier.

Figure 6.20: Position of E-beams for the BWO and the oscillator-amplifier. 123

Figure 6.21: Output signal from the oscillator-amplifier with $5 \mathrm{KV}$ and $15.75 \mathrm{KV}$ solid beams, and the output signal from the BWO with $5 \mathrm{KV}$ solid beam.

Figure 6.22: Discrete Fourier transform of the output signal from the oscillatoramplifier at two different beam voltages for backward-wave interaction. 124

Figure 6.23: Comparison of efficiency of the oscillator-amplifier and the conventional BWO. 124 
Figure 6.24: Variation of frequency and power of the output signal with beam voltage for the backward-wave interaction in the oscillator-amplifier. 125 


\section{List of Tables}

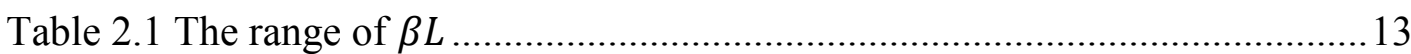

Table 5.1 Strength of various peaks in the Spectrum ..............................................98

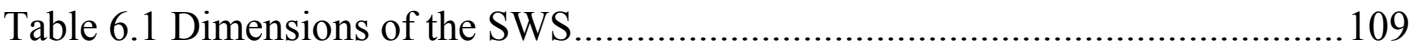

Table 6.2 Comparison of output performance of the conventional BWO and the two-

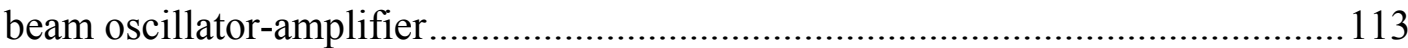

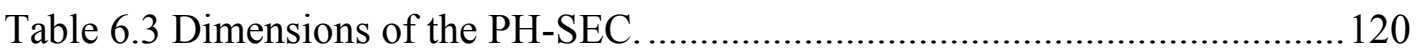




\section{List of Abbreviations and Symbols}

\section{Abbreviations}

$3 \mathrm{D}$

Three dimensional

BCB bisbenzocyclobutene

BWA Backward-wave amplifier

BWO Backward-wave oscillator

CST Computer Simulation Technology

CW Continuous wave

DRIE Deep reactive ion etching

ECM Electronic countermeasures

EDC Effective dielectric constant method

EDM Electrical discharge machining

EM Electromagnetic

FDTD Finite difference time domain

FEM Finite element method

FIT Finite integration technique

HFSS High Frequency Structure Simulator

LIGA Lithographe, Galvonoformung, und Abfornung and translates as lithography, electroplating, and micro-moulding

MWS Microwave Studio

PH Planar helix

PH-SEC Planar helix slow-wave structure with straight-edge connections

PIC Particle-in-cell

PPM Periodic-permanent-magnet 


\begin{tabular}{ll}
\hline PTH & Plated through holes \\
SWS & Slow-wave structure \\
TE & Transverse electric \\
TLM & Transmission-line-matrix \\
TM & Transverse magnetic \\
TWT & Travelling-wave tube \\
TWTA & Travelling-wave tube amplifier \\
VED & Vacuum electron device \\
DFT & Discrete Fourier transform
\end{tabular}

\section{Symbols}

c Speed of light in free space

$E_{z} \quad$ Axial electric field

$\left|E_{z, n}(r, \phi)\right| \quad$ Magnitude of the longitudinal electric field for the $n^{\text {th }}$ space harmonic at the position $(r, \phi)$

$\left|E_{z}(r, \phi)\right| \quad$ Magnitude of the total longitudinal electric field at the position $(r, \phi)$

$f \quad$ Frequency

$H_{z} \quad$ Axial magnetic field

$I_{0} \quad$ Zeroth-order modified Bessel function of the first kind

$I_{1} \quad$ First-order modified Bessel function of the first kind

$I_{m} \quad m^{\text {th }}$ order modified Bessel functions of the first kind

$I_{n}^{\prime} \quad$ Derivative of the $n^{\text {th }}$ order modified Bessel function of first kind

$J \quad$ The total surface current parallel to the winding

$J_{n} \quad n^{\text {th }}$ space harmonic component of the surface current parallel to the winding 


\begin{tabular}{|c|c|}
\hline$\overline{J_{z n}}$ & $\begin{array}{l}n^{\text {th }} \text { space harmonic component of the axial component of surface current } \\
\text { density }\end{array}$ \\
\hline$J_{\phi n}$ & $\begin{array}{l}n^{\text {th }} \text { space harmonic component of the azimuthal component of surface } \\
\text { current density }\end{array}$ \\
\hline$k$ & Wavenumber \\
\hline$k_{0}$ & Free space wavenumber \\
\hline$K_{0}$ & Interaction impedance of fundamental space harmonic \\
\hline$K_{0}$ & Zeroth-order modified Bessel function of the second kind* \\
\hline$K_{-1}$ & Interaction impedance of $n=-1$ space harmonic \\
\hline$K_{1}$ & First-order modified Bessel function of the second kind \\
\hline$K_{m}$ & $m^{\text {th }}$ order modified Bessel functions of the second kind \\
\hline$K_{n}$ & Interaction impedance of $n^{\text {th }}$ space harmonic \\
\hline$K_{n}(r, \phi)$ & Interaction impedance of $n^{\text {th }}$ space harmonic at the position $(r, \phi)$ \\
\hline$K_{n}^{\prime}$ & Derivative of the $n^{\text {th }}$ order modified Bessel function of second kind \\
\hline$L$ & Pitch length \\
\hline$n$ & Number of space harmonic \\
\hline$P$ & Total RF power propagating through the SWS \\
\hline$p_{y}$ & Periodic spacing between strips in planar helix along $y$-and direction \\
\hline$p_{z}$ & Periodic spacing between strips in planar helix along $z$-and direction \\
\hline$r, \phi, z$ & Circular cylindrical coordinates \\
\hline S11 & Reflection coefficient \\
\hline $\mathrm{S} 21$ & Transmission coefficient \\
\hline$v_{g}$ & Group velocity \\
\hline$v_{p}$ & Phase velocity \\
\hline$w$ & Actual width of the strip in planar helix \\
\hline$w_{y}$ & Width of the strip in planar helix along $y$ - direction \\
\hline
\end{tabular}




\begin{tabular}{|c|c|}
\hline$w_{z}$ & Width of the strip in planar helix along $z$ - direction \\
\hline$x, y, z$ & Cartesian coordinates \\
\hline$\beta$ & Phase constant \\
\hline$\beta_{0}$ & Phase constant of the fundamental space harmonic \\
\hline$\beta_{n}$ & Phase constant of $n^{\text {th }}$ fundamental space harmonic \\
\hline$\gamma_{\mathrm{n}}$ & Wave number for the $n^{\text {th }}$ space harmonic \\
\hline$\varepsilon$ & Permittivity of a medium \\
\hline$\varepsilon_{\mathrm{r}}$ & Relative Permittivity of a medium \\
\hline$\varepsilon_{0}$ & Permittivity of free space \\
\hline$\varepsilon_{e f f}$ & Effective dielectric constant \\
\hline$\varepsilon_{\mathrm{r}}$ & Relative permittivity of medium \\
\hline$\mu$ & Permeability of a medium \\
\hline$\mu_{0}$ & Permeability of free space \\
\hline$\mu_{r}$ & Relative permeability of a medium \\
\hline$\eta$ & DC-to-RF conversion efficiency \\
\hline$\sigma$ & Conductivity \\
\hline$\Psi$ & Pitch angle \\
\hline Yeff & Effective pitch angle for PH-SEC \\
\hline$\omega$ & Angular frequency \\
\hline
\end{tabular}

*This symbol is also used for the interaction impedance of fundamental space harmonic. But the context in the text makes it clear which parameter/function is being referred to. 


\section{Chapter 1 Introduction}

\subsection{Background}

\subsubsection{Vacuum Electron Devices (VEDs)}

Importance of microwave $(0.3-30 \mathrm{GHz})$ and millimetre-wave $(30-300 \mathrm{GHz})$ frequencies is increasing due to their wide range of applications in cellular communications, satellite communications, deep space communications, radar systems, spectroscopy, electronic countermeasures (ECM), material processing, high-power microwave weapons, etc. [1]. Solid-state electron devices and vacuum electron devices (VEDs) are the commonly used amplifiers and oscillators for this frequency. Both types of devices have a common operating principle of converting the kinetic energy of an electron current into the energy of an electromagnetic wave.

Solid-state high frequency oscillators are attractive due to their small size and ease of fabrication. But the speed of charge carriers in these devices is limited by the solid semiconducting medium. Besides, solid-state devices require bulky heat sinks to conduct away the heat produced by collisions of charge carriers. Moreover, the temperature rise in solid-state devices causes significant carrier mobility reduction and chemical degradation of semiconducting channel. Another disadvantage of solid-state devices is electrical breakdown at high voltages which prevents their high-power operation.

In contrast, VEDs are able to operate at higher temperature and generate much higher output power than solid-state devices. The vacuum environment in VEDs avoids the limitation in the speed due to collision of charge carriers. Thus, VEDs can avoid the abovementioned problems in solid-state devices. As a result, VEDs are still preferred for high frequency and high-power applications. Traveling wave tubes (TWTs), klystrons, magnetrons, gyrotrons etc., are some of the important VEDs [2], [3]. VEDs can work in the frequency range from below $100 \mathrm{MHz}$ to Terahertz and some of these are capable of generating an output power up to several megawatts.

TWTs are unique among VEDs due to their wide bandwidth and moderately high efficiency. Besides, TWTs have much longer operating life ( $>15$ years), and higher reliability than solid state devices. Backward-wave oscillator (BWO) and travelling-wave tube amplifier (TWTA) are the two important types of devices that come under this 
category. BWO is the most reliable voltage tuneable oscillator with a high purity frequency spectrum, whereas TWTA is a wide band amplifier with high efficiency and moderately high output power.

\subsubsection{Backward-Wave Oscillator (BWO)}

BWO is one of the most established VED for generating electromagnetic waves with frequencies up to THz. This device is an electronically tuneable oscillator, i.e., the frequency of the generated signal in this device can be tuned by varying the beam voltage. Pure frequency spectrum and wide tuneable bandwidth $(\sim 50 \%)$ are the advantages of this device. The frequency of the output signal in a BWO is stable and relatively independent of load impedance [2]. BWO has a wide range of applications since it can provide fast electronically controlled frequency sweep. BWOs are commonly used as local oscillators in fast-tuning receivers for remote sensing and low-background astronomy observations [2]. Terahertz imaging and spectroscopy are the other important applications of the BWO [4], [5]. Recently a $0.346-\mathrm{THz}$ BWO has been fabricated for plasma collective scattering diagnostics [6]. BWO is very useful for high-frequency phase-sensitive measurements like interferometry due to its electronic tuneability and high phase stability.

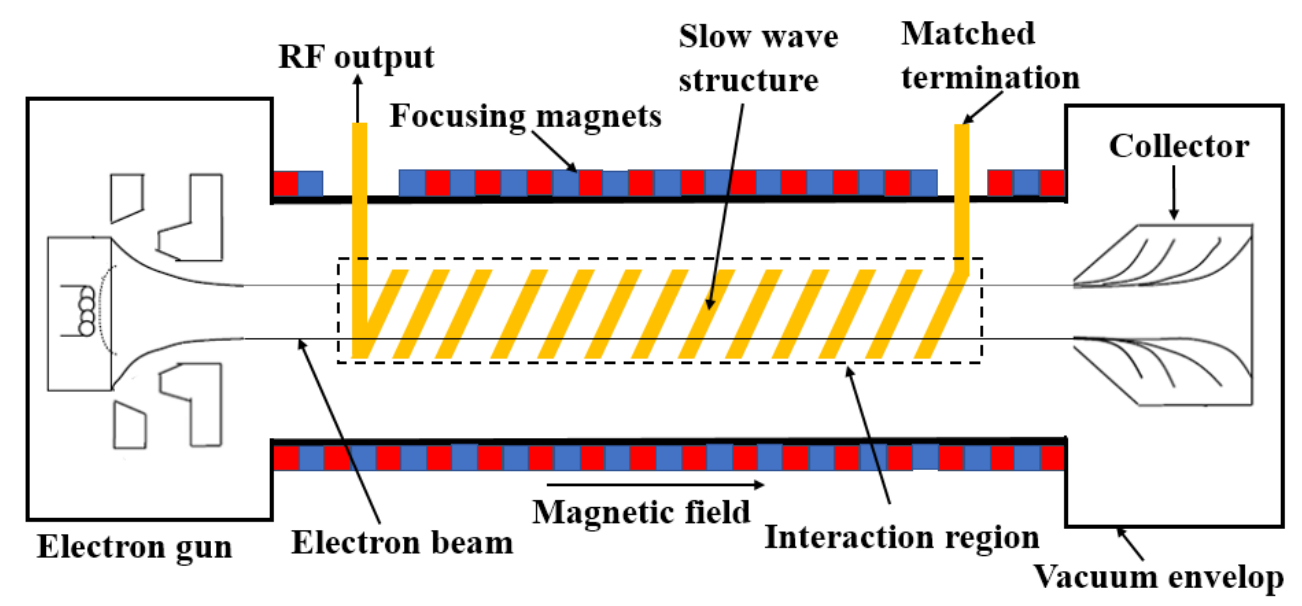

Figure 1.1: Simplified schematic diagram of a backward-wave oscillator (BWO).

A simplified schematic diagram of the BWO is shown in Fig. 1.1. As shown in the figure, an electron gun, an array of focusing magnets, a slow-wave structure (SWS), an RF output port, and a collector are the important parts of a BWO. The entire device from the electron gun to the collector is enclosed in a vacuum envelope. A cathode is used in the electron gun to emit the electrons. The electron gun also contains focusing and accelerating electrodes for converting the emitted electrons into an electron beam with a specific beam 
velocity. The collector is used at the end of the BWO to collect and recover the energy from the spent beam. Solenoid or periodic-permanent-magnet (PPM) can be used as focusing magnet for generating a magnetic field along the direction of the flow of electrons. The focusing magnetic field is required for keeping the shape of the electron beam by counteracting the radial space charge forces between the electrons.

The SWS is a very important component in a BWO since it determines the electromagnetic properties of the device. SWS helps to slow down the phase velocity of the electromagnetic waves travelling through it and synchronizes it with the speed of the electron beam. The process of synchronizing the beam velocity of the electron beam with the phase velocity of the electromagnetic wave is called "velocity synchronization". Commonly, periodic structures like circular helix are used as the SWS in BWOs. The periodicity of SWS leads to the phenomenon of space harmonics. Space harmonics are electromagnetic waves with the same frequency but different phase velocities. In general, an infinite number of space harmonics coexist in a SWS [7]. The fundamental $(n=0)$ space harmonic of common SWSs is a forward-wave, i.e., the phase velocity and group velocity are in the same direction, whereas the $n=-1$ space harmonic is a backward-wave with oppositely directed phase velocity and group velocity. The phase velocity is the velocity with which the phase of an EM wave propagates, whereas the group velocity is the velocity of the energy of an EM wave.

The first step for successful oscillation in a BWO is the velocity synchronization between the electron beam and the $n=-1$ space harmonic. Second step is to increase the beam current of electron beam above a specific value called as "starting current". Once these two conditions are satisfied, the electron bunches are generated according to the frequency at which the velocity synchronization occurs. Then the bunched electrons induce a travelling EM wave in the SWS, and the induced wave again improves the strength of electron bunches. This process continues and finally the output signal is taken out through the port near the electron gun. In a BWO, the second port, i.e., the port near the collector is match-terminated to avoid reflections.

Continuous interaction and energy transfer between the electron beam and EM wave is the basic operating principle of TWTs. In a BWO the phase of the generated EM wave propagates in the direction of the electron beam whereas the energy of the generated EM wave propagates in the opposite direction. The strength of electron bunches is stronger near 
the collector whereas the strength of the generated EM wave is stronger near the electron gun. Hence the efficiency of extracting energy from the electron bunces is moderate in BWOs since an efficient extraction requires a higher strength of the EM wave.

\subsubsection{Slow-Wave Structures (SWSs)}

SWS is the heart of a BWO since it helps in the velocity synchronization. The existence of space harmonics in the SWS is an outcome of its spatial periodicity. While in general an infinite number of backward-wave space harmonics are present in a periodic SWS, the $n=-1$ space harmonic has the maximum strength among them. Hence the $n=-1$ space harmonic is commonly used for realizing BWOs.

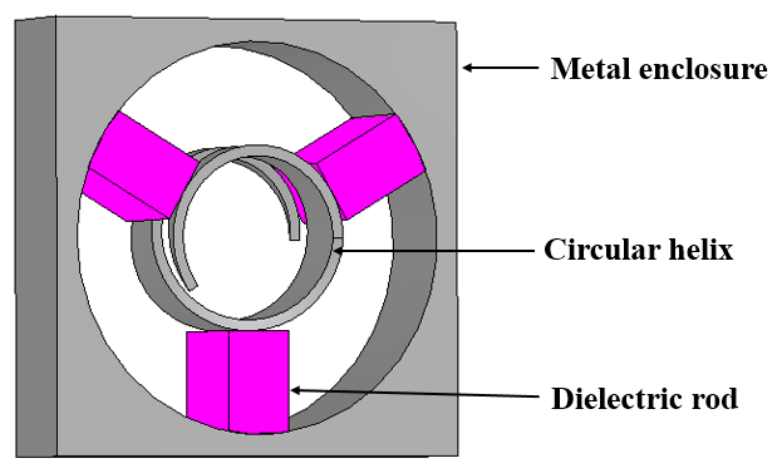

Figure 1.2: One period of circular helix SWS.

Ridge-waveguide SWS was used for one of the initial experiments on BWOs [8]. Johnson used a BWO with circular helix SWS for validating his theoretical studies on BWOs [9]. Circular helix is the most popular SWS for realizing TWTs due to its unbeatable bandwidth and strong beam-wave interaction. Recent research efforts are focusing on increasing the operating frequency of circular helix based BWOs [10]. As shown in Fig. 1.2, the circular helix is usually supported inside a metal enclosure using dielectric support rods. The limited area of cross-section of the dielectric rods limits the heat dissipation capacity of this SWS and ultimately limits the maximum operating power. Therefore SWSs such as corrugated waveguide, double corrugated waveguide, sinusoidally corrugated waveguide, and folded waveguide are used for high power applications [6] [10]-[15]. These SWSs are designed without dielectric parts for improved thermal conductivity. BWOs with these SWSs sacrifice bandwidth for achieving high power operation.

Nowadays researchers are also investigating SWSs with metamaterials and photonic crystals for improving the performance of BWOs. Metamaterial-based SWSs have been developed for achieving high output power and high efficiency [16]-[18]. Complimentary 
$\frac{\text { Chapter } 1}{\text { split ring resonators are periodically loaded inside a metallic waveguide to realize }}$ metamaterial-based SWS. The waveguide is operated below the cut-off frequency to provide negative permittivity whereas the complimentary split ring resonator provides negative permeability in the SWS. The space harmonics are the reason for negative group velocity in normal periodic SWS whereas simultaneous negative permittivity and negative permeability provide negative group velocity in metamaterial SWSs. Narrow bandwidth is the main drawback of such SWSs.

The concept of photonic crystals has been used for designing novel SWSs and also for improving the performance of existing SWSs [19]-[21]. A novel SWS for BWO has been designed by arranging metal rods in a particular pattern and creating some intentional defects in [19], [20]. Photonic crystals have been used as the side wall of the corrugated rectangular waveguide SWS. Side walls with photonic crystals can provide more spacing around the central corrugations and thus reduce the difficulty in vacuum pumping. Photonic crystals have also been used for designing couplers with minimum loss in BWOs [22].

\subsubsection{MICROFABRICATION OF SWSS}

The dimensions of SWS and beam tunnel in TWTs are inversely proportional to the frequency of operation. For instance, the radius of circular helix scales down from $2.4 \mathrm{~mm}$ to $0.1 \mathrm{~mm}$ when the operating frequency increases from $4 \mathrm{GHz}$ to $95 \mathrm{GHz}$. Reduced size of SWS leads to the requirement of size reduction of other components in the TWT. Fabrication of TWTs at higher frequencies is problematic due to the difficulty in achieving the required machining tolerances even in advanced computer controlled milling machines and lathes. Besides, accurate alignment of components is another challenge for realizing high frequency TWTs. Small misalignment in focusing magnet or SWS in a TWT can result in a poor beam transport and collision of electron beam with the SWS. Poor beam transport can result in partial or complete reduction in the performance of the device. Moreover, the collisions can lead to high temperature and complete destruction of the device. Accurate fabrication and alignment of circular helix TWTs using conventional methods is challenging as the frequency of operation extends beyond $100 \mathrm{GHz}$ [23].

Recent advances in microfabrication techniques have enabled accurate fabrication of various components in TWTs with extremely small dimensions. Microfabrication techniques have the capability to realize both metal and dielectric structures together with good precision. Moreover, different components in a TWT like electron gun, SWS, and 
collector can be fabricated together on the same substrate using microfabrication technique, which reduces the challenge in alignment of various components. In contrast to the conventional fabrication techniques, microfabrication techniques offer the potential of mass production and repeatable accuracy. LIGA (Lithographe, Galvonoformung, und Abfornung and translates as lithography, electroplating, and micro-moulding), deep reactive ion etching (DRIE), electrical discharge machining (EDM), electrical milling, and dicing, are the widely used microfabrication techniques for fabricating different components of TWTs [24]. Different SWSs such as the folded waveguide [25]-[26], meander-line [27], coupled cavity [28], circular helix [10], and planar helix slow-wave structure with straight-edge connections (PH-SEC) [29] have been developed using microfabrication techniques.

\subsubsection{PlanaR HeliX SlOW-WAVE STRUCTURE With STRAIGHT-EDGE CONNECTIONS (PH-SEC)}

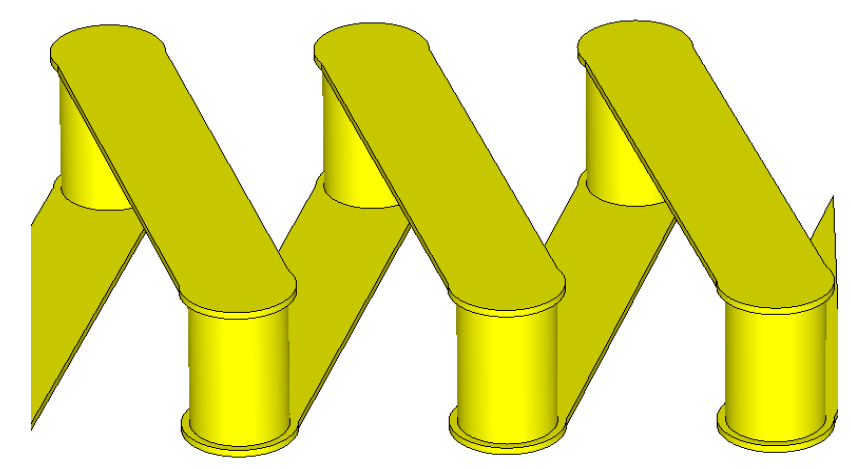

Figure 1.3: Perspective view of planar helix slow-wave structure with straight-edge connections (PH-SEC).

Even though circular helix is the most popular SWS due to its wide bandwidth and strong beam-wave interaction, it is not easily amenable to microfabrication techniques due to the circular geometry. In this context, PH-SEC, a planar counterpart of circular helix has been proposed and studied in recent years [29]-[33]. As shown in Fig. 1.3, the PH-SEC replaces the inclined edge connections between the top and bottom arrays of inclined strips of a rectangular helix by easily realizable straight-edge connections. PH-SEC sandwiched between two dielectric substrates has been fabricated using PCB fabrication to demonstrate the ease of fabrication of this structure [30]. Moreover, a W-band PH-SEC has been fabricated on a thick silicon substrate using microfabrication techniques [29]. The PH-SEC retains the wide bandwidth as well as the strong beam-wave interaction properties of the 
circular helix. Besides, the PH-SEC is compatible with the sheet electron beam which can offer many advantages such as decreased beam voltage, higher beam current capacity, and increased bandwidth for high-frequency TWTs [34].

A microfabrication-compatible TWTA to operate at Ka-band has been designed using PH-SEC [32]. The design shows the feasibility of microfabricating the PH-SEC with vacuum compatible materials. Furthermore, this SWS has very good thermal dissipation properties due to intimate contact between the helix and the supporting substrates. A sheet beam electron-optical system for W-band PH-SEC, which contains a beam forming electrode, anode, magnetic circuit and depressed collector has been reported in [33]. This design shows that all components except the magnetic circuit of a PH-SEC- based TWT at W-band can be microfabricated.

\subsection{Motivation}

Generation and amplification of millimetre wave and terahertz frequencies is important due to their wide range of applications. Vacuum electron devices like TWTs have superior performance than solid state devices at these frequencies. In this context, research on high frequency TWTs has grown in recent years. Developing microfabricationcompatible devices with good performance is the challenge in this area.

PH-SEC has been developed and studied in recent years for application in TWTAs. The studies have proved that this structure is microfabrication-compatible with good thermal dissipation properties. Moreover, the structure retains the wide bandwidth and strong beam-wave interaction properties of the circular helix.

Although many studies have been conducted for PH-SEC, there remain significant aspects that have not been investigated yet. First of all, the strength of different space harmonics in the PH-SEC has not been studied yet. This information is necessary for accurate evaluation of the interaction impedance for different space harmonics. Secondly, a field-theory based analysis for the accurate evaluation of dispersion characteristics of the PH-SEC has not been developed yet. The existing method does not consider the effect of space harmonics; hence its accuracy is not good enough. Thirdly, the existing studies of PH-SEC have focused on its application in TWTAs; a BWO with PH-SEC as the SWS has not been investigated yet. Finally, the efficiency and output power of BWOs are inversely proportional to the operating frequency. Even though the BWOs have wide tuneable bandwidth and high spectral purity, the low efficiency prevents their use in a number of 
high frequency applications. This issue exists for most BWOs irrespective of the SWS used. Thus, in order to realize an efficient source for high frequency applications, it is necessary to develop new methods to improve the efficiency of BWOs.

The abovementioned problems provide the motivation for the research reported in this thesis.

\subsection{Objectives}

The main objective of the research presented in this thesis is to study the performance of PH-SEC for microfabrication-compatible millimetre wave BWOs. The goals of the research are as follows:

1) Accurately evaluate the interaction impedance of different space harmonics in a SWS. Based on such evaluation, study the variation of interaction impedance in PH-SEC for different beam positions inside the SWS.

2) Find the dispersion characteristics and interaction impedance of PH-SEC using tape-helix analysis and compare the results with those obtained from 3D electromagnetic simulator such as Computer Simulation Technology (CST).

3) Design and fabricate a PH-SEC SWS with input and output couplers for cold test measurements. Measure the results from the fabricated prototype and compare it with results from simulation and analysis.

4) Design a W-band BWO using microfabrication-compatible PH-SEC. Study the output power, efficiency, and frequency tunability of the BWO.

5) Propose a new technique to improve the efficiency and output power of conventional BWO. Study the efficiency and output power improvement from the proposed technique using simulation.

\subsection{Major Contributions of the Thesis}

i) Introduced a new approach for obtaining fields and interaction impedance for different space harmonics of SWSs. This approach avoids the errors in the existing approaches and provides more accurate values of the fields and interaction impedance. Such results are important for the design of devices such as TWTAs and BWOs and were not available so far.

ii) The cold test parameters like dispersion characteristics and interaction impedance of PH-SEC have been obtained from the tape-helix analysis for the 
first time. The analysis has been simplified by incorporating effective dielectric constant (EDC) method with the tape helix analysis of infinitely wide planar helix. The results from such an analysis show a very good match with those from simulations. Such accurate results are important for the design of devices such as TWTAs and BWOs and were not available so far for the PH-SEC.

iii) A novel BWO operating at W-band has been designed using microfabricationcompatible PH-SEC. The oscillator has been designed to operate with a beam voltage varying from $7 \mathrm{KV}$ to $11 \mathrm{KV}$ and a beam current of $20 \mathrm{~mA}$. The particlein-cell (PIC) simulations show that the frequency of the oscillator can be tuned from $86.9 \mathrm{GHz}$ to $100 \mathrm{GHz}$. The oscillator has a peak efficiency of $1.62 \%$ and has a maximum peak output power of $2.3 \mathrm{~W}$. BWOs have important applications since these provide high spectral purity and electronically tuneable signals.

iv) A new technique has been proposed to improve the efficiency of conventional non-relativistic BWOs without increasing the length of the SWS. The technique has been verified by simulations using the circular helix SWS at Ku-band for three different beam configurations. The simulation results reveal that the proposed technique can improve the efficiency of conventional BWOs by a factor of three to six depending on the beam configuration. The proposed new technique for improvement of BWO efficiency has also been applied to a BWO with PH-SEC as the SWS.

\subsection{Organization of the Thesis}

The thesis has been organized into seven chapters. Chapter 1 gives an introduction to the thesis with brief description of VEDs, BWOs, and SWSs. The motivation behind this research is also presented in this chapter. Existing research on millimetre wave BWOs and the requirement of further research on PH-SEC for developing microfabricated BWO are also explained. Objectives and major contributions of this thesis are also mentioned.

Chapter 2 gives an overview of BWOs. Basic parameters like dispersion diagram, phase velocity, group velocity, space harmonics and interaction impedance of SWSs are introduced in this chapter with a brief review of the measurement methods of various parameters in the SWS. A detailed description of the operating principle of BWOs is presented. Various SWSs for BWOs and the previous work on PH-SEC are also briefly reviewed. A detailed description of the analytical methods for determining dispersion 
characteristics and interaction impedance of circular helix SWS is also given. Finally, the 3D electromagnetic simulation tools for designing TWTs are mentioned.

Chapter 3 start with a brief description of importance of the interaction impedance of different space harmonics in a SWS. Different methods to evaluate the interaction impedance of fundamental space harmonic in a SWS are reviewed. The Fourier decomposition method to determine the magnitude of longitudinal electric field of different space harmonics is also explained. Comparison between the simulation results and analytical results is presented along with the reasons for mismatch between the results. This chapter also provides the interaction impedance of fundamental as well as the $n=-1$ space harmonic of the circular helix.

Field-theory analysis based on the tape-helix approach for determining the dispersion characteristics as well as the interaction impedance of PH-SEC is presented in Chapter 4. Tape-helix analysis of infinitely wide planar helix as well as application of the EDC method are explained. Comparison between the results from analysis, simulation, and sheath-helix analysis in the literature is also presented. This chapter also provides the results of applying the present analysis in determining the dispersion characteristics of rectangular helix. The fabrication and measurement results of PH-SEC in a homogeneous medium are described together with comparison of measured results with those from the tape-helix analysis.

The structure, dispersion characteristics, and transmission characteristics of PH-SEC slow-wave structure for a W-band BWO are presented in Chapter 5. The performance of the designed oscillator is obtained using the particle-in-cell (PIC) simulations. This chapter also provides the output characteristics of the oscillator for three different beam currents. Besides, this chapter presents the design and fabrication of a scaled version of PH-SEC operating at X-band with comparison between measured and simulated results.

Chapter 6 describes the structure and the operating principle of the newly proposed vacuum electronic two-beam oscillator-amplifier. The design and simulation results of the conventional BWO as well as the oscillator-amplifier, both based on the circular helix SWS, are presented. The PIC results of the oscillator-amplifier are obtained for three different beam configurations. The performance of both devices is compared to assess the improvement in the proposed system. Besides, the simulation results for the proposed oscillator-amplifier using PH-SEC are also included in this chapter. 
The thesis is concluded in Chapter 7 by summarizing important results and findings in this research. Some suggestions for future study have also been included in this chapter. 


\section{Chapter 2 Literature Review}

\subsection{Introduction}

Nowadays microwave and millimetre waves are indispensable in various spheres of life and society. Broad-band oscillators are inevitable for widely growing applications which use these frequencies. Backward-wave oscillators (BWOs) are an excellent choice for some of these applications. As mentioned in Chapter 1, a slow-wave structure (SWS) is an important component of BWOs since it determines the electromagnetic properties of the device. This chapter provides a review of the BWOs and different SWSs used for realising these. This chapter also provides a brief review of studies on application of planar helix slow-wave structure with straight-edge connections (PH-SEC) in TWTAs. As mentioned in Chapter 1, PH-SEC is a planar counterpart of the circular helix and is a very promising SWS,

Section 2.2 introduces the basic parameters of SWSs such as phase velocity and interaction impedance. This section also discusses the techniques to estimate these basic parameters. Basic operating principle of three different types of operation of travellingwave tubes (TWTs), viz. TWTA, backward-wave amplifier, and BWO is elaborated in Section 2.3. Different BWOs and the SWS used in them are reviewed in Section 2.4. In Section 2.5, different studies of PH-SEC for TWTA application and its fabrication are reviewed. Section 2.6 briefly introduces the computer aided design tools used in this thesis for designing SWS and BWO.

\subsection{Basic Parameters of Slow-Wave Structure (SWS)}

The electromagnetic (EM) wave travelling through a SWS has different frequency dependent properties such as phase constant, phase velocity, group velocity, and interaction impedance. These parameters can be measured without using an electron beam in the SWS, hence these are called cold-test parameters. The phase constant, phase velocity, and group velocity of an EM wave in a SWS can be obtained from the dispersion diagram of the SWS, hence these parameters are called dispersion characteristics. Accurate evaluation of dispersion characteristics is an important step for the design of SWS for a TWT. Interaction impedance of a SWS is a parameter to express the strength of beam-wave interaction. 


\subsubsection{Dispersion Diagram}

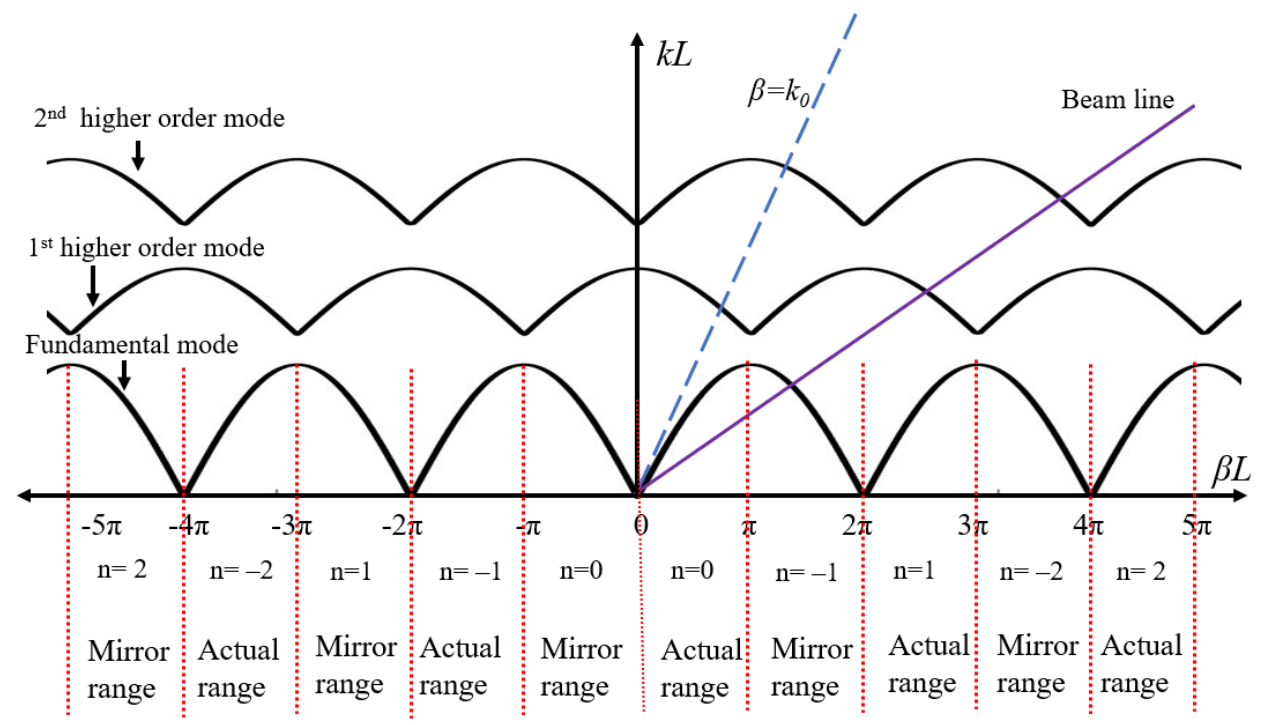

Figure 2.1: Dispersion diagram of a periodic SWS.

Table 2.1 The range of $\beta L$

\begin{tabular}{|c|c|c|c|c|}
\hline \multirow{2}{*}{ Parameter } & \multicolumn{2}{|c|}{$\begin{array}{c}\text { Range of values from } \\
(2.1)\end{array}$} & \multicolumn{2}{c|}{ Range of mirror values } \\
\cline { 2 - 5 } & From & To & From & To \\
\hline$\beta_{0} L$ & 0 & $\pi$ & $-\pi$ & 0 \\
\hline$\beta_{-1} L$ & $-2 \pi$ & $-\pi$ & $\pi$ & $2 \pi$ \\
\hline$\beta_{1} L$ & $2 \pi$ & $3 \pi$ & $-3 \pi$ & $-2 \pi$ \\
\hline$\beta_{-2} L$ & $-4 \pi$ & $-3 \pi$ & $3 \pi$ & $4 \pi$ \\
\hline$\beta_{2} L$ & $4 \pi$ & $5 \pi$ & $-5 \pi$ & $-4 \pi$ \\
\hline
\end{tabular}

The dispersion diagram (also called as $k-\beta$ diagram) of a SWS is the plot between wavenumber $k$ and phase constant $\beta$. The dispersion diagram of a typical periodic SWS is shown in Fig. 2.1. $k L$ versus $\beta L$ is plotted in the figure, where $k=\omega \sqrt{\varepsilon \mu}, \varepsilon=\varepsilon_{0} \varepsilon_{r}, \mu=$ $\mu_{0} \mu_{r}, \omega=2 \pi f$ and $L=$ Pitch. Similar to the waveguides and filters, SWSs also support different modes of propagation. As shown in the figure, the phase constant of a periodic SWS is repetitive along the $\mathrm{X}$-axis; these repeating sets are called space harmonics. The space harmonics are generated due to the spatial periodicity of the SWS. Due to the existence of space harmonics, an EM wave with a single frequency can propagate with different phase constants in a SWS [7]. The phase constants of different space harmonics in a SWS are related as: 


$$
\beta_{n}=\beta_{o}+\frac{2 \pi n}{\text { Pitch }} \quad \text { where } n=0, \pm 1, \pm 2 \ldots \ldots
$$

where $\beta_{n}$ is the phase constant of the $n^{\text {th }}$ space harmonic, $\beta_{0}$ is the phase constant of the fundamental space harmonic, Pitch is the period of a single unit cell, and $n$ is the number of space harmonic. Five different space harmonics $(n=0,-1,+1,-2$ and +2$)$ of the fundamental mode are also marked in Fig. 2.1. The fundamental space harmonic $(n=0)$ and the $n=-1$ space harmonic are the most important space harmonics in a SWS. Taking Pitch= $L$, the range of values of $\beta L$ for a few space harmonics is given in Table 2.1 and is also marked in the dispersion diagram in Fig. 2.1. Following the practice in this field, we use the right-hand side of the dispersion diagram in this thesis.

The phase velocity and group velocity of an EM wave in the SWS can be evaluated from the dispersion diagram. The phase velocity $\left(v_{p}=\omega / \beta\right)$ is the velocity of the phase of the EM wave, whereas the group velocity $\left(v_{g}=d \omega / d \beta\right)$ is the velocity of the energy of the EM wave. The phase velocity at a particular point in the dispersion diagram can be obtained by determining the slope of the line joining the origin and that point in the diagram. The group velocity at a particular point in the dispersion diagram can be obtained by evaluating the slope of the dispersion curve at that point. The free space line $\left(\beta=k_{0}\right)$ is also plotted in the dispersion diagram; the free space velocity is higher than the phase velocity at any point in the fundamental mode.

The phase velocity and group velocity of the electromagnetic wave are in the same direction for the fundamental $(n=0)$ space harmonic, whereas both are in the opposite directions for the $n=-1$ space harmonic. The fundamental space harmonic of a SWS is used for TWTAs whereas the $n=-1$ space harmonic is used for BWOs [35], [36]. The phase constant for the electron beam is represented in Fig. 2.1 using a straight line. BWOs operate at a point where the beam line intersects with the $n=-1$ space harmonic of the SWS. As shown in the figure, the beam line also intersects with the higher order modes, which may create noise in the BWO output.

\subsubsection{Interaction Impedance}

As mentioned earlier, the interaction impedance represents the strength of interaction between the electron beam and the EM wave. This parameter was introduced by Pierce [36]- [38]. The Interaction impedance is also called coupling impedance or Pierce impedance. The interaction impedance is defined as: 


$$
K_{c, n}=\frac{\left|V_{z, n}\right|^{2}}{2 \mathrm{P}}
$$

where $V_{z, n}$ is the longitudinal voltage for $n^{\text {th }}$ space harmonic and $\mathrm{P}$ is the total timeaveraged RF power of signal flowing through the SWS. Equation (2.2) has units of ohms. Also, the interaction impedance is a positive real number.

The definition of interaction impedance leads to the following expression for the interaction impedance for the $n^{\text {th }}$ space harmonic at beam position $(r, \phi)$ :

$$
K_{c, n}=\frac{\left|E_{z, n}(r, \phi)\right|^{2}}{2 P \beta_{n}^{2}}
$$

In (2.3), using the cylindrical coordinate system $(r, \phi, z),\left|E_{z, n}(r, \phi)\right|$ is the magnitude of the longitudinal electric field $\left(\left|E_{z, n}(r, \phi)\right| e^{j\left(\omega t-\beta_{\mathrm{n}} z-\varphi_{0, \mathrm{n}}\right)}\right)$ at beam position $(r, \phi)$ and $\beta_{\mathrm{n}}$ is the phase constant. The derivation of this well-known expression (equation (2.3)) has been included as Appendix A in the thesis.

The following comments elaborate the interpretation and application of interaction impedance:

- The definition of the interaction impedance does not explicitly include parameters of the electron beam. It represents the contribution of the 'wave' in the beam-wave interaction; this contribution depends only on the properties of the SWS or waveguide that supports the electromagnetic wave.

- The expression for the interaction impedance can be appreciated intuitively. For example, consider (2.3). The ratio $\frac{\left|E_{z, \mathrm{n}}(r, \phi)\right|^{2}}{2 P}$ gives the square of the magnitude of longitudinal electric field of the $n^{\text {th }}$ space harmonic as a fraction of the total RF power. A SWS with a higher value of this ratio will lead to a stronger interaction of the $n^{\text {th }}$ space harmonic with a given electron beam. The term $\beta_{n}^{2}$ in the denominator appears due to the sinusoidal variation of the electric field (see derivation in Appendix A).

- Since the interaction impedance depends only on the properties of the SWS or waveguide, its evaluation does not require the parameters of the electron beam (see Sections 2.2.3 and 3.2). Therefore the interaction impedance is classified as a cold-test parameter.

- The interaction impedance enables the designer of a TWT to compare in a quantitative manner the potential of different types of SWSs, or different designs of the same SWS, 
for beam-wave interaction. These are important initial steps in the design of TWTs. It is very convenient that one can complete these steps without explicitly using the beam parameters. Of course, one does consider the beam parameters in the later stages of design.

- As mentioned in Chapter 1 , for beam-wave interaction to occur, the phase velocity $v_{p}$ of the electromagnetic wave travelling through a SWS must be synchronized with the velocity of the electron beam. Since the phase constant of the wave $\beta=\omega / v_{p}$, the beam velocity is included in an implicit manner in the expression for interaction impedance.

- Due to the factor $P$ in the denominator of the expression for interaction impedance, one may either use the actual value or normalize value of $\left|E_{z, n}(r, \phi)\right|$ for calculating the interaction impedance.

\subsubsection{Estimation Methods}

Analysis, simulation, and measurement are the different methods used to determine the dispersion characteristics as well as the interaction impedance of a SWS. Field theorybased analysis and equivalent circuit analysis are two of the analytical methods. The equivalent circuit analysis considers the SWS as a transmission line and evaluates its equivalent capacitance $C$ e and inductance $L$ e. The phase constant $\beta$ of the SWS is calculated as $\beta=\omega \sqrt{ } C_{e} L e$ [39], [40]. The field theory-based analyses use Maxwell's equations with appropriate boundary conditions to obtain the dispersion characteristics of the SWS. The field theory approach was initially applied to circular helix SWS by Pierce. He replaced the circular helix with an equivalent sheath-helix structure [41], [42]. The sheath-helix approximation does not consider the effects of space harmonics. Later, tape-helix approach was proposed by Sensiper to consider the effects of space harmonics and tape width of the circular helix SWS [43].

Although the analysis is the fastest method to obtain the dispersion characteristics, simplifying assumptions in the analysis may cause errors in the results. In the last two decades, computer simulation methods have evolved for calculating the dispersion characteristics as well as the interaction impedance of SWSs. Different simulation software like Computer Simulation Technology (CST), Ansys High Frequency Structure Simulator (HFSS), and COMSOL are commonly used for studying the dispersion characteristics of the SWSs 
Measurement is the complex and time-consuming method to find various parameters of SWS. The dispersion characteristics of SWSs are usually measured using two identical slow-wave structures with different lengths. Difference in the phase of transmission characteristics in these two SWSs is used for finding the phase constant of a unit cell. Resonance method is also used for finding the dispersion characteristics in the SWS; this method determines the resonant frequencies of a short-circuited length of slow-wave structure [44], [45].

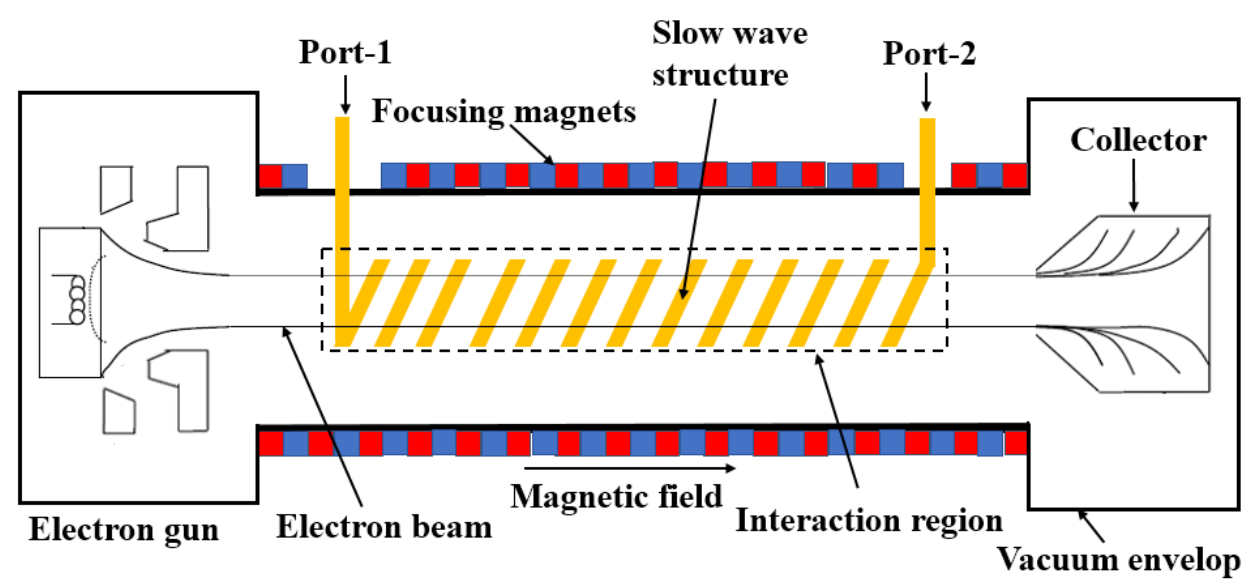

Figure 2.2: Schematic diagram of a travelling-wave tube.

The interaction impedance of the fundamental space harmonic in a SWS can be measured using resonant or non-resonant perturbation techniques. Resonant perturbation technique determines the interaction impedance by measuring the change in the resonance frequency due to insertion of a dielectric cylinder into the short-circuited length of slowwave structure [44]. Non-resonant perturbation technique measures the change in the phase constant due to insertion of a dielectric cylinder into the SWS for determining the interaction impedance [46]-[48]. The abovementioned measurement techniques do not use an electron beam for measurement; hence these are called cold-test measurements. The interaction impedance of a SWS can also be measured using electron beams; such measurements are called hot-test measurements [49].

\subsection{Principle of Operation of TWTs}

The schematic diagram of a typical TWT is shown in Fig. 2.2. TWTA, backwardwave amplifier (BWA), and BWO are the three different modes of operation of TWTs. The three modes of operation differ only in the function of ports and the speed of the electron 
beam. The interaction of electron stream with the EM wave in a slow-wave structure in vacuum environment was first observed by Lindenblad [50]. He developed a primitive form of TWTA in which the input signal was used for the modulation of the electron beam. Later, R. Kompfner developed a wide band amplifier in which the input signal was fed directly to the helix [51], [52]. Practical TWTAs were developed using different SWSs and analysed by J.R. Pierce and L.M. Field [36], [37], [53].

Discovery and subsequent study of backward-wave oscillations in TWTAs led to the development of BWOs. R. Kompfner and N. T. Williams observed backward-wave amplification and oscillation in TWTs and they found that the tube can operate as an electronically tuneable amplifier or oscillator. Kompfner gave simplified results for starting current and gain of BWO. Later, Pierce's traveling-wave theory was used for detailed analysis of starting current, loss, efficiency, and effect of space charge in a BWO [9], [54][56]. The following sub-sections provide a description of the operation of, and difference in, the three modes of operation of TWTs.

\subsubsection{Travelling-Wave Tube Amplifier (TWTA)}

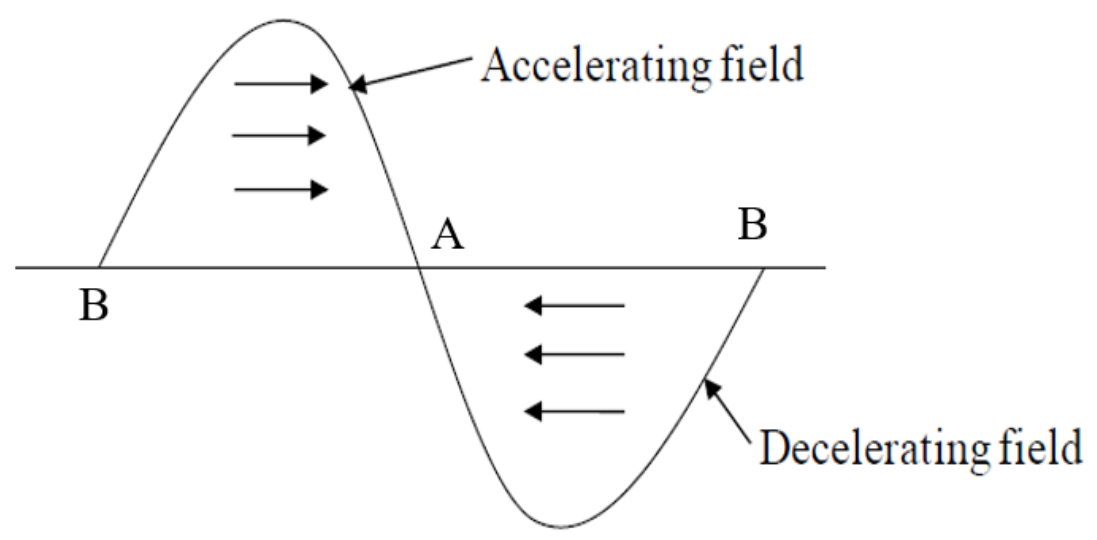

Figure 2.3: Axial electric field for velocity modulation.

The TWTA is a widely used vacuum electronic amplifier due to its wide bandwidth, high power, high gain, and high efficiency. TWTAs have been developed using various SWSs like circular helix, corrugated wave guide, sine waveguide, folded waveguide and coupled cavities [57]-[61]. TWTAs have been developed for frequencies ranging from below $1 \mathrm{GHz}$ to above $100 \mathrm{GHz}$. Now researchers are focusing on developing microfabrication-compatible TWTAs for realising amplifiers operating at $100 \mathrm{~s}$ of GHz and above. TWTAs can work in the continuous wave mode with output power range from a 
few milli watts to thousands of watts. Besides, the peak pulse power of pulsed TWTAs can reach over a megawatt. The efficiency of TWTAs used in satellites usually exceeds $60 \%$.

As mentioned previously, TWTAs use the fundamental space harmonic of the SWS. In common SWSs like circular helix, the fundamental space harmonic has a constant phase velocity for a wide frequency range. Therefore, a single beam velocity can attain velocity synchronization for a broad bandwidth. For a TWTA, port-1 in Fig. 2.2 is used for feeding the input signal whereas the output signal is coupled out through port-2.

The input signal at port-1 of the SWS propagates as different space harmonics. Here, we are interested only in the fundamental space harmonic. The axial electric field corresponding to the fundamental space harmonic is the only field that helps in the beamwave interaction. The axial electric field in a SWS is shown in Fig. 2.3. As the electron beam travels through the SWS, it experiences an accelerating and decelerating force from this electric field. This causes velocity modulation of the electrons. The velocity modulation forces the electrons to group together at locations marked as point $\mathrm{A}$ in each period. The electrons in and around the locations marked as point $\mathrm{B}$ move to locations $\mathrm{A}$, hence forming electron bunches. This process continues throughout the SWS. The movement of electron bunches is similar to the flow of an alternating current; hence the formation of bunches is also called as current modulation. Once the bunches are formed, the EM wave in the SWS extracts energy from these and grows in amplitude. The speed of EM wave must be slightly slower than the speed of electrons in order to generate a growing field in the SWS. In a TWTA, the formation of bunches and extraction of energy are a continuous process and it occurs throughout the length of the SWS.

\subsubsection{Backward-Wave Amplifier (BWA)}

The BWA is a narrow band amplifier employing the $n=-1$ space harmonic of the SWS [7]. The input signal for this amplifier is applied to port-2 and the amplified output signal is taken out of port- 1 . Since the BWA employs the $n=-1$ space harmonic, it needs a lower beam voltage than that of the TWTA.

When an input signal is fed to the BWA, it propagates through the SWS as different space harmonics. The $\mathrm{n}=-1$ space harmonic and electron beam attain velocity synchronization. As a result of this interaction, beam bunches are formed and flow to port2. The kinetic energy of the electron bunches is transferred to the EM wave and the energy of the signal grows as it flows to port- 1 . The phase of $n=-1$ space harmonic propagates in 
the direction of electron beam (from port-1 to port-2) whereas the direction of energy flow is in the opposite direction of electron beam. The strength of the electron bunches in a BWA is maximum at a point near the collector whereas the signal strength is the lowest at this place. Thus, a BWA fails to extract maximum energy from the electron bunches. On the other hand, in a TWTA, the signal strength and electron bunches grow in the same direction, which gives it a higher efficiency. Further, a particular beam voltage in a BWA can synchronize with a single frequency only. Hence this amplifier has a narrow bandwidth. BWAs are not commonly used due to their low efficiency and narrow bandwidth.

\subsubsection{Backward-Wave Oscillator (BWO)}

The gain of BWAs increases with the beam current and goes to infinity at a particular beam current called the starting current. The output of a BWA starts to oscillate at a beam current higher than the starting current and the BWA converts to a BWO. An input signal is not required for the operation of a BWO; like other oscillators, BWO also starts its oscillation by amplifying the noise signal. The frequency of oscillation in a BWO can be tuned by changing the beam voltage. The change in beam voltage can tune the frequency at which the velocity synchronization occurs between the electron beam and $n=-1$ space harmonic. Low DC-RF conversion efficiency is the main drawback of the BWO.

In the early stages of BWO development, several researchers used small signal theory to estimate the starting current of BWO. Johnson carried out the analysis for a limited range of circuit loss and limited space charge force [9]. (The space charge force is the Coulomb repulsive force which can cause the electrons in the electron beam to disperse in the radial direction. This force is proportional to the charge density of the beam). Other researchers carried out the analysis for starting current under various conditions [54]-[56], [62]. The analytical results for starting current at different conditions were combined to get an equation for starting current for large loss and large space charge condition in [62]. The efficiency of BWO has also been analysed using small signal theory [55]. Analysis shows that the efficiency and starting current of BWOs depends on the interaction impedance, interaction length, space charge force, circuit loss, beam thickness, and velocity spread. The following section provides a brief review of the various BWOs reported in the literature.

\subsection{Backward-Wave Oscillators}

BWOs can be classified according to their operating voltage as relativistic BWOs and low voltage BWOs. The relativistic BWOs uses comparatively high beam voltage such 
as hundreds of $\mathrm{KV}$ whereas beam voltage of a few $\mathrm{KV}$ is used in the low voltage BWOs.

Normally relativistic BWOs are preferred for high power pulsed operations and low voltage BWOs are used for low power continuous wave $(\mathrm{CW})$ operation. The relativistic BWOs have electron beam velocity closer to the speed of EM wave in free space. Moreover, the relativistic BWOs require relatively high magnetic field for focusing beams with high current density. Generation and focusing of relativistic electron beam are the main challenges in this device. The following sub-sections gives some examples of the relativistic BWOs as well as the low voltage BWOs.

\subsubsection{Relativistic BWOs}

Relatively high peak power of $140 \mathrm{MW}$ for $300-400$ ps pulses at $38 \mathrm{GHz}$ have been attained from a relativistic BWO [63]. This relativistic BWO uses an electron beam with beam voltage of $240 \mathrm{KV}$ and beam current of $1.6 \mathrm{KA}$ to produce EM pulses with a maximum efficiency of 5\%. Another relativistic BWO which can generate pulses with power of up to $400 \mathrm{MW}$ at $38 \mathrm{GHz}$ with a maximum efficiency of $20 \%$ has been reported in [64]. This BWO uses a beam current of $2 \mathrm{kA}$ and beam voltage of $300 \mathrm{KV}$. A relativistic BWO which produces pulse power of $1.1 \mathrm{GW}$ at $9.38 \mathrm{GHz}$ by using a relativistic beam with beam voltage of $610 \mathrm{KV}$ and beam current of $5.4 \mathrm{KA}$ has been reported in [65]. This BWO has a maximum power transfer efficiency of 33\%. Corrugated SWSs are commonly used in relativistic BWOs due to their high-power handling capacity. Sinusoidally corrugated circular waveguide has been used for the design and experimental demonstration of a 0.34-THz BWO [11]. Experimental studies show that the BWO can produce pulse radiation with peak RF output power of $600 \mathrm{KW}$ and a pulse width of 1.66 ns, which corresponds to a peak efficiency of $0.67 \%$. The BWO has been driven by a weakly relativistic electron beam with beam voltage of $100-\mathrm{kV}$, and beam current of $900-$ A. The frequency range of output signal in this device varies from $320 \mathrm{GHz}$ to $340 \mathrm{GHz}$.

Plasma filling is common in relativistic BWOs due to the significant improvements offered by plasma. Plasma filling helps in increasing the electron beam current, bandwidth, and efficiency of relativistic BWOs. Moreover, plasma helps in the reduction or elimination of the need for focusing magnetic fields in these devices. Plasma enhances the current capability and beam propagation by the neutralization of the space charge in electron beam [66]-[68]. Increased noise and instability in the output signal are the main drawbacks of plasma filled BWOs. 
BWOs with relatively high beam voltage have also been used for continuous wave operation. Metallic waveguides with corrugations or gratings are used as the SWS for handling such high-power electron beams. Even though the metallic SWSs have high power and high temperature handling capability, they have a lower slowing down factor and a smaller frequency bandwidth compared with that of a circular helix. Rectangular double grating SWS has been used for designing a continuous wave BWO which uses a beam voltage of $141 \mathrm{KV}$ and beam current of $168 \mathrm{~A}$; the BWO has a maximum efficiency of $19 \%$ at $31.68 \mathrm{GHz}$ and a tuneable bandwidth of 7\% [69]. Another continuous wave BWO has been designed with corrugated cylindrical waveguide, using an annular beam with beam voltage of $340 \mathrm{KV}$ and beam current of 30 A [70]. Deep corrugations and pitch tapering have been used in this BWO to improve the peak efficiency to $58 \%$ at $28 \mathrm{GHz}$. Significant reduction in tuneable bandwidth occurs for this BWO due to the efficiency improvement techniques.

Development of relativistic BWOs is challenging and costly due to the requirement of relativistic electron beam and relatively high focusing magnetic field. The output powers of relativistic BWOs are in the order of megawatts and hence not useful for low power or medium power applications. The relativistic BWOs are not suitable as airborne or space borne devices since they have quite large sizes. Therefore, development of low voltage BWOs is required for low and moderate power applications. Besides, low voltage devices have advantages like low cost and light weight.

\subsubsection{Low Voltage (Non-Relativistic) BWOs}

Low voltage BWOs have been developed using different SWSs like circular helix, folded waveguide, sine waveguide, corrugated waveguide, etc. Folded waveguide SWS has been used for designing a low voltage BWO at $95 \mathrm{GHz}$ with a maximum efficiency of $0.84 \%$. This BWO uses an electron beam with $12 \mathrm{KV}$ beam voltage and $50 \mathrm{~mA}$ beam current [25]. Staggered double-vane SWS has been used for developing a BWO with watt level of output power at $90 \mathrm{GHz}$. This BWO requires a beam voltage of $10 \mathrm{KV}$ and a beam current of $200 \mathrm{~mA}$ [71]. Staggered double-vane SWS has also been used for developing a BWO with maximum efficiency of $1.77 \%$ at $140 \mathrm{GHz}$. The BWO uses a sheet electron beam with a beam voltage of $20 \mathrm{KV}$ and beam current of $10 \mathrm{~mA}$ [14]. A BWO is designed using folded waveguide SWS to operate at $220 \mathrm{GHz}$ [15]. The experimental studies show that the oscillator can operate between $227.45 \mathrm{GHz}$ and $228.74 \mathrm{GHz}$ by varying the beam 
voltage between $16.76 \mathrm{KV}$ and $17.35 \mathrm{KV}$. This oscillator generates a maximum output power of $16.2 \mathrm{~mW}$, which corresponds to a DC-to-RF conversion efficiency of $0.008 \%$. A helical BWO has been developed at $650 \mathrm{GHz}$ to operate with $7 \mathrm{KV}$ beam voltage and $7 \mathrm{~mA}$ beam current. The developed BWO is predicted to give an output power of $60 \mathrm{~mW}$ with an efficiency of $0.12 \%$ [10]. Recently, researchers have been trying to develop low voltage BWOs which can generate signals with frequencies of THz and above [72], [73]. Relatively low efficiency is the main disadvantage of low voltage BWOs.

The studies mentioned above suffer from relatively low tuneable bandwidth and low efficiency. In comparison, the BWO based on PH-SEC reported in Chapter 5 achieves a wider tunable bandwidth of $\sim 14 \%$. The technique reported in Chapter 6 achieves an efficiency which is higher by a factor of three to six compared to conventional designs.

\subsection{Previous Studies on PH-SEC}

The circular helix is the most commonly used SWS in TWTs due to its wide bandwidth and strong beam wave interaction. But its geometry is not easily amenable to microfabrication and mass production. In this context, research on planar SWSs that can be microfabricated becomes very important. PH-SEC is a microfabrication-compatible planar version of circular helix. The PH-SEC has been proposed and its dispersion characteristics have been studied in [31]. Significant amount of research has already been done on the development and study of PH-SEC for TWTA application [29]-[32] [74], [75]. The following sub-sections provide a brief review of the studies on PH-SEC for TWTA applications.

\subsubsection{Dispersion Control}

The dispersion characteristics of SWS have a strong impact on the performance of TWTAs. SWSs with nondispersive fundamental space harmonic are desirable for wide bandwidth TWTAs. Metal vanes are commonly used for dispersion-shaping for the circular helix. Three types of metal wanes namely T-shaped, solid, and thin vanes have been found very effective for circular helix. The impact of such metal vanes on the characteristics of fundamental space harmonics in PH-SEC has been studied [74]. The studies show that, similar to the case of circular helix, a flatter dispersion curve or negative dispersion characteristics can be obtained from PH-SEC using metal vanes. Other than metal vanes, coplanar ground planes in PH-SEC also have significant effect on dispersion. The 
simulation and measurement studies show that stronger dispersion control can be achieved from a PH-SEC by using metal vanes together with extended coplanar ground planes [74].

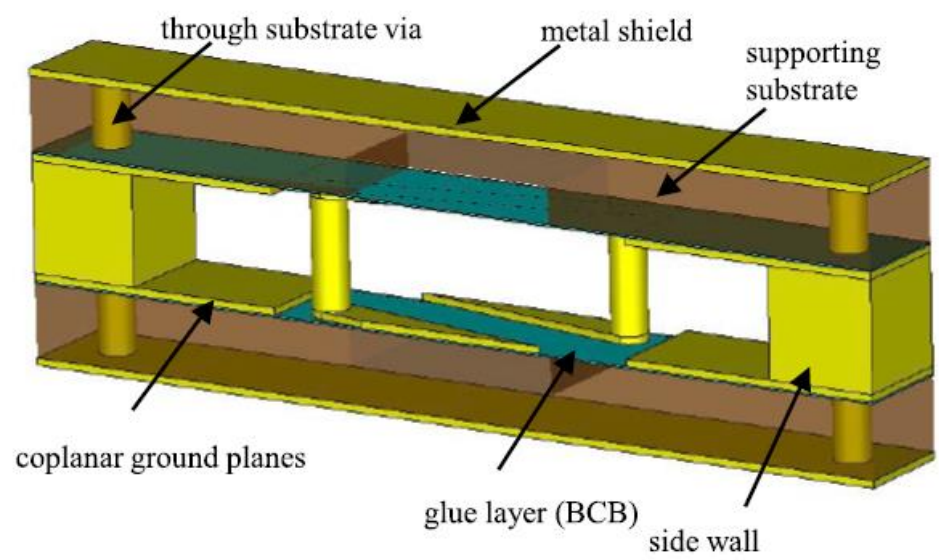

Figure 2.4: Perspective view of one period of the microfabrication-compatible PH-SEC [32].

The studies on dispersion control lead to the development of microfabricationcompatible PH-SEC SWS shown in Fig. 2.4 [32]. The PH-SEC is supported using dielectric substrates for ease of fabrication. Coplanar ground planes and metal shielding are used for dispersion control. Microfabrication-compatible dielectric materials are used in the design.

\subsubsection{Application in TWTA}

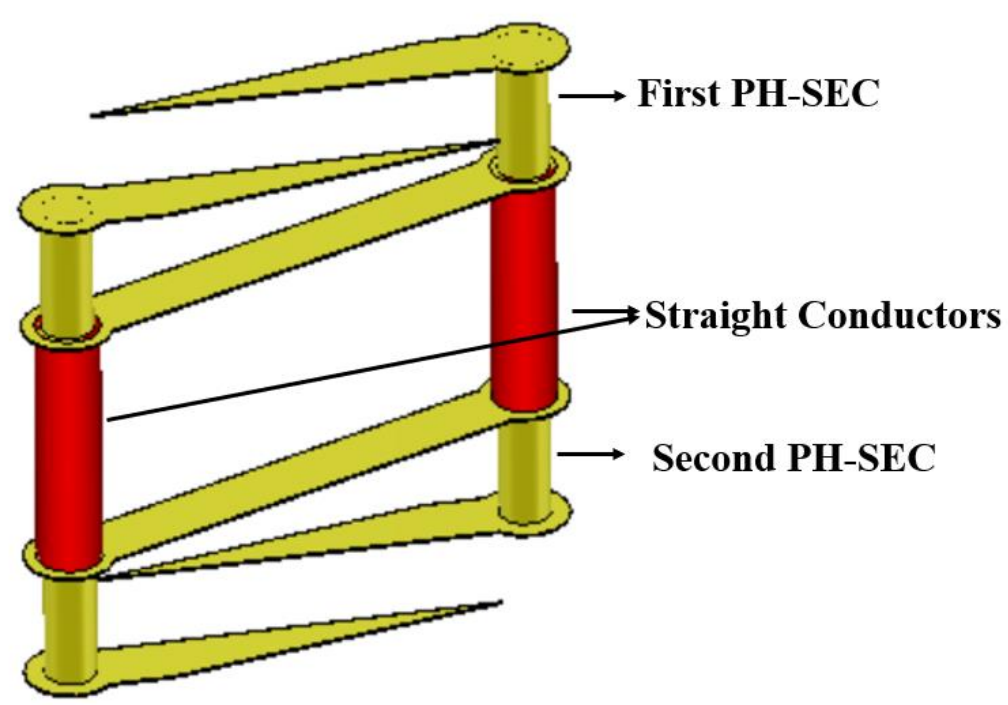

Figure 2.5: Perspective view of one period of the connected pair of PH-SECs [76].

The performance of a Ka-band TWTA based on microfabrication-compatible PHSEC has been studied using PIC simulations [75]. The simulation results show that the PH- 
SEC based TWTA can give an octave bandwidth centred at $30 \mathrm{GHz}$. The designed TWTA uses an electron beam with beam voltage of $3.75 \mathrm{KV}$ and beam current of $10 \mathrm{~mA}$ to get a saturated output power of $6.65 \mathrm{~W}$ at $30 \mathrm{GHz}$ and a small-signal gain of $36 \mathrm{~dB}$. Another study on microfabrication-compatible PH-SEC for TWTA application is described in [32]. The TWTA is designed to use an elliptical cross-sectional electron beam with beam voltage of $3.72 \mathrm{KV}$ and current of $50 \mathrm{~mA}$. The PIC simulation results show that the TWTA gives a bandwidth of $48.5 \%$ and a maximum gain of $42 \mathrm{~dB}$ at Ka-band. The TWTA gives a saturated power of $26.5 \mathrm{~W}$.

Unwanted backward-wave oscillations due to the interaction of the beam with some backward-wave space harmonic is the main source of noise in TWTA output. In this context, connected pair of PH-SEC has been proposed for reducing the risk of backward-wave oscillation compared to that in a single PH-SEC [76]. As shown in Fig. 2.5, the connected pair of PH-SECs is realized by connecting two PH-SECs with straight conductors. Studies shows that the connected pair of PH-SEC has a lower interaction impedance for the backward-wave space harmonics and higher interaction impedance for the fundamental space harmonic. Accordingly, the connected pair of PH-SECs has a higher gain growth rate than that for the single PH-SEC.

\subsubsection{Fabrication of $\mathrm{PH}-\mathrm{SEC}$}

The fabrication of $\mathrm{PH}-\mathrm{SEC}$ has been demonstrated using printed-circuit technique as well as microfabrication technique [29], [30]. The printed-circuit fabrication of PH-SEC at $C / X$-band frequencies using vacuum and high-temperature compatible materials is presented in [30]. The SWS has been fabricated on a pair of vacuum compatible alumina substrates using printed-circuit techniques. High temperature soldering materials have been used to realize the straight-edge connections in the PH-SEC. The SWS has been fixed inside a nonmagnetic steel housing and coaxial couplers have been used for feeding the SWS. Laser welding of the steel housing has been used for achieving a vacuum-tight shield. The PIC simulation of the SWS shows that the fabricated structure can operate in the frequency range of 6-8 GHz with a saturation output power of $100 \mathrm{~W}$. PH-SEC at W-band with coplanar waveguide feed has been fabricated using microfabrication technique [29]. Onwafer cold test measurements of the fabricated SWSs have been carried out and the measured and simulated results match well. The effects of silicon wafer resistivity, copper conductivity, and surface roughness in fabricated SWS have also been studied. 


\subsection{Simulation Tools for BWO Design}

Field-theory based analyses have been used for studying the properties of SWSs and BWOs. Such analyses use simplifying assumptions to replace the original structure by an easily solvable structure. The error produced by the simplifying assumptions is the main drawback in theoretical analysis. Besides, theoretical analyses may not be suitable for finding the characteristics of complex SWSs.

On the other hand, simulation tools can provide accurate results for complex structures and can take into account practical issues such as lossy conductors, non-zero conductor thickness, and complicated shapes. Recent development of simulation software and high-power computers reduces the difficulty in obtaining the high frequency characteristics of EM structures. The dispersion characteristics and interaction impedance of the SWSs can be obtained using simulations. Moreover, the performance of TWTs (including power, efficiency, and output frequency spectrum) can also be predicted using simulations. In view of these features, simulation tools have become vital for the design of TWTs such as BWOs.

A number of simulators have been developed to solve the Maxwell's equations for 3D structures with arbitrary geometries. Mainly, time-domain and frequency domain approaches are used in these software. Finite difference time-domain (FDTD), transmission-line-matrix (TLM), and finite integration technique (FIT) are the commonly used methods for time-domain approach. The finite element method (FEM) is the wellknown method for the frequency domain approach.

Computer Simulation Technology (CST) Microwave Studio (MWS) [77], Ansoft High Frequency Structure Simulator (HFSS) [78] and FEKO [79] are the most commonly used commercial simulation tools for finding dispersion characteristics and interaction impedance of SWSs. Moreover, the Particle-in-Cell (PIC) simulation software like ARGUS [80], MAGIC [81], Christine [82], VORPAL [83], and CST PIC [77] have been widely used for finding the performance of BWOs. We have used CST MWS and CST Particle-in-Cell (PIC) for this research.

CST MWS is a software package for the analysis and design of electromagnetic structures for high frequency applications. This software can support different types of meshes like hexahedral mesh, tetrahedral mesh, etc. Both time-domain and frequency domain approaches are available in this software. Advanced features like thermal simulation, 
dielectric breakdown simulation, etc. are also available. This software provides a 3D modelling frontend based on ACIS modelling kernel. After construction of the model, the mesh cells for simulation are automatically generated in the software. Eigenmode solver and transient solver are the frequently used solvers for the design of SWSs. The eigenmode solver is used for obtaining different operating modes in a resonant structure. The eigenmode solver does not need the results of scattering parameters for obtaining the dispersion characteristics of a SWS. Periodic boundary condition can be applied in eigenmode solver; hence this solver requires only the model of a unit cell to obtain the dispersion characteristics and interaction impedance. The transient solver is used for getting the scattering parameters of the SWS for a broad frequency range. This solver is based on finite integration method together with some highly advanced techniques which increase the accuracy of simulations substantially.

The interaction between electrons in the beam and electromagnetic field can be simulated using CST PIC solver. In this solver, a number of electrons are modelled as a particle for reducing the simulation time. This solver has the provision to define the focusing magnetic field for TWTs. The BWOs' output performance like efficiency and power can be obtained using this solver.

\subsection{Summary}

In this chapter, basic parameters of SWSs, such as dispersion characteristics and interaction impedance are introduced. The methods for evaluating these parameters are also briefly reviewed. The review brings out the importance of accurate determination of interaction impedance for various modes in a SWS. Next, the basic operating principle and the difference between different types of operation of TWT devices are introduced. Different SWSs used for realising BWOs are briefly mentioned. There exists a clear need of investigation of high frequency microfabrication-compatible SWSs for realizing BWOs. It is also made clear that improvement of efficiency of BWOs is very desirable. In this context, the literature on PH-SEC SWS has been briefly reviewed with some details of dispersion control, application in TWTs, and fabrication methods. This chapter concludes with a brief introduction to the common simulation tools as well as the tool used in this research for designing SWSs as well as BWOs. 


\section{Chapter 3 INTERACTION IMPEDANCE OF NON- FUNDAMENTAL SPACE HARMONICS}

\subsection{Introduction}

Design and development of travelling-wave tubes (TWTs) are essential for wide range of applications which use millimetre wave frequencies and above. An accurate estimate of the interaction impedance for the fundamental $(n=0)$ and non-fundamental space harmonics of the slow-wave structures (SWSs) is required for the design of TWTs. The interaction impedance of fundamental space harmonic is required to calculate the gain and saturation length of travelling-wave tube amplifier (TWTA). The interaction impedance of $n=-1$ space harmonic is required to evaluate the minimum length and starting current of backward-wave oscillators (BWOs). Moreover, the possibility of unwanted oscillations in TWTAs can be assessed by comparing the interaction impedance of the fundamental and the non-fundamental space harmonics of the SWS. Commonly, on-axis value of the interaction impedance is used for calculations, but off-axis values of the interaction impedance are also required in many situations. This chapter presents the simulation based method for evaluating the interaction impedance of fundamental and nonfundamental space harmonics in a SWS at on-axis and off-axis positions. Limitations of the analysis for evaluating the interaction impedance of non-fundamental space harmonics are also discussed in this chapter.

Section 3.2 of this chapter gives a review of different methods to evaluate the interaction impedance of fundamental space harmonics in a SWS. This section describes the analysis of the circular helix using the tape-helix approximation. This section also includes a brief discussion on the measurement vs. simulation methods. The magnitude of longitudinal electric field for different space harmonics and the phase constant of the circular helix SWS from simulation are compared with those from analysis. The results of the comparison are presented in Section 3.3. The circular helix is used for the comparison since it has readily available and widely accepted analytical models. Section 3.3 also discusses the limitations of the tape-helix analysis by assessing the validity of simplifying assumptions in the analysis. The simulation method proposed to determine the interaction impedance of non-fundamental space harmonics is presented in Section 3.4. This section also gives the interaction impedance for the fundamental as well as the $n=-1$ space 
harmonic of the circular helix SWS. Finally, the summary and important conclusions of this chapter is given in the section 3.5 .

\subsection{Methods to Find the Interaction Impedance of the Fundamental Space Harmonic}

As mentioned in Chapter 2, the interaction impedance of a SWS is an important parameter which gives the strength of interaction between electron beam and electromagnetic (EM) wave. The interaction impedance for the $n^{\text {th }}$ space harmonic of a periodic SWS is given as:

$$
K_{c, n}=\frac{\left|E_{z, \mathrm{n}}(r, \phi)\right|^{2}}{2 P \beta_{n}^{2}}
$$

where $\left|E_{z, n}(r, \phi)\right|$ denotes the magnitude of the longitudinal electric field for the $n^{\text {th }}$ space harmonic at the position $(r, \phi)$ and $\mathrm{P}$ is the time-averaged RF power. $\beta_{n}$ is the phase constant for the $n^{\text {th }}$ space harmonic, which is related to the phase constant of the fundamental space harmonic $\left(\beta_{o}\right)$ as:

$$
\beta_{n}=\beta_{o}+\frac{2 \pi n}{\text { Pitch }}
$$

Field theory-based analysis, measurement, and simulation are the three methods for evaluating this parameter. The following sub-sections briefly describe these methods.

\subsubsection{Analytical Modelling}

The field theory-based analysis usually approximates the physical structure of the SWS into an approximate model for reducing the complexity. The sheath-helix and the tape-helix approximations are the widely used simplifications for the field theory-based analysis of the SWSs. These approximations were initially developed for finding the dispersion characteristics and the interaction impedance of the circular helix SWS [41], [43]. Later on, such analyses were also applied to different SWSs such as contra-wound helix, ring-bar helix, and rectangular helix [84]-[88]. The characteristic equation for the approximate model in the analysis is derived using Maxwell's equations and appropriate boundary conditions. The characteristic equation so obtained is numerically solved to find the phase constant of the SWS at different frequencies. Subsequently the field expressions and the time-averaged RF power of the SWS are calculated using the phase constant. Finally, the interaction impedance can also be obtained from these analyses using (3.1). 
Both sheath-helix and tape-helix approximations were first introduced for the circular helix in free space. Later on, the effect of dielectric support and metal enclosure for the helix was considered by Tien and McMurtry [89], [90]. After that, the circular helix with inhomogeneous loading was analysed using both sheath-helix and tape-helix approximations in [91], [92], respectively. The circular helix with more practical considerations like vane-loading, T-shaped dielectric support, etc. have also been analysed using field theory-based analyses [93], [94]. The following sub-sections provide a brief introduction to the analysis of circular helix in free space based on sheath-helix and tapehelix approximations. The description is limited to the derivation of characteristic equation since the remaining steps are straightforward.

\subsubsection{Sheath-HeliX ANAlysis}

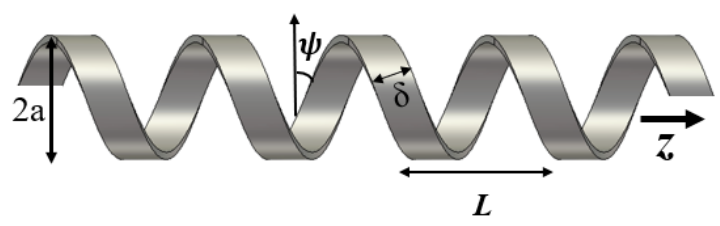

(a)

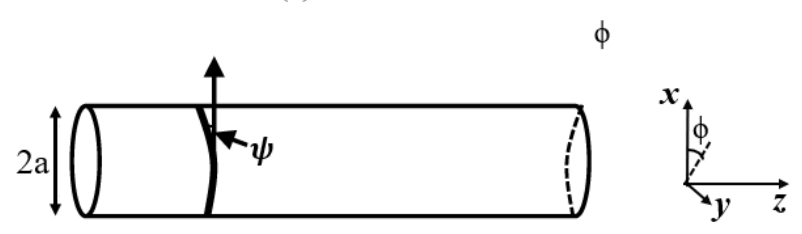

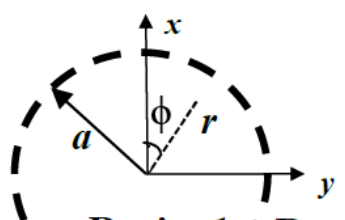

1 Region1 / Region 2

(c)

(b)

Figure 3.1: (a) circular helix; (b) Sheath-helix model; (c) Side view of the sheath-helix model.

As shown in Fig 3.1 (a), the circular helix is represented using cylindrical coordinates $(r, \phi, z)$. The circular helix consists of a tape with finite thickness wound into a helical shape with pitch length $(L)$. The sheath-helix approximation replaces this structure by a circular cylindrical sheath with infinitesimal thickness as shown in Fig 3.1 (b). The radius of sheath-helix is equal to the mean radius of the actual helix. The sheath-helix is assumed to have an anisotropic conductivity: infinite conductivity in the direction parallel to the winding and zero conductivity in the direction perpendicular to the winding. The sheath-helix model would be closer to the actual circular helix if tape width is very small and the helix period is small. The sheath-helix model is valid only when the pitch length $(L)$ of the helix is much smaller than the wavelength. 
The sheath-helix analysis does not consider the effect of space-harmonics. The EM wave is assumed to be propagating in the $z$-direction with a phase constant $\beta$; hence all the field expressions have a variation of $e^{j(\omega t-\beta z)}$. Due to anisotropic conductivity of sheathhelix, both transverse electric (TE), and transverse magnetic (TM) modes can be present in the structure. Using Maxwell's equations, the axial electric $\left(E_{z}\right)$ and magnetic $\left(H_{z}\right)$ fields in the sheath-helix can be expressed in cylindrical coordinates as [41]:

$$
\begin{array}{ll}
E_{z 1}=A I_{0}(\gamma r) e^{j(\omega t-\beta z)} & (r \leq a) \\
E_{z 2}=B K_{0}(\gamma r) e^{j(\omega t-\beta z)} & (r \geq a) \\
H_{z 1}=C I_{0}(\gamma r) e^{j(\omega t-\beta z)} & (r \leq a) \\
H_{z 2}=D K_{0}(\gamma r) e^{j(\omega t-\beta z)} & (r \geq a) .
\end{array}
$$

where $E_{z 1}$ and $H_{z 1}$ are the axial electric and magnetic fields in region 1 whereas $E_{z 2}$ and $H_{z 2}$ are the fields in region 2 (see Fig. 3.1(c)); $k_{0}$ is the free space wavenumber and $\gamma=$ $\sqrt{\beta^{2}-k_{0}^{2}} \cdot I_{0}$ and $K_{0}$ are the zeroth order modified Bessel functions of the first and second kind, respectively. $A, B, C$, and $D$ are four unknown amplitude constants. All other components of electric and magnetic fields $\left(E_{r}, E_{\phi}, H_{r}\right.$, and $\left.H_{\phi}\right)$ in the two regions of the sheath-helix can be simply obtained from $E_{z}$ and $H_{z}$ by using Maxwell's equations. Four independent boundary conditions are used for finding the unknown constants $A$ to $D$ in the field expressions. The boundary conditions at the boundary of helix $(r=a)$ are:

$$
\begin{aligned}
& E_{\phi 1}=E_{\phi 2} \\
& E_{Z 1}=E_{Z 2} \\
& E_{\phi 1} \cos \psi+E_{Z 1} \sin \psi=0 \\
& H_{\phi 1} \cos \psi+H_{Z 1} \sin \psi=H_{\phi 2} \cos \psi+H_{Z 2} \sin \psi
\end{aligned}
$$

The characteristic equation for the sheath-helix model can be obtained by considering the condition of non-trivial solution while applying boundary conditions as:

$$
\frac{k_{0} \cot \psi}{\gamma}=\left(\frac{I_{0}(\gamma a) K_{0}(\gamma a)}{I_{1}(\gamma a) K_{1}(\gamma a)}\right)^{1 / 2}
$$

where $I_{l}$ and $K_{l}$ are the first order modified Bessel functions and $\psi$ is the pitch angle of the helix, given as $\psi=\tan ^{-1}\left(\frac{L}{2 \pi a}\right)$. 
The phase constant obtained by solving (3.5) is used for finding the field expressions, and the interaction impedance of the fundamental space harmonic.

\subsubsection{TAPE-HELIX ANALYSIS}

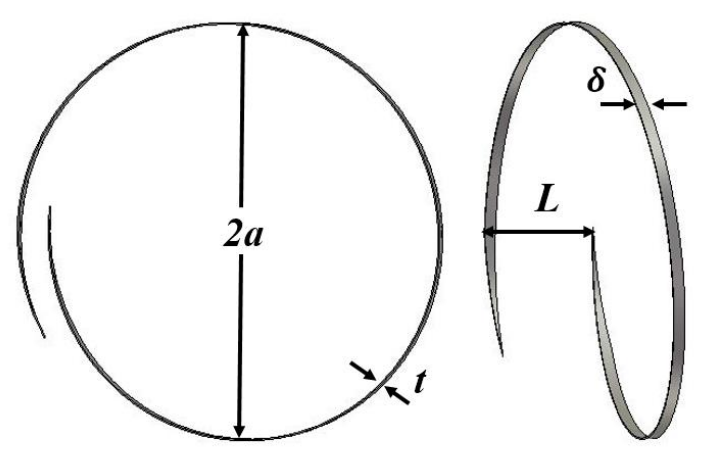

Figure 3.2: Circular tape-helix.

As shown in Fig. 3.2, the tape-helix analysis considers the helix as a perfectly conducting tape with finite width and infinitesimal thickness $(t \rightarrow 0)$. The helix is considered to have an inner radius of $a$. The width of the tape $(\delta)$ is considered as much smaller than the helix pitch length $(L)$. This analysis assumes that the current in the helix is concentrated on the surface of the tape and flows only in the direction of the winding. The conductivity at the surface of the tape $(r=a)$ in the direction parallel to the winding along centre-line of the tape is considered as infinite; hence the electric field intensity at this line is taken as zero.

\section{A. General field expressions}

The wave is assumed to be propagating in the $z$-direction with a field variation of $e^{j(\omega t-\beta z)}$. Field expressions for the axial fields with non-azimuthal variation $\left(\frac{\partial}{\partial \phi} \neq 0\right)$ can be obtained from Maxwell's equation as:

$$
\begin{array}{cc}
E_{z 1}=A_{m} I_{m}(\gamma r) e^{j(\omega t-\beta z)} e^{j m \phi} & (r \leq a) \\
E_{z 2}=B_{m} K_{m}(\gamma r) e^{j(\omega t-\beta z)} e^{j m \phi} & (r \geq a) \\
H_{z 1}=C_{m} I_{m}(\gamma r) e^{j(\omega t-\beta z)} e^{j m \phi} & (r \leq a) \\
H_{z 2}=D_{m} K_{m}(\gamma r) e^{j(\omega t-\beta z)} e^{j m \phi} & (r \geq a)
\end{array}
$$

In (3.6), the subscripts 1 and 2 are used to represent the field quantities in the regions inside and outside the helix, respectively. $I_{m}$ and $K_{m}$ are the $m^{\text {th }}$ order modified Bessel 
functions of first and second kind, respectively. $A_{m}, B_{m}, C_{m}$, and $D_{m}$ are unknown constants in the field expressions.

\section{B. Periodicity}

The circular helix coincides with itself under two transformations. The first one is the axial translation through an axial pitch length $L$, called the axial transformation. The second one is a rotation through an arbitrary angle $\phi(\phi<2 \pi)$, together with an axial translation of $(L \phi / 2 \pi)$, called the differential skew transformation.

Floquet's theorem is used to introduce the effect of periodicity in the field expressions. Floquet's theorem states that "for a given mode of propagation at a given steady-state frequency in a periodic structure, the fields at one cross-section differ from the fields one period away by a complex constant". Floquet's theorem can be satisfied in a periodic structure with axial period $L$ by the following functional dependence of field quantities:

$$
f(z)=\sum_{n=-\infty}^{\infty} e^{-j \beta_{n} z}=\sum_{n=-\infty}^{\infty} e^{-j \beta_{0} z} e^{\left(\frac{-j 2 \pi n z}{L}\right)}
$$

where $n$ is the number of space harmonic; $\beta_{n}$ and $\beta_{0}$ are the phase constants of the $n^{\text {th }}$ and fundamental space harmonics, respectively. The axial dependence and angular dependence can be combined in the field expressions. The combined field expression for the longitudinal electric field in region 1 can be written as:

$$
E_{z 1}=\sum_{m=-\infty}^{\infty} \sum_{n=-\infty}^{\infty} A_{m, n} I_{m}\left(\gamma_{n} r\right) e^{j\left(\omega t-\beta_{n} z\right)} e^{j m \phi} \quad(r \leq a)
$$

where $\gamma_{n}=\sqrt{\beta_{n}^{2}-k_{0}^{2}}$ is the wave number for the $n^{\text {th }}$ space harmonic.

Even though the field expression (3.8) satisfies the axial transformation, it does not satisfy the differential skew transformation. A careful application of Floquet's theorem in (3.8) reveals that the equation can satisfy the differential skew transformation only by putting $m=n$. Thus, the field expression in (3.8) can be rewritten as:

$$
E_{z 1}=\sum_{n=-\infty}^{\infty} A_{n} I_{n}\left(\gamma_{n} r\right) e^{j\left(\omega t-\beta_{n} z\right)} e^{j n \phi} \quad(r \leq a)
$$

\section{Field expressions for the $n^{\text {th }}$ space harmonic}

The field expression (3.9) contains the fields corresponding to $n$ space harmonics. The axial electric field for the $n^{\text {th }}$ space harmonic in region 1 can be written as: 


$$
E_{z 1, n}=A_{n} I_{n}\left(\gamma_{n} r\right) e^{j\left(\omega t-\beta_{n} z\right)} e^{j n \phi} \quad(r \leq a)
$$

were,

$$
E_{z 1}=\sum_{n=-\infty}^{\infty} E_{z 1, n}
$$

Similar to (3.10), the axial electric field and the axial magnetic field for the $n^{\text {th }}$ space harmonic for the $n^{\text {th }}$ space harmonic in region 2 can be written as:

$$
\begin{array}{ll}
E_{z 2, n}=B_{n} K_{n}\left(\gamma_{n} r\right) e^{j\left(\omega t-\beta_{n} z\right)} e^{j n \phi} & (r \geq a) \\
H_{z 1, n}=C_{n} I_{n}\left(\gamma_{n} r\right) e^{j\left(\omega t-\beta_{n} z\right)} e^{j n \phi} & (r \leq a) \\
H_{z 2, n}=D_{n} K_{n}\left(\gamma_{n} r\right) e^{j\left(\omega t-\beta_{n} z\right)} e^{j n \phi} & (r \geq a)
\end{array}
$$

\section{Boundary conditions}

The unknown field constants $A_{n}, B_{n}, C_{n}$, and $D_{n}$ in the above field expressions can be obtained using the following four boundary conditions at $r=a$ :

$$
\begin{gathered}
E_{z 1, n}=E_{z 2, n} \\
E_{\phi 1, n}=E_{\phi 2, n} \\
H_{\phi 2, n}-H_{\phi 1, n}=J_{z, n} \\
H_{z 1, n}-H_{z 2, n}=J_{\phi, n}
\end{gathered}
$$

where $J_{z, n}$ and $J_{\phi, n}$ are the $n^{\text {th }}$ space harmonic components of the axial and the azimuthal components of surface current densities, respectively.

\section{E. Assumption for current}

The tape-helix analysis assumes that the tape-surface current exists only in the direction parallel to the winding. $J_{z, n}$ and $J_{\phi, n}$ are related to the $n^{\text {th }}$ space harmonic component of the surface current parallel to the winding $\left(J_{\| n}\right)$ as:

$$
\begin{gathered}
J_{z, n}=J_{\| n} \sin \psi \\
J_{\phi, n}=J_{\| n} \cos \psi
\end{gathered}
$$

where $\psi$ is the pitch angle obtained as $\psi=\tan ^{-1}\left(\frac{L}{2 \pi a}\right)$. The total surface current parallel to the winding, $T$, is a sum of $J_{\| n}$ due to all space harmonics and is given as:

$$
T=\sum_{n=-\infty}^{\infty} J_{\| n}
$$


In tape-helix analysis, the total surface current parallel to the winding, $T$, is assumed to have a constant magnitude $J$ and flowing in the direction of the winding. Moreover, $T$ is also assumed to have a phase variation only according to the fundamental space harmonic. Thus, $T$ is given as:

$$
T=\left\{\begin{array}{lc}
J e^{-j \beta_{0} z} & \text { on the tape } \\
0 & \text { for the remaining region }
\end{array}\right.
$$

$J_{\| n}$ can be obtained by considering both equations (3.15) and (3.16) to determine the Fourier series components of $T$ as:

$$
J_{\| n}=J \frac{\sin \left(\frac{\beta_{n} \delta}{2}\right)}{\left(\frac{\beta_{n} \delta}{2}\right)}\left(\frac{\delta}{L}\right)
$$

\section{F. Characteristic equation}

The characteristic equation is obtained by equating the electric field intensity inside the helix parallel to the winding at $r=a$ to zero:

$$
E_{1, \|}=\sum_{n=-\infty}^{\infty} E_{\phi 1, n} \cos \psi+E_{z 1, n} \sin \psi=0
$$

Finally, the characteristic equation is obtained as:

$$
\sum_{n=-\infty}^{+\infty}\left[\begin{array}{c}
\left(\beta_{0}^{2} a^{2}-k_{0}^{2} a^{2}+\frac{k_{0}^{2} a^{2} n^{2} \cot ^{2} \psi}{\left(\beta_{0} a+n \cot \psi\right)^{2}-k_{0}^{2} a^{2}}\right) I_{n}\left(\gamma_{n} a\right) K_{n}\left(\gamma_{n} a\right)+ \\
k_{0}^{2} a^{2} \cot ^{2} \psi I_{n}^{\prime}{ }_{n}\left(\gamma_{n} a\right) K_{n}^{\prime}\left(\gamma_{n} a\right)
\end{array}\right] J\left(\frac{\sin \left(\beta_{n} \delta / 2\right)}{\beta_{n} \delta / 2}\right)=0
$$

where $I_{n}^{\prime}$ and $K_{n}^{\prime}$ are the derivatives of the $n^{\text {th }}$ order modified Bessel functions of the first and second kind, respectively.

One can numerically solve (3.19) to obtain the phase constant of the fundamental space harmonic and that result in turn gives the phase constant of non-fundamental space harmonics. Subsequently one can obtain the field distribution and the interaction impedance of the fundamental as well as the non-fundamental space harmonics from this analysis.

\subsubsection{Measurement}

Resonant perturbation method and non-resonant perturbation method are the commonly used cold-test measurement techniques for the interaction impedance of a SWS. In the resonant perturbation method, the SWS is converted into a resonant circuit using perfect short circuit at both ends of the structure. A dielectric cylinder is inserted along the 
axis of the resonant structure and the resulting change in the resonant frequency is measured [44]. This technique assumes that the dominant field in the region of perturbation is the longitudinal electric field of the fundamental space harmonic. If this assumption is valid, then the measured change in the resonant frequency can be expressed as a function of the dimensions and the permittivity of the dielectric cylinder, unperturbed resonant frequency, group velocity, and the ratio between the longitudinal electric field and the time averaged RF power. Eventually the ratio between the longitudinal electric field and the time averaged RF power can be extracted from the measured results to obtain the interaction impedance. This technique can only measure the interaction impedance of the fundamental space harmonic since the non-fundamental space harmonics do not satisfy the assumptions.

Non-resonant perturbation technique is more suitable for measuring the interaction impedance of the SWSs which cannot be perfectly shorted. The mathematical expressions for this technique are derived from the resonant perturbation technique. Both techniques use quite similar assumptions. This technique measures the change in the phase constant due to the insertion of a dielectric cylinder into the SWS for determining the interaction impedance of the fundamental space harmonic [46], [47]. This technique also fails in measuring the interaction impedance of non-fundamental space harmonics.

\subsubsection{Simulation}

EM simulation is the simplest and easiest method to determine the interaction impedance of a SWS. Different simulation tools like Computer Simulation Technology (CST) Microwave Studio (MWS), Ansys High Frequency Structure Simulator (HFSS), MAGIC, etc are used for finding the interaction impedance of SWSs. The phase constant and the field distribution in a SWS are obtained from the simulation tools by simulating a single period of the structure.

Simulation has been used for finding the interaction impedance of circular helix SWS by Kory and Dayton [95]. The results from 3-D simulation code MAFIA have been used for determining the interaction impedance of fundamental space harmonic in the SWS. Kory and Dayton have used Fourier decomposition method to extract the magnitude of longitudinal electric field for fundamental space harmonic from the total longitudinal electric field of the SWS. The results from this work concluded that the interaction impedance obtained from the simulation has more accuracy than that obtained from the measurement. 
The emphasis in [95] was on the on-axis values of the interaction impedance for the fundamental space harmonic. The values of interaction impedance for off-axis positions and the values for the non-fundamental space harmonics were not reported. Nowadays, simulation tools are popularly used for determining the interaction impedance for the fundamental [96] as well as the non-fundamental space harmonics [72]. But in almost no case it is mentioned whether the interaction impedance results are obtained using the Fourier decomposition method proposed in [95]. Here we consider the interaction impedance for various space harmonics of circular helix SWS based on simulations using CST Microwave Studio. The accuracy of the simulation results is assessed by comparing these with the results obtained from the tape-helix analysis.

\subsection{Comparison Between Tape-Helix Analysis and Simulation}

In this section, the phase constant and the magnitude of longitudinal electric field from simulation and the tape-helix analysis are compared for different space harmonics. The results from comparison are used for assessing the accuracy of the simulation results. The measured results are not used for the comparison since such results for the nonfundamental space harmonics are unavailable. Besides, the comparison between simulated and measured results for the fundamental space harmonic has already been reported in [95].

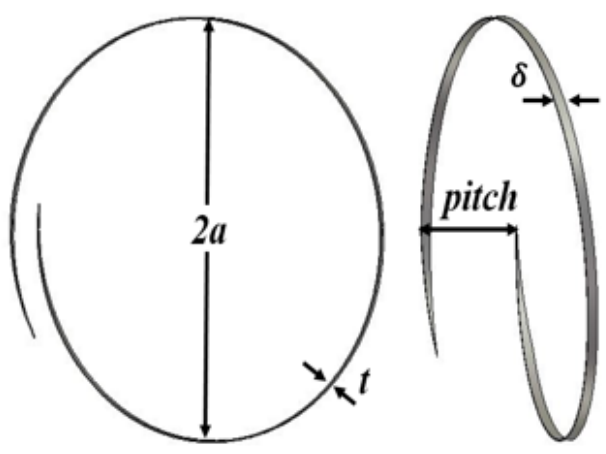

\begin{tabular}{|c|c|}
\hline Parameter & Value (mm) \\
\hline $\mathrm{a}$ & 1.45 \\
\hline Pitch & 0.8 \\
\hline$\delta$ & 0.08 \\
\hline $\mathrm{t}$ & 0.01 \\
\hline
\end{tabular}

Figure 3.3: Circular tape-helix with dimensions.

As shown in Fig. 3.3, a circular helix with inner radius $a=1.41 \mathrm{~mm}$, and pitch length $L=0.8 \mathrm{~mm}$ is used for the simulations. To closely match the simplifying assumptions of very thin and narrow tape made in the tape-helix analysis, tape thickness $t$ and tape width $\delta$ of the circular helix is selected as $0.01 \mathrm{~mm}$ and $0.08 \mathrm{~mm}$, respectively. The circular helix is considered to be lossless and immersed in free space. The simulation and analysis results provided in this section uses the circular tape-helix with dimensions shown in Fig. 3.3. 
The steps for obtaining the magnitude of longitudinal electric field for different space harmonics from simulation are presented in sub-section 3.3.1. This section also discusses the convergence of the results obtained from simulation. The steps for obtaining the magnitude of longitudinal electric field for different space harmonics from analysis are elaborated in sub-section 3.3.2. Section 3.3.3 presents the comparison of phase constant and the magnitude of longitudinal electric field from simulation and the tape-helix analysis. Finally, the reason for mismatch in the comparison of magnitude of longitudinal electric field for the non-fundamental space harmonics is discussed in section 3.3.4

\subsubsection{Magnitude of longitudinal electric field for different space harmonics from} simulation

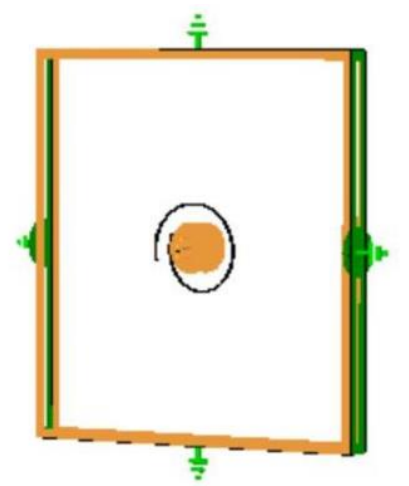

Figure 3.4:.3D model of the tape-helix in Computer Simulation Technology (CST) eigenmode solver

The steps for obtaining the magnitude of longitudinal electric field for different space harmonics are described in detail as follows:

a) The simulations use Eigenmode solver in the CST Microwave Studio. This solver is a frequency domain solver. As shown in Fig. 3.3, the 3D geometry of one-unit cell (one period) of the tape-helix is drawn in the Eigenmode solver. Periodic boundary conditions are applied at both longitudinal boundaries of the unit cell. Fig. 3.4 shows that perfect electric boundaries constitute the transverse boundaries of the unit cell. (The 2016/2017 version of CST Eigenmode solver available to us does not support open boundaries. The effect of separation between the PEC boundary and the SWS is described in section 3.3.1.3). Phase of the EM signal applied to the input of the unit cell is tuned from $0^{\circ}$ to $180^{\circ}$. The simulator gives the eigenfrequency of the SWS corresponding to each phase value. The phase value 
serves as the phase constant for the fundamental space harmonic $\left(\beta_{0}\right)$ for this frequency.

b) The 3D plot of the total longitudinal electric field $E_{z}(r, \phi, z)$ for each of the abovementioned eigenfrequency is also obtained as a result in step (a). From this 3D plot, we can obtain the complex values of $E_{z}(r, \phi, z)$ for a particular value of $(r, \phi)$ in the SWS using the post processing step in CST. The obtained values are the complex values of total longitudinal electric field $E_{z}(r, \phi, z)$ as a function of $z$.

c) The total longitudinal electric field $E_{z}(r, \phi, z)$ obtained from the simulator can be expressed as:

$$
E_{z}(r, \phi, z)=\sum_{n=-\infty}^{\infty}\left|E_{z, n}(r, \phi)\right| e^{-j \beta_{n} z}
$$

d) The total longitudinal electric field for a particular value of $(r, \phi)$ and frequency is used for finding the value of the magnitude of longitudinal electric field for the $n^{\text {th }}$ space harmonic $\left(\left|E_{z, n}(r, \phi)\right|\right)$ by using the following equation:

$$
\left|E_{z, n}(r, \phi)\right|=\frac{1}{p i t c h} \int_{0}^{p i t c h} E_{z}(r, \phi, z) e^{j \beta_{n} z} d z
$$

e) As we run the Eigenmode solver for the complete range of phase values $\left(0^{\circ}\right.$ to $\left.180^{\circ}\right)$, we get the values of total $E_{z}(r, \phi, z)$ and $\beta_{0}$ for the corresponding range of frequencies. From $\beta_{0}$, we can simply calculate the phase constant for the $n^{\text {th }}$ space harmonic $\left(\beta_{n}\right)$.

f) By repeating steps (a)-(d) for different frequencies and different space harmonics, we get the value of the magnitude of longitudinal electric field $\left(\left|E_{z, n}(r, \phi)\right|\right)$ for different space harmonics over the complete range of frequencies.

The integration in (3.21) can be carried out in MATLAB or by using the postprocessing in CST. We verified that the results from both methods are identical. We used MATLAB programme with command "trapz" for carrying out the integration. 


\subsubsection{CONVERGENCE WITH RESPECT TO NUMBER OF MESH CELLS}

The convergence with respect to the number of mesh cells for obtaining the total longitudinal electric field $E_{z}(r, \phi, z)$ as a function of $\mathrm{z}$ as mentioned in step (b) in above section is shown here. With reference to Fig. 3.3, we choose a datapoint at every 0.0005 $\mathrm{mm}$ in the helix for the results in this section. So, we get 1600 data points over one period (values of $E_{z}(r, \phi, z)$ ) as a function of $\mathrm{z}$. We also choose $r=0, \phi=0$ and frequency of $8.32 \mathrm{GHz}$. This frequency corresponds to a phase value of $90^{\circ}$. The effect of the number of data points has been described in section 3.3.12.

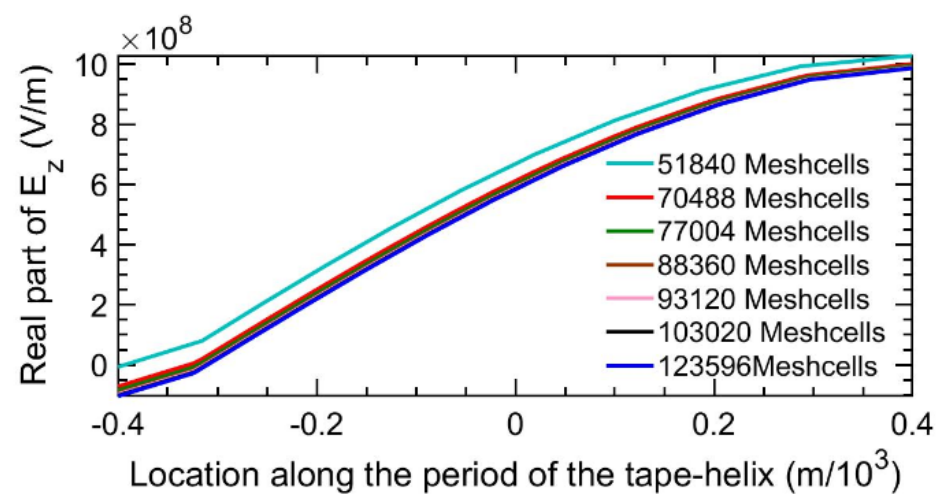

Figure 3.5: Real part of $E_{z}(r=0, \phi=0, z)$ for $8.32 \mathrm{GHz}$.

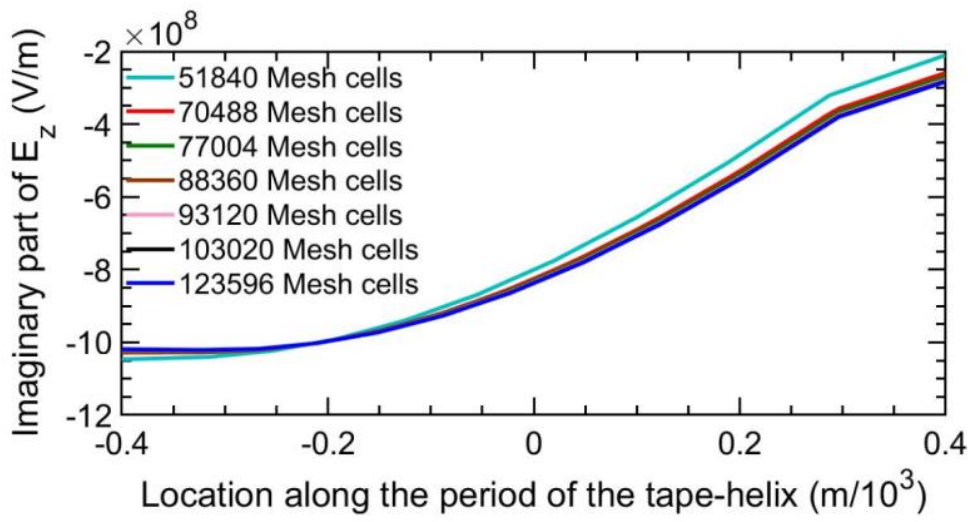

Figure 3.6: Imaginary part of $E_{z}(r=0, \phi=0, z)$ for $8.32 \mathrm{GHz}$.

The complex (real and imaginary) spatial data for the total longitudinal electric field with different mesh size are shown in Figs. 3.5-3.6, respectively. We use tetrahedral mesh with number of mesh cells increasing from 51840 to 123596 over one period of the tapehelix. Simplified versions of Fig. 3.5, and Fig. 3.6, which contain only the complex spatial data for the total longitudinal electric field with103020, and 123596 mesh cells, are shown in Fig. 3.7, and Fig. 3.8, respectively. Fig. 3.7, and Fig. 3.8 clearly show the convergence of the total longitudinal electric field with mesh size. The maximum difference in the values 
of the real part of $E_{z}$ for 123596 and 103020 mesh cells is $0.1 \%$. Similarly, the maximum difference in the imaginary part of $E_{z}$ for 123596 and 103020 mesh cells is $0.6 \%$.

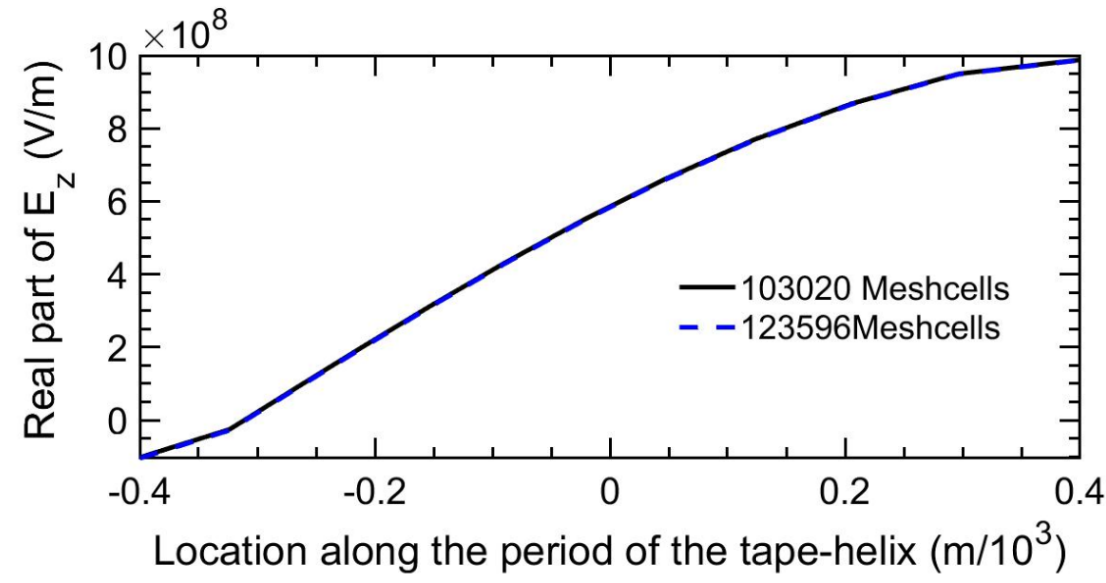

Figure 3.7: Real part of $E_{z}(r=0, \phi=0, z)$ for $8.32 \mathrm{GHz}$.

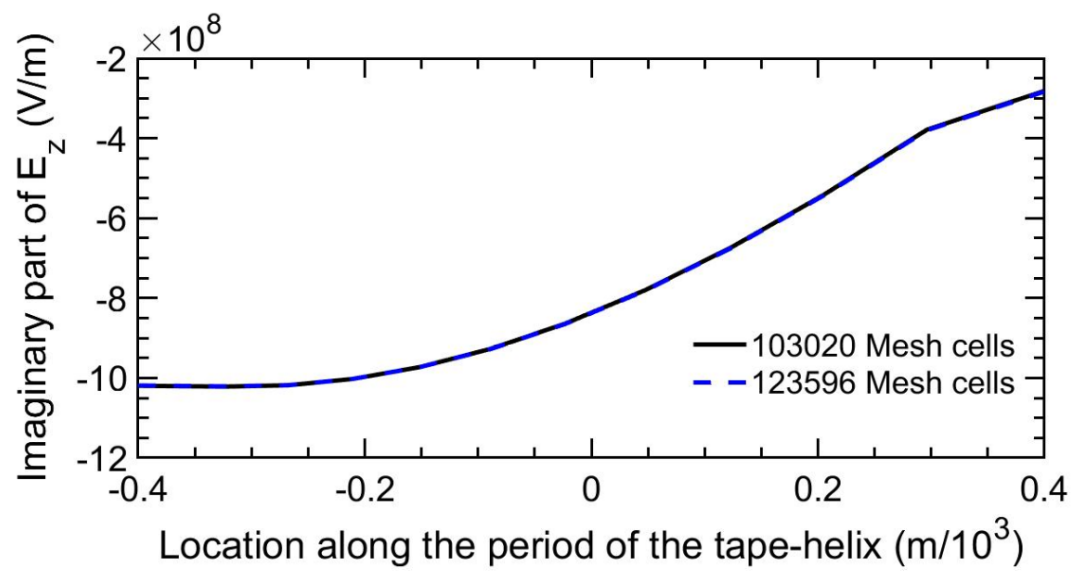

Figure 3.8: Imaginary part of $E_{z}(r=0, \phi=0, z)$ for $8.32 \mathrm{GHz}$.

The Fourier coefficients for various space harmonics can be obtained by substituting the complex data of $E_{z}(r=0, \phi=0, z)$ in (3.20). The MATLAB programme for calculating the Fourier coefficients for different space harmonics is given as Appendix B. Fig. 3.9 shows the variation of the Fourier coefficient for the fundamental space harmonic with number of mesh cells. The difference in the Fourier coefficient values for the fundamental space harmonic for 123596 and 103020 mesh cells is $0.05 \%$.

The variation of the Fourier coefficients for the $n=-1,+1,-2$ and +2 space harmonics with number of mesh cells is shown as Figs. 3.10-3.13, respectively. Figs. 3.9-3.13 show that the values of the Fourier coefficients for all space harmonics converge with the increase in the number of mesh cells. 


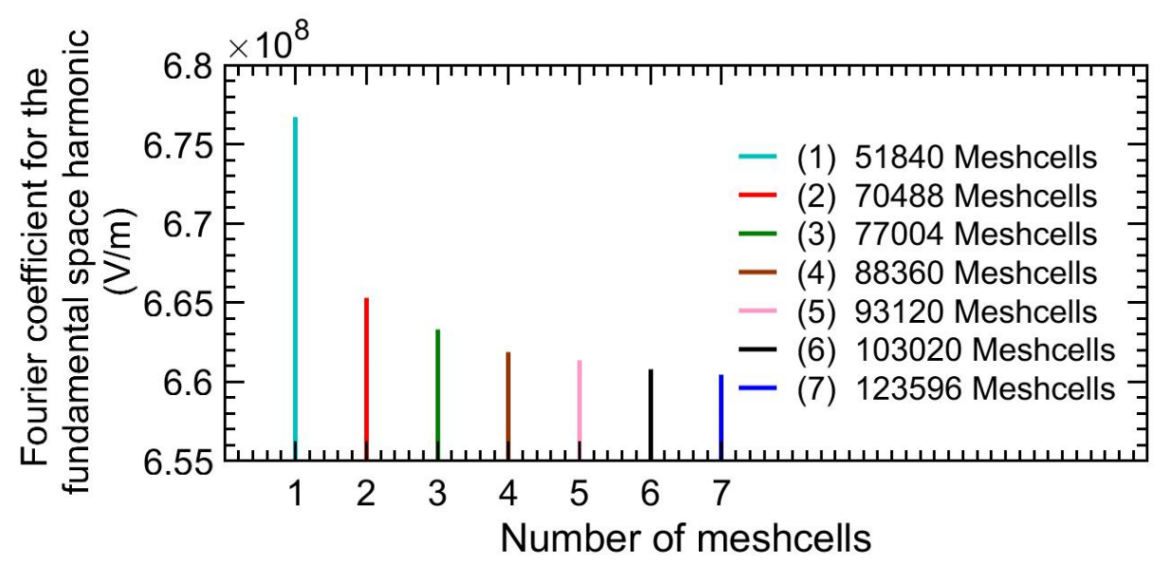

Figure 3.9: Variation of the Fourier coefficient for the fundamental space harmonic with number of mesh cells for $r=0, \phi=0$ and frequency of $8.32 \mathrm{GHz}$.

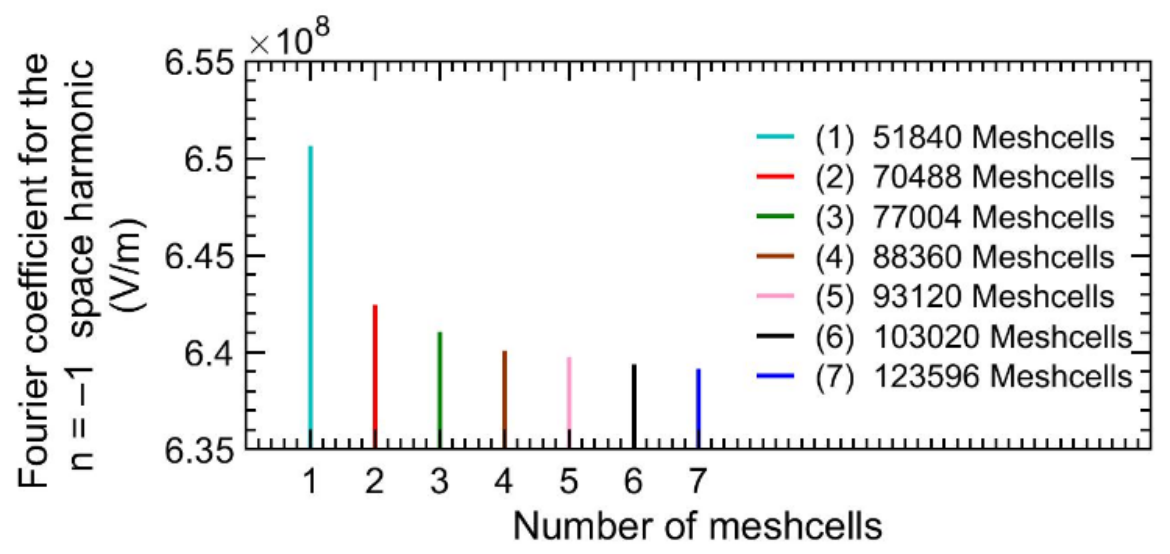

Figure 3.10: Variation of the Fourier coefficient for the $n=-1$ space harmonic with number of mesh cells for $r=0, \phi=0$ and frequency of $8.32 \mathrm{GHz}$.

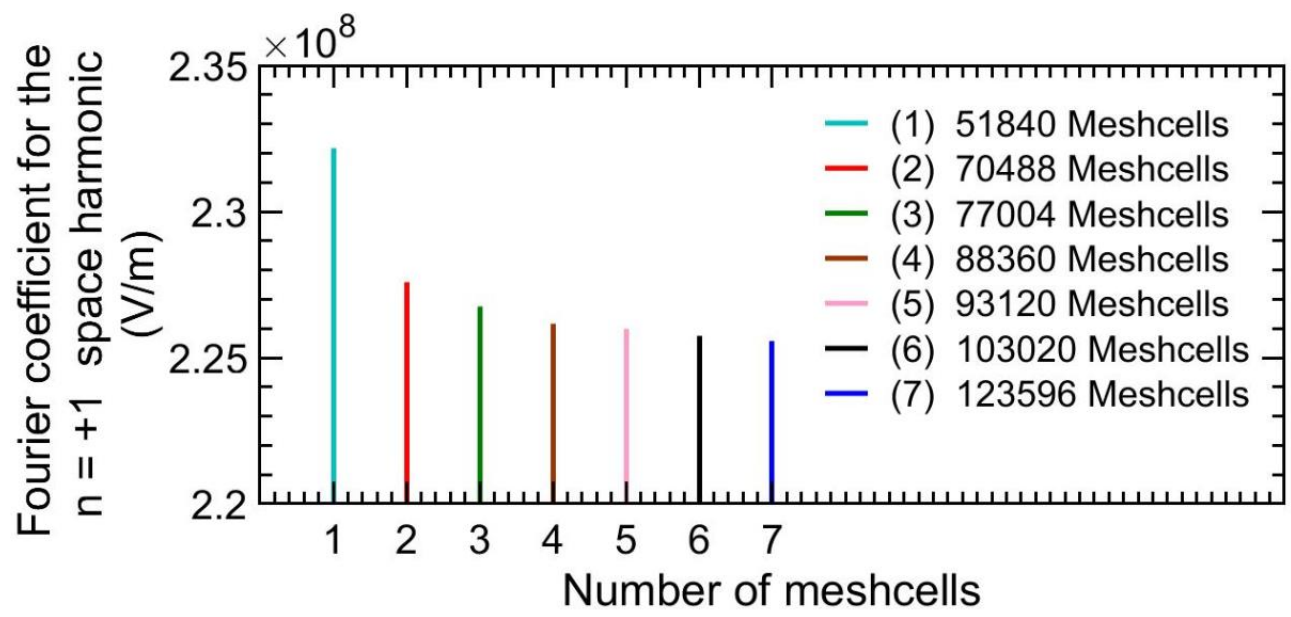

Figure 3.11: Variation of the Fourier coefficient for the $n=+1$ space harmonic with number of mesh cells for $r=0, \phi=0$ and frequency of $8.32 \mathrm{GHz}$. 


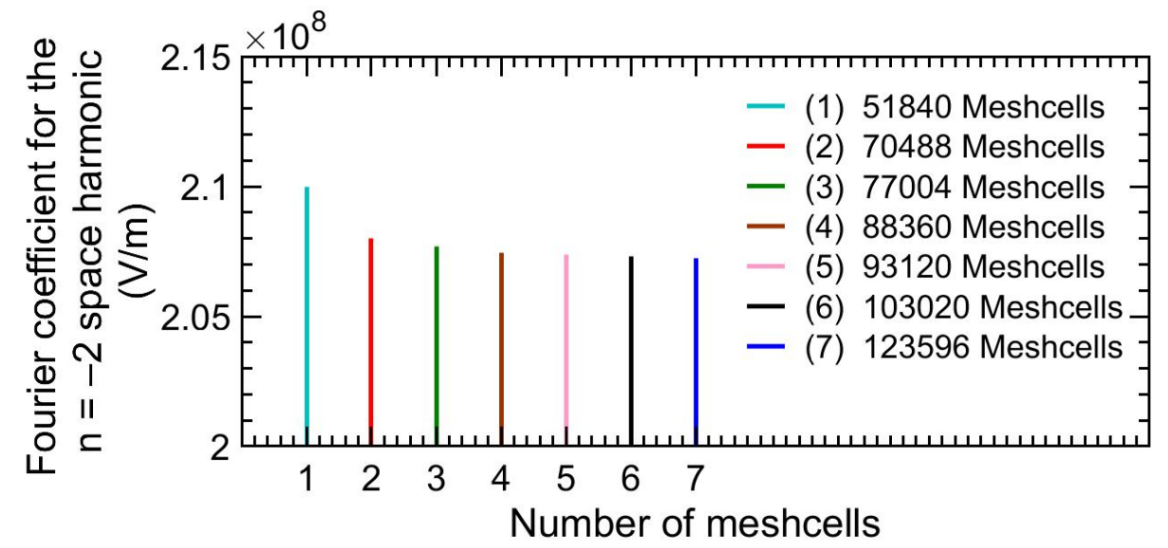

Figure 3.12: Variation of the Fourier coefficient for the $n=-2$ space harmonic with number of mesh cells for $r=0, \phi=0$ and frequency of $8.32 \mathrm{GHz}$.

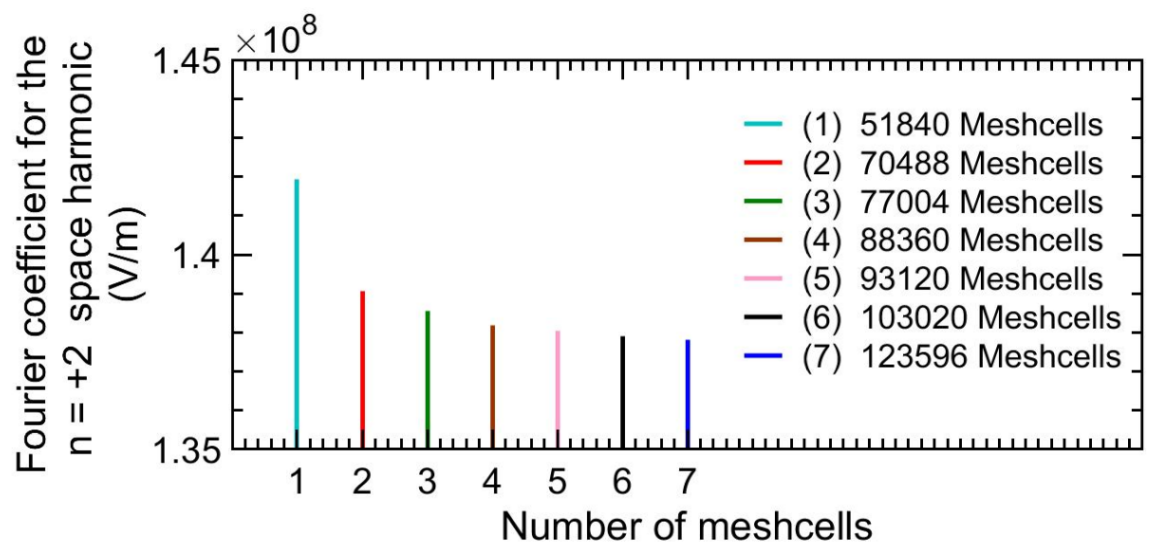

Figure 3.13: Variation of the Fourier coefficient for the $n=+2$ space harmonic with number of mesh cells for $r=0, \phi=0$ and frequency of $8.32 \mathrm{GHz}$.

One can conclude that 123596 tetrahedral mesh cells yield a converged result for the total longitudinal electric field as well as the Fourier coefficients for various space harmonics. This is the number of mesh cells used for the results reported in this chapter.

\subsubsection{CONVERGENCE WITH RESPECT TO DATA POINTS USED FOR INTEGRATION}

Fig. 3.14 shows the variation of the Fourier coefficient for the fundamental space harmonic with number of data points over one period. The difference in the Fourier coefficient values for the fundamental space harmonic for 800 data points and 1600 data points is $0.01 \%$. The variation of the Fourier coefficients for the $n=-1$ and +1 space harmonic with number of data points is shown in Figs. 3.15-3.16, respectively. These figures show that the values of the Fourier coefficients for various space harmonics converge with increase in the number of data points. 


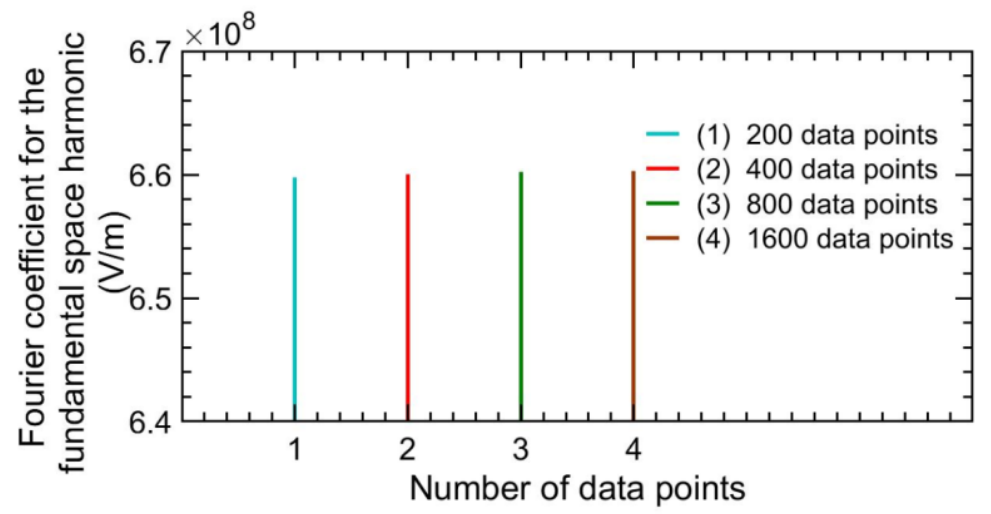

Figure 3.14: Variation of the Fourier coefficient for the fundamental space harmonic with number of mesh data points for $r=0, \phi=0$ and frequency of $8.32 \mathrm{GHz}$.

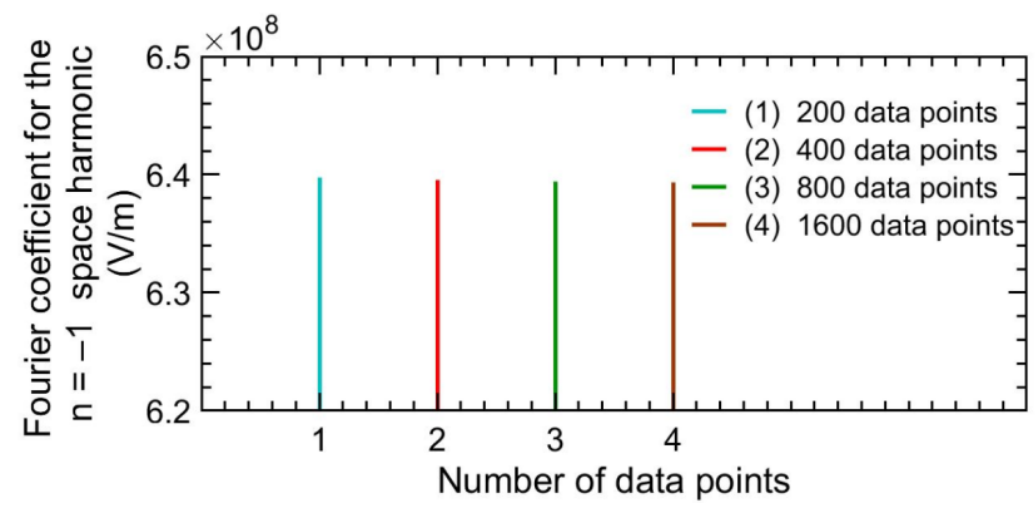

Figure 3.15: Variation of the Fourier coefficient for the $n=-1$ space harmonic with number of mesh data points for $r=0, \phi=0$ and frequency of $8.32 \mathrm{GHz}$.

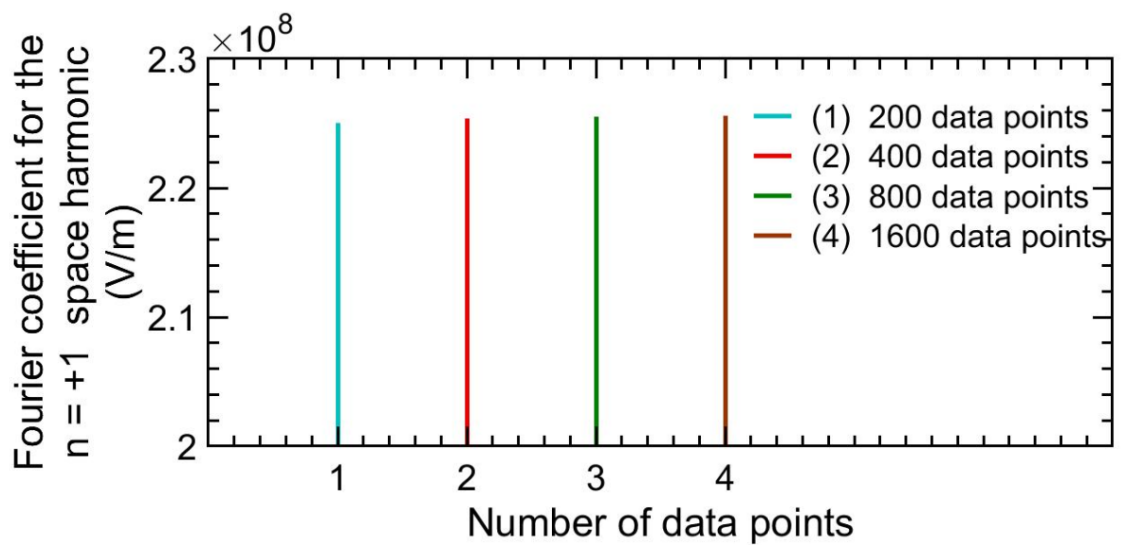

Figure 3.16: Variation of the Fourier coefficient for the $n=+1$ space harmonic with number of mesh data points for $r=0, \phi=0$ and frequency of $8.32 \mathrm{GHz}$.

One can conclude that 1600 data points yield a converged result for the Fourier coefficients for various space harmonics. This is the number of data points used for the results reported in this chapter. 
3.3.1.3 CONVERGENCE WITH RESPECT TO SEPARATION OF THE PEC BOUNDARY FROM THE STRUCTURE

In this and the next chapter, we have simulated the SWSs in free space using Eigenmode solver. We have used PEC boundary instead of open boundary in the transverse directions of the SWS because the Eigenmode solver available to us (version 2016 and 2017) does not support open boundary. We keep the PEC boundary at a sufficiently large separation (a separation of 3.5 times the wavelength at midfrequency $7.5 \mathrm{GHz}$ ) from the SWS to minimize the effect of the boundary. Usually such results are considered adequate for a SWS in free space.

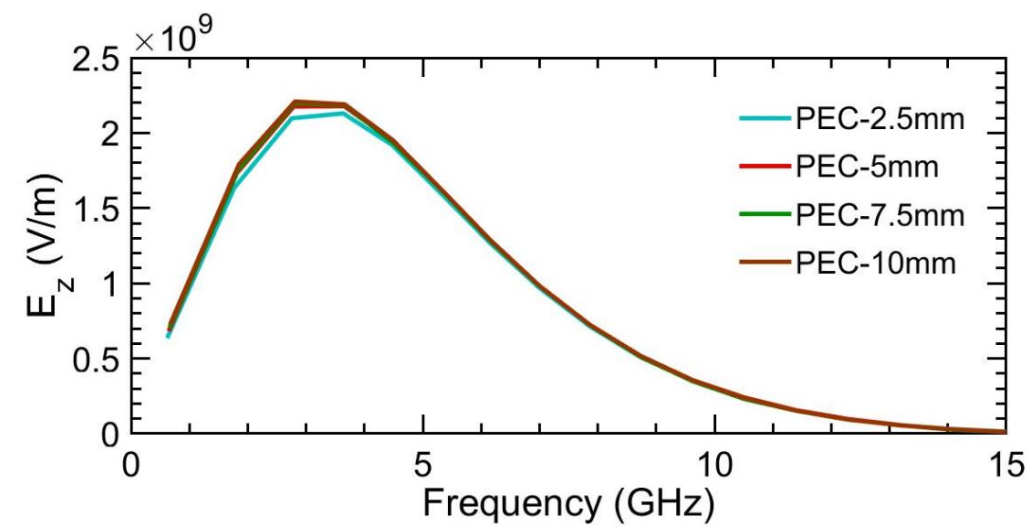

Figure 3.17: Variation of magnitude of Ez vs. frequency for the fundamental space harmonic at axis of the helix with different separations of the PEC boundary from the circular tape-helix.

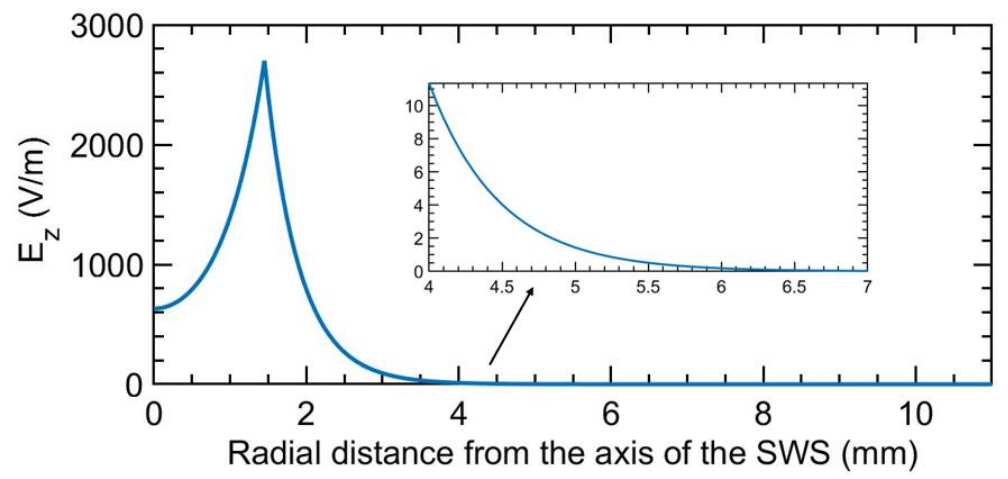

Figure 3.18: Variation of magnitude of Ez vs. radial distance from the axis of the SWS for the fundamental space harmonic from the circular tape-helix.

Fig. 3.17 presents the results of magnitude of $E_{z}$ versus frequency for the circular tape-helix in Fig. 3.3 for the fundamental space harmonic with different separations of the 
PEC boundary. As shown, the values of magnitude of $E_{z}$ increase slightly as the PEC boundary moves away from the SWS. Maximum difference in the magnitude of $E_{z}$ with PEC boundary at $7.5 \mathrm{~mm}$ and $10 \mathrm{~mm}$ from the SWS is $0.08 \%$. Thus, we get a converged result for a separation of $10 \mathrm{~mm}$ between the SWS and the PEC boundary.

Fig. 3.18 shows the variation of magnitude of $E_{z}$ for the fundamental space harmonic with radial distance from the axis of the SWS. This result is obtained from the field expressions of circular tape-helix (Explained in the sub section 3.2.1). As shown, the values of magnitude of $E_{z}$ increases when one moves from the axis to the circular tapehelix. Whereas the value of magnitude of $E_{z}$ decreases exponentially when one moves away from the circular tape-helix. Fig. 3.18 show the magnitude of $E_{z}$ is negligibly small at a large distance from the SWS. Hence the simulation results with PEC boundary at a large enough distance (a distance of 3.5 times the wavelength at midfrequency $7.5 \mathrm{GHz}$ ) can be closely approximated to the simulation results with open boundary.

As regards the simulation results, section 3.3.1 explains in a step-by-step fashion how to get the magnitude of longitudinal electric field $\left(\left|E_{z, n}(r, \phi)\right|\right)$ at a specific value of $r$ and $\phi$ in the SWS for different space harmonics as a function of frequency. Sections 3.3.1.1 describes that 123596 tetrahedral mesh cells provide converged results for eigenmode simulation and section 3.3.1.2 describes that 1600 number of data points provide converged results for integration (3.21). Section 3.3.1.3 describes that the separation of $10 \mathrm{~mm}$ between the PEC boundary and the structure leads to converged results.

\subsubsection{Magnitude of longitudinal electric field for different space harmonics from} analysis

The following steps explain how we get the analytical results for the magnitude of longitudinal electric field $\left(\left|E_{z, n}(r, \phi)\right|\right)$ at a specific value of $r$ and $\phi$ for comparison with simulation results.

a) The phase constant for the fundamental space harmonic $\beta_{0}$ for the circular tapehelix is obtained by solving the transcendental characteristics equation:

$$
\sum_{n=-\infty}^{+\infty}\left[\begin{array}{c}
\left(\beta_{0}^{2} a^{2}-k_{0}^{2} a^{2}+\frac{k_{0}^{2} a^{2} n^{2} \cot ^{2} \psi}{\left(\beta_{0} a+n \cot \psi\right)^{2}-k_{0}^{2} a^{2}}\right) I_{n}\left(\gamma_{n} a\right) K_{n}\left(\gamma_{n} a\right)+ \\
k_{0}^{2} a^{2} \cot ^{2} \psi I^{\prime}{ }_{n}\left(\gamma_{n} a\right) K^{\prime}{ }_{n}\left(\gamma_{n} a\right)
\end{array}\right] J\left(\frac{\sin \left(\beta_{n} \delta / 2\right)}{\beta_{n} \delta / 2}\right)=0
$$


b) The solution of (3.19) gives the value of $\beta_{0}$ for different frequencies.

c) The phase constant for the $n^{\text {th }}$ space harmonic $\beta_{n}$ is calculated from $\beta_{0}$.

d) The magnitude of the longitudinal electric field $\left|E_{z 1, n}\right|$ in Region 1 (Fig. 3.1 (c)) is calculated by substituting the value of $\beta_{n}$ and $(r, \phi)$ in (3.22):

$$
\left|E_{z 1, n}\right|=A_{n} I_{n}\left(\gamma_{n} r\right) \quad(r \leq a)
$$

e) The only unknown in (3.22) is $A_{n}$, which is obtained by using the following equation:

$$
A_{n}=\frac{\gamma_{n} K_{n}\left(\gamma_{n} a\right)}{j \omega \epsilon_{0}} \frac{\left(\sin \psi-\cos \psi\left(n \beta_{n} / a \gamma_{n}^{2}\right)\right)}{I_{n}\left(\gamma_{n} a\right) K^{\prime}{ }_{n}\left(\gamma_{n} a\right)-I^{\prime}{ }_{n}\left(\gamma_{n} a\right) K_{n}\left(\gamma_{n} a\right)}
$$

f) In this manner one can plot $\left|E_{z 1, n}\right|$ versus frequency for different space harmonics.

\subsubsection{Comparison of Results}

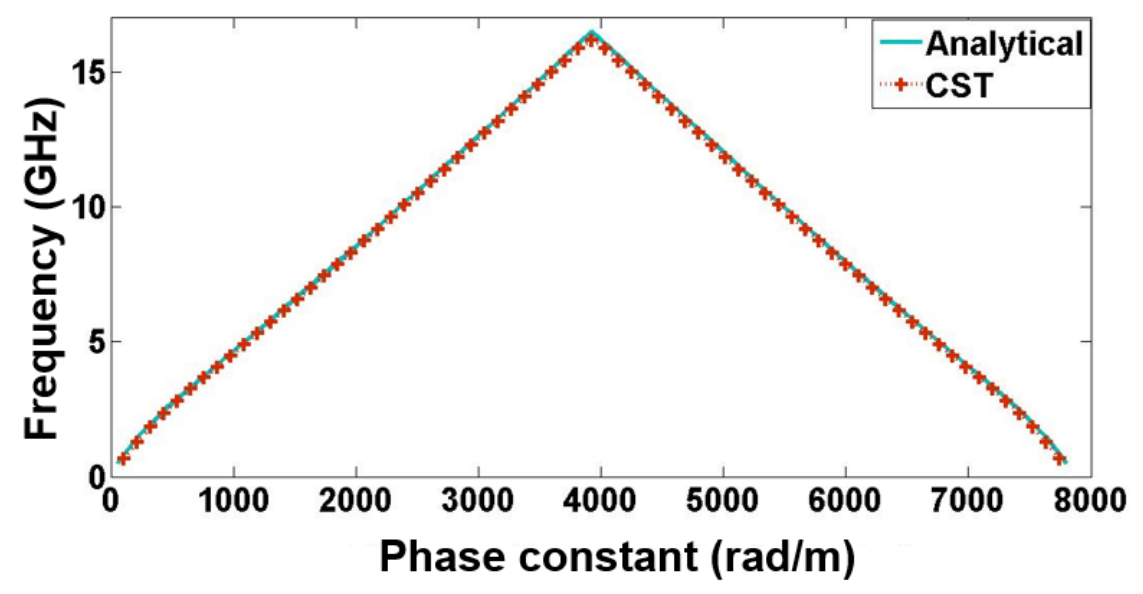

Figure 3.19: Comparison of dispersion characteristics for the circular helix.

The simulated values of the phase constant and the total longitudinal electric field $\left(E_{z}\right)$ in a particular circular helix are obtained using CST microwave studio. The phase constant values of the SWS obtained from the simulations are compared with those from the numerical solution of the characteristic equation of the tape-helix analysis, i.e., (3.19). Even though (3.19) contains infinite number of space harmonics, considering the first seven space harmonics gives a converged result. As shown in Fig. 3.19, the dispersion 
characteristics of the circular helix for the fundamental and the $n=-1$ space harmonic obtained from the simulations show a very close match with those from the analysis.

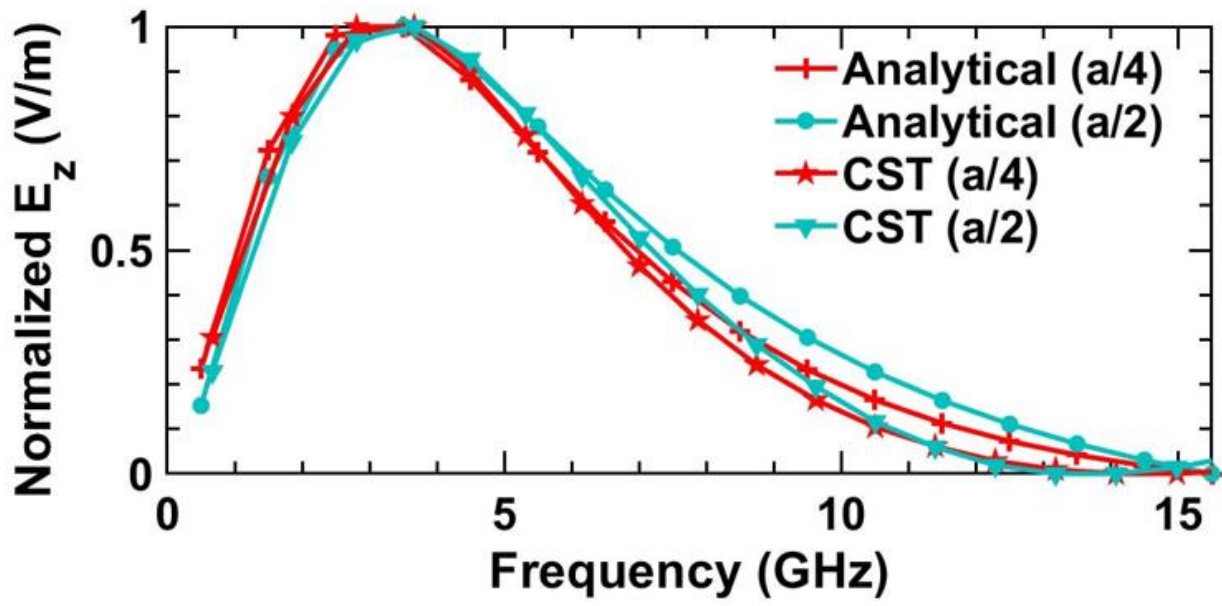

Figure 3.20: Comparison of normalized values of the magnitude of $E_{z}$ for the fundamental space harmonic at two different off-axis positions; $a$ is the inner radius of the helix.

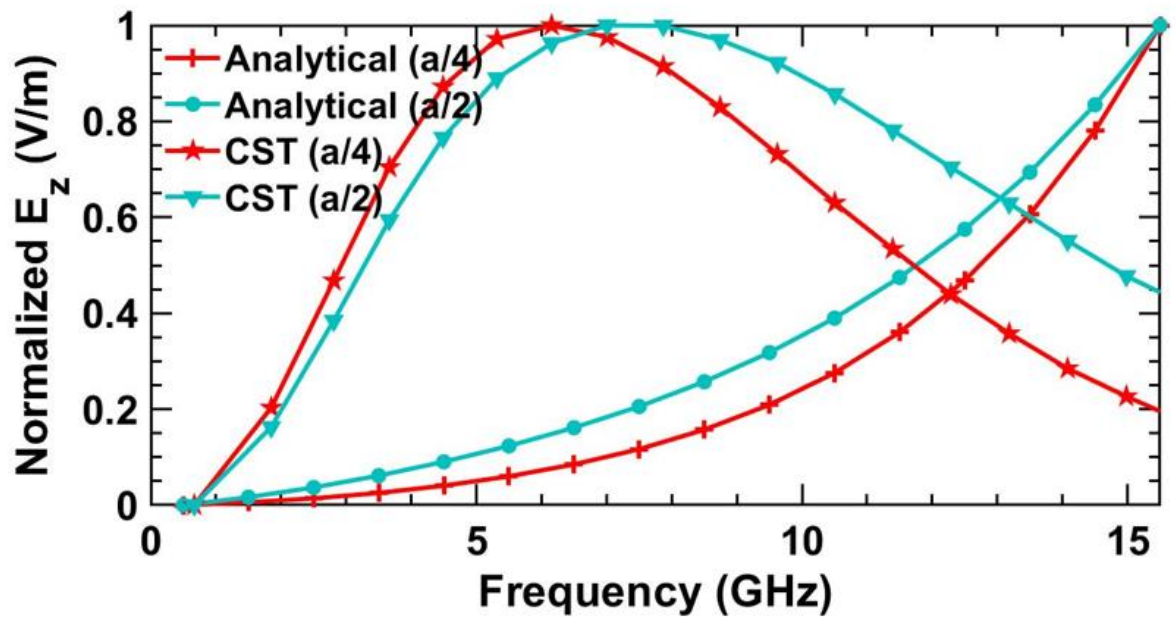

Figure 3.21: Comparison of normalized values of the magnitude of $E_{z}$ for the $\mathrm{n}=-1$ space harmonic at two different off-axis positions; $a$ is the inner radius of the helix.

The total longitudinal electric field for the circular helix SWS shown in Fig. 3.3 at a particular beam position is directly obtained from the simulator. This field is Fourier decomposed using (3.21) to extract the magnitude of longitudinal electric field for a specific space harmonic (discussed in section 3.3.1.1). These values are compared with the magnitude of longitudinal electric field for the specific space harmonic obtained from the tape-helix analysis. Normalized values of the magnitude of longitudinal electric field $\left(E_{z}\right)$ 
vs. frequency for the fundamental space harmonic at two different off-axis positions from the simulation and analysis are shown in Fig. 3.20. As shown in the figure the variation of magnitude of $E_{z}$ for the fundamental space harmonic from simulation and analysis matches quite well in general. But the normalized values of magnitude of $E_{z}$ vs. frequency for the $\mathrm{n}=-1$ space harmonic, shown in Fig. 3.21, do not match at all. In Fig. 3.20 and Fig. 3.21, each curve is normalized with respect to its own maximum value so as to bring out the difference in the nature of variation for the analytical and simulation results clearly. Similarly, the analytical and simulated normalized values of magnitude of $E_{z}$ for all other non-fundamental space harmonics also show a clear mismatch.

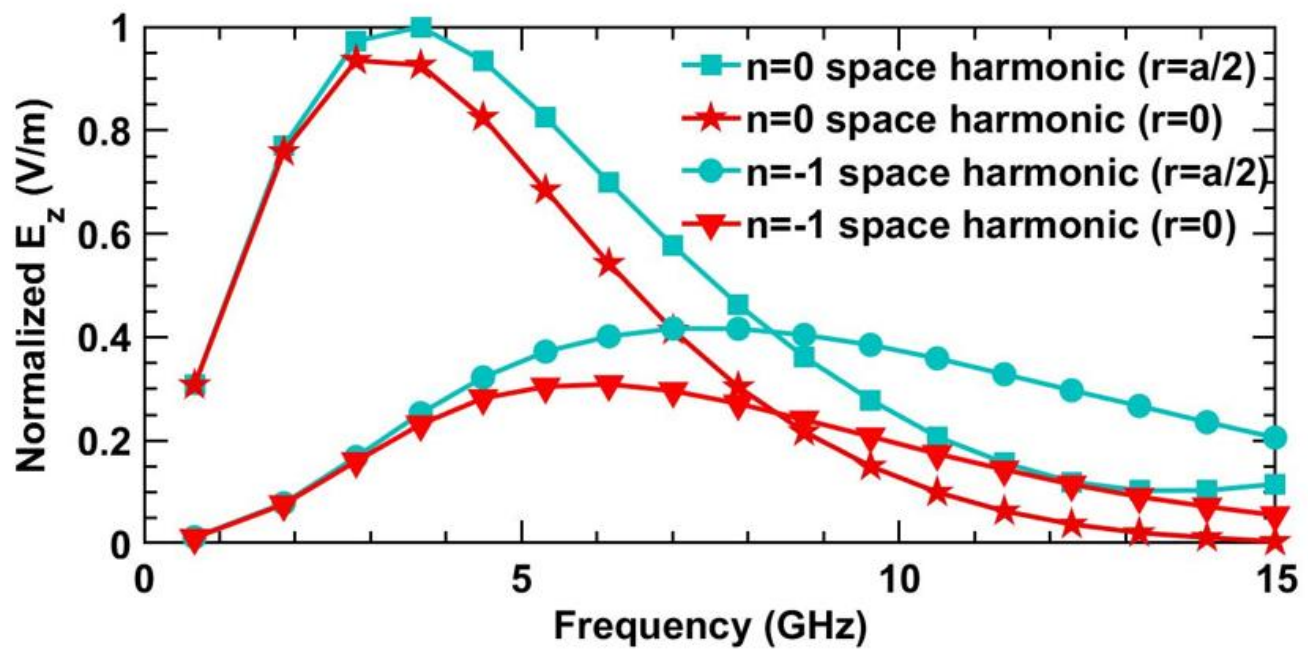

Figure 3.22: Comparison of normalized values of magnitude of $E_{z}$ from simulations for the fundamental and $n=-1$ space harmonic at on-axis and off-axis positions.

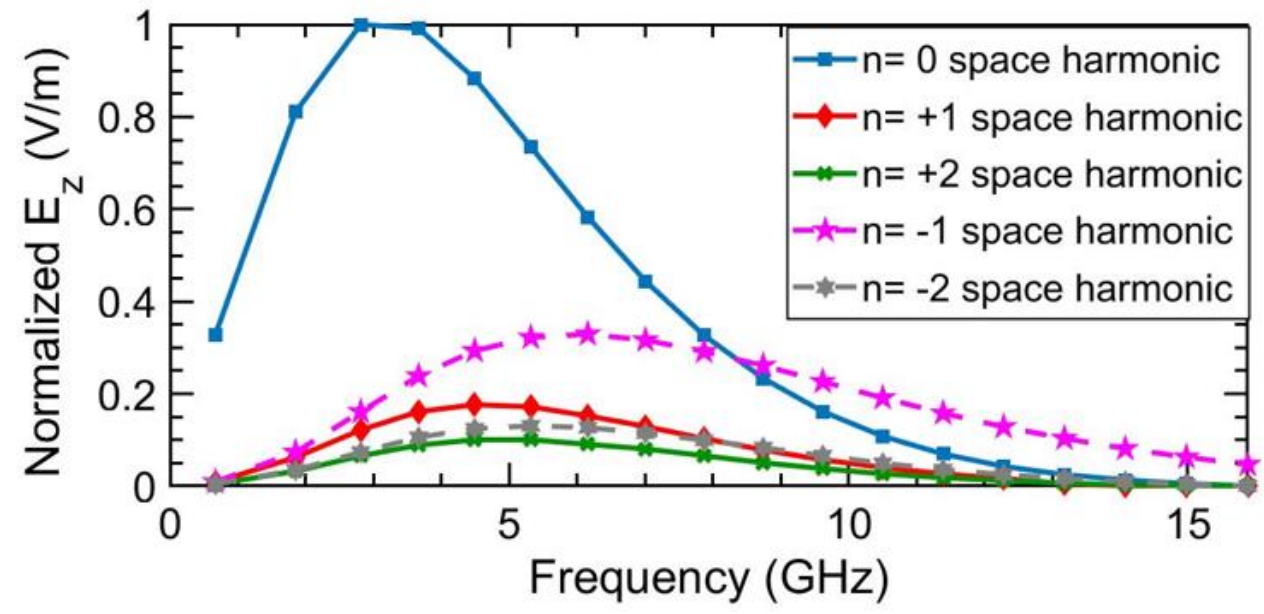

Figure 3.23: Comparison of normalized values of magnitude of $E_{z}$ from simulations for the first five space harmonics at on-axis position. 
The simulated normalized values of $E_{z}$ vs. frequency for the fundamental and the n= -1 space harmonics at two different positions are shown in Fig. 3.22. In this case, the curves are normalized with respect to the maximum value of magnitude of $E_{z}$ for the fundamental space harmonic at $r=a / 2$ so as to show the relative amplitude of the curves. Contrary to common belief, the comparison indicates that the amplitude of the $n=-1$ space harmonic is comparable to that of the fundamental space harmonic. Fig. 3.22 also shows that the onaxis amplitude of $E_{Z}$ of the $\mathrm{n}=-1$ space harmonic has a significant value, whereas the tapehelix analysis predicts it to be zero. These results indicate that either the simulation or the tape-helix analysis fails to provide the correct values of the magnitude of longitudinal electric field for non-fundamental space harmonic. In this context, we revisit the assumptions made in the tape-helix analysis.

The simulated normalized values of on-axis $E_{z}$ vs. frequency for first five space harmonics are shown in Fig. 3.23. Again, the curves are normalized with respect to the maximum value of $E_{z}$ for the fundamental space harmonic. The figure shows that the onaxis amplitude of $E_{z}$ decreases with increase in the order of space harmonic. Fig. 3.23 also shows that the on-axis amplitude of $E_{Z}$ of the $n=-1$ space harmonic has higher vaue than that of the $n=+1$ space harmonic.

\subsubsection{Limitations of Tape-Helix Analysis}

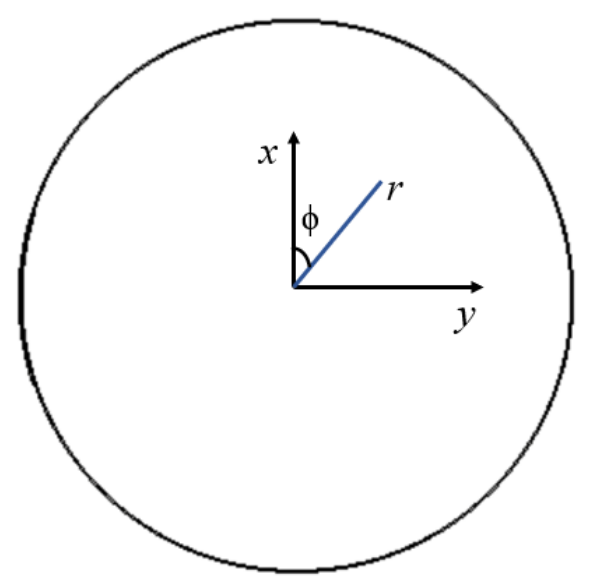

Figure 3.24: The side view of the circular helix with coordinate system. The $\mathrm{z}$ axis is going into the plane of the figure.

The complexity of the tape-helix analysis is reduced by making two major assumptions for the current through the helix. As expressed in (3.16), the first assumption considers that the current along the tape flows only in the direction of winding with a constant magnitude 
along the strip-width. The phase factor of the current is assumed as $e^{-j \beta_{0} Z}$ in the second assumption, i.e., the phase varies only according to the propagation constant of the fundamental space harmonic.

The validity of these assumptions is assessed by checking the values of the magnitude and phase of the total current density along the width of the tape from simulations. These simulation results have been obtained from the CST Eigenmode solver. Details of this type of simulation have been given in section 3.3.1. The simulation model used in the Eigenmode solver is shown in Fig. 3.3. This figure includes the dimensions of the tape-helix. Fig. 3.24 shows the side view of the circular helix.

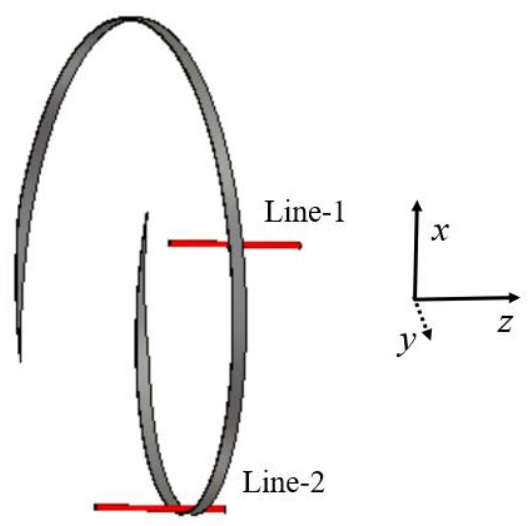

Figure 3.25: The perspective view of the circular tape helix with two lines drawn on the inner surface of the tape, corresponding to $\phi=90^{\circ}$ (line 1) and $\phi=180^{\circ}$ (line 2). The Cartesian coordinate system is also shown; the origin of the coordinate system is at $\mathrm{z}=0$.

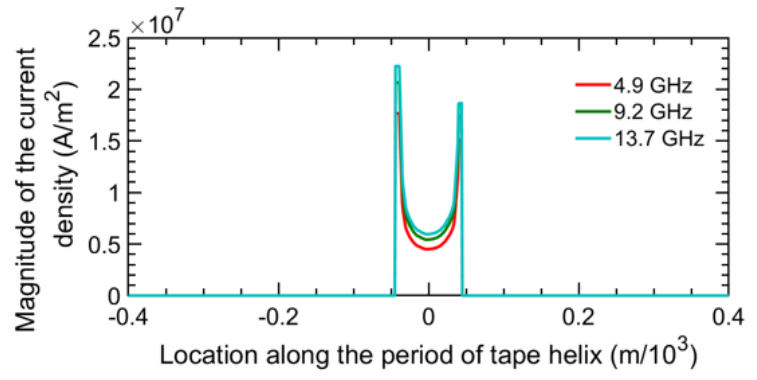

(a)

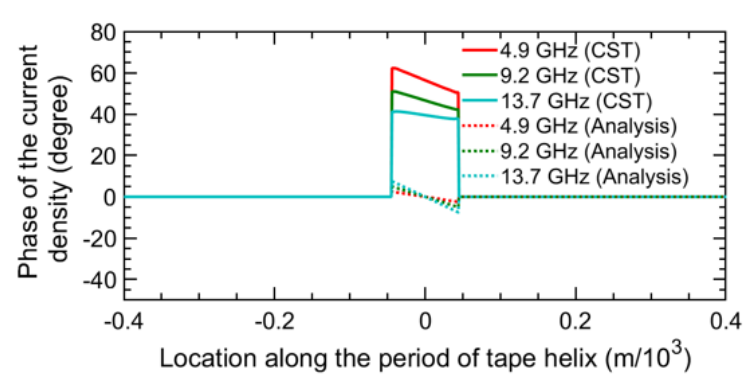

(b)

Figure 3.26: (a) Magnitude and (b) phase of the total current density along one period of tape helix for three different frequencies from simulation. This figure is for line-1.

The tape extends from $\mathrm{z}=-0.04 \mathrm{~mm}$ to $\mathrm{z}=+0.04 \mathrm{~mm}$. The values of phase from analysis for these three frequencies are also shown in (b). 
We take a look at the magnitude and phase of the total current density at two different locations on the tape which have different values of $\mathrm{z}$ within a single period of the helix tape. Although the current exists only on the tape, for the sake of completeness we show the actual magnitude and phase of the total current density along line-1 and line-2 (marked in Fig. 3.25) for the full period. With reference to Figs. $3.24 \& 3.25$, the axis of the helix coincides with the $z$-axis and the helix period extends from $z=-0.4 \mathrm{~mm}$ to $\mathrm{z}=+0.4 \mathrm{~mm}$. Line$1\left(\mathrm{r}=\mathrm{a}, \phi=90^{\circ}\right)$ and line- $2\left(\mathrm{r}=\mathrm{a}, \phi=180^{\circ}\right)$ extend over the entire period. Along line- 1 , the tape starts at $\mathrm{z}=-0.04 \mathrm{~mm}$ and ends at $\mathrm{z}=+0.04 \mathrm{~mm}$. Along line-2, the tape starts at $\mathrm{z}=+0.16$ $\mathrm{mm}$ and ends at $\mathrm{z}=+0.24 \mathrm{~mm}$.

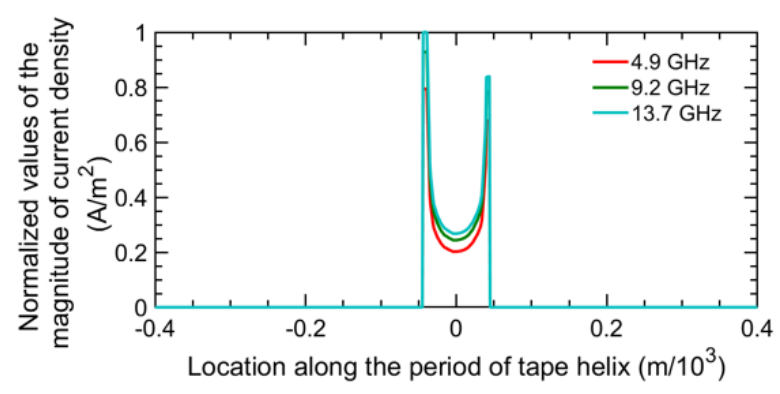

(a)

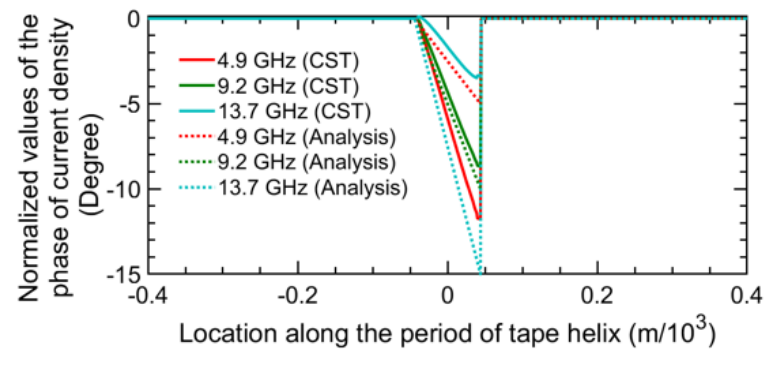

(b)

Figure 3.27: Normalized values of (a) magnitude and (b) phase of the total current density along one period of tape helix for three different frequencies from simulation.

This figure is for line- 1 . The tape extends from $z=-0.04 \mathrm{~mm}$ to $z=+0.04 \mathrm{~mm}$. The normalized values of the expected values of phase from analysis for these three frequencies are also shown in (b).

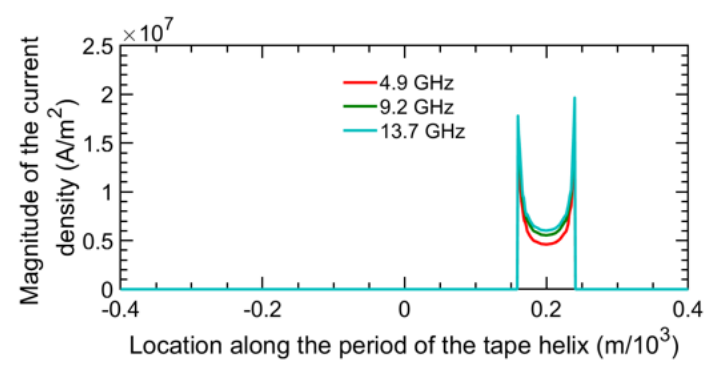

(a)

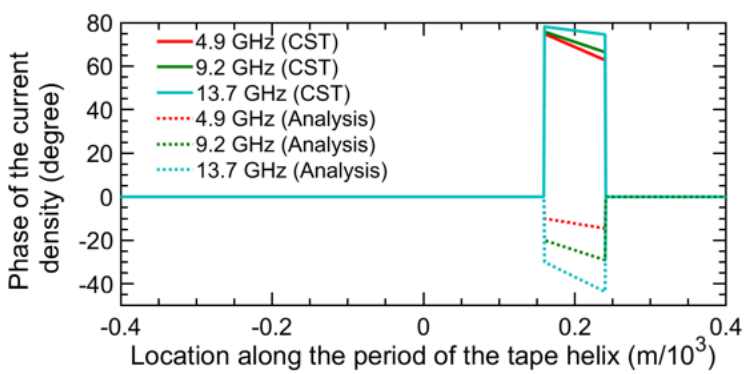

(b)

Figure 3.28: (a) Magnitude and (b) phase of the total current density along one period of tape helix for three different frequencies from simulation. This figure is for line-2. The tape extends from $\mathrm{z}=+0.16 \mathrm{~mm}$ to $\mathrm{z}=+0.24 \mathrm{~mm}$. The expected values of phase from analysis for these three frequencies are also shown in (b). 
The magnitude and phase of the total current density for three different frequencies (4.9 GHz, 9.2 GHz, and 13.7 GHz) along line-1 and line-2 are shown in Figs. 3.26-3.29. Actual magnitude and phase are shown in Figs. $3.26 \& 3.28$ and Figs. $3.27 \& 3.29$ show the normalized values. While the variation of phase is inherent in a propagating wave, here the magnitude of the total current density also varies with $\mathrm{z}$ to a small extent since the propagating wave consists of multiple space harmonics which have different phase constants. These figures imply that the nature of variation of the magnitude and phase is very similar for all locations on the tape.

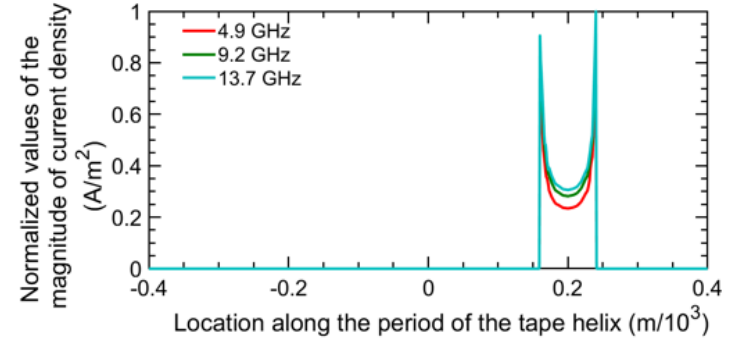

(a)

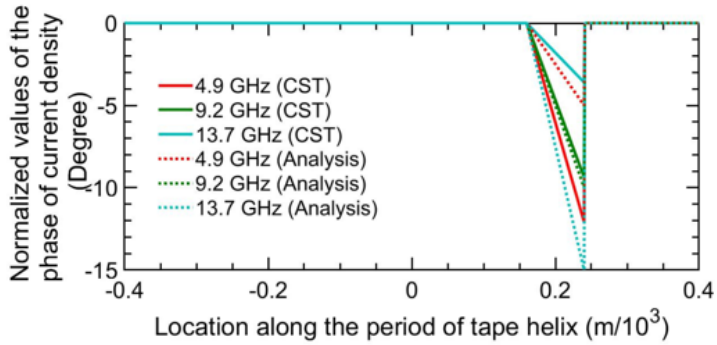

(b)

Figure 3.29: (a) Normalized values of (a) magnitude and (b) phase of the total current density along one period of tape helix for three different frequencies from simulation.

This figure is for line-2. The tape extends from $\mathrm{z}=+0.16 \mathrm{~mm}$ to $\mathrm{z}=+0.24 \mathrm{~mm}$. The normalized values of the expected values of phase from analysis for these three frequencies are also shown in (b).

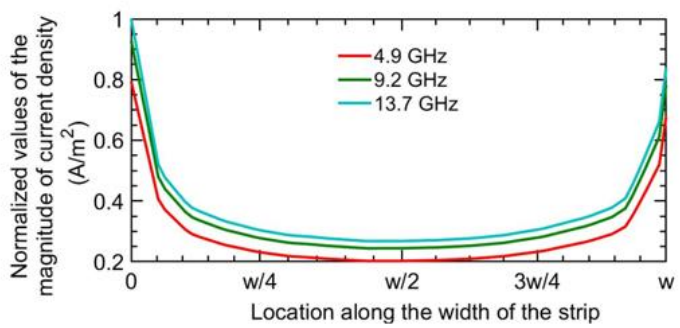

(a)

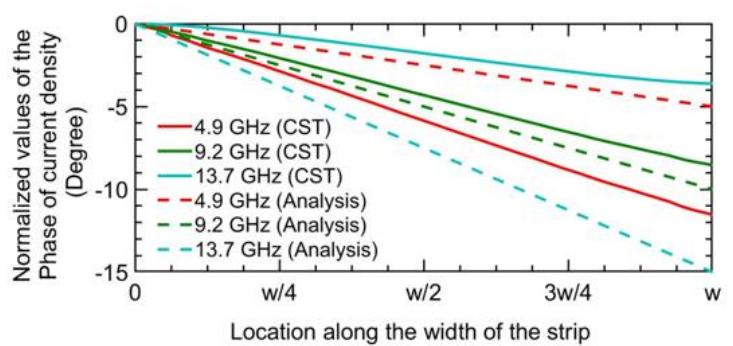

(b)

Figure 3.30: (a) Normalized values of (a) magnitude, and (b) phase of the total current density along the width of the tape for three different frequencies. This figure is for line-1. The tape extends from $\mathrm{z}=-0.04 \mathrm{~mm}$ to $\mathrm{z}=+0.04 \mathrm{~mm}$. So, $\mathrm{w}=0$ corresponds to $\mathrm{z}=-0.04 \mathrm{~mm}$ and $\mathrm{w}$ corresponds to $\mathrm{z}=+0.04 \mathrm{~mm}$. 
The version of Fig. 3.26 showing the normalized magnitude and phase of the total current density over the tape width alone is shown in Fig. 3.30. The magnitude curves are normalized with respect to the maximum value of current density for $13.7 \mathrm{GHz}$. Such a figure provides a clearer comparison between the nature of variation of the magnitude and phase from simulation and that expected from analysis.

As shown in Fig. 3.30(a), the concentration of current near the tape edges is much higher than that at the centre of the tape. This is a well-known phenomenon; therefore, the first assumption is incorrect. Similarly, as shown in Fig. 3.30(b), the variation of the phase along the tape width from simulation does not match with that from analysis. The phase of the current in the tape-helix should have the contribution from all space harmonics, since all space harmonics simultaneously exist in the EM wave propagating through the SWS.

It is clear that the nature of variation of magnitude and phase of the total current density along the tape width expected from analysis does not match those from simulations due to the approximations in the analysis. The assumption in the tape-helix analysis considers only the contribution of fundamental space harmonic, thus causing error in predicting the field values for the non-fundamental space harmonics.

Since the simulations do not use any of the simplifying assumptions, it seems reasonable to rely upon the results from simulation for both fundamental and nonfundamental space harmonics. The simulation of the helix with wider tape width and increased tape thickness also gives similar results. Thus, the conclusion that simulations provide more accurate estimates holds good for helices with wider and thicker tape as well.

\subsection{Interaction Impedance of Space Harmonics using Fourier Decomposition}

This section explains the procedure to evaluate the interaction impedance of the desired space harmonic in any SWS using simulation. Besides, the simulation results for the interaction impedance of the idealized circular helix of the previous section for the fundamental and the $n=-1$ space harmonic at axial and different off-axis positions are also presented in this section. Finally, the interaction impedance of a practical circular helix SWS for the fundamental and the $n=-1$ space harmonic at different positions is presented in this section. 


\subsubsection{Method to Find Interaction Impedance}

The first step to determine the interaction impedance of the desired space harmonic in a SWS using simulation is to evaluate the phase constant $\left(\beta_{0}\right)$ of the fundamental space harmonic and the total longitudinal electric field at the desired location in the SWS. In the second step, the phase constant $\left(\beta_{n}\right)$ of the desired space harmonic is evaluated using (3.2). In the third step, the magnitude of longitudinal electric field at a particular $(r, \phi)$ for the desired space harmonic $\left(\left|E_{z, n}(r, \phi)\right|\right)$ is evaluated using (3.21). Section 3.3.1 explains in a step-by-step fashion how to get $\left|E_{z, n}(r, \phi)\right|$ and $\beta_{n}$ for different space harmonics as a function of frequency. The next few steps are as follows.

The interaction impedance in a SWS for the desired space harmonic is calculated using (3.1):

$$
K_{c, n}=\frac{\left|E_{z, \mathrm{n}}(r, \phi)\right|^{2}}{2 P \beta_{n}^{2}}
$$

Once $\left|E_{z, n}(r, \phi)\right|$ and $\beta_{n}$ have been obtained, the only unknown quantity in (3.1) is the time-averaged RF power $P$ in the SWS, which can be easily obtained as [ 42], [97]:

$$
P=w V_{g}
$$

where, $w$ is the time averaged stored electromagnetic energy per unit length and $V_{g}$ is the group velocity. $V_{g}$ can be directly obtained by taking the slope of the tangent of dispersion diagram at the required frequency (Fig. 3.19); $w$ can be obtained as:

$$
w=\frac{W_{T}}{N L}
$$

where $W_{T}$ is the total energy, $N$ is the number of periods, and $L$ is the pitch of the SWS. CST Eigenmode simulations use single period of the SWS, hence $N$ is unity. The field value obtained from Eigenmode solver is normalized with $1 \mathrm{~J}$. Since the structure is lossless, and enclosed by PEC boundary, the $1 \mathrm{~J}$ energy is oscillating between $\mathrm{E}$ and $\mathrm{H}$ fields. Therefore $W_{T}$ is also unity in the Eigenmode simulations.

We give the simulation data in an 'excel' file as the input to MATLAB and implement the above steps in MATLAB to calculate the interaction impedance. 


\subsubsection{Results and Discussion}

\subsubsection{Ideal Circular Helix}

The interaction impedance for the fundamental space harmonic (Ko) at axial and different off-axis positions for the idealized circular helix of the previous section is given in Fig. 3.31. The figure shows the well-known behaviour of interaction impedance of the fundamental space harmonic. Ko is higher at lower frequencies and decreases with frequency; it also increases slightly as one moves closer to the helix. The figure also includes the results for interaction impedance of the circular helix at $r=a / 2$ that one would obtain without using Fourier decomposition, i.e., by using the total magnitude of $E_{z}$. The values of interaction impedance in this case become higher since we use the overestimated magnitude of $E_{z}$.

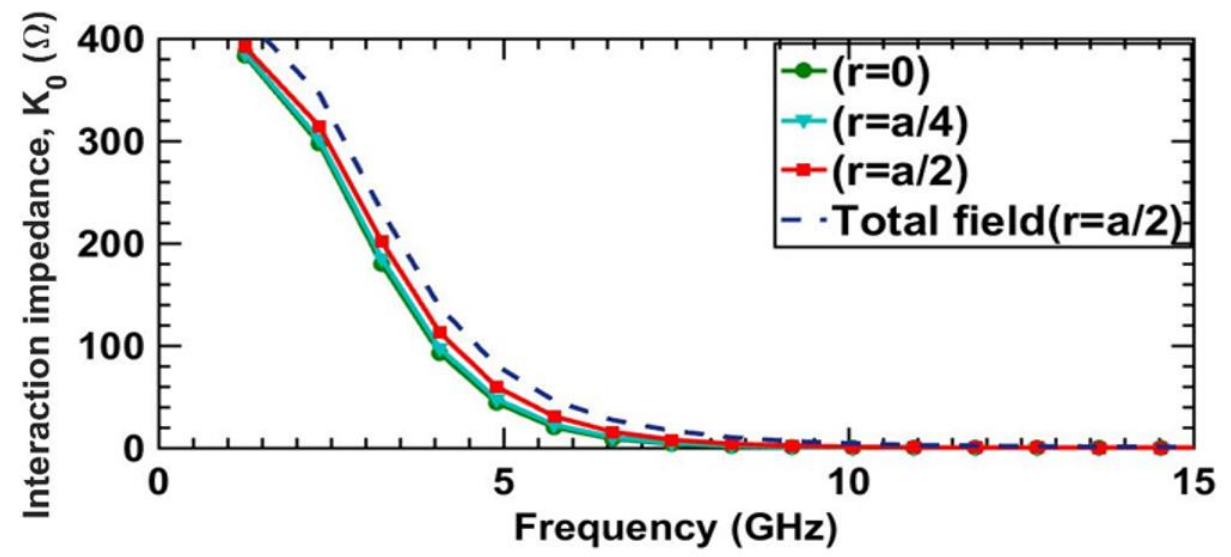

Figure 3.31: Simulation values of interaction impedance of the fundamental space harmonic in the circular helix at different positions.

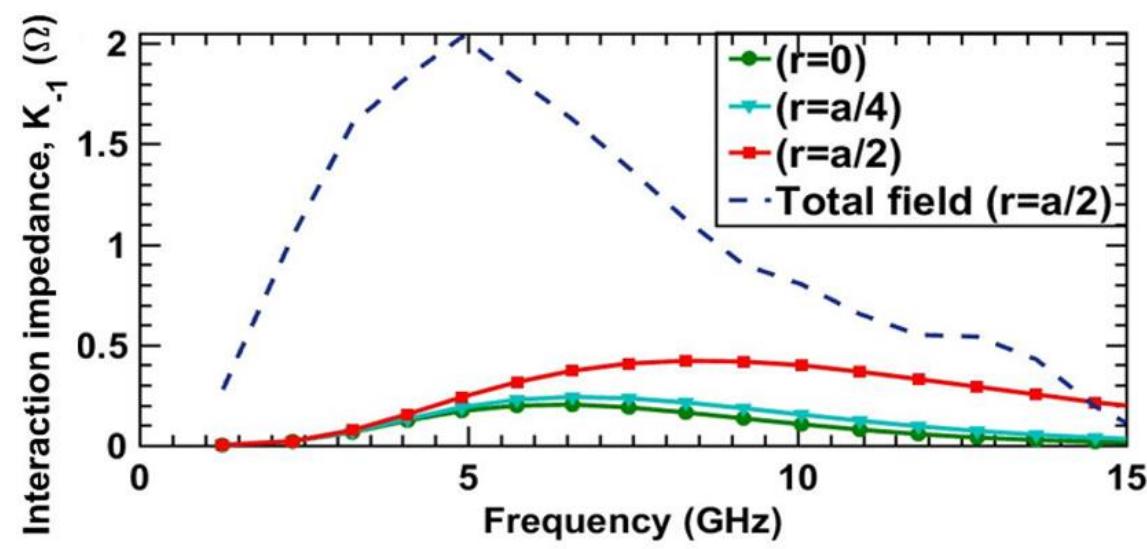

Figure 3.32: Simulation values of interaction impedance of the $n=-1$ space harmonic in the circular helix at different positions. 
Similarly, the interaction impedance for the $\mathrm{n}=-1$ space harmonic $\left(\mathrm{K}_{-1}\right)$ at axial and different off-axis positions for the idealized circular helix is given in Fig. 3.32. The variation of $\mathrm{K}_{-1}$ at relatively low frequencies is dominated by the variation of the phase constant for this space harmonic. The values of $\mathrm{K}_{-1}$ decrease with frequency after peaking at a certain frequency; this is because the fields in the helix tend to concentrate closer to the helix at higher frequency. The value of $\mathrm{K}_{-1}$ at a fixed frequency shows higher value at a position closer to the helix. Fig. 3.32 also includes the value of $\mathrm{K}_{-1}$ at $r=a / 2$ by using the magnitude of total $E_{z}$ from simulation. As expected, the interaction impedance of the n=1 space harmonic is significantly higher when we use the magnitude total $E_{z}$.

\subsubsection{Practical Circular Helix SWS}

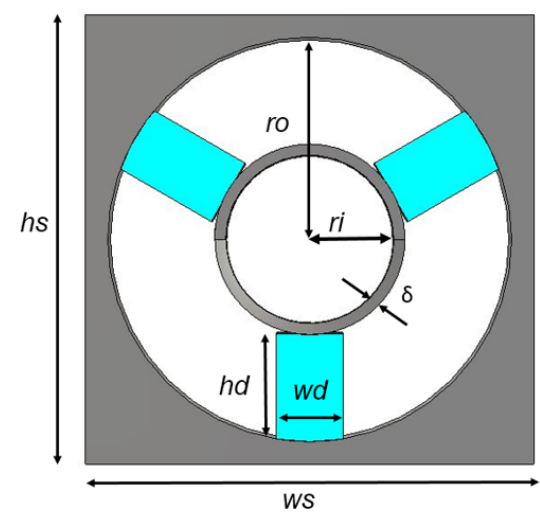

\begin{tabular}{|c|c|}
\hline Parameter & $\begin{array}{c}\text { Value } \\
(\mathrm{mm})\end{array}$ \\
\hline ri & 1.41 \\
\hline pitch & 0.8 \\
\hline $\mathrm{t}$ & 0.1 \\
\hline$\delta$ & 0.4 \\
\hline ro & 3.05 \\
\hline hd & 1.5 \\
\hline wd & 0.8 \\
\hline hs & 3.5 \\
\hline ws & 3.5 \\
\hline
\end{tabular}

Figure 3.33: Front view of the circular helix based practical slow-wave structure with dimensions.

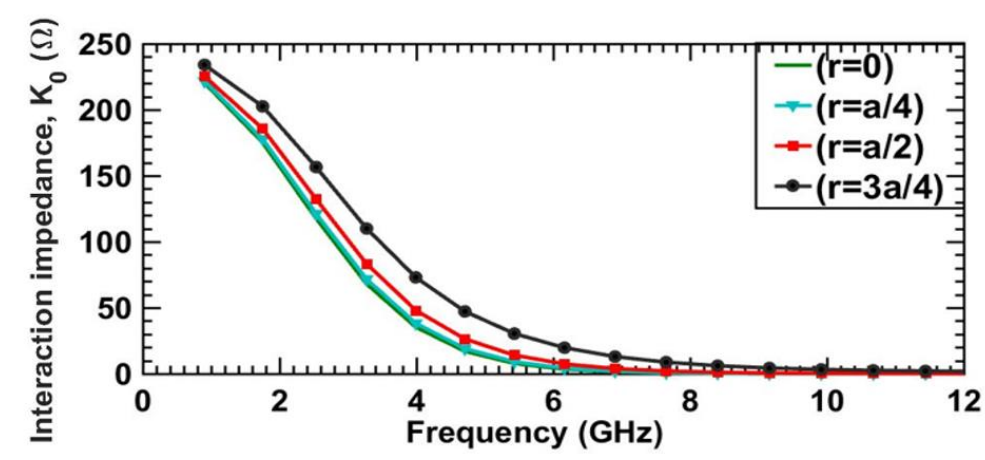

Figure 3.34: Simulation values of interaction impedance of the fundamental space harmonic in the practical structure at different positions.

The structure and dimensions of the practical circular helix SWS are shown in Fig. 3.33. The helix is supported inside a metal enclosure using three dielectric slabs of APBN material. The dielectric material has a dielectric constant of 5.1 and loss tangent of 0.005 . 
The CST eigenmode simulation can include the dielectric loss but the metal parts are considered lossless in the simulation since the CST eigenmode solver does not support lossy metals. The interaction impedance values for the fundamental and $n=-1$ space harmonic of this SWS are given in Fig. 3.34, and Fig. 3.35, respectively. The general trend of interaction impedance in this case also follows that in the ideal helix, i.e., Figs. 3.31 3.32. But the numerical values of interaction impedance and frequency range are different in this case due to the effect of dielectric slabs and metal enclosure.

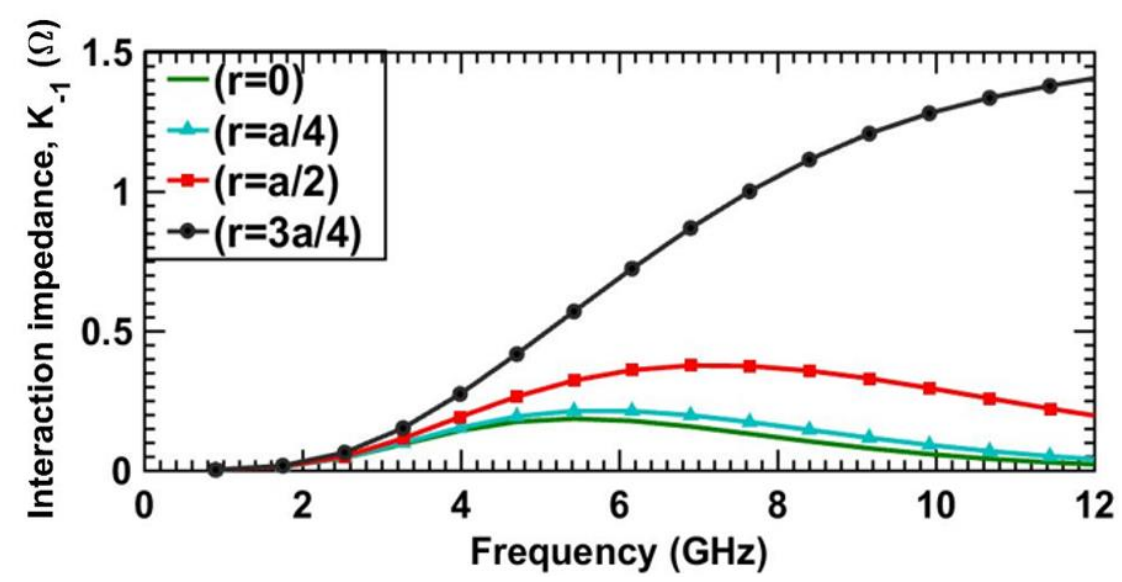

Figure 3.35: Simulation values of interaction impedance of the $n=-1$ space harmonic in the practical structure at different positions.

\subsection{Summary}

Fourier decomposition method is used with the simulation results to determine the interaction impedance for the fundamental and non-fundamental space harmonics of the circular helix SWS. The Fourier decomposition method was introduced for separating the magnitude of longitudinal electric field for the fundamental space harmonic from the total longitudinal electric field in a SWS by Kory and Dayton [95]. But that work was focused on the on-axis interaction impedance of the fundamental space harmonic. Here we have extended that work to determine the interaction impedance of the fundamental as well as the non-fundamental space harmonics at on-axis and off-axis positions of the SWS.

The comparison of the magnitude of longitudinal electric field shows that the normalized field values from simulation and the tape-helix analysis have a very good match for fundamental space harmonic but not for non-fundamental space harmonics. The current assumption in the tape-helix analysis only considers the effect of the fundamental space harmonic, which causes errors in the results of the non-fundamental space harmonics. Next, 
a method to extract the interaction impedance for different space harmonics in a SWS using simulation results and Fourier decomposition is explained. Illustrative simulation results for the interaction impedance of the circular helix for the fundamental and $n=-1$ space harmonic at axial and different off-axis positions are presented for an idealized circular helix with thin and narrow tape. Similar results are also presented for a practical circular helix SWS with a thicker and wider tape and supported with dielectric slabs inside a metal enclosure.

This method is used for evaluating the interaction impedance of various space harmonics in planar helix slow-wave structure with straight-edge connections (PH-SEC) in following chapters. Moreover, the method can be used for finding the interaction impedance of the desired space harmonic in any periodic SWS. 
Chapter 4 Tape-Helix Analysis of PH-SEC

\subsection{Introduction}

Even though circular helix is the most popular and widely used slow-wave structure (SWS) due to its strong beam-wave interaction over a wide bandwidth, its circular geometry is not easily amenable to printed circuit or microfabrication techniques. Besides, the fabrication of travelling-wave tubes (TWTs) at high frequencies becomes very difficult since the dimensions of the SWSs scale inversely with operating frequency. In this context, the planar helix SWS with straight-edge connections (PH-SEC) has been introduced [31]. As shown in Fig. 4.1, the PH-SEC is derived from rectangular helix by replacing its inclined edge connections with easily realizable straight-edge connections. The PH-SEC is easily amenable to printed circuit and microfabrication techniques due to its planar geometry.

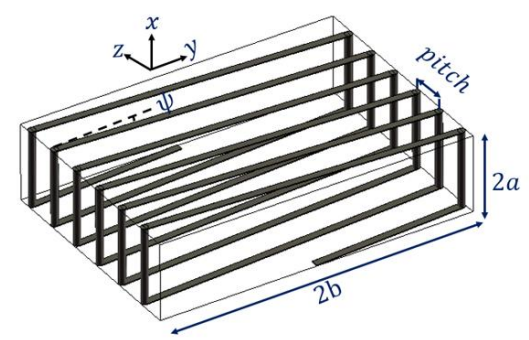

(a)

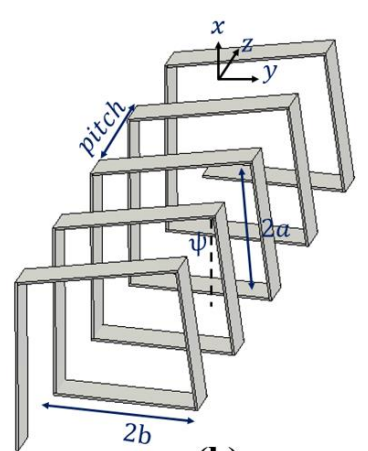

(b)

Figure 4.1: Perspective views of a) PH-SEC, and b) rectangular helix.

As mentioned in the previous chapters, the sheath-helix analysis and tape-helix analysis are the two established field theory-based analyses for studying the electromagnetic (EM) properties of circular helix. The sheath-helix analysis reduces the complexity in the analysis by neglecting the effect of tape width and the non-fundamental space harmonics in the SWS. The tape-helix analysis, although relatively more complex, improves the accuracy of results as compared to the sheath-helix analysis. The tape-helix model considers the helix as a perfectly conducting tape with finite width and infinitesimal thickness. The periodicity of the SWS is incorporated in the tape-helix analysis by using Fouquet's theorem.

The dispersion characteristics of PH-SEC have been studied using sheath-helix approximation and effective dielectric constant method (EDC) in [31], [98]. The EDC 
method was originally developed for reducing the complexity in the analysis of dielectric waveguides with rectangular cross-section. This method replaces the original 3-D waveguide by two related 2-D structures which are easy to analyse [99]. The results from the EDC method are accurate at a frequency range far from the cut-off.

A structure consisting of infinitely wide top and bottom strips of the PH-SEC has been analysed using the sheath-helix approximation in [100]. Later, the EDC method was used with the results in [100] to incorporate the effect of straight-edge connections in [31]. The EDC method has also been used for the analysis of PH-SEC with dielectric support and metal enclosure [98]. But the analysis of PH-SEC based on the tape-helix approximation, which would increase the accuracy of results by considering the effect of periodicity and tape width, has not been reported in the literature.

An initial approach for the tape-helix analysis of the infinitely wide planar helix $(\mathrm{PH})$ was reported in [101]. The PH is a 2-D structure and a simplified version of PH-SEC; it has only the top and bottom strips of the PH-SEC. The analysis in [101] leads to a complex derivation with double infinite series for field expressions. Further the tape-helix analysis of the rectangular helix has been reported recently [86]. This approach also leads to a complex analysis and rather complicated characteristic equation.

Here, we present a simplified analysis of PH-SEC using the tape-helix approximation. Compared to the analysis of planar helix in [101], the complexity in the derivation is reduced Moreover, the present analysis is simplified by applying the EDC method compared to the complicated analysis of rectangular helix in [86].

Discussion in Chapter 3 showed that the tape-helix analysis of the circular helix accurately predicts the dispersion characteristics and the interaction impedance of the fundamental space harmonic. Here we apply the tape-helix analysis to get more accurate results on the dispersion characteristics of the PH-SEC. The results of this analysis can be used for the initial selection of the dimensions of PH-SEC for a TWT with given target specifications.

Section 4.2 of this chapter presents the analysis of PH-SEC placed in a homogeneous medium using the tape-helix approximation. The application of EDC method to obtain the dispersion characteristics of PH-SEC are included in this section. The results obtained from the tape-helix analysis of PH-SEC are compared with those from CST simulation in Section 4.3. Comparison of both normalized phase velocity and interaction impedance of the 
fundamental space harmonic of PH-SEC is presented. In addition, design, fabrication, and measurement of a PH-SEC SWS in a homogeneous medium are also presented in this section. The measured results are compared with those obtained from the simulation and analysis. Section 4.3 also presents a comparison between the dispersion characteristics of the rectangular tape-helix obtained from the tape-helix analysis reported in [86] and those obtained from the present simplified analysis. This section ends with a comparison between the results obtained from the present analysis with those obtained from the analysis of the infinitely wide planar helix in [101].

The tape-helix analysis of PH-SEC in a metal enclosure is presented in Section 4.4. This section gives the derivation of characteristic equation, and the comparison of the results from analysis with the simulation. The chapter ends with a summary in Section 4.5.

\subsection{Tape-Helix Analysis of PH-SEC in a Homogeneous Medium}

This analysis considers the PH-SEC immersed in a homogeneous medium (free space). As shown in Fig. 4.1 (a), the PH-SEC has transverse dimensions of $2 a$ along the $x$ direction and $2 b$ along the $y$-direction. The period of a unit cell of PH-SEC along the $z$ direction is the pitch $L$. The EM wave in the structure is assumed to be travelling in the $z$-direction. The 3-D PH-SEC is divided into two related 2-D structures for simplifying the analysis using the EDC method. The first 2-D structure considers the top and bottom inclined strips of PH-SEC while the second 2-D structure considers the transverse confinement of PH-SEC due to the straight-edge connections. The following sub-sections present the analysis of infinitely wide planar helix using tape-helix approximation, application of the EDC method, and the estimation of interaction impedance of the fundamental space harmonic of PH-SEC.

\subsubsection{Tape-Helix Analysis of Infinitely Wide Planar Helix}

As shown in Fig. 4.2, the infinitely wide planar helix can be modelled as a pair of arrays of parallel, straight, and perfectly conducting strips. The strips are assumed to be infinitely long in both $y$ - and $z$-directions. As shown in Fig. 4.2 (a), the strips in the top array are oriented at an angle $+\psi$ with respect to the $y$-axis, while the strips in the bottom array are oriented at an angle $-\psi$ with respect to the $y$-axis. The top and bottom arrays are separated by a distance $2 a$ in the $x$-direction, which is indicated in Fig. 4.2 (b). The region outside arrays and the region between the arrays are considered to have the same material 
properties. The strips are assumed to have infinitesimally small thickness. These have a width of $w_{y}$ along the $y$-direction and $w z$ along the $z$-direction; $p_{y}$ and $p_{z}$ are the periodic spacing between the strips along the $y$ - and $z$-directions, respectively. The strip widths $w_{y}$ and $w_{z}$ are related to the actual strip width $w$ as:

$$
\begin{aligned}
& w_{y}=w / \sin \psi \\
& w_{z}=w / \cos \psi .
\end{aligned}
$$
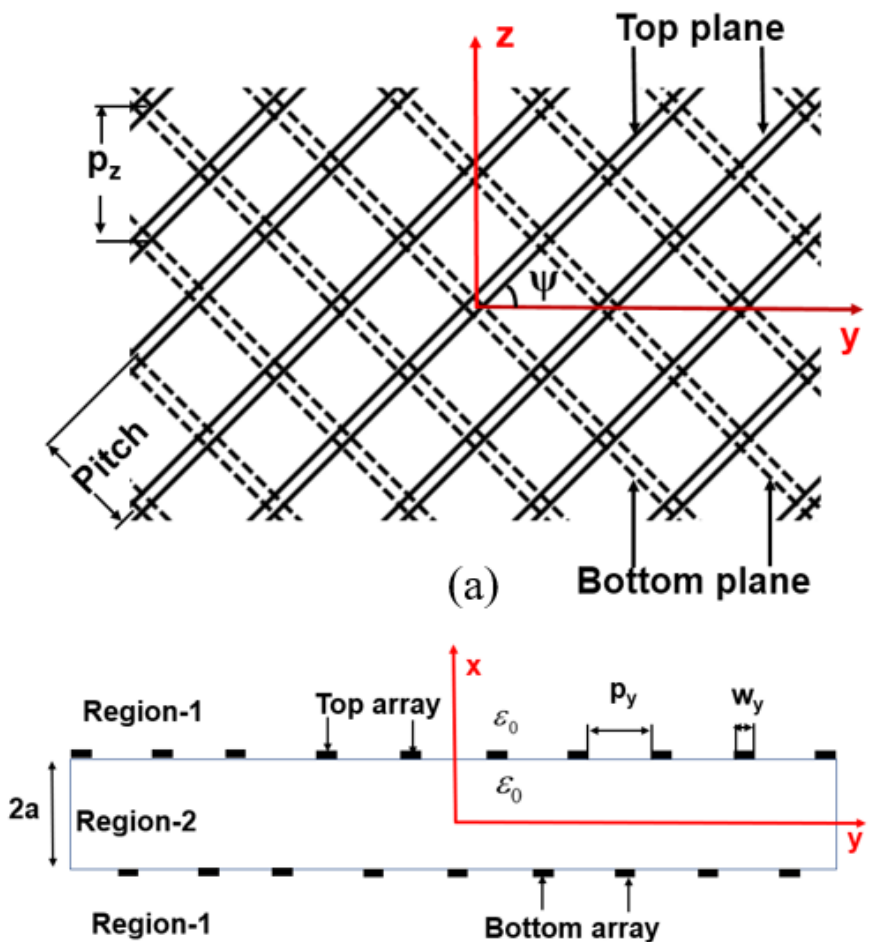

(b)

Figure 4.2: (a) Plan view and (b) cross-sectional view of infinitely wide planar helix.

The planar helix remains unchanged under any one or any combination of the following four transformations:

$$
\begin{aligned}
& (x, y, z) \rightarrow(-x,-y, z) \\
& (x, y, z) \rightarrow\left(x, y \pm \frac{p_{y}}{2}, z \pm \frac{p_{z}}{2}\right) \\
& (x, y, z) \rightarrow\left(x, y, z \pm p_{z}\right) \\
& (x, y, z) \rightarrow\left(x, y \pm p_{y}, z\right) .
\end{aligned}
$$

These four transformations constitute the symmetry properties of the $\mathrm{PH}$. By considering the four symmetric properties together, one can conclude that the structure 
supports transverse-symmetric and transverse-antisymmetric modes [102]. The transverse field components $\left(E_{x}, E_{y}, H_{x}, H_{y}\right)$ in the transverse-symmetric mode are symmetric with respect to $x$ while the longitudinal field components $\left(E_{z}, H_{z}\right)$ are anti-symmetric. In contrast, the transverse field components are anti-symmetric and longitudinal field components are symmetric with respect to $x$ in the transverse-antisymmetric mode. We are interested in the solution for the transverse-antisymmetric modes, since the operation of TWTs requires a non-zero value of the longitudinal electric field along the axis. Moreover, due to the inclined strips, the PH supports hybrid modes.

\subsubsection{FIELD EXPRESSIONS}

The Helmholtz's equation is used to obtain the field expressions for the transverseantisymmetric modes. Floquet's theorem is used for incorporating periodicity in the field expressions. The EM wave in the planar helix is assumed to have a time dependence of $e^{j \omega t}$ and is assumed to be propagating in the $z$-direction with the fundamental phase constant $\beta_{0}$. The general field expression for the longitudinal electric field in the planar helix can be written as:

$$
E_{z}=\sum_{n=-\infty}^{\infty} \sum_{m=-\infty}^{\infty} f(x) e^{\left\{j \frac{2 \pi n y}{p_{y}}\right\}} e^{-j\left\{\frac{2 \pi m z}{p_{z}}+\beta_{0} z\right\}}
$$

where, $f(x)$ is a function of $x$. The space harmonic number in the $y$-and $z$-direction are $n$, and $m$, respectively. Such a field expression, having a double infinite series, leads to a complex analysis. The complexity in the analysis is reduced by assuming a similar space harmonic distribution in both $y$-and $z$-directions, i.e. $n=m$.

The simplified field expressions for the longitudinal electric and magnetic fields for the transverse-antisymmetric (longitudinal symmetric) mode in the two regions are:

$$
\begin{aligned}
& E_{z 1}=\sum_{n=-\infty}^{\infty} A_{n} \exp \left\{-u_{n}(x-a)\right\} e^{\left\{j \frac{2 \pi n y}{p_{y}}\right\}} e^{\left\{-j \beta_{n} z\right\}} \\
& E_{z 2}=\sum_{n=-\infty}^{\infty} B_{n} \cosh \left(u_{n} x\right) e^{\left\{j \frac{2 \pi n y}{p_{y}}\right\}} e^{\left\{-j \beta_{n} z\right\}} \\
& H_{z 1}=\sum_{n=-\infty}^{\infty} C_{n} \exp \left\{-u_{n}(x-a)\right\} e^{\left\{j \frac{2 \pi n y}{p_{y}}\right\}^{\left\{-j \beta_{n} z\right\}}} \\
& H_{z 2}=\sum_{n=-\infty}^{\infty} D_{n} \cosh \left(u_{n} x\right) e^{\left\{j \frac{2 \pi n y}{p_{y}}\right\}^{\left\{-j \beta_{n} z\right\}}}
\end{aligned}
$$


where subscripts 1 and 2 represent the field expression for region-1 (outside the arrays), and region-2 (between the arrays), respectively; $n$ is the number of space harmonic. $A_{n}, B_{n}$, $C_{n}$, and $D_{n}$ are the unknown amplitude constants for the $n^{\text {th }}$ space harmonic. $\beta_{n}$ is the phase constant of the $n^{\text {th }}$ space harmonic, which is related to the phase constant of the fundamental space harmonic $\beta_{0}$ as $\beta_{n}=\beta_{0}+\left(2 \pi n / p_{z}\right) . u_{n}$ is the decay constant in the $x$-direction, obtained by the following equation:

$$
u_{n}^{2}=\left(\frac{2 \pi n}{p_{y}}\right)^{2}+\beta_{n}^{2}-k_{0}^{2}
$$

where $k_{0}=\omega\left(\mu_{0} \varepsilon_{0}\right)^{1 / 2}$ is the wave number in vacuum at the angular frequency $\omega$.

The field expressions for the transverse field components can be obtained from the field expressions of the longitudinal fields (4.8a) -(4.8d) and Maxwell's equations. The relations between the longitudinal fields and the transverse fields, as well as the field expressions for the transverse fields, are given in Appendix C. The unknown amplitude constants in the field expressions can be obtained using appropriate boundary conditions.

\subsubsection{BOUNDARY CONDITIONS}

As shown in Fig. 4.2, the planar helix is symmetric about $x=0$ plane. Hence we need to consider only the upper half of the structure $(x \geq 0)$ for applying the boundary conditions. Continuity of the tangential electric field and discontinuity of the tangential magnetic field at $x=a$ gives the following boundary conditions:

$$
\begin{aligned}
& E_{z 1, n}(x=a)=E_{z 2, n}(x=a) \\
& E_{y 1, n}(x=a)=E_{y 2, n}(x=a) \\
& {\left[H_{z 2, n}(x=a)-H_{z 1, n}(x=a)\right]=J_{y, n}} \\
& {\left[H_{y 1, n}(x=a)-H_{y 2, n}(x=a)\right]=J_{z, n}}
\end{aligned}
$$

where $J_{y, n}$ is the surface current density of the $n^{\text {th }}$ space harmonic at $x=a$ interface in the $y$-direction. Similarly, $J_{z, n}$ is the surface current density of the $n^{\text {th }}$ space harmonic at $x=a$ in the $z$-direction. Similar to the tape-helix analysis of the circular helix presented in Chapter 3, we assume that the current in the strips flows only along the direction of the strips. $J_{y, n}$ and $J_{z, n}$ can be expressed in terms of the surface current density $J_{\| n}$ of the $n^{\text {th }}$ space harmonic at $x=a$ interface in the direction of the strips as: 


$$
\begin{gathered}
J_{z, n}=J_{\| n} \sin \psi \\
J_{y, n}=J_{\| n} \cos \psi .
\end{gathered}
$$

The total current is assumed to have a phase variation only according to the fundamental space harmonic $\left(e^{-j \beta_{0} z}\right)$ and a constant magnitude $J$ along the strip width. The total surface current is $J e^{-j \beta_{0} z}$ along the strip width and zero in the remaining regions. $J_{\| n}$ can be obtained by taking the Fourier components of the total current on the strips as:

$$
J_{\| n}=\frac{J w_{z}}{p_{z}} s i \mathrm{nc}\left(\frac{\beta_{n} w_{z}}{2}\right) \text {. }
$$

The unknown amplitude constants are obtained by considering the nontrivial solution while applying the boundary conditions as:

$$
\begin{aligned}
A_{n} & =\frac{-k_{C}^{2} \cosh \left(u_{n} a\right)\left(j \omega \mu_{0}\right) u_{n} J_{\| n}}{u_{n}^{2} k^{2} e^{u_{n} a}}\left[\sin \psi+\frac{1}{k_{C}^{2}}\left(\frac{2 \pi n}{p_{y}}\right) \beta_{n} \cos \psi\right] \\
B_{n} & =\frac{A_{n}}{\cosh \left(u_{n} a\right)} \\
C_{n} & =\frac{-J_{\| n} \cos \psi \sinh \left(u_{n} a\right)}{e^{u_{n} a}} \\
D_{n} & =\frac{-C_{n}}{\sinh \left(u_{n} a\right)}
\end{aligned}
$$

where $k_{C}^{2}=k_{0}^{2}-\beta_{n}^{2}$.

\subsubsection{ChARACTERISTIC EQUATION}

The characteristic equation for determining $\beta_{0}$ for the infinitely wide planar helix is obtained using the condition $E_{1 \|}(x=a)=0$ at the centre of the strips. $E_{1 \|}$ is the electric field intensity parallel to the strips and is given as:

$$
E_{1, \|}=\sum_{n=-\infty}^{+\infty} E_{1, \| n}=\sum_{n=-\infty}^{+\infty}\left(E_{y 1, n} \cos \psi+E_{z 1, n} \sin \psi\right)
$$

The resulting characteristic equation for the infinitely wide planar helix is obtained as:

$$
\sum_{n=-\infty}^{+\infty}\left\{\begin{array}{c}
\frac{\left(j \omega \mu_{0}\right) u_{n} \cos ^{2} \psi}{k_{c}^{2} e^{u_{n} a}}\left(\frac{J w_{z}}{p_{z}} \operatorname{sinc}\left(\frac{\beta_{n} w_{z}}{2}\right)\right) \\
{\left[\sinh \left(u_{n} a\right)-\frac{\cosh \left(u_{n} a\right)}{u_{n}^{2} k_{o}{ }^{2}}\left(\frac{2 \pi n}{p_{y}} \beta_{n}+k_{c}^{2} \tan \psi\right)^{2}\right]}
\end{array}\right\}=0 .
$$

Even though (4.15) has infinite number of space harmonics, its numerical solution converges by considering the first seven space harmonics. 
4.2.2 Effective Dielectric Constant (EDC) Method

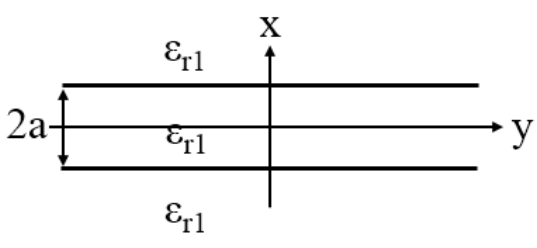

(a)

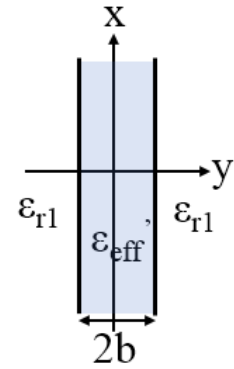

(b)

Figure 4.3: Model for applying EDC method: (a) $x$-dependent profile; (b) $y$-dependent profile.

The dispersion characteristics of the PH-SEC can be obtained using the EDC method. The PH-SEC shown in Fig. 4.1(a) can be analysed using two steps named as $x$-dependent profile and $y$-dependent profile. As shown in Fig. 4.3 (a), the $x$-dependent profile considers the infinitely wide planar helix which is confined only in the $x$-direction. The $y$-dependent profile, shown in Fig. 4.3 (b), considers an infinitely long dielectric slab which is confined only in the $y$-direction.

\subsubsection{1 $X$-DEPENDENT PROFILE}

The $x$-dependent profile uses the characteristic equation of the infinitely wide planar helix, which is described in the previous sub-section. An infinitely wide planar helix has a constant pitch angle for all parts of the structure. But this does not hold for PH-SEC due to the straight-edge connections. Therefore, the concept of an 'effective pitch angle' is used in the analysis to include the slowing down effect of the PH-SEC correctly. The effective pitch angle for the PH-SEC is calculated as:

$$
\psi_{\text {eff }}=\tan ^{-1}\left(\frac{\text { Period of } P H-S E C}{\text { Perimeter of } P H-S E C}\right)=\tan ^{-1}\left(\frac{L}{4(a+b)}\right) .
$$

The $x$-dependent profile uses the value of the effective pitch angle from (4.16) in the characteristic equation (4.15) of the infinitely wide planar helix. By numerically solving (4.15), the phase constant of the fundamental space harmonic in the infinitely wide planar helix, $\beta_{A}$, can be obtained. The effective dielectric constant $\varepsilon_{e f f}$ for the second step of the EDC method can be obtained from $\beta_{A}$ as:

$$
\varepsilon_{e f f}=\left(\frac{\beta_{A}}{k_{0}}\right)^{2} .
$$


The phase constant $\beta_{A}$ calculated from the $x$-dependent profile is accurate for a $\mathrm{PH}$ SEC except at relatively low frequencies where the effect of the transverse truncation is more critical. The $y$-dependent profile, described in the next sub-section, takes into account the effect of the transverse truncation, making the results more accurate at lower frequencies.

\subsubsection{Y-DEPENDENT PROFILE}

This step considers a dielectric slab which is $2 b$ wide along the $y$-direction and is infinitely long along the $x$-direction. The slab is considered to have a dielectric constant $\varepsilon_{e f f}$ and is immersed in free space. The dielectric constant $\varepsilon_{e f f}$ is calculated from the $x$-dependent profile using (4.17). The dielectric slab can support both TE and TM modes and they can be even or odd with respect to the $y$-axis. To support the longitudinal-symmetric mode of the original structure, we consider TE even mode of the dielectric waveguide [31]. This mode has even variation of $E_{x}$ with respect to $y$ and is the lowest order mode for a dielectric slab. The $y$-dependent profile gives phase constant $\beta_{B}$ by using the following relations [103]:

$$
\begin{aligned}
& \frac{v_{1}}{v_{2}}=\tan \left(v_{2} b\right) \\
& \beta_{B}^{2}=v_{1}{ }^{2}+k_{1}{ }^{2}=k_{\text {eff }}^{\prime 2}-v_{2}{ }^{2}
\end{aligned}
$$

where $k_{1}^{2}=\omega^{2} \mu_{0} \varepsilon_{0}$ and $k_{e f f}^{\prime}{ }^{2}=\omega^{2} \mu_{0} \varepsilon_{0} \varepsilon_{r, \text { eff }}^{\prime}$ are the wave numbers in free space and dielectric slab, respectively; $v_{1}$ and $v_{2}$ are the y-direction decay coefficients for the free space and dielectric regions, respectively.

Finally, the overall phase constant $\beta$ of PH-SEC is obtained by weighted averaging of $\beta_{A}$ and $\beta_{B}$ as:

$$
\beta=\frac{b \beta_{A}+a \beta_{B}}{a+b}
$$

\subsubsection{Interaction impedance}

The on-axis interaction impedance for the fundamental space harmonic in a SWS is given as:

$$
K_{0}=\frac{\left|E_{z, 0}^{2}(0)\right|}{2 P \beta_{0}^{2}}
$$


where $\left|E_{z, 0}(0)\right|$ is the magnitude of longitudinal component of the electric field at the centre of the SWS for the fundamental space harmonic and $P$ is the total RF power propagating through the SWS. The total RF power is the sum of the power contributed by all space harmonics. We consider the sum of power contributed by the first seven space harmonics as the total power since the power contributed by higher order harmonics is negligibly small. The power flow per unit width corresponding to the $n^{\text {th }}$ space harmonic for an infinitely wide planar helix shown in Fig. 4.2 is given as:

$$
P_{u w, n}=\frac{1}{2} \operatorname{Re}\left[\int_{x}\left(E_{x, n} \cdot H_{y, n}^{*}-E_{y, n} \cdot H_{x, n}^{*}\right) d x\right] .
$$

The total power flow in PH-SEC is obtained by multiplying $P_{u w, n}$ by $2 b$, i.e., the width in the $y$-direction:

$$
P_{n}=P_{u w, n} \times 2 b
$$

The interaction impedance for PH-SEC can be directly obtained by substituting the results of phase constant of PH-SEC from (4.20) and field expressions of infinitely wide planar helix in (4.8) and Appendix C in (4.21) - (4.23).

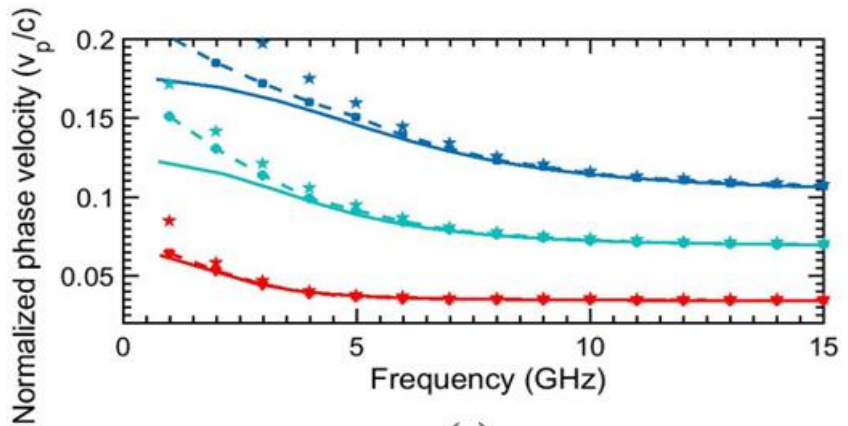

(a)

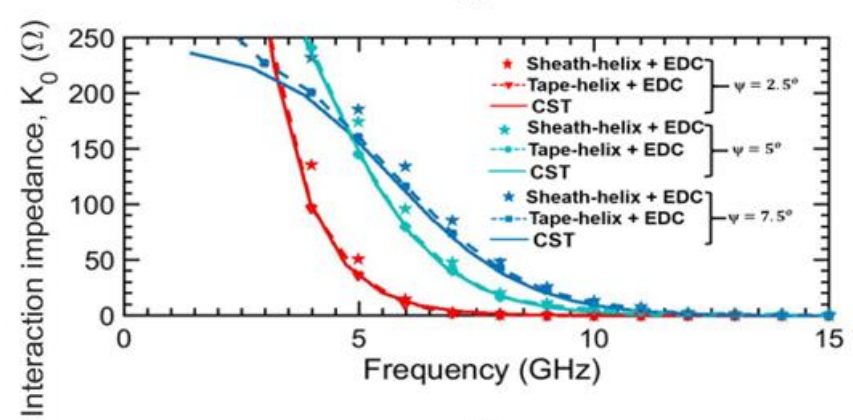

(b)

Figure 4.4: Comparison of simulated and analytical results for a PH-SEC for three different pitch angles $\left(\psi=2.5^{\circ}, 5^{\circ}, 7.5^{\circ}\right)$ with $a / b=0.25, a=0.48 \mathrm{~mm}$, and $w / L=0.2$.

(a) Normalized phase velocity, (b) Interaction impedance. 


\subsection{Results and discussion}

The results from the above analysis are compared with those from simulation, measurement, tape-helix analysis of rectangular helix [86], and the tape-helix analysis of infinitely wide planar helix [101]. The results of these comparisons are presented in the following sub-sections.

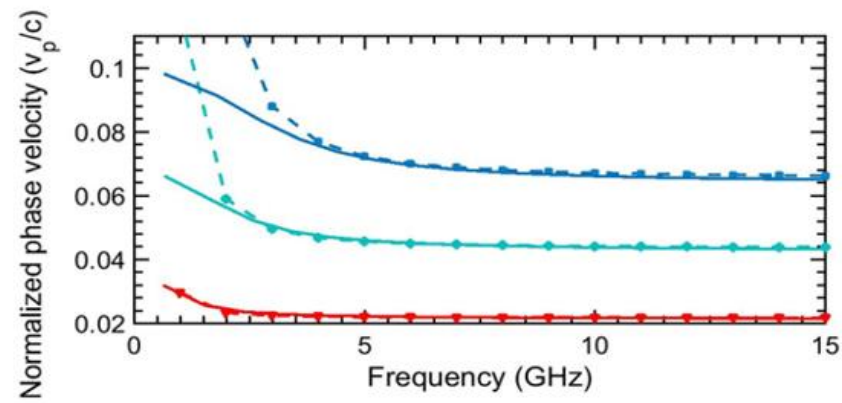

(a)

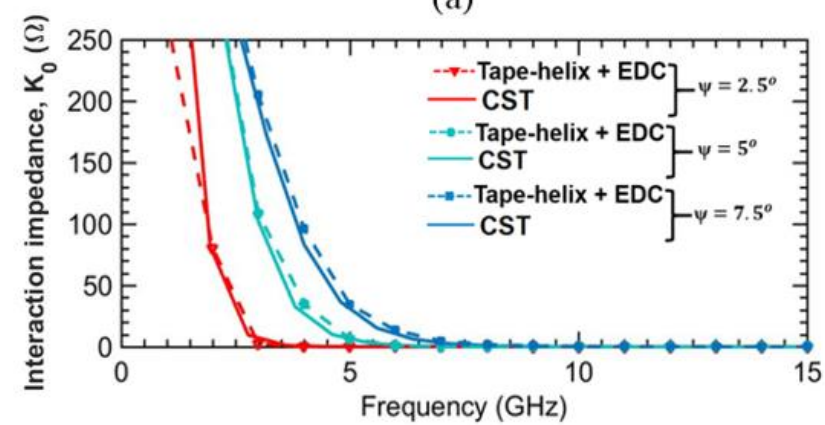

(b)

Figure 4.5: Comparison of simulated and analytical results for a PH-SEC for three different pitch angles $\left(\psi=2.5^{\circ}, 5^{\circ}, 7.5^{\circ}\right)$ with $a / b=1, a=1.2 \mathrm{~mm}$, and $w / L=0.2$. (a)

Normalized phase velocity, (b) Interaction impedance.

Fig. 4.4 show the results for PH-SEC with $a / b=0.25$ and $a=0.48 \mathrm{~mm}$. The normalized phase velocity from analysis shows a good match with simulation results for all three pitch angles. The result shows some inaccuracy at relatively low frequencies; this is due to the well-known inaccuracy of EDC method at frequencies below cut-off. As shown in Fig. 4.4 (b), the interaction impedance from the analysis also shows a good match with simulation results.

Fig. 4.4 also includes the normalized phase velocity and interaction impedance of the PH-SEC obtained from the sheath-helix approximation with EDC [31]. As shown in the figure, the results from the tape-helix approximation with EDC are more accurate than the results from the sheath-helix approximation with EDC. From the results shown in Fig. 4.4 
we observe that the phase velocity values from tape-helix analysis provides maximum improvement at lower frequency region. For the phase velocity of the PH-SEC with $\psi=7.5^{\circ}$, the tape-helix model gives a maximum improvement of $20 \%$ at the lower frequency end and minimum improvement of $4 \%$ at the higher frequency end over the sheath-helix analysis. The interaction impedance also has similar percentage improvements.

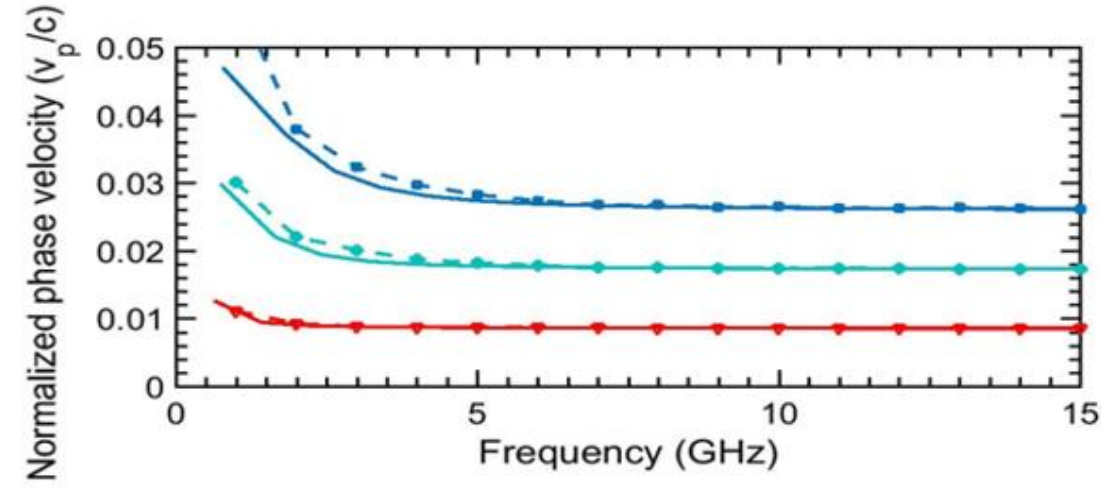

(a)

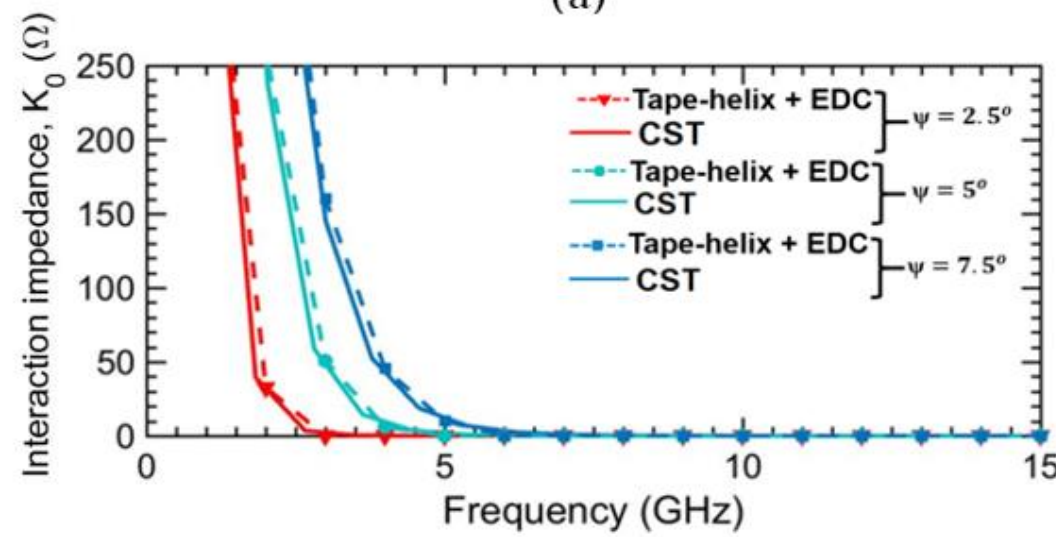

(b)

Figure 4.6: Comparison of simulated and analytical results for a PH-SEC for three different pitch angles $\left(\psi=2.5^{\circ}, 5^{\circ}, 7.5^{\circ}\right)$ with $a / b=4, a=1.92 \mathrm{~mm}$, and $w / L=0.2$. (a)

Normalized phase velocity, (b) Interaction impedance.

Fig. 4.5 and Fig. 4.6 show the results for PH-SECs with $a / b=1$ and $a=1.2 \mathrm{~mm}$, and $a / b=4$ and $a=1.92 \mathrm{~mm}$, respectively. Similar to the results in Fig. 4.4, the normalized phase velocity from analysis in these cases also shows a good match with simulation results except at relatively low frequencies. The interaction impedance of the fundamental space harmonic also shows a close match with the results from simulation. As shown in Fig. 4.4 - Fig. 4.6, irrespective of the aspect ratio, the phase velocity of PH-SEC increases with pitch angle. The plots also show that the PH-SECs with higher pitch angle can provide higher interaction impedance. 


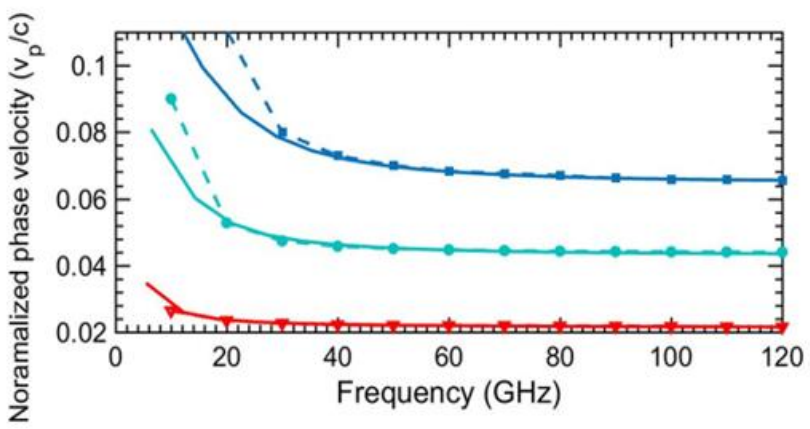

(a)

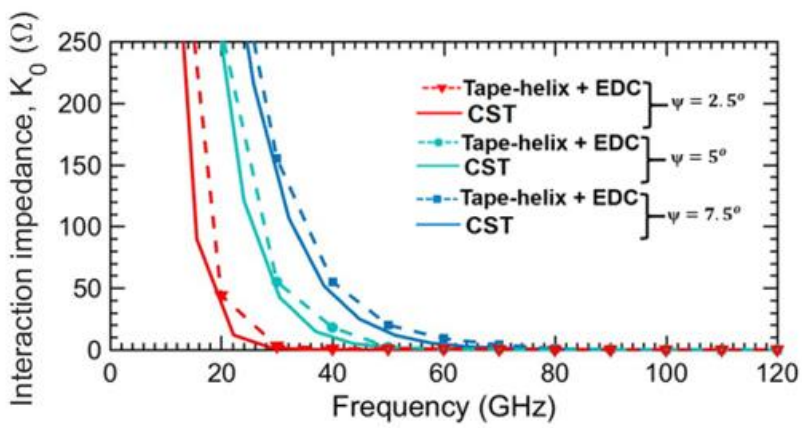

(b)

Figure 4.7: Comparison of simulated and analytical results at W-band for a PH-SEC for three different pitch angles $\left(\psi=2.5^{\circ}, 5^{\circ}, 7.5^{\circ}\right)$ with $a / b=1, a=0.15 \mathrm{~mm}$, and $w / L=0.2$.

(a) Normalized phase velocity, (b) Interaction impedance.

The results from tape-helix analysis are also compared with simulation results for a PH-SEC operating at W-band. As shown in Fig. 4.7, the normalized phase velocity and interaction impedance of a PH-SEC operating at W-band are compared for three different pitch angles $\left(\psi=2.5^{\circ}, 5^{\circ}, 7.5^{\circ}\right)$. The PH-SEC has $a / b=1, a=0.15 \mathrm{~mm}$, and the strip width as $20 \%$ of the pitch. Similar to the results in Fig. 4.4 - Fig. 4.6, here also the normalized phase velocity from analysis closely matches with the results from simulation except at relatively low frequencies. Moreover, the interaction impedance from the tape-helix analysis shows a good match with the results from simulation. These results indicate that the tape-helix analysis of PH-SEC can provide accurate results for frequencies in the range of 100 s of GHz.

The dispersion diagram for a PH-SEC with $a / b=1, a=L=1.2 \mathrm{~mm}$, and strip width $=0.24 \mathrm{~mm}$ is given in Fig. 4.8. Similar to the dispersion diagram of other periodic SWSs, this diagram also shows the existence of passbands and stop bands. These results again confirm that the tape-helix analysis can provide accurate results for the PH-SEC for a wide range of pitch angles and aspect ratios. 


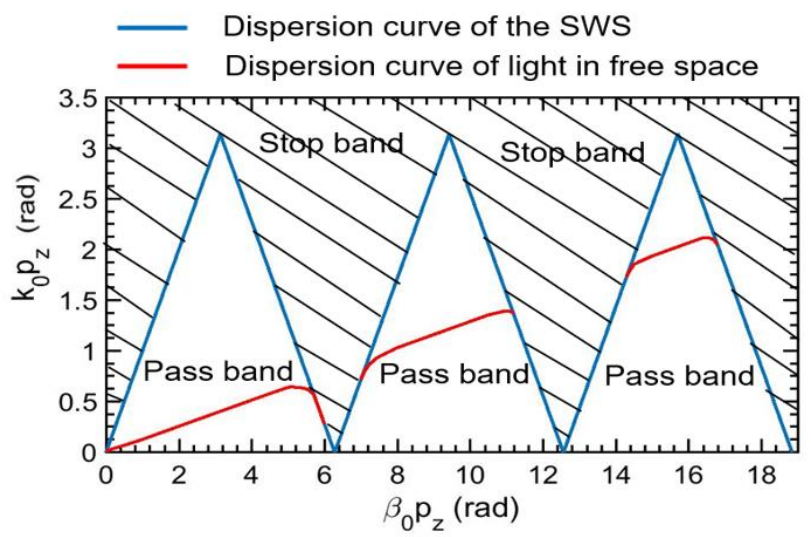

Figure 4.8: Dispersion diagram of a PH-SEC with $a / b=1, a=1.2 \mathrm{~mm}, w / L=0.2$, and $a / L=1$.

\subsubsection{Experimental Validation of the Analysis}

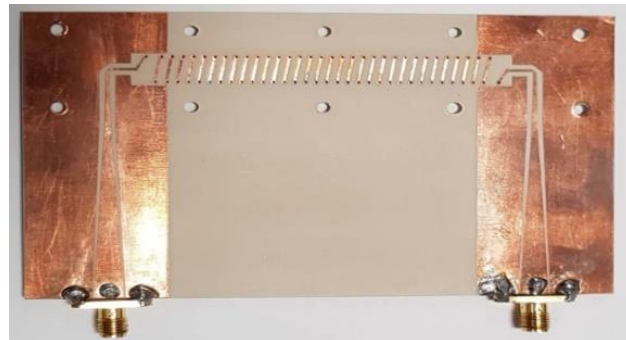

(a)

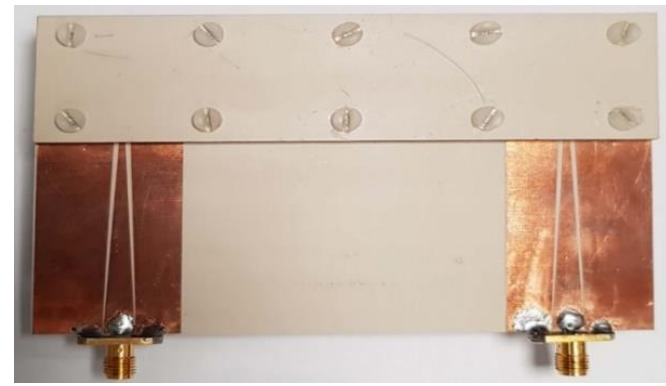

(b)

Figure 4.9: Fabricated prototype of the SWS with 30 periods. (a) The SWS before assembling top and bottom dielectric sheets; (b) the final SWS.

The above analysis presents the dispersion characteristics of PH-SEC immersed in a homogeneous medium. Therefore, we designed and fabricated a PH-SEC that substantially emulates a PH-SEC immersed in a homogeneous dielectric medium for an experimental validation of the analysis. RO3203 substrate with thickness of $1.524 \mathrm{~mm}$, dielectric constant of 3.03, and loss tangent of 0.0016 is used for the fabrication. A PH-SEC with a= $0.762 \mathrm{~mm}, b=3 \mathrm{~mm}$, pitch $=2.1 \mathrm{~mm}$, and strip width of $0.6 \mathrm{~mm}$ is designed and fabricated. 
Plated through holes (PTH) with radius of $0.25 \mathrm{~mm}$ are used for realizing the straight-edge connections of the PH-SEC. The straight-edge connections and the horizontal strips of the $\mathrm{PH}-\mathrm{SEC}$ are connected together using circular patches of radius $0.4 \mathrm{~mm}$.

Two SWSs with different lengths but otherwise identical have been designed and fabricated. The first SWS has 25 periods of PH-SEC while the second one has 30 periods. Fig. 4.9 (a) shows the fabricated prototype of the SWS with 30 periods of PH-SEC. As shown in the figure, coplanar waveguides are used to connect the SWS with the SMA connectors. The width of the coplanar waveguide is tapered to attain a wideband matching. Besides, different pitch lengths are used for two periods at both ends of the SWS to attain better matching. As shown in Figs. 4.9 (b), two layers of RO3203 substrate are attached above and below the SWS using nylon screws for approximating the SWS immersed in a homogeneous dielectric medium.

The S-parameters of the SWS are obtained from the Transient solver in the CST Microwave Studio. The Transient solver is a time domain solver. As shown in Fig. 4.9, the 3D geometry of the SWS is simulated in this solver. Open boundary condition (available in this solver) is applied all around the SWS since the structure is in free space. The width of the CPW feed line is gradually tapered to match 50 -ohm measurement system. Waveguide ports in the simulator are used to excite the CPW feed lines. In cases where the SWS is enclosed in a metal shield, one applies the PEC boundaries.

We can input the frequency range over which one needs to simulate the SWS. The Transient solver applies a suitable time-domain signal (which covers the required frequency range) as the input signal at the input port of the device and solves the Maxwell's equations to simulate the corresponding time-domain output signals at the input as well as the output ports. The solver calculates the frequency-domain S-parameters of the structure from the time-domain signals. Hexahedral mesh in the solver is used with mesh refinement for converged results. Losses in the SWS are considered by specifying the conductivity of metal and loss tangent of dielectric material.

\subsubsection{CONVERGENCE OF TRANSIENT SOLVER}

The convergence with respect to the number of mesh cells for obtaining the transmission parameters of the SWS from Transient solver is shown below. We have used hexahedral mesh cells ranging from 841780 to 2067520 for convergence study. 


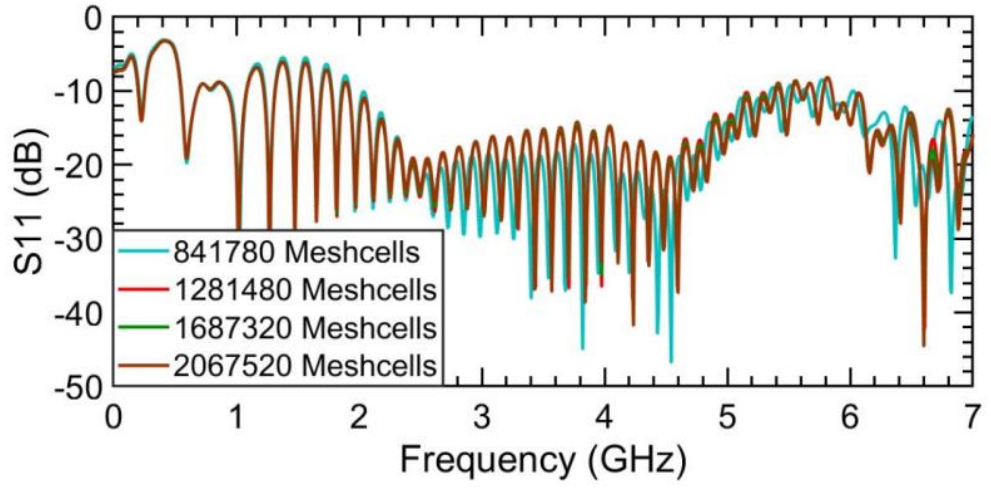

Figure 4.10: Comparison of the S11 of the SWS with number of mesh cells.

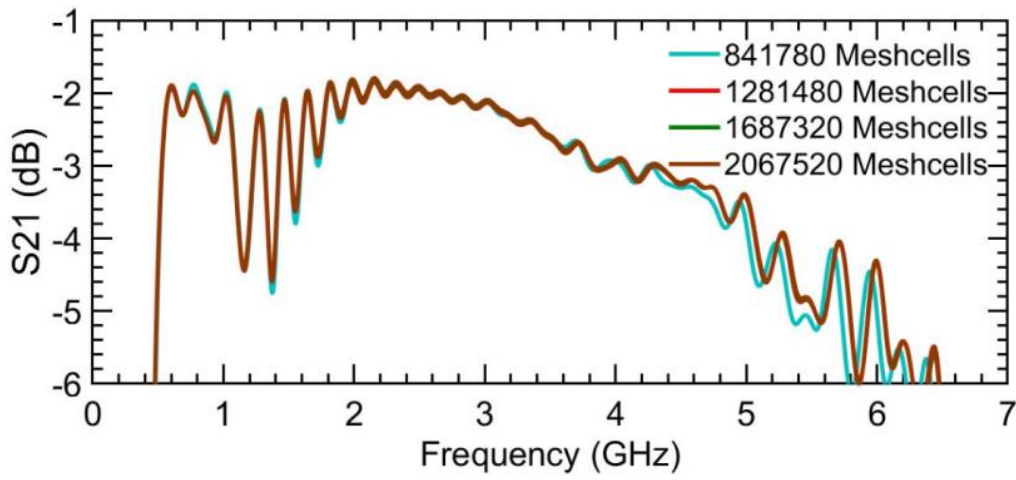

Figure 4.11: Comparison of the S21 of the SWS with number of mesh cells.

The S11 and S21 of the 30-period long SWS with different mesh size are shown in Figs. $4.10-4.11$, respectively. These figures show the convergence of the S-parameters of the SWS with mesh size. From Fig. 4.10, we can make out that the difference in the values of $\mathrm{S} 11$ at $3.5 \mathrm{GHz}$ for 1687320 and 2067520 mesh cells is $0.8 \%$. Similarly, Fig. 4.11 shows that the difference in the values of S21 at $3.5 \mathrm{GHz}$ for 1687320 and 2067520 mesh cells is $0.75 \%$.

We conclude that 2067520 mesh cells yield a converged result for the S-parameters of the SWS. This is the number of mesh cells used for the results reported in this chapter.

\subsubsection{COMPARISON OF S-PARAMETERS}

The measured S-parameters of the SWS are compared with those from simulations. As shown in Fig. 4.12, the simulated and measured S21 match very well over the frequency range $2 \mathrm{GHz}$ to $5 \mathrm{GHz}$, which is the targeted operation range of the SWS. The measured S21 is slightly lower than the simulated one. This is attributed to the fabrication tolerance and soldering of SMA connectors in the fabricated SWS. Also, the simulations do not 
include the small loss of the SMA connectors. Another reason for the mismatch is the difference between simulated straight edge connections and fabricated plated through holes (PTH). Between 2-5 GHz, the simulated $\mathrm{S} 11$ values are better than $-15 \mathrm{~dB}$ while the measured $\mathrm{S} 11$ values are better than $-12.5 \mathrm{~dB}$.

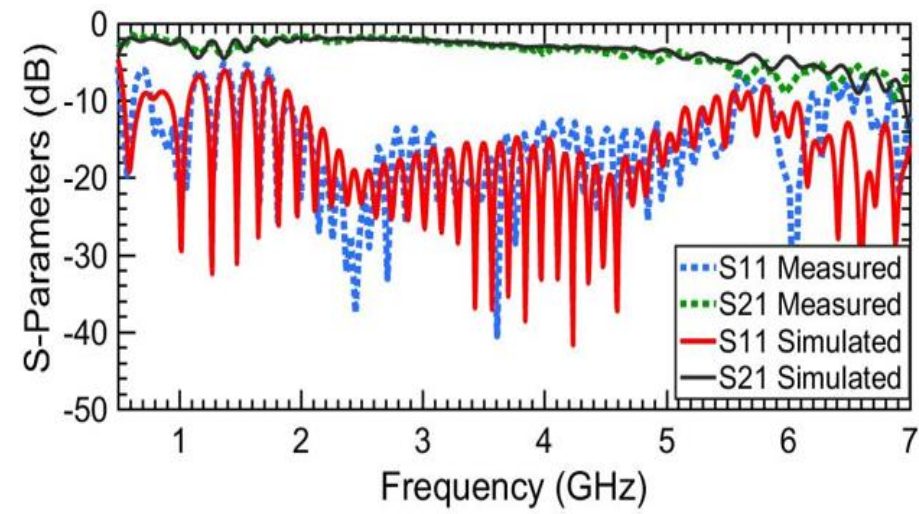

Figure 4.12: Comparison of measured and simulated S-parameters of SWS with 30 periods.

Regarding the deviation between measured and simulated S-parameter values above $5.5 \mathrm{GHz}$, it should be noted that the stop-band of the periodic structure starts around this frequency (see Fig. 4.8). There occur significant changes in the values of S-parameters around the stop-band. Since due to the fabrication tolerances there is usually a slight mismatch between the stop-band of the fabricated structure and the simulated one, the measured and simulated S-parameter values appear to have more deviation around the stopband.

\subsubsection{REGULARLY SPACED DIPS IN S11 RESULTS}

Fig. 4.15 shows regularly spaced dips in S11 results. Typically, such dips occur due to two relatively dominant mismatches of comparable strength, for example at the input and output of the SWS. The reflections caused by these mismatches interfere at port 1 , constructively or destructively as the frequency changes. In such a case, the frequency spacing of the dips is the reciprocal of the time delay between the two reflected signals arriving at port 1. The following section illustrates that the regular dips in Fig. 4.12 also satisfy the abovementioned relation.

Consider the simulation model of SWS shown in Fig. 4.13. This SWS is modelled in Transient solver with open boundaries. The simulated results in Fig. 4.12 are obtained 
from this model. The left port of this SWS is considered as port-1 while the right port is considered as port- 2 .

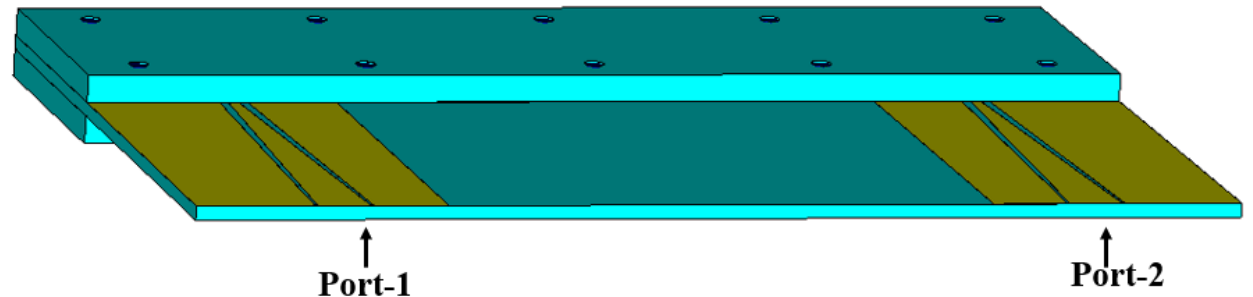

Figure 4.13: Perspective view of the SWS with 30 periods used in Transient mode solver.

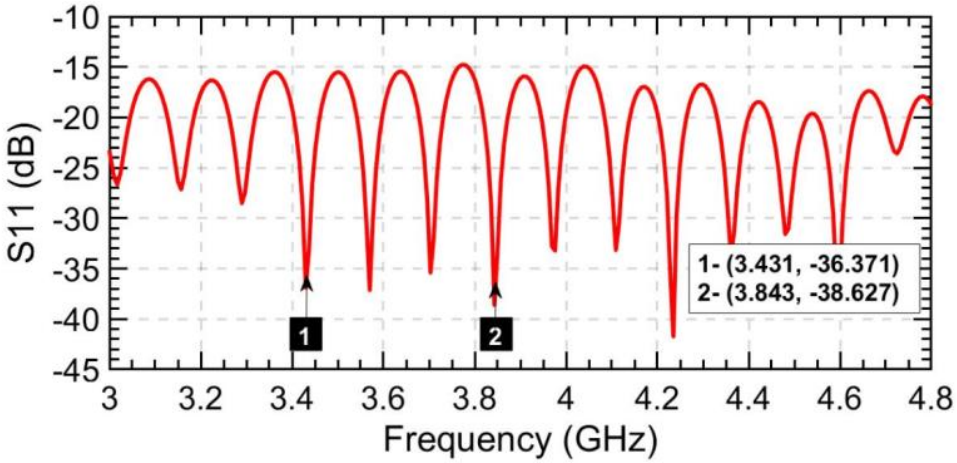

Figure 4.14: Zoomed version of S11 of the SWS.

Fig. 4.14 shows a zoomed version of the simulated S11 plot. A closer observation of the figure shows that the dips in the S11 plot do not have a constant frequency spacing. We consider the frequency spacing between three dips around $3.5 \mathrm{GHz}$ which is $412 \mathrm{MHz}$, giving the frequency spacing between adjacent dips as $\sim 137.3 \mathrm{MHz}$.

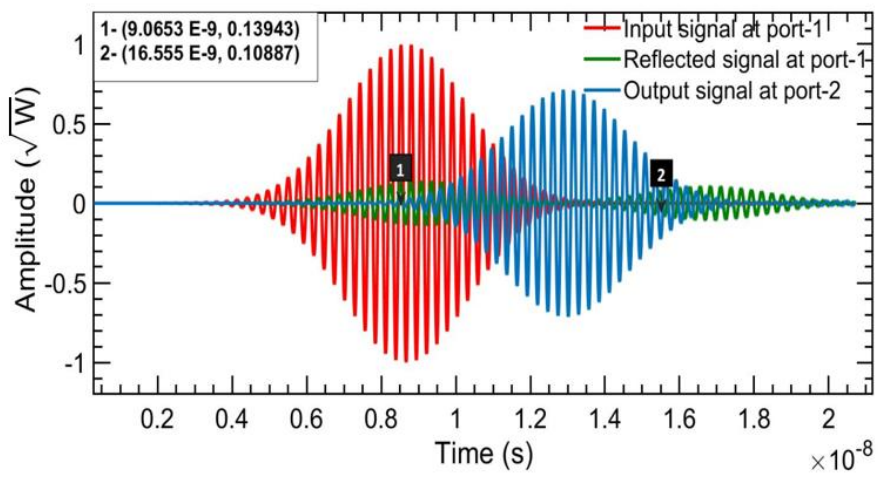

Figure 4.15: Input and output signals from Transient solver. The red curve represents the input signal applied at port-1, the blue curve represents the output signal obtained at port2 , and the green curve represents the reflected signal obtained at port-1. 


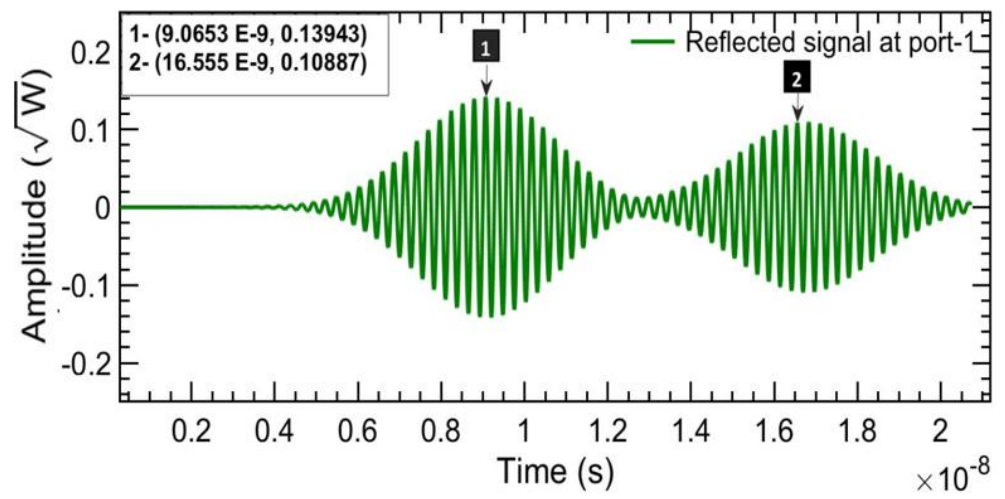

Figure 4.16: A zoomed version of the reflected signal obtained at port-1.

For obtaining the transit time for signals in the SWS, we have simulated the structure in CST Transient solver for the frequency range $3.43 \mathrm{GHz}$ to $3.84 \mathrm{GHz}$. Time variation of the input and output signal from CST Transient solver is shown in Fig. 4.15. The figure shows three signals: the applied input signal at port-1, output signal from port-2, and the reflected signal at port-1.

A zoomed version of the reflected signal at port-1 is shown in Fig. 4.16. The reflected signal at port -1 has two main components. The first one is due to the reflection of a part of input signal at port-1. The second component is due to the reflection of a part of the signal reaching port-2. Time delay between these two components is obtained from Fig. 4.16 as $7.4897 \mathrm{~ns}$. This is the time delay between the arrival of the two components of the reflected signal at port-1. This time delay corresponds to a frequency of $133.5 \mathrm{MHz}$. This value is quite close to the frequency spacing between adjacent dips.

\subsubsection{COMPARISON OF PHASE VELOCITY}

The phase constant of the PH-SEC is measured by measuring the phase of S21 values of the SWSs with two different lengths. The phase constant $\beta$ of the PH-SEC is obtained as:

$$
\beta=\frac{\Delta \angle S 21}{\Delta Z}
$$

where $\triangle \angle S 21$ is the difference in the phase of S21 of the SWSs with 30 periods and 25 periods of PH-SEC. $\triangle Z$ is the difference in length of SWSs, i.e., the length of 5 periods of the PH-SEC. The measured phase constant is used to calculate the phase velocity of the SWS. This value of phase velocity is compared with the results from tape-helix analysis and simulation in Fig. 4.17. As shown in the figure, the measured phase velocity values 
have a good match with those from the analysis. Besides, the measured values of phase velocity closely match with the simulation results. The slight deviation between the phase velocity from analysis and from measurement may be coming from the finite thickness of the top and bottom dielectric substrates in the fabricated SWSs. The analysis considers a homogeneous medium extending to infinity. These results provide an experimental validation of the tape-helix analysis of PH-SEC.

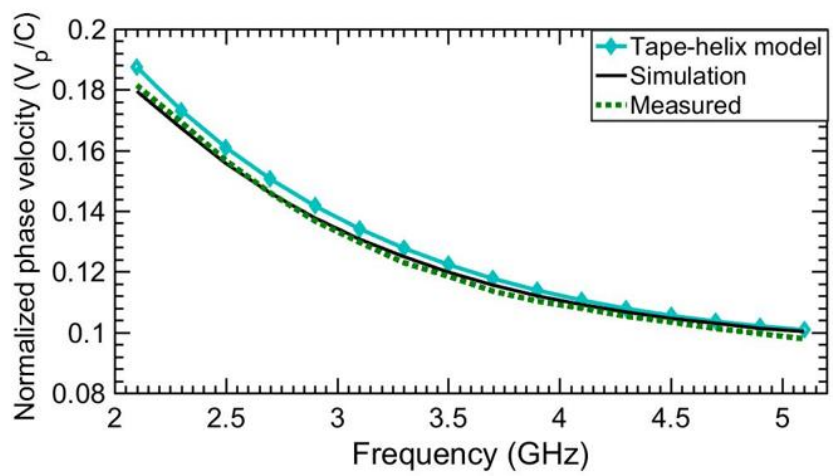

Figure 4.17: Comparison of normalized phase velocity of the PH-SEC.

\subsubsection{Comparison with Tape-Helix Analysis of Rectangular Helix [86]}

The tape-helix analysis of a rectangular tape-helix SWS in [86] applies the modified Marcatili's method and average power flow matching at boundaries to derive the characteristic equation. The analysis considers the confinement of the rectangular helix in both $x$ - and $y$-directions in a single step, which leads to a complex analysis and rather complicated characteristic equation.

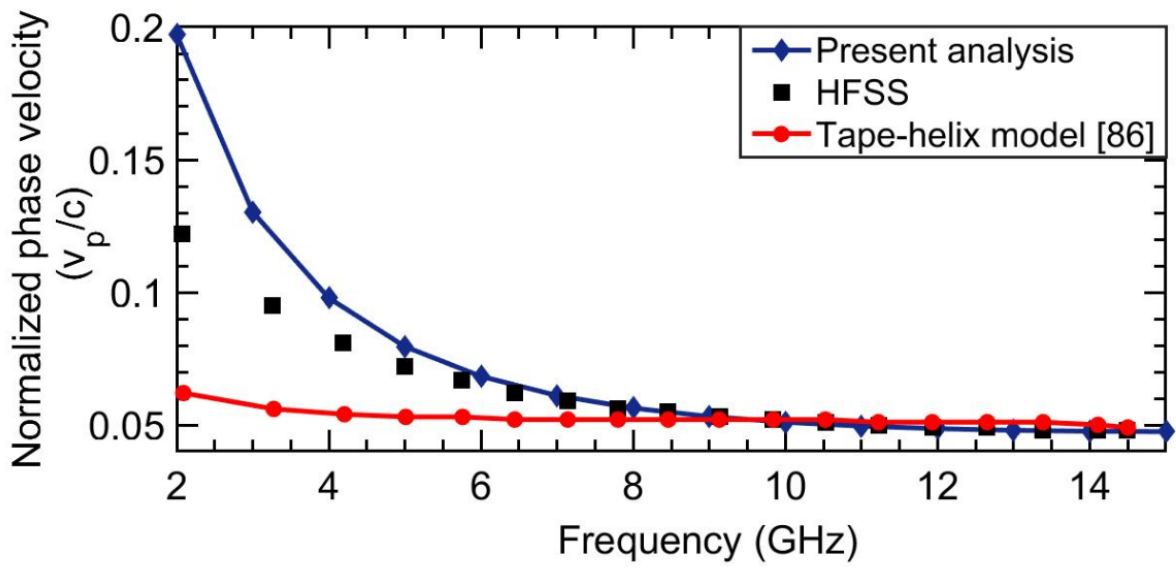

Figure 4.18: Comparison of normalized phase velocity for a rectangular tape helix with

$$
b / a=10, L / a=2, w / L=0.2 \text { and } a=0.232 \mathrm{~mm} \text {. }
$$


The tape-helix analysis presented in the previous section is simpler on account of using the EDC method. This analysis is used to evaluate the dispersion characteristics of rectangular helix SWS. While finding the dispersion characteristics of the rectangular helix, the effective pitch angle of PH-SEC is replaced by the actual pitch angle of rectangular helix.

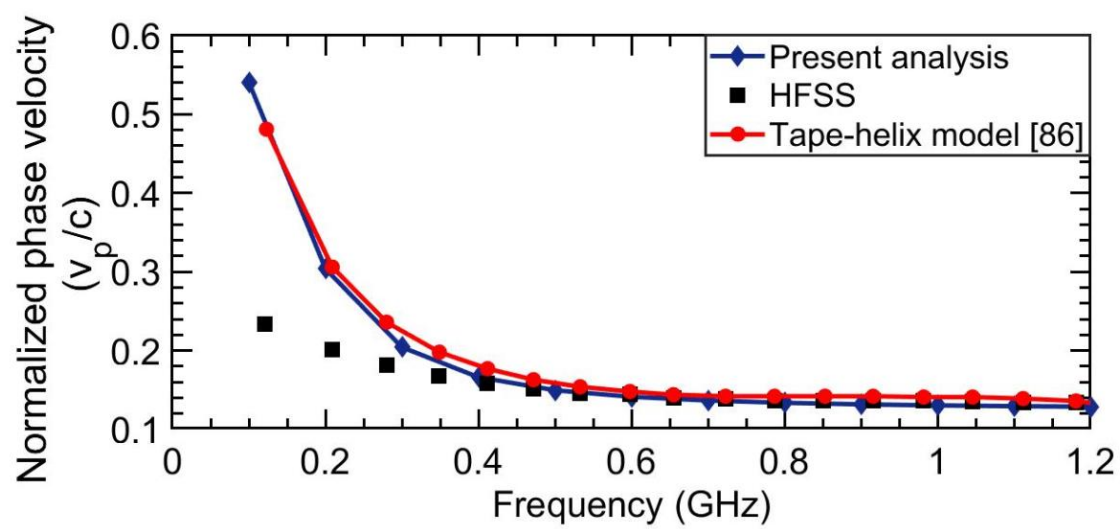

Figure 4.19: Comparison of normalized phase velocity for a rectangular tape-helix with

$$
b / a=1, L / a=1, w / L=0.1 \text { and } a=15.85 \mathrm{~mm} .
$$

The normalized phase velocity from the two analyses for a rectangular tape-helix with $b / a=10, L / a=2, w / L=0.2$, and $a=0.232 \mathrm{~mm}$ is shown in Fig. 4.18. The figure also includes the results from HFSS simulation which are reported in [86] for the same helix. The comparison reveals that the results from our analysis have a better match with HFSS simulation results over a wide frequency range.

The results of the normalized phase velocity from the two analyses for a rectangular tape-helix with $b / a=1, L / a=1, w / L=0.1$, and $a=15.85 \mathrm{~mm}$ are shown in Fig. 4.19. This figure also includes the HFSS simulation results reported in [86]. This figure also shows that the results from our analysis have a slightly better match with the results from HFSS simulation at relatively high frequencies as compared to those reported in [86].

\subsubsection{Comparison with Tape-Helix Analysis in [101]}

The first attempt to obtain the characteristic equation for the infinitely wide planar helix using tape-helix approximation was reported in [101]. The analysis in [101] considered different space harmonic distributions in both y-and z-directions, which leads to complex field expressions. The characteristic equation in [101] is derived using the variational approach. In our work, the analysis is simplified by assuming similar space harmonic distribution in both $\mathrm{y}$ - and z-directions. In addition, the analysis is further 
simplified by deriving the characteristic equation by simply equating the electric field parallel to the strips to zero.

In the tape-helix analysis of PH-SEC presented in Section 4.2 the characteristic equation of an infinitely wide planar helix from the tape-helix analysis is used to obtain $\beta_{A}$ from the $x$-dependent profile of the EDC method. In this sub-section, we use the characteristic equation from [101] to obtain the dispersion characteristics of PH-SEC. Thus, in place of (4.15), the characteristic equation from [101] is used in the $x$-dependent profile of the EDC method. The result from this analysis is compared with the results from our work.

\subsubsection{Characteristic Equation for the X-Dependent Profile}

The characteristic equation for the infinitely wide planar helix from [101] is given as:

$$
\sum_{n=-\infty}^{+\infty}\left\{\frac{\left(j \omega \mu_{0}\right) u_{n} \cos ^{2} \psi}{k_{c}^{2}}\left(\frac{J w_{z}}{p_{z}} \operatorname{sinc}\left(\frac{\beta_{n} w_{z}}{2}\right)\right)\left[1-\frac{1}{u_{n}^{2} k_{o}^{2}}\left(\frac{2 \pi n}{p_{y}} \beta_{n}+k_{c}^{2} \tan \psi\right)^{2}\right]\right\}=0
$$

All parameters in the above equation have the same meaning and expression as explained in Section 4.2. Equation (4.25) has a slight difference from the characteristic equation in (4.15). This difference is due to the difference in the assumptions and the conditions for deriving the characteristic equation in both analyses.

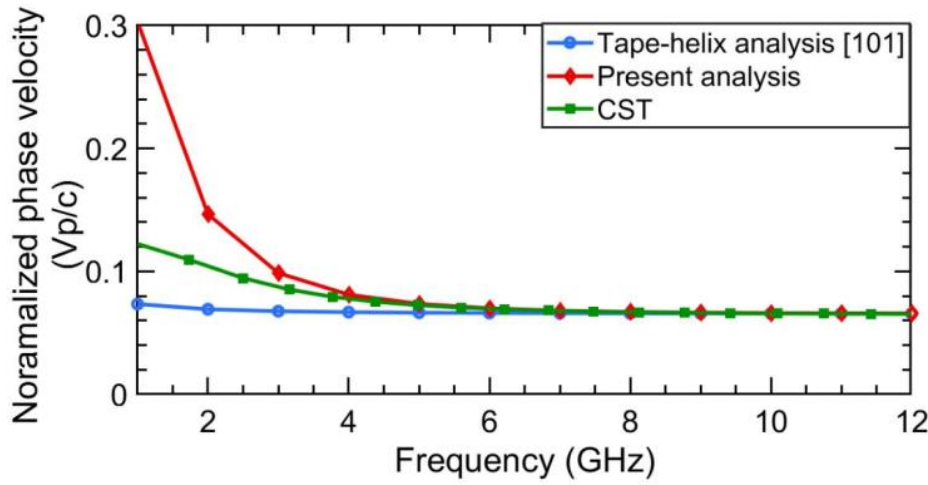

Figure 4.20: Comparison of normalized phase velocity of a PH-SEC with $b / a=4, w / L=$ 0.2 , and $a=0.75 \mathrm{~mm}$.

\subsubsection{Comparison With the Present Analysis}

The normalized phase velocity from simulation as well as the two analyses mentioned above for a PH-SEC with $b / a=4, w / L=0.2$, and $a=0.75 \mathrm{~mm}$ is shown in Fig. 4.20. 
Fig. 4.21 shows similar results for a PH-SEC with $b / a=1, w / L=0.2$, and $a=1.5 \mathrm{~mm}$. In both figures, the result from the analyses match very well and also show a good match with the CST simulation results except at relatively low frequencies. The mismatch at low frequency is due to the inaccuracy of the EDC method at these frequencies. These figures show that the present simplified analysis can give accurate results of dispersion characteristics of $\mathrm{PH}-$ SEC for a wider range of frequencies compared to the results obtained by using the characteristic equation in [101].

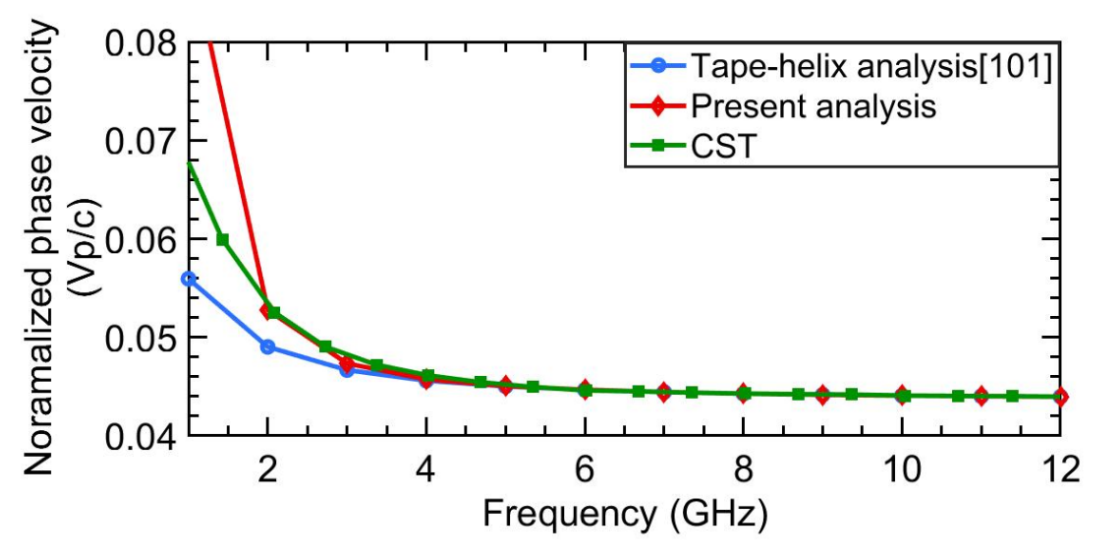

Figure 4.21: Comparison of normalized phase velocity of a PH-SEC with $b / a=1, w / L=$ 0.2 , and $a=1.5 \mathrm{~mm}$.

\subsection{Analysis of PH-SEC in a Metal Enclosure}

As an extension of the tape-helix analysis of the PH-SEC in a homogeneous medium in Section 4.2, the PH-SEC enclosed inside a metal enclosure is analysed in this section. As shown in Fig. 4.22, the metal enclosure has a separation $d$ from the PH-SEC in both $x$ and $y$-directions. The PH-SEC has transverse dimensions of $2 a$ along the $x$-direction and $2 b$ along the $y$-direction. $L$ is the period of the PH-SEC along the $z$-direction. The thickness of conductors in the PH-SEC is considered to be infinitesimally small in the analysis. The medium inside and outside the PH-SEC is considered as free space. Here also the analysis is simplified using the EDC method. The $x$-dependent profile in the EDC method considers an infinitely wide planar helix shielded by a pair of infinitely wide metal plates. Similar to the analysis of the PH-SEC immersed in a homogeneous medium (Section 4.2), the $y$ dependent profile considers an infinitely long dielectric slab which is confined only in the $y$-direction. 


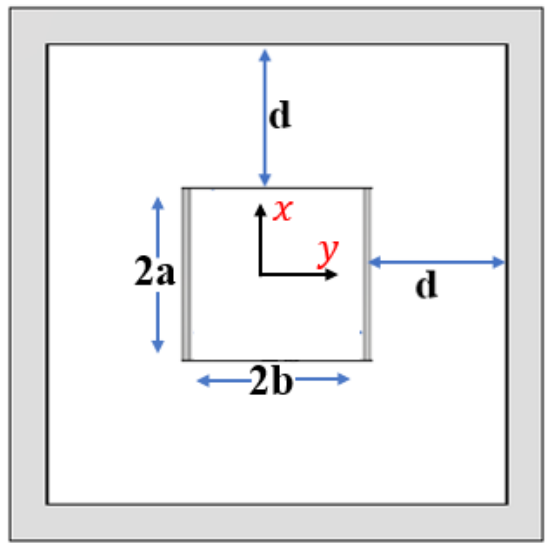

Figure 4.22: PH-SEC inside a metal enclosure.

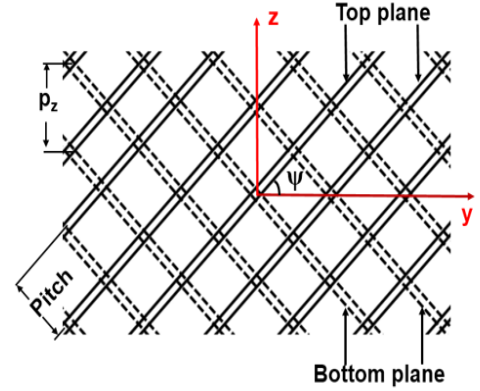

(a)

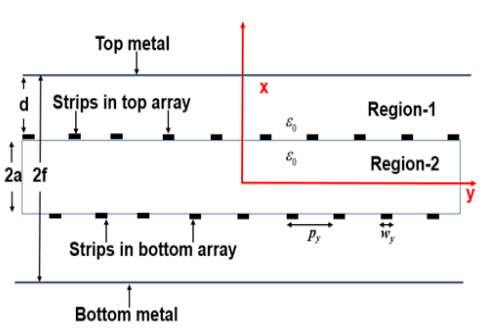

(b)

Figure 4.23: (a) Plan view (metal plates are hidden) and (b) cross-sectional view of the infinitely wide planar helix shielded by a pair of infinitely wide metal plates.

We can find the dispersion characteristics of the PH-SEC inside a metal enclosure by following all the steps of the EDC method presented in Section 4.2.2, except the characteristic equation used in the $x$-dependent profile. The $x$-dependent profile in section 4.2.2 uses the characteristic equation of an infinitely wide planar helix in a homogeneous medium, whereas here we have to use the characteristic equation of an infinitely wide planar helix shielded by a pair of infinitely wide metal plates. The following sub-sections present the various steps of this analysis, following the steps of Section 4.2. A comparison between the results from the analysis and simulation is also presented.

\subsubsection{Analysis of Infinitely Wide Planar Helix Shielded by Infinitely Long Metal}

\section{Plates}

An infinitely wide planar helix shielded by a pair of infinitely wide metal plates is shown in Fig. 4.23. The plan view of the structure without metal enclosure is shown in Fig. 4.23 (a), which is the same as the plan view of an infinitely wide planar helix in a 
homogeneous medium, i.e. Fig. 4.2 (a). Here also the strips of the infinitely wide planar helix are considered to be infinitely long in both $y$ - and $z$-directions. Therefore, all parameters in Fig. 4.23 (a) have similar meaning as explained in Section 4.2. The top and bottom conductor arrays are separated by a distance $2 a$ in the $x$-direction. As shown in Fig. 4.23 (b), a pair of infinitely wide metal plates are placed above and below the infinitely wide planar helix. The metal plates are infinitely wide in both $y$ - and $z$-directions, have a separation of $2 f$, and the distance between the metal plate and the infinitely wide planar helix is $d$.

\subsubsection{FIELD EXPRESSIONS}

The present structure also satisfies the four symmetry properties presented in Section 4.2.1. Hence it supports transverse symmetric and transverse-antisymmetric modes. For TWT application, we are interested in the latter type of modes. The expressions for the longitudinal electric and magnetic fields for the transverse-antisymmetric modes in the two regions are:

$$
\begin{aligned}
& E_{z 1}=\sum_{n=-\infty}^{\infty} A_{n} \sinh \left(u_{n}(f-x)\right) e^{\left\{j \frac{2 \pi n y}{p_{y}}\right\}} e^{\left\{-j \beta_{n} z\right\}} \\
& E_{z 2}=\sum_{n=-\infty}^{\infty} B_{n} \cosh \left(u_{n} x\right) e^{\left\{j \frac{2 \pi n y}{p_{y}}\right\}} e^{\left\{-j \beta_{n} z\right\}} \\
& H_{z 1}=\sum_{n=-\infty}^{\infty} C_{n} \cosh \left(u_{n}(f-x)\right) e^{\left\{j \frac{2 \pi n y}{p_{y}}\right\}} e^{\left\{-j \beta_{n} z\right\}} \\
& H_{z 2}=\sum_{n=-\infty}^{\infty} D_{n} \cosh \left(u_{n} x\right) e^{\left\{j \frac{2 \pi n y}{p_{y}}\right\}^{\left\{-j \beta_{n} z\right\}}}
\end{aligned}
$$

where subscripts 1 and 2 represent the field expression for region-1 (the region between planar helix and metal plate), and region-2 (the region inside planar helix), respectively. $\mathrm{n}$ is the number of space harmonic. All parameters such as $A_{n}, B_{n}, C_{n}, D_{n}, \beta_{n}$, $u_{n}$, etc. have similar meaning and expression as in Section 4.2.1. One can easily derive the expressions for the transverse field components from the expressions of longitudinal fields (Equation (4.26)) and the expressions given in Appendix C. The unknown amplitude constants $A_{n}, B_{n}$, $C_{n}$, and $D_{n}$ are solved using the same boundary conditions as in Section 4.2.1.

\subsubsection{CHARACTERISTIC EQUATION}

The characteristic equation for the infinitely wide planar helix shielded by a pair of infinitely wide metal plates is obtained using the condition $E_{1 \|}(x=a)=0$ and is given as: 


$$
\sum_{n=-\infty}^{+\infty}\left\{\begin{array}{c}
\left(j \omega \mu_{0}\right) \sinh \left(u_{n} d\right) \cos ^{2} \psi\left(\frac{J w_{z}}{p_{z}} \operatorname{sinc}\left(\frac{\beta_{n} w_{z}}{2}\right)\right) \\
{\left[\frac{u_{n}}{k_{c}^{2}} \frac{\sinh \left(u_{n} a\right)}{\sinh \left(u_{n} f\right)}-\frac{k_{c}^{2}}{k_{0}^{2} u_{n}^{2}} \frac{\cosh \left(u_{n} a\right)}{\cosh \left(u_{n} f\right)}\left(\frac{2 \pi n}{p_{y}} \frac{\beta_{n}}{k_{c}^{2}}+\tan \psi\right)^{2}\right]}
\end{array}\right\}=0 .
$$

The numerical solution of (4.27) gives a converged result by considering the first five to seven space harmonics.

\subsubsection{Application of the EDC Method}

The procedure for obtaining the dispersion characteristics of the PH-SEC using the EDC method has been explained in Section 4.2.2. Here, the $x$-dependent profile uses the characteristic equation (4.27). The effective pitch angle given in (4.16) is applied in (4.27) to obtain $\beta_{A}$. The effective dielectric constant for the $y$-dependent profile is obtained by substituting the value of $\beta_{A}$ in (4.17). The $y$-dependent profile explained in Section 4.2.2 can be directly applied to get the value of $\beta_{B}$. The final phase constant $\beta$ of the shielded $\mathrm{PH}-\mathrm{SEC}$ is obtained by the weighted averaging of $\beta_{A}$ and $\beta_{B}$, following (4.20).

The interaction impedance of the shielded PH-SEC is also obtained by following the procedure explained in Section 4.2.3.

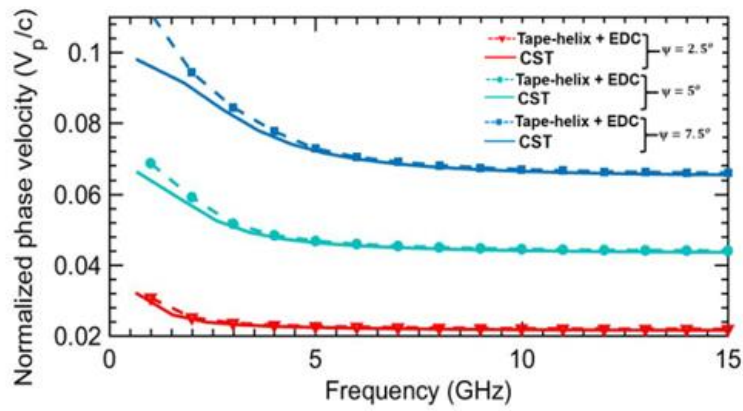

(a)

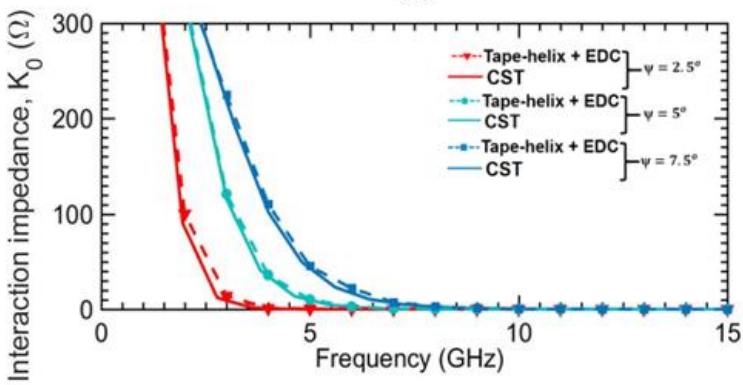

(b)

Figure 4.24: Comparison of simulation and analytical results for the shielded PH-SEC for three different pitch angles $\left(\psi=2.5^{\circ}, 5^{\circ}, 7.5^{\circ}\right)$ with $a / b=1, a=1.2 \mathrm{~mm}, d=2 \mathrm{~mm}$ and $w / L=0.2$. (a) Normalized phase velocity; (b) Interaction impedance. 


\subsubsection{Results and Discussion}

The normalized phase velocity and interaction impedance of the fundamental space harmonic for the shielded PH-SEC obtained from the above analysis are compared with the simulation results from CST eigenmode solver. Figs. 4.24 (a) and (b) show these results for the PH-SECs with $a / b=1, a=1.2 \mathrm{~mm}, d=2 \mathrm{~mm}$ and $w / L=0.2$. The figures show the results for three different pitch angles $\left(\psi=2.5^{\circ}, 5^{\circ}, 7.5^{\circ}\right)$. As shown in Figs. 4.24 (a), the normalized phase velocity from this analysis matches very well the simulation results for all three pitch angles for the frequency region far from cut-off. The phase velocity increases with increasing pitch angle. As shown in Figs. 4.24 (b), the results of interaction impedance of the shielded PH-SEC from this analysis also closely match those from simulation for all three pitch angles.

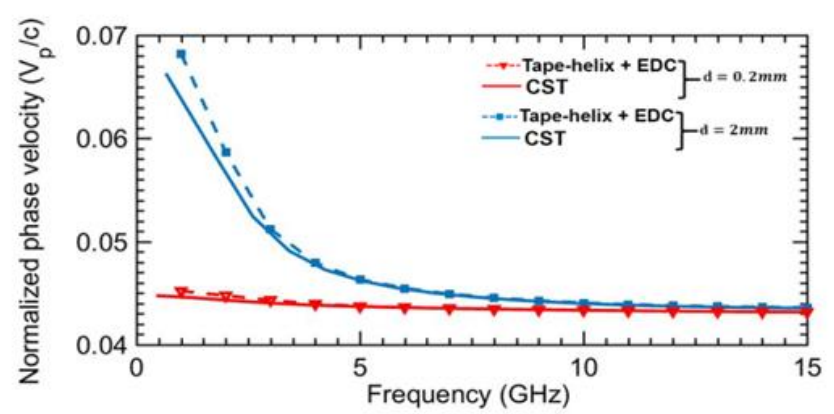

(a)

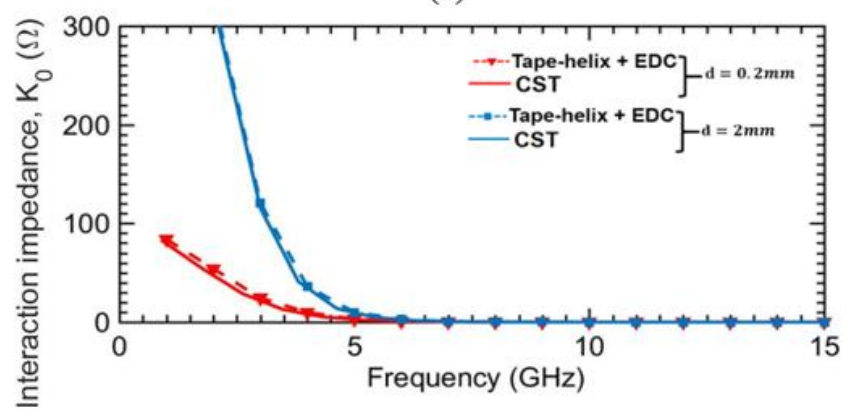

(b)

Figure 4.25: Comparison of simulated and analytical results for a PH-SEC inside a metal enclosure for two different size for metal enclosure $(d=0.2 \mathrm{~mm}, 2 \mathrm{~mm})$ with $a / b=1, a=$

$1.2 \mathrm{~mm}$, and $w / L=0.2$. (a) Normalized phase velocity; (b) Interaction impedance.

The normalized phase velocity and interaction impedance for the fundamental space harmonic of the shielded PH-SEC (with $a / b=1, a=1.2 \mathrm{~mm}$, and $w / L=0.2$ ) with two different enclosure sizes $(d=0.2 \mathrm{~mm}, 2 \mathrm{~mm})$ are presented in Figs. 4.25 (a) and (b), respectively. The normalized phase velocity shows a good match with the simulation results at a frequency region far from the cut-off for both enclosure sizes. The PH-SEC becomes 
less dispersive as the size of the metal enclosure decreases. As shown in Figs. 4.25 (b), the interaction impedance also closely matches the simulation results for both enclosure sizes. The interaction impedance of the shielded PH-SEC decreases as the size of the metal enclosure decreases.

These results show that the tape-helix analysis of the shielded PH-SEC can provide accurate results for the phase velocity and the interaction impedance of the fundamental space harmonic for a wide range of pitch angles and metal enclosure sizes.

\subsection{Summary}

In this chapter, field-theory based analysis to determine the dispersion characteristics as well as the interaction impedance of the fundamental space harmonic of the PH-SEC using the tape-helix approximation is presented. The analysis uses the characteristic equation of an infinitely wide planar helix together with the EDC method. The results from the analysis are compared with those from the simulation for PH-SECs with different aspect ratios and pitch angles. The comparison shows that the results from the present analysis closely match the results from simulation except at relatively low frequencies where the EDC method becomes less accurate. A comparison of the dispersion characteristics and interaction impedance of the PH-SEC from the present analysis and a previously reported analysis with sheath-helix approximation shows that the results from the present analysis are more accurate.

For an experimental validation of the tape-helix analysis of the PH-SEC, a PH-SEC SWS immersed in a homogeneous dielectric medium is designed and fabricated. The fabricated SWS operates over the frequency range from $2 \mathrm{GHz}$ to $5 \mathrm{GHz}$. The measured Sparameters of the SWS show a good match with those from simulations. The measured phase velocity shows an excellent match with that from simulations and a good match with that from the analysis.

The present analysis is also used for obtaining the dispersion characteristics of the rectangular tape-helix. These characteristics are compared with the HFSS simulation results and the tape-helix analysis of the rectangular helix reported in [86]. The comparison show that the results from the present analysis are more accurate over a broader bandwidth than the results from the more complicated analysis in [86]. 
The dispersion characteristics of the PH-SEC are also obtained using the characteristic equation of an infinitely wide planar helix reported in [101]. The results obtained in this manner are shown to agree very well with the simulation results and the results from the present analysis at frequencies far from the cut-off region.

As an extension of the tape-helix analysis of the PH-SEC in a homogeneous medium, the shielded PH-SEC is also analysed. Here also the results from the analysis agree very well with the simulation results at frequencies far from the cut-off region. 


\section{Chapter 5 A W-Band Backward-Wave Oscillator}

\subsection{Introduction}

The properties of the microfabrication-compatible planar helix slow-wave structure with straight-edge connections (PH-SEC) have been extensively studied for the development of wide band amplifiers. A wideband travelling-wave tube amplifier (TWTA) operating at Ka-band has been designed using the PH-SEC [32]. A sheet beam electronoptical system for a W-band (75-110 GHz) PH-SEC has been reported in [33]. This work shows that all components except the magnetic circuit of a PH-SEC-based W-band TWTA can be realized using microfabrication techniques. But the application of PH-SEC for backward-wave oscillators (BWOs) has not been studied yet. Investigation of backwardwave oscillation in the PH-SEC is useful for the development of microfabricationcompatible BWOs. In this context, the design of a W-band BWO with PH-SEC as the SWS is presented in this chapter.

The $\mathrm{W}$-band frequencies have a wide range of applications in communications, radar, spectroscopy, etc. BWOs and TWTAs are generally used for the generation and amplification of electromagnetic (EM) signals at these frequencies. Fabrication of travelling-wave tubes (TWTs) at such high frequencies using circular helix is challenging; hence microfabrication-compatible SWSs are required.

The rest of this chapter is organized as follows. The design of the PH-SEC to be used as the SWS for the W-band BWO is described in Section 5.2. This section also contains the dispersion characteristics, interaction impedance and the transmission characteristics of the SWS. The sensitivity of the dispersion characteristics and the interaction impedance to three of the geometrical parameters of the SW is also included in this section. These three parameters are the ones that are likely to vary rather significantly during the microfabrication process. The output performance of the BWO estimated from the particle-in-cell (PIC) simulations is presented in Section 5.3. This section describes the output power, efficiency and the frequency tuning of the W-band BWO. Section 5.4 describes the design, fabrication and measurement of an X-band PH-SEC SWS. The measured results of this scaled version of PH-SEC are compared with the simulation results to show the accuracy of results from simulation. This chapter ends with a summary in Section 5.5. 
5.2 Design of PH-SEC-Based BWO

\subsubsection{Configuration of the PH-SEC SWS}

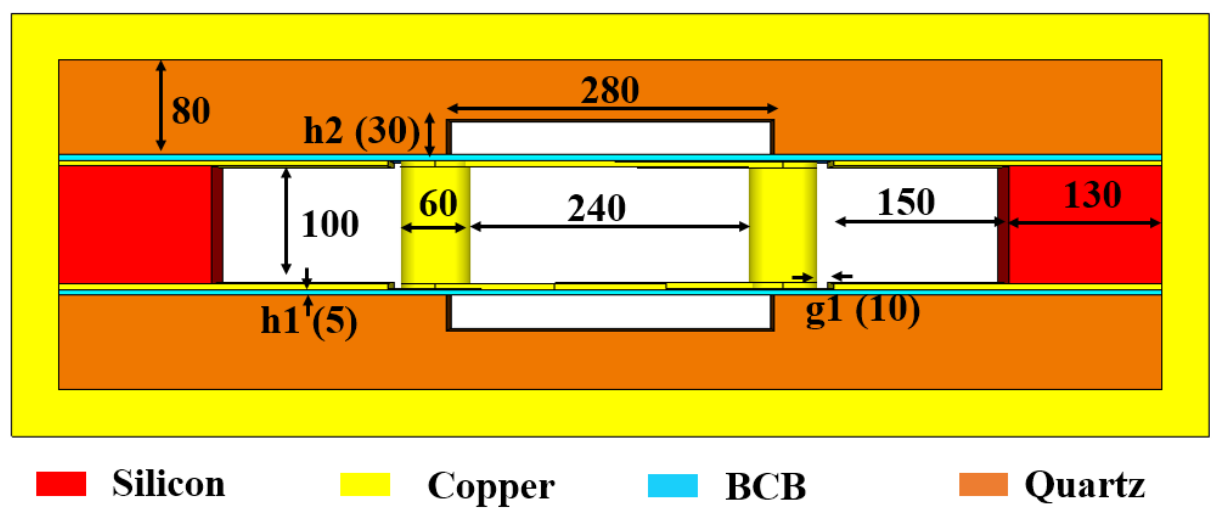

Figure 5.1: Cross-sectional view of the PH-SEC SWS with dimensions (all dimensions are in $\mu \mathrm{m})$.

The structure of microfabrication-compatible PH-SEC for the design of TWT amplifier in [104] is used for the design of BWO. The cross-sectional view of the SWS for $\mathrm{W}$-band BWO with dimensions of various geometrical features is shown in Fig. 5.1. The $\mathrm{PH}-\mathrm{SEC}$ is supported on quartz substrates inside a metal enclosure. The quartz substrates are assumed to have a dielectric constant of 4.43 and a loss tangent of $5.1 \times 10^{-5}$. Low loss nonconductive B-staged bisbenzocyclobutene $(\mathrm{BCB})$ layer with dielectric constant of 2.65 and thickness of $5 \mu \mathrm{m}$ is used as the bonding layer between the PH-SEC and the quartz substrates. Silicon with a dielectric constant of 11.9 and a loss tangent of $2.5 \times 10^{-4}$ is used as the spacer between the quartz substrates. As shown in the figure, four co-planar ground planes are placed close to the straight-edge connections of the PH-SEC. The PH-SEC, ground planes, and the metal enclosure are considered to be made of copper with an RF conductivity of $2 \times 10^{7} \mathrm{~S} / \mathrm{m}$. The pitch of the SWS is chosen as $375 \mu \mathrm{m}$ to enable operation of the BWO with a beam voltage around $10 \mathrm{KV}$.

Trenches are made in the quartz substrates to reduce the dielectric loading as well as dielectric charging [105]. The trenches help in increasing the interaction impedance of the SWS. Dielectric charging is the process of charge accumulation at the insulating parts of the SWS and may lead to dielectric breakdown. The charge accumulation occurs when the electrons hit the dielectric substrate due to insufficient strength of the focusing magnetic field or misalignment of the electron gun. This issue can be reduced by increasing the separation between the electron beam and the dielectric substrates. 


\subsubsection{Dispersion Characteristics}

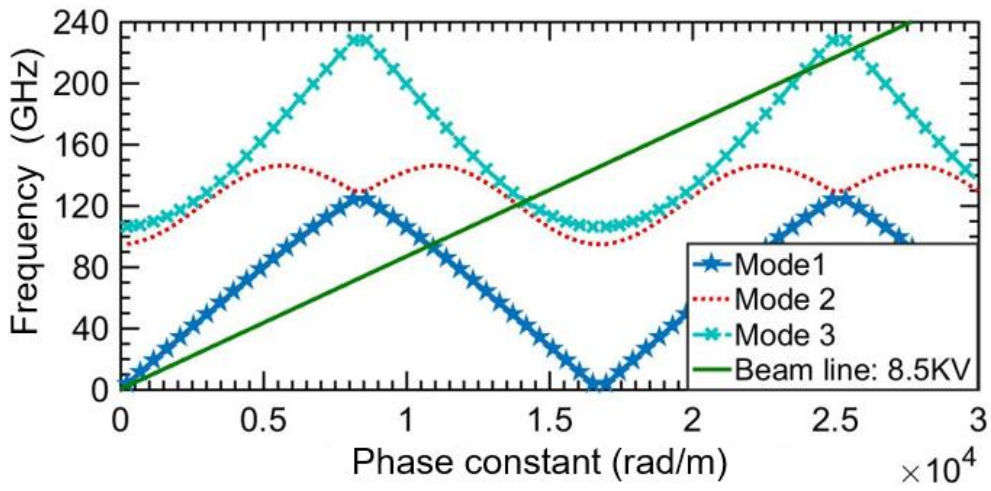

Figure 5.2: Dispersion characteristics of the SWS with beam line corresponding to 8.5 KV beam voltage.

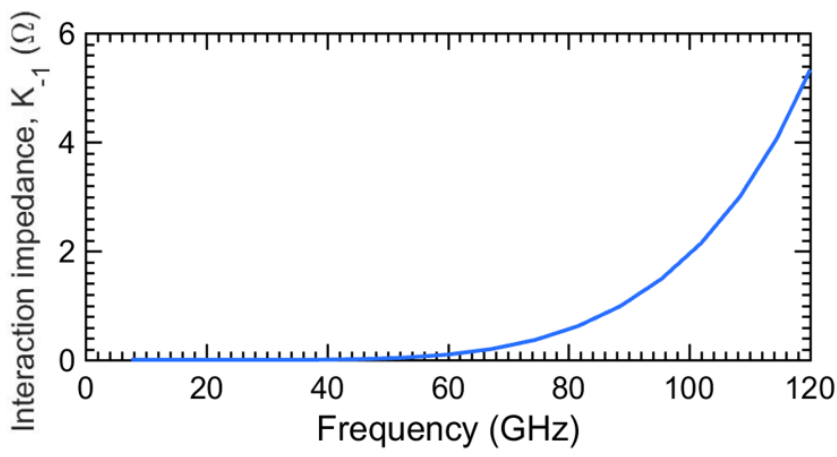

Figure 5.3: On-axis interaction impedance of $n=-1$ space harmonic of the SWS.

Eigenmode solver of Computer Simulation Technology (CST) is used for obtaining the dispersion characteristics of the SWS. The dispersion characteristics of the SWS together with the beam line corresponding to a beam voltage of $8.5 \mathrm{KV}$ are shown in Fig. 5.2. The dispersion characteristics contains three different modes of the PH-SEC. Mode-1 is used for the operation of BWO since it is the fundamental mode of the SWS. Mode-2 and mode-3 are the first and second higher order modes of the SWS, respectively. The interaction between the beam line and these higher order modes may cause unwanted oscillations in the BWO. As shown in the figure, the $8.5 \mathrm{KV}$ beam line intersects with the $\mathrm{n}=-1$ space harmonic of mode- 1 at $94 \mathrm{GHz}$, indicating the possibility of oscillation at a frequency around $94 \mathrm{GHz}$. This beam line also intersects with the backward-wave space harmonic of mode- 2 and mode- 3 . These intersections are an indication of the possibility of unwanted oscillation at frequencies around $120 \mathrm{GHz}$ and $225 \mathrm{GHz}$. A beam line for voltage higher (lower) than $8.5 \mathrm{KV}$ would intersect the $\mathrm{n}=-1$ space harmonic of the fundamental mode at frequencies higher (lower) than $94 \mathrm{GHz}$. 
The interaction impedance for the $\mathrm{n}=-1$ space harmonic of the fundamental mode of the SWS is shown in Fig. 5.3. The interaction impedance is evaluated using the Fourier decomposition method explained in Chapter 3. As expected, the interaction impedance of the $n=-1$ space harmonic increases with frequency, and a value of around $1.5 \Omega$ is indicated at $95 \mathrm{GHz}$.

\subsubsection{Sensitivity to Geometrical Parameters}

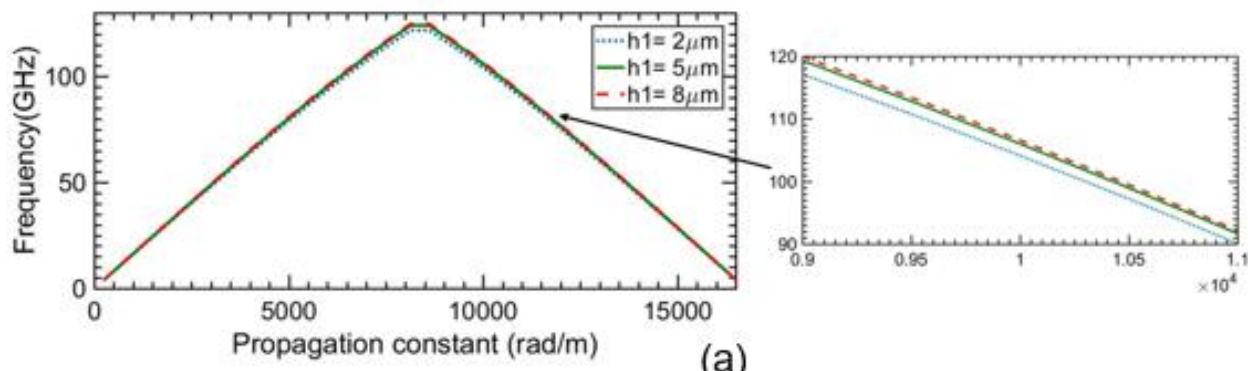

(a)
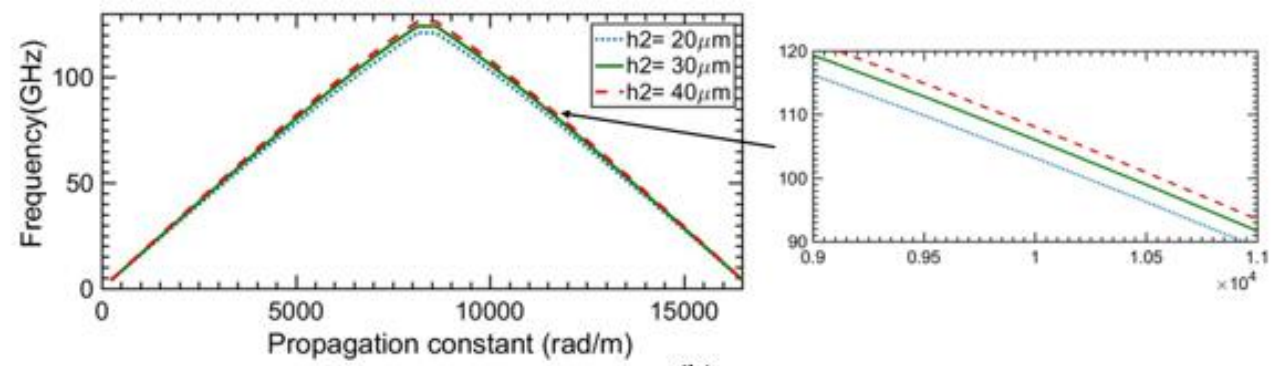

(b)
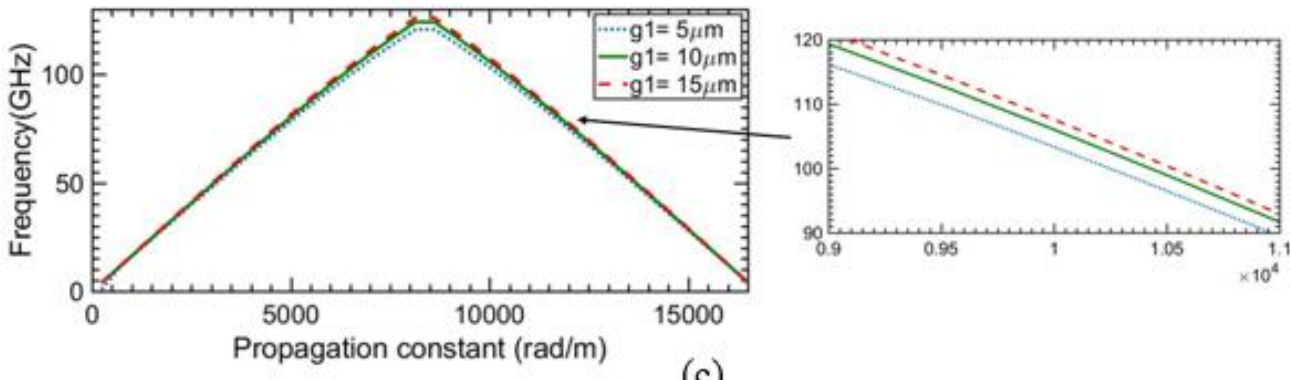

(c)

Figure 5.4: Dispersion characteristics with different values of (a) $h 1$, (b) $h 2$, and (c) $g l$. Zoomed in parts of the curves are also shown .

This section investigates the variation of dispersion characteristics and interaction impedance of the SWS due to fabrication tolerance. In the SWS shown in Fig. 5.1, the thickness of $\mathrm{BCB}(h 1)$, depth of the trench in the quartz substrates $(h 2)$, and the gap between PH-SEC and the ground planes $(g l)$ are the parameters more likely to vary during fabrication. Change in the phase constant of the fundamental and the $n=-1$ space harmonic of mode-1 of the SWS with variation of these geometrical parameters is presented in Figs. 
5.4 (a)-(c). As shown in Fig. 5.4 (a), the value of phase constant of the SWS for a given frequency increases slightly with increase in the thickness of BCB $(h 1)$. This reduces the slowing down factor for the EM wave in the SWS. Figs. 5.4 (b)-(c) show that the phase constant of the SWS for a given frequency increases also with increase in the value of geometrical parameters $h 2$, and $g 1$.

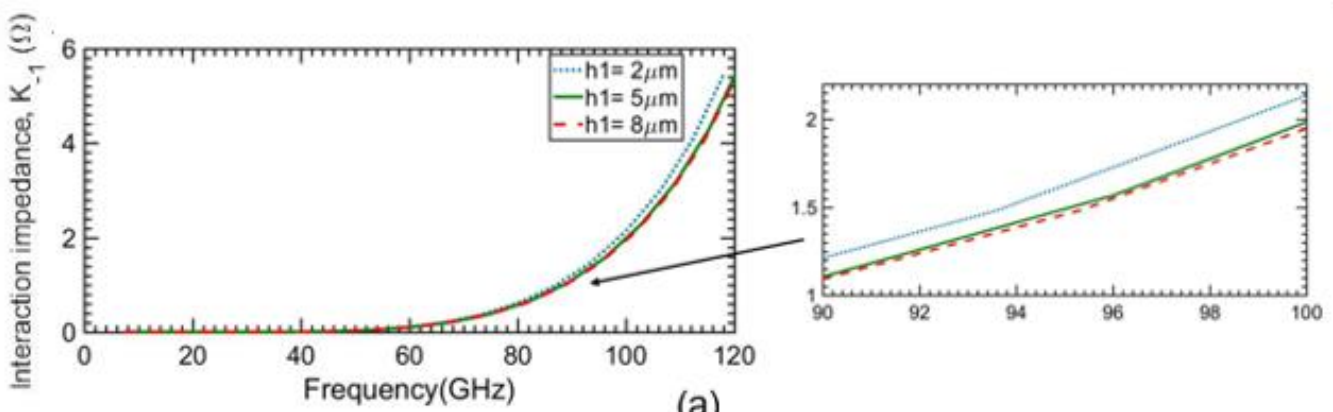

(a)

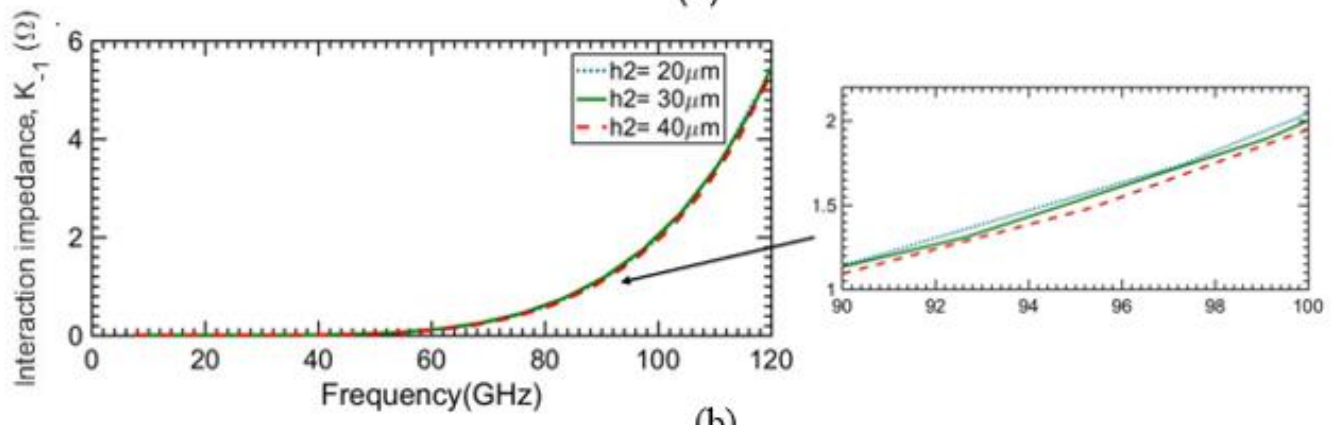

(b)

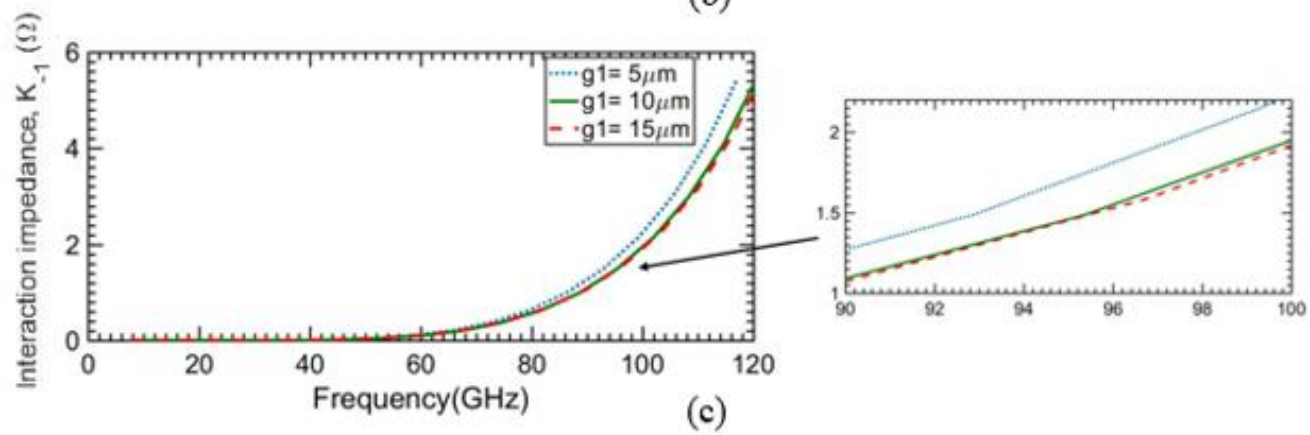

Figure 5.5: Variation of the interaction impedance with (a) $h 1$, (b) $h 2$, and (c) $g l$. Zoomed-in parts of the figures are also given.

Variation of the interaction impedance for the $n=-1$ space harmonic of the fundamental mode of the SWS with the three geometrical parameters is shown in Figs. 5.5 (a)-(c). The figures show that at a given frequency the interaction impedance of the $n=-1$ space harmonic increases as the value for the parameters $h l$ and $g l$ becomes smaller. Higher value of interaction impedance can increase the efficiency of the BWO. The parameter $h 2$ has negligible impact on the interaction impedance of the $n=-1$ space harmonic. Even though a smaller value for $h 1$ is more desirable for improving the 
performance of the BWO, we fix it as $5 \mu \mathrm{m}$ for easy realization using normal photolithographic process. Similarly, the value of $g l$ is fixed as $10 \mu \mathrm{m}$. Even though the interaction impedance is almost insensitive to the value of parameter $h 2$, a larger value of $h 2$ can help to reduce the phase velocity of the $n=-1$ space harmonic of the SWS. But the value of $h 2$ is fixed as $30 \mu \mathrm{m}$ since a larger value of $h 2$ reduces the mechanical stability of the quartz substrate.

\subsubsection{Transmission and Reflection Characteristics}

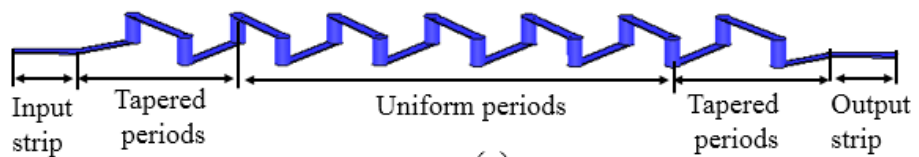

(a)

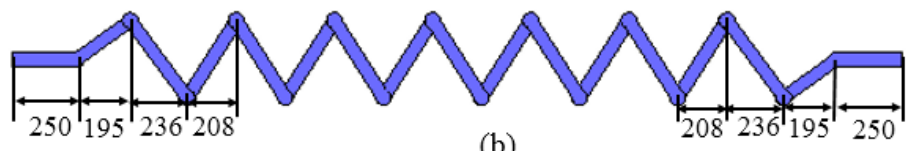

(b)

Figure 5.6: The PH-SEC SWS with 4 periods and stripline feeds. The metal enclosure and dielectric supports are not shown. (a) 3-D view, and (b) plan view with main dimensions (all dimensions are in $\mu \mathrm{m}$ ).

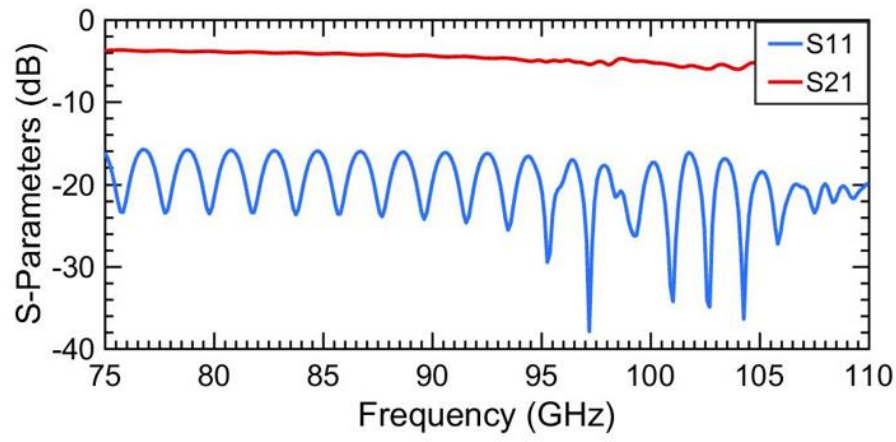

Figure 5.7: S-Parameters of the PH-SEC SWS for BWO.

The SWS for the BWO has sixty-two unit-cells of the PH-SEC shown in Fig. 5.1. Stripline feeds are connected at both ends of the SWS. Port-1 of the SWS (near the electron gun) is used for taking the output from the oscillator while port-2 is matched terminated. Pitch tapering is used at both ends of the SWS for getting a better transmission characteristic. Fig. 5.6 shows the stripline feeds and tapered periods for a PH-SEC SWS with four uniform periods. The figure also includes the dimensions of the tapered periods. The total length of the SWS is $24.78 \mathrm{~mm}$, which includes the sixty-two uniform periods, tapered periods and the stripline feeds. The horizontal strips of the PH-SEC and the stripline feeds can be 
fabricated together in the same process step since both are in the same plane. The transmission and reflection characteristics of the SWS, obtained from CST transient mode solver, are shown in Fig. 5.7. The $\mathrm{S} 21$ of the device is better than $-6.5 \mathrm{~dB}$ over the entire $\mathrm{W}$-band, while the device gives an $\mathrm{S} 11$ less than $-16 \mathrm{~dB}$ for this frequency range.

\subsection{PIC Simulation Results}

Once the 'cold' (in the absence of electron beam) design of the SWS is finalized using the Transient solver, the next step is to simulate the beam-wave interaction. This is done using particle-in-cell (PIC) solver which simulates the finalized 3D structure of the SWS together with an electron beam generated by a cathode. The shape of the cathode decides the shape of electron beam. This solver also is a time domain solver. It solves the Maxwell's equations together with force equations to simulate the interaction of the electromagnetic fields of the SWS with the electron beam. We can specify the time duration up to which the output of a device is desired. We can also specify the strength of the applied longitudinal magnetic field which keeps the electron beam from diverging. Hexahedral mesh cells are used for simulations. The number of mesh cells used in this solver is the same as the one that gives converged results in the Transient solver.

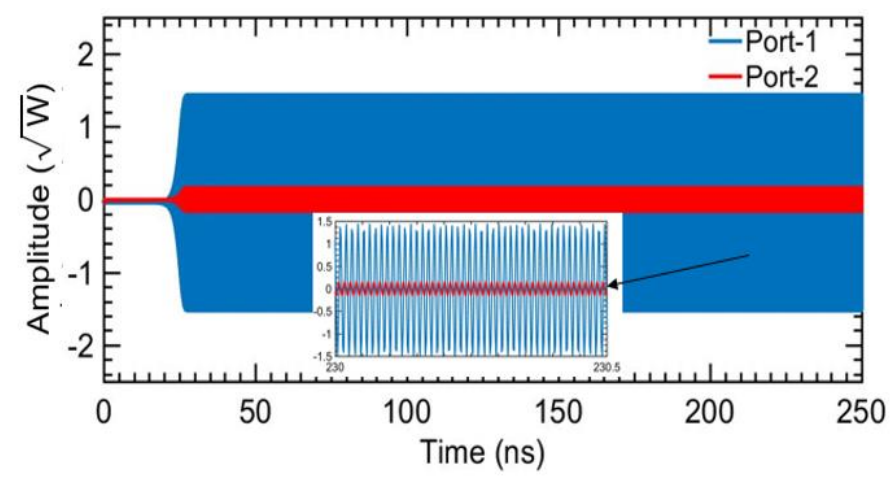

Figure 5.8: The output signal versus time of the BWO with $8.5 \mathrm{KV}$ beam voltage; zoomed version of signal is in the inset.

We have used 5628196 hexahedral mesh cells for the PIC simulation of BWO in this chapter. This number is obtained from the convergence study using the Transient solver. We have used a desktop with GPU acceleration (HP Z640) for PIC simulations. It took 10 days for completing the simulation of BWO for 250 ns with 5628196 hexahedral mesh cells.

The output characteristics of the BWO are simulated using the CST particle-in-cell (PIC) simulator. The characteristics of the BWO include output power, frequency of the 
output signal, DC-to-RF efficiency, and tuneable bandwidth. A sheet electron beam with cross-section dimensions of $160 \mu \mathrm{m} \times 40 \mu \mathrm{m}$ is used for the simulations. The beam current of the sheet beam is fixed as $20 \mathrm{~mA}$ and the beam voltage is varied from $7 \mathrm{KV}$ to $11 \mathrm{KV}$. The simulations use a magnetic field of $1 \mathrm{~T}$ to ensure confined flow of the electron beam without collision between the SWS and the electrons.

The time-domain output signal from the BWO for a beam voltage of $8.5 \mathrm{KV}$ is shown in Fig. 5.8. As shown in the figure, a strong output signal is generated at port-1, the port near cathode. The amplitude of the signal starts to grow at around $20 \mathrm{~ns}$ and attains a stable value at around $27 \mathrm{~ns}$. A signal with comparatively small amplitude is generated at the port near the collector, port-2. This signal is generated due to the reflection of the signal from port-1.

We have used MATLAB programme for finding the discrete Fourier transform (DFT) of the output signal from PIC solver. The used programme is given as Appendix D. The steps are briefly listed as follows:

- The time domain data of output signal from CST PIC solver is stored as a Text document (.txt).

- MATLAB command is used to read the document.

- The Text document has an array with two columns; the first column contains the time instants and the second column contains the corresponding data samples.

- We can identify the time instant of each data point with the help of the abovementioned array. For example, the data point at $0^{\text {th }}$ position is for $0 \mathrm{~ns}$, the data point at $30276^{\text {th }}$ position is for $50 \mathrm{~ns}$, etc.

- We select the required data points as input signal X.

- Consider, $\mathrm{X}=\mathrm{u}(0: 30276)$ command. This command creates $\mathrm{X}$ as an array with data points corresponding to time instant from $0 \mathrm{~ns}$ to $50 \mathrm{~ns}$.

- The length of $\mathrm{X}$ is used as the number of samples, $\mathrm{N}$

- We specify the sampling frequency, which is calculated from the sampling interval of the data points.

- We find discrete Fourier transform of X using FFT1=abs(fft(Inputsig1,N))./N; FFT1 gives the absolute value of normalized DFT.

- We select the right-hand side of FFT1 and plot the results as a function of frequency. 


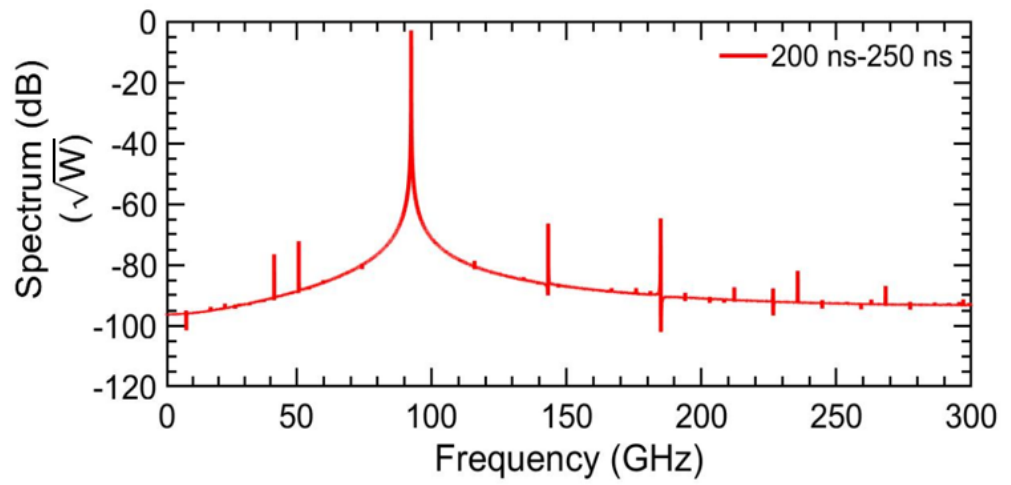

Figure 5.9: Discrete Fourier transform of BWO output at time window between 200 ns to $250 \mathrm{~ns}$

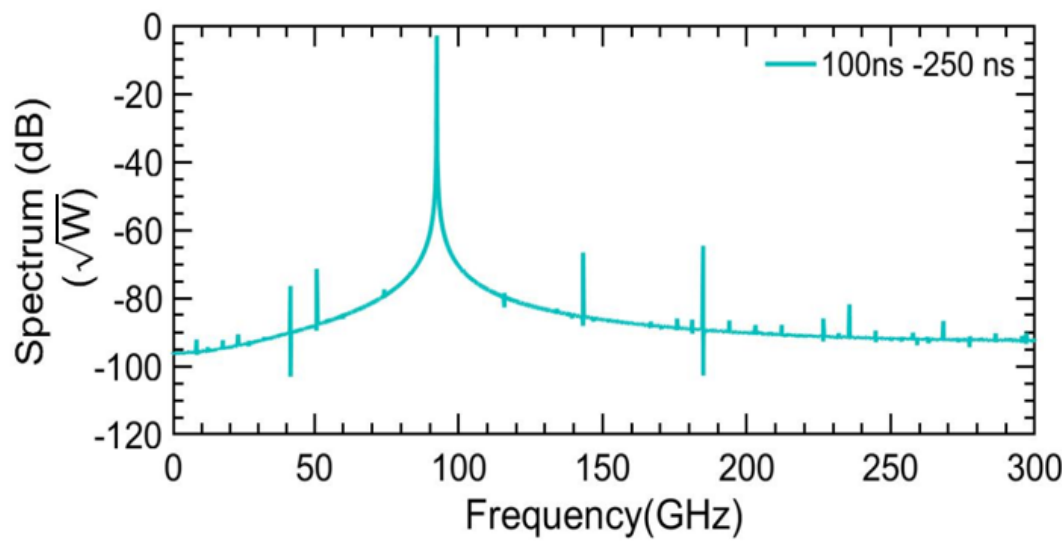

Figure 5.10: Discrete Fourier transform of BWO output at time window between $100 \mathrm{~ns}$ to $250 \mathrm{~ns}$

Using the steps given above, the spectrum of the output signal obtained from PIC simulations is shown in Fig. 5.9 and Fig. 5.10 for window sizes of $50 \mathrm{~ns}$ and $150 \mathrm{~ns}$, respectively.

The y-axis of Figs. 5.9-5.10 gives the strength of BWO output at each frequency. The PIC output shown in Fig. 5.8 has units of $\sqrt{W}$. Therefore, the input signal for discrete Fourier Transform has units of $\sqrt{W}$. Since the unit of y-axis after DFT is same as the unit of input signal for DFT, the units for y-axis in Fig. 5.9-5.10 should be $\sqrt{W}$.

The main peak in the spectrum is at $92.46 \mathrm{GHz}$, which shows that the BWO at 8.5 $\mathrm{KV}$ beam voltage gives an output signal at $92.46 \mathrm{GHz}$. This frequency is very close to 94 $\mathrm{GHz}$ indicated in the dispersion characteristics (Mode 1) in Fig. 5.2. A slight shift in the frequency with respect to the dispersion characteristics is due to loading of the SWS by the electron beam which has not been considered in the dispersion characteristics. Other than 
the desired signal at $92.46 \mathrm{GHz}$, the spectrum has some un-desired but much smaller peaks. Some of the prominent smaller peaks are around $41.7 \mathrm{GHz}, 50.7 \mathrm{GHz}, 143.2 \mathrm{GHz}, 184.9$ $\mathrm{GHz}, 235.6 \mathrm{GHz}$ etc. The strength of these peaks is given in Table 5.1 in the decreasing order of strength.

Table 5.1 Strength of various peaks in the Spectrum

\begin{tabular}{|c|c|}
\hline Frequency $(\mathrm{GHz})$ & Spectrum $(\mathrm{dB})$ \\
\hline 92.46 & -2.85 \\
\hline 184.9 & -64.6 \\
\hline 143.2 & -66.2 \\
\hline 50.7 & -71.4 \\
\hline 41.7 & -76.5 \\
\hline 235.6 & -81.8 \\
\hline
\end{tabular}

The small peaks are understood as follows.

a) Interaction of beam line with higher order modes: The peak at $235.6 \mathrm{GHz}$ is generated due to the interaction between backward-wave part of Mode 3 and beam line. A slight shift in the frequency with respect to the dispersion characteristics is due to beam-loading of the SWS.

b) Harmonics of the fundamental frequency: $184.9 \mathrm{GHz}$ is the second harmonic of the fundamental frequency $92.46 \mathrm{GHz}$.

c) Inter modulation products:

$50.7 \mathrm{GHz}$ corresponds to the inter modulation between $235.6 \mathrm{GHz}$ and $184.9 \mathrm{GHz}$ 143.2 $\mathrm{GHz}$ corresponds to the inter modulation between $92.46 \mathrm{GHz}$ and $235.6 \mathrm{GHz}$ $41.7 \mathrm{GHz}$ corresponds to the inter modulation between $92.46 \mathrm{GHz}$ and $50.7 \mathrm{GHz}$

As explained above, the very low-level frequency peaks are un-desired or spurious signals correspond to interaction of beam line with higher order modes, harmonics, or intermodulation products. The simulation results obtained from PIC solver are not random. One will get an identical result for different runs of the simulator if one uses the same set of simulation parameters.

The simulated phase space energy of the electrons at $30 \mathrm{~ns}$ is shown as a function of the longitudinal position in Fig. 5.11. The plot shows that more electrons are decelerated than accelerated at the end of the SWS, which is an indication of energy transfer from the electron beam to the EM wave. 
The performance of the BWO with $16 \mathrm{~mA}$ and $18 \mathrm{~mA}$ beam currents is also estimated using PIC simulations. The simulations with $16 \mathrm{~mA}$ and $18 \mathrm{~mA}$ beam currents use the focusing magnetic fields of $0.75 \mathrm{~T}$ and $0.9 \mathrm{~T}$, respectively.

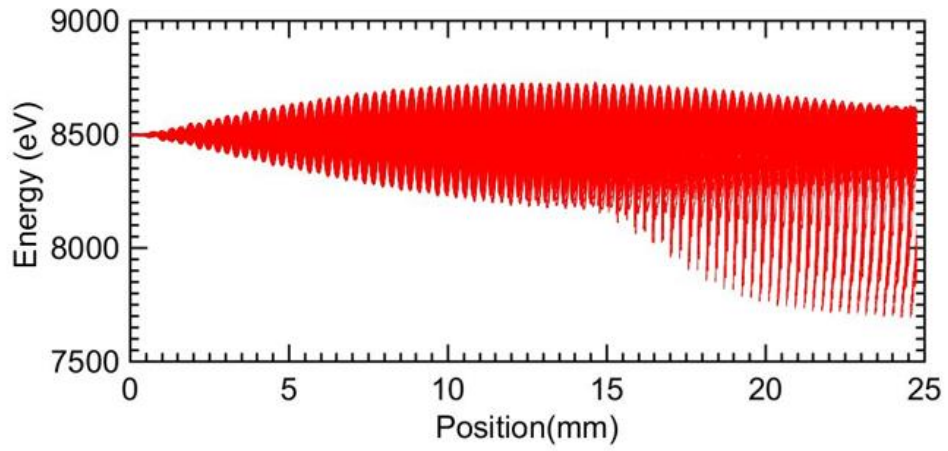

Figure 5.11: Phase space energy of the electrons vs. the longitudinal position at $30 \mathrm{~ns}$.

The frequency tuneability of the BWO for different beam voltages is shown in Fig. 5.12. As shown in the figure, the frequency of the output signal can be tuned from 86.9 $\mathrm{GHz}$ to $100 \mathrm{GHz}$ with a tuneable bandwidth of $14 \%$ around the centre frequency of 93.4 GHz. As indicated in the dispersion characteristics, the frequency of the output signal increases with increase in the beam voltage. The figure shows that the beam current has negligible effect on the frequency of the output signal from the BWO.

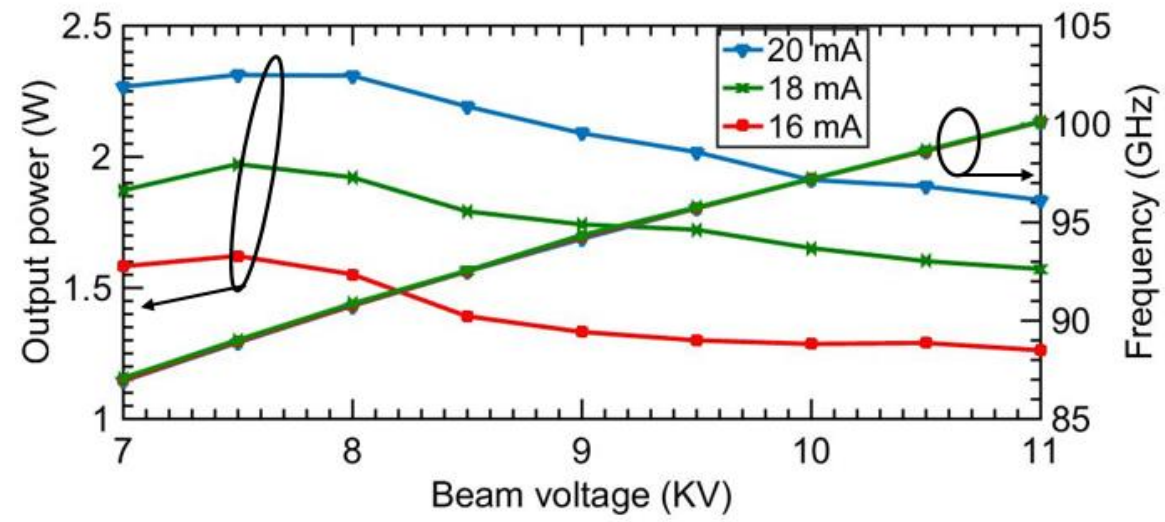

Figure 5.12: Variation of the output power and frequency with beam voltage for three different beam currents.

The Fig. 5.12 also presents the variation of the peak output power with frequency of the output signal (or beam voltage) for three different beam currents. The output power of the BWO for a given frequency increases with increase in the beam current. Maximum peak output power of $2.3 \mathrm{~W}$ at $88.8 \mathrm{GHz}$ is obtained for the beam voltage of $7.5 \mathrm{KV}$ and 
beam current of $20 \mathrm{~mA}$. This maximum peak output power drops to $1.97 \mathrm{~W}$ and $1.62 \mathrm{~W}$ as the beam current reduces to $18 \mathrm{~mA}$ and $16 \mathrm{~mA}$, respectively. Beam voltage of $11 \mathrm{KV}$ and beam current of $20 \mathrm{~mA}$ produce a signal with $1.83 \mathrm{~W}$ peak power at $100 \mathrm{GHz}$ frequency, which is the minimum peak power for this beam current.

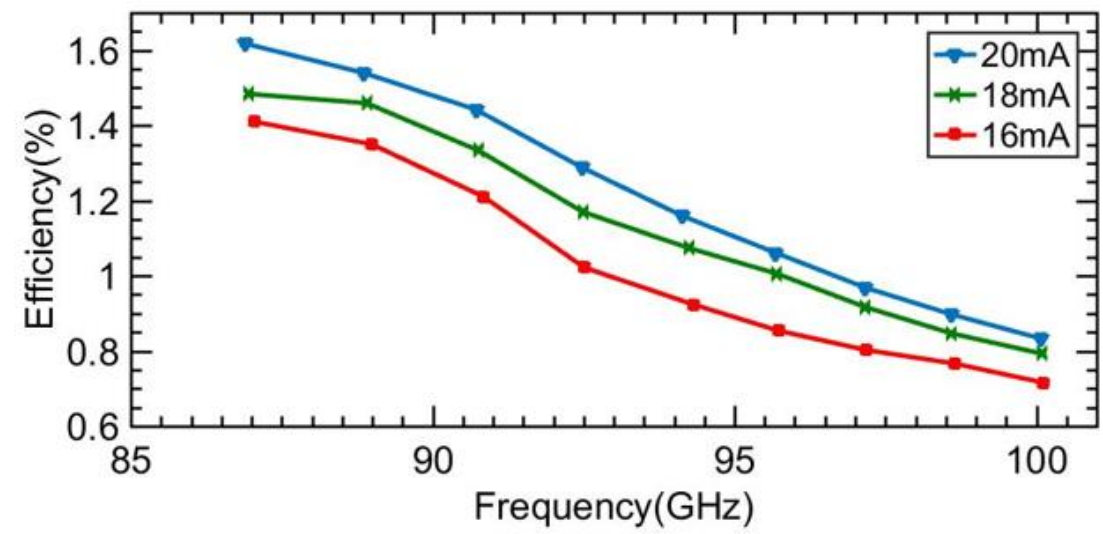

Figure 5.13: Peak DC-to-RF conversion efficiency of the BWO versus frequency for three different beam currents.

Variation of the DC-to-RF conversion efficiency of the oscillator with the frequency of oscillation for three different beam currents is shown in Fig. 5.13. The DC-to-RF conversion efficiency of the oscillator is defined as the ratio between the power of the output RF signal and the DC power of the electron beam:

$$
\eta=\frac{\text { Output RF power }}{\text { DC power of electron beam }} \times 100
$$

As shown in the figure, the DC-to-RF efficiency of the oscillator increases with beam current for a given output frequency. Besides, for a given beam current, the efficiency of the oscillator decreases with increase in frequency. The oscillator can provide a maximum DC-to-RF efficiency of $1.62 \%$; this drops to $1.48 \%$ and $1.41 \%$ for beam current of $18 \mathrm{~mA}$ and $16 \mathrm{~mA}$, respectively.

\subsection{Fabrication of PH-SEC}

A scaled version of the PH-SEC SWS is fabricated and measured to strengthen the confidence in simulation results described above. The scaled version of the PH-SEC is designed to operate at X-band and fabricated using printed circuit technique. This section presents the design, fabrication, and measurements of the scaled version. 


\subsubsection{Description of the Fabricated PH-SEC}

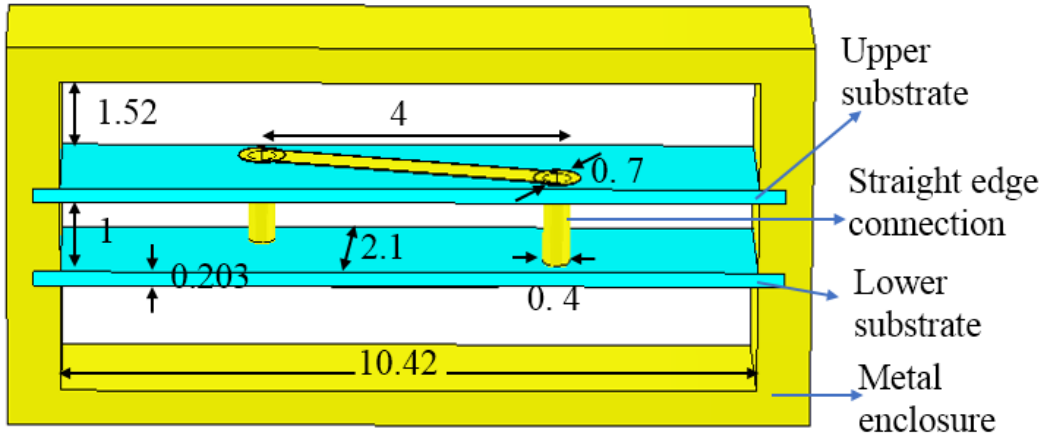

Figure 5.14: Structure and dimensions of the fabricated PH-SEC SWS (all dimensions are in millimetre).

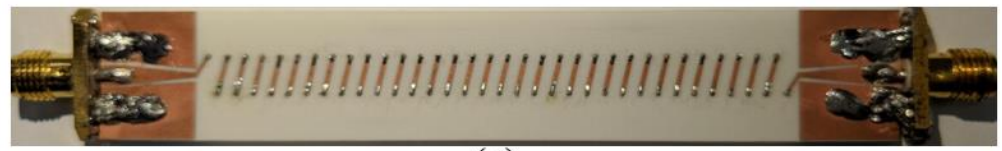

(a)

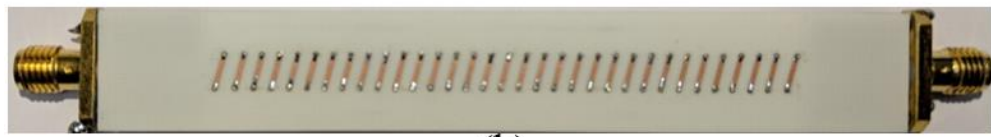

(b)

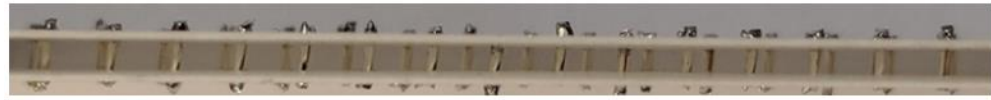

(c)

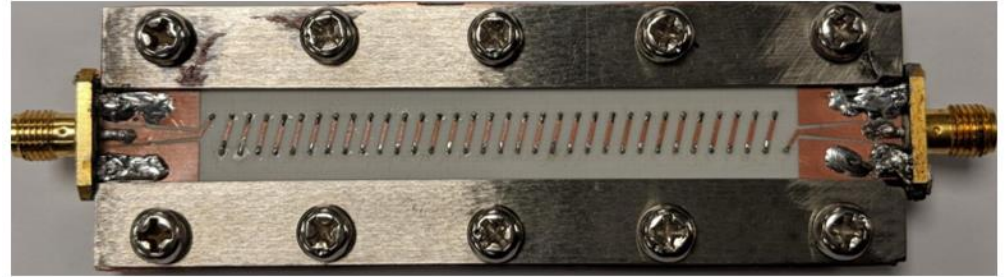

(d)

Figure 5.15: Prototype assembly of the SWS with 30 periods. (a) Top view. (b) Bottom view. (c) Side view. (d) SWS inside the metal enclosure (without the top cover of the metal enclosure).

Fig. 5.14 shows the structure and the dimensions of a single period of the scaled version of the PH-SEC. As shown in the figure, the PH-SEC is fabricated using two identical dielectric substrates. The top horizontal strips of the PH-SEC are fabricated on the first dielectric substrate while the bottom horizontal strips are realized on the second dielectric substrate. RO4003 substrates with thickness of $0.203 \mathrm{~mm}$, dielectric constant of 3.55 , and loss tangent of 0.0027 are used for the fabrication. The straight-edge connections 
between the horizontal strips of the PH-SEC are realized by soldering copper wires of 0.4 $\mathrm{mm}$ diameter. Circular pads of $0.7 \mathrm{~mm}$ diameter are designed at both ends of the horizontal strips for the ease of soldering the copper wires. The entire SWS is placed inside a metal enclosure. For the measurement of phase velocity of the PH-SEC, two identical SWSs with different number of periods, 25 and 30, are fabricated. For both SWSs, CPW feed-lines printed on the upper substrate are connected to SMA connectors. Pitch tapering is used at both ends of the SWS for getting a better match.

The photos of the assembled SWS with thirty periods are shown in Fig. 5.15. The upper substrate which contains the top horizontal strips of the PH-SEC and the CPW feed lines is shown in Fig. 5.15 (a). As shown in the figure, the width of the coplanar waveguide at both ends of the SWS is tapered to attain a wideband matching. Fig. 5.15(b) shows the bottom horizontal strips of the PH-SEC, which are printed on the lower substrate. Fig. 5.15(c) shows the straight-edge connections of the PH-SEC, realized using copper wires. Figs. 5.15(a)-(b) show the circular patches of $0.7 \mathrm{~mm}$ diameter at the ends of the horizontal strips on top and bottom dielectric substrates. The assembled SWS without the top metal cover is shown in Fig. 5.15(d).

\subsubsection{Measured Results}

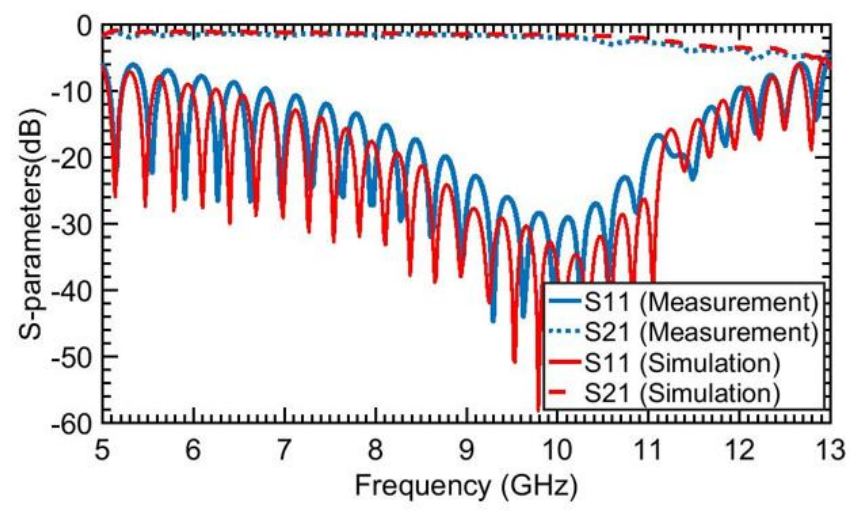

Figure 5.16: Comparison of measured and simulated S-parameters.

The S-parameters and phase velocity of the fabricated SWS are measured and compared with those from simulation. Transient solver in CST microwave studio is used for the simulations of the complete SWSs with different number of periods. Perfect electric boundaries are placed at suitable separation around the device. The conductor loss is included in the simulations by considering copper with conductivity of $2.9 \times 10^{7} \mathrm{~S} / \mathrm{m}$. The dielectric loss is included by considering loss tangent of 0.0027 for the substrates. 
Comparison between simulated and measured S-parameter values of the SWS with thirty periods is shown in Fig. 5.16. The measured values of S21 show good match with those from simulations over $7 \mathrm{GHz}$ to $12 \mathrm{GHz}$, which is the desired frequency range. Moreover, the S11 values from simulations also match very well the measured values over this frequency range.

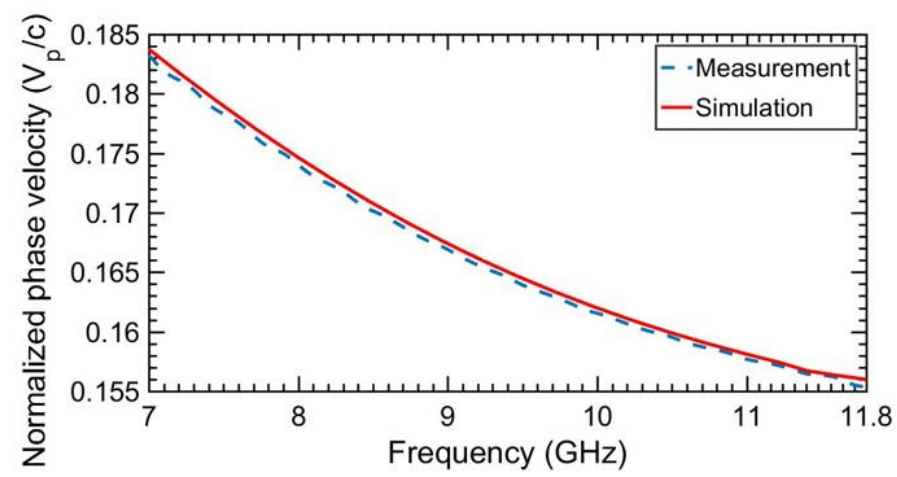

Figure 5.17: Measured and simulated normalized phase velocity of the SWS.

The phase constant of the PH-SEC is calculated from the measured phase values of S21 of the SWSs with two different lengths. The phase velocity is measured as explained in Section 4.3.2. The comparison between the phase velocity from the measurement and simulation is shown in Fig. 5.17. As presented, the measured phase velocity values have a close match with those from simulation over the frequency range $7 \mathrm{GHz}$ to $11.8 \mathrm{GHz}$. The phase velocity values from the measurement are slightly lower than those from simulation. This difference may be due to the fabrication tolerances, extra length of the straight-edge connections at solder joints, and the slight difference in the conductivity values for the fabricated structure and those used in simulation.

\subsection{Summary}

In this chapter, the design and performance of a W-band BWO based on the microfabrication-compatible PH-SEC are presented. The design of the SWS with its dispersion characteristics and interaction impedance is presented. The results from particlein-cell (PIC) simulations of the BWO show that for a beam voltage varying from $7 \mathrm{KV}$ to $11 \mathrm{KV}$ the device can generate signals with frequency varying from $86.9 \mathrm{GHz}$ to $100 \mathrm{GHz}$, corresponding to a tuneable bandwidth of $14 \%$. The performance of the oscillator with three different beam currents ( $20 \mathrm{~mA}, 18 \mathrm{~mA}$, and $16 \mathrm{~mA})$ is studied. The oscillator can generate a peak output power of $2.3 \mathrm{~W}$ and it can give a maximum DC-to-RF efficiency of $1.62 \%$. 
To validate the simulation results, a scaled version of the PH-SEC SWS designed to operate at X-band frequencies is fabricated. The measured S-parameters and the phase velocity of the SWS closely match those from simulations.

The presented BWO has a very good potential of serving as a signal source at Wband frequencies. This concept of the BWO is expected to be able to work at frequencies higher than W-band also since the SWS is microfabrication-compatible. Moreover, this oscillator is expected to be able to be fabricated together with the PH-SEC based amplifier to realize a system which can generate high frequency high power signals. 


\section{Chapter 6 Two-Beam Oscillator-Amplifier}

\subsection{Introduction}

Even though the backward-wave oscillators (BWOs) have unique features like electronic frequency tuneability and wide tuneable bandwidth, in many cases their low DCto-RF conversion efficiency constitutes a drawback. This problem is severe for the BWOs with low beam voltage, i.e., nonrelativistic BWOs. The relativistic BWOs have higher efficiency and output power compared to that of the nonrelativistic BWOs since they have stronger interaction between the electron beam and the electromagnetic wave. But realizing and maintaining a high power electron beam for the relativistic BWOs is challenging, Here, we focus attention on the nonrelativistic BWOs. The efficiency of these devices drops substantially with increase in the frequency of operation. For instance, a nonrelativistic BWO with folded waveguide SWS has been designed to operate at $35 \mathrm{GHz}$; the oscillator gives a peak efficiency of $1.74 \%$ and an output power of $20 \mathrm{~W}$ [106]. Another folded waveguide SWS based BWO with $12 \mathrm{KV}$ beam voltage has been designed to operate around $95 \mathrm{GHZ}$, giving an electronic efficiency of $0.84 \%$ and $5 \mathrm{~W}$ output power [25]. Only watt level of output power at $90 \mathrm{GHz}$ has been estimated from a BWO with staggered double-vane SWS employing an electron beam with input DC power of $2 \mathrm{KW}$ [71].

Some modified devices such as orotron and clinotron have been proposed to overcome the low output power and efficiency of conventional BWOs [2]. The orotron has a Fabry-Perot cavity made up of two mirrors, in which one mirror is associated with a periodic structure. Larger interaction volume of the open resonator in orotrons generates higher output power than BWOs. As compared to a conventional BWO at W-band which generates $0.7 \mathrm{~W}$ of output power, the orotron generates $1 \mathrm{~W}$ output power at the same frequency range by using the same electron beam [107]. However, the orotron cavity has a higher ohmic loss due to the higher amplitude of the electromagnetic fields in the high-Q cavity compare to that of the output wave. Besides, the ohmic loss is again increased due to the low group velocity of the quasi-cutoff operating wave in the cavity. Increased ohmic loss in orotron impedes its application in the continuous wave $(\mathrm{CW})$ regime. In comparison, the BWOs do not have this issue since the travelling-wave in them does not allow build-up of power in the interaction region [107], [108]. 
The clinotrons use an inclined electron beam. The inclined electron beam has a stronger interaction with the evanescent surface wave of the slow-wave structure (SWS). At the same time, the inclined electron beam reduces the device performance due to noise generation and heating of the SWS. Besides, the efficiency of this device cannot be improved using a depressed collector [109]. A fair comparison between the electronic efficiency of the clinotron and BWO using similar interaction structure and electron beam shows that the efficiency of the clinotron is $0.068 \%$ while that of the BWO is $0.021 \%$ [110].

Different techniques have been proposed in the past for improving the efficiency of nonrelativistic BWOs. Generally, travelling-wave tube amplifiers (TWTAs) are used for amplifying the output signal from the BWO to generate high power electromagnetic signals [111]. But this simple technique increases the size and cost of the signal generator. Prebunched electron beam has been proposed for improving the efficiency of a conventional BWO [112]. Theoretical studies estimated that the pre-bunched beam can improve the efficiency by a factor of 2.4 (from 15\% to $35 \%$ ). But studies on the tuneability of this technique have not been reported yet. Practical realization of this technique would need a complex design. Tandem BWO-TWT system is a more practical method for the efficiency improvement of BWOs. This technique uses an additional section of slow-wave structure (SWS) with a conventional BWO. The electron beam in the BWO should be velocity synchronized with the forward-wave of the additional section of the SWS [113]-[114]. Although this technique has been demonstrated for relativistic BWOs, the same technique should be applicable to nonrelativistic BWOs as well. An experiment on relativistic tandem BWO-TWT system has shown that the system can provide an efficiency improvement of 2.4 times [113]. But the tuneable bandwidth of the BWO reduced from $\sim 18 \%$ to $\sim 9 \%$ with this technique. Increase in the overall device length and reduction of the tuneable bandwidth are the drawbacks of this technique.

SWS with deep corrugations and inhomogeneous pitch has been used for the design of a Ka-band BWO with relatively high beam voltage. The BWO with these modifications can improve the efficiency from $20 \%$ to $58 \%$ with a maximum output power of $5.92 \mathrm{MW}$ [70]. But this design also sacrifices the tuneable bandwidth of the oscillator. Thus, a technique to improve the efficiency of BWOs that also achieves wideband tunability as well as compact size seems lacking. 
In this context, here we present a new technique to improve the efficiency of the conventional BWO by including an additional electron beam. The proposed technique does not compromise the tuneable bandwidth and the overall size of the BWO. The efficiency improvement of the proposed technique is verified using the results from simulation. Computer simulation technology (CST) microwave studio is used for the simulations. Although the challenge in the proposed technique is the requirement of two electron beams with different beam voltages in a single device, two electron beams with different beam voltages have been realised in the past for application in electron wave tubes [115]-[116]

The concept of the proposed two-beam oscillator-amplifier is explained in Section 6.2 of this chapter. A conventional BWO and the proposed oscillator-amplifier are designed using a Ku-band circular helix SWS, and the performance of both devices is compared for three different beam configurations. Section 6.3 presents the design and beam configurations for both devices. The output performance of both oscillator-amplifier and BWO is obtained using the particle-in-cell (PIC) simulator and is compared in Section 6.4. Next, the planar helix slow-wave structure with straight-edge connections (PH-SEC) is also used for designing both BWO and oscillator-amplifier at Ku-band. The design of both devices is described in Section 6.5 and Section 6.6 compares the results from PIC simulation of both devices. A summary of this chapter is presented in Section 6.7.

\subsection{Concept of the Proposed Technique}

The block diagram of a travelling-wave tube (TWT) is given in Fig. 6.1 (a). As mentioned in Chapter 1, the output signal for a conventional BWO is generated at port-1 and port- 2 is match-terminated. The electron beam for the conventional BWO is velocity synchronized with the $n=-1$ space harmonic of the SWS. On the other hand, for operating the TWT as a TWTA, one feeds the input signal at port-1 and the output signal is obtained from port-2. The electron beam for the TWTA is velocity synchronized with the fundamental space harmonic of the SWS.

Fig. 6.1 (b) show the block diagram of the proposed oscillator-amplifier. The structure of the proposed oscillator-amplifier has two changes compared to that of a conventional BWO. First change is the inclusion of a second electron beam in the BWO. The second electron beam is velocity synchronized with the phase velocity of the fundamental space harmonic of the SWS. As the second change, port-1 of the SWS is terminated into an open circuit (or a short circuit). 


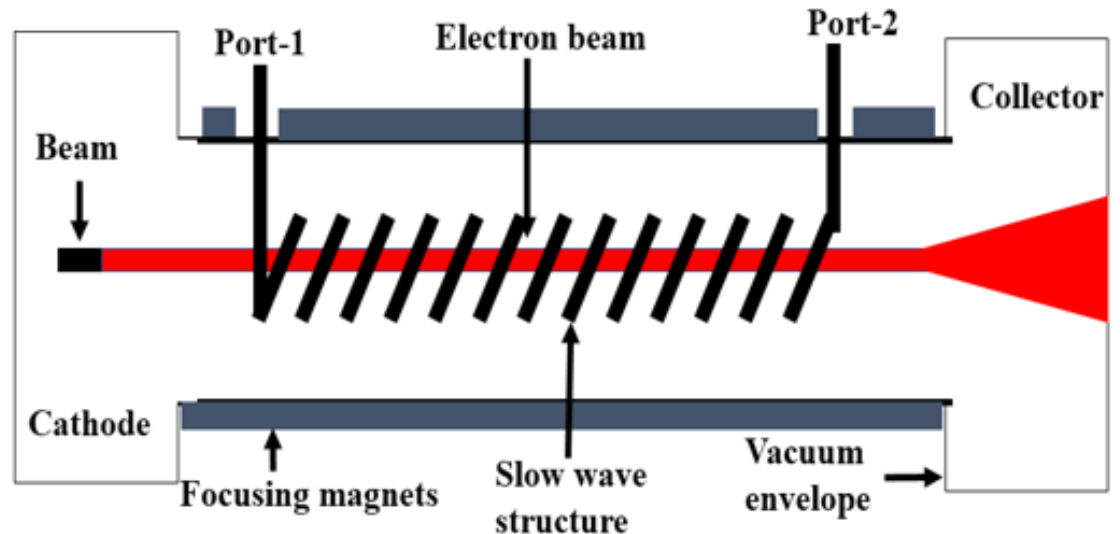

(a)

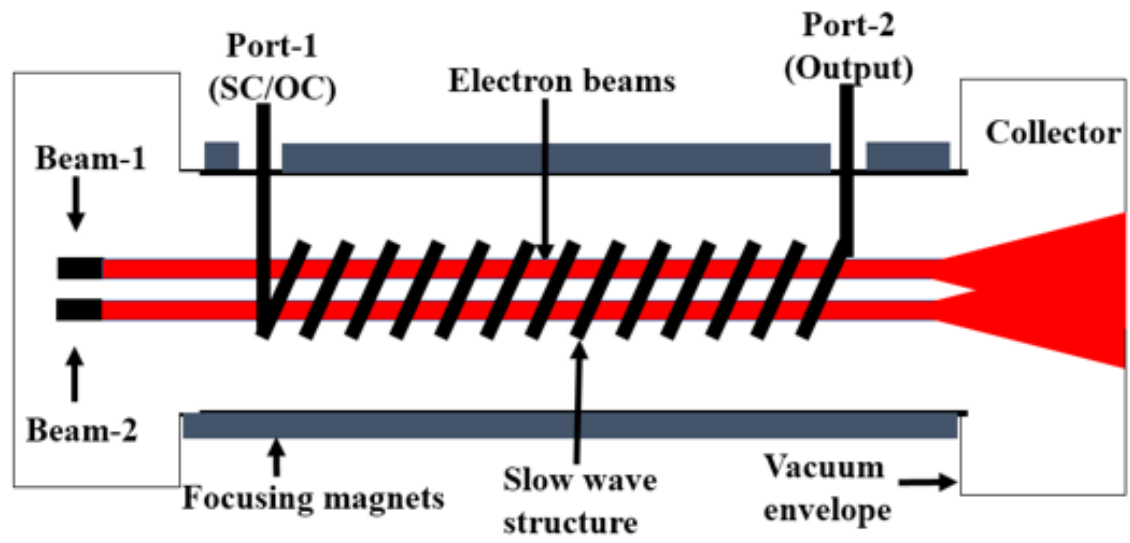

(b)

Figure 6.1: Schematic diagram of the (a) traveling-wave tube (TWT); (b) proposed vacuum electronic two-beam oscillator-amplifier.

The operation of the oscillator-amplifier can be explained by combining the operating principles of both the BWO and TWTA. Beam-1 in Fig. 6.1(b) is velocity synchronized with the $n=-1$ space harmonic of the SWS, generating an RF signal at port1 according to the usual BWO operation. This signal is fully reflected back to port-2 through the SWS since the port-1 is open circuited (or short circuited). This reflected signal acts as an input signal applied at port- 1 of a TWTA, interacts with beam-2 which is velocity synchronized with the fundamenral space harmonic of the SWS, gets amplifed and the final output is obtained from port-2. The proposed oscillator-amplifier combines the operation of both BWO and TWTA in a single SWS without increasing the overall length. The efficiency of the two-beam oscillator-amplifier is a combination of the efficiency of both the BWO and TWTA. 


\subsection{Design of BWO and Oscillator-Amplifier Using Circular Helix}

The popular circular helix SWS is used first for the design of both BWO and oscillator-amplifier. The structure, dimensions, dispersion characteristics, and interaction impedance of the circular helix SWS are presented in the following sub-sections. The following sub-sections also present the transmission and reflection characteristics of the SWS for both BWO and oscillator-amplifier. The section ends with a description of three different beam configurations for the two devices.

\subsubsection{Properties of the SWS}
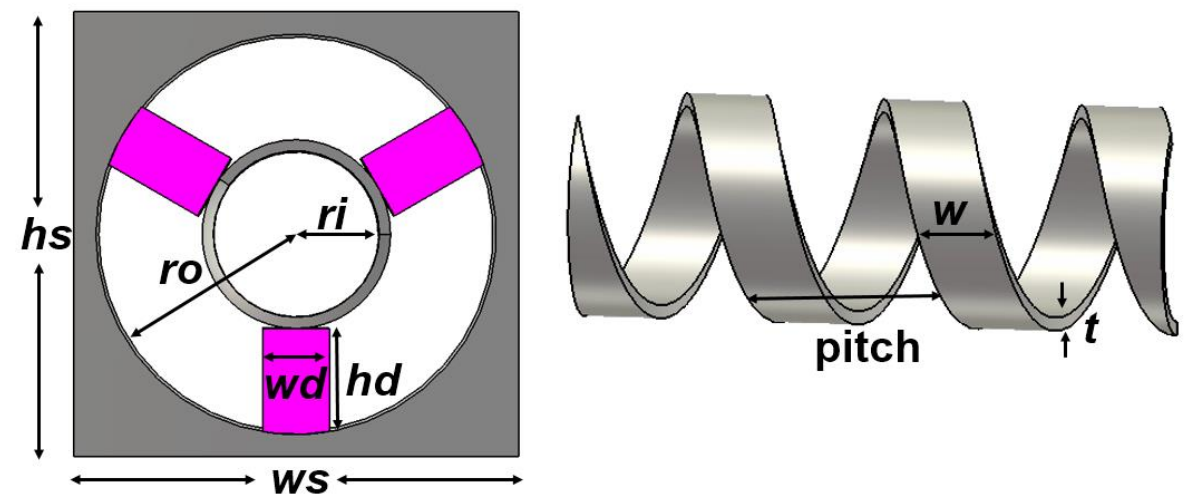

Figure 6.2: Cross-section and perspective views of the circular helix SWS used for designing BWO and oscillator-amplifier.

Table 6.1 Dimensions of the SWS

\begin{tabular}{|r|r|r|r|}
\hline Parameter & Value (mm) & Parameter & Value (mm) \\
\hline$r i$ & 0.75 & $h s$ & 4 \\
\hline$r o$ & 1.8 & pitch & 1.5 \\
\hline$w d$ & 0.6 & $w$ & 0.6 \\
\hline$h d$ & 0.95 & $t$ & 0.1 \\
\hline$w s$ & 4 & & \\
\hline
\end{tabular}

Circular helix SWS as shown in Fig. 6.2 is used for the design of both devices. As shown in the figure, the circular helix is supported inside a metal enclosure using three anisotropic pyrolytic boron nitride (APBN) dielectric slabs. The APBN dielectric is considered to have a dielectric constant of 5.1 and loss tangent of 0.005 . Dimensions of the SWS to operate at Ku-band are given in Table 6.1.

The dispersion characteristics of the SWS are evaluated using the CST eigenmode solver. Phase constant versus frequency for two modes of the SWS, and the beam lines 
corresponding to three different beam voltages are shown in Fig. 6.3. Mode-1 is the fundamental mode of the SWS whereas mode-2 is the first higher order mode. As shown in the figure, the beam lines corresponding to $4 \mathrm{KV}$ and $6 \mathrm{KV}$ beam voltages intersect with the $n=-1$ space harmonic of the fundamental mode at $16.7 \mathrm{GHz}$ and $18.9 \mathrm{GHz}$, respectively. These intersections indicate that the electron beam with $4 \mathrm{KV}$ and $6 \mathrm{KV}$ beam voltages can generate signals at $16.7 \mathrm{GHz}$ and $18.9 \mathrm{GHz}$, respectively. The beam line corresponding to 17.2 KV beam voltage almost coincides with the fundamental space harmonic of mode-1 for a wide range of frequency including $16.7 \mathrm{GHz}$ and $18.9 \mathrm{GHz}$. This indicates that the 17.2 KV beam voltage can amplify a wide range of frequency.

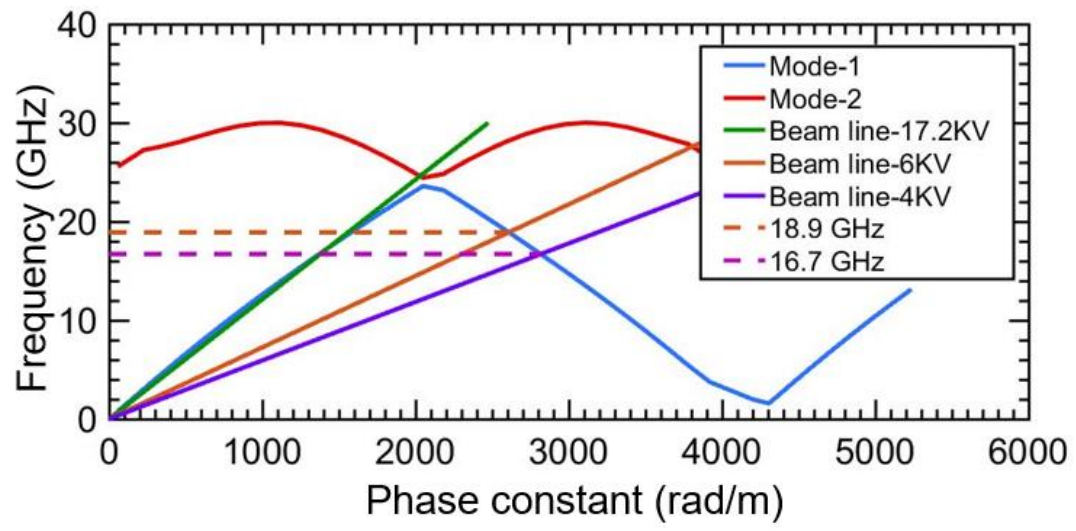

Figure 6.3: Dispersion characteristics of the SWS with three beam lines.

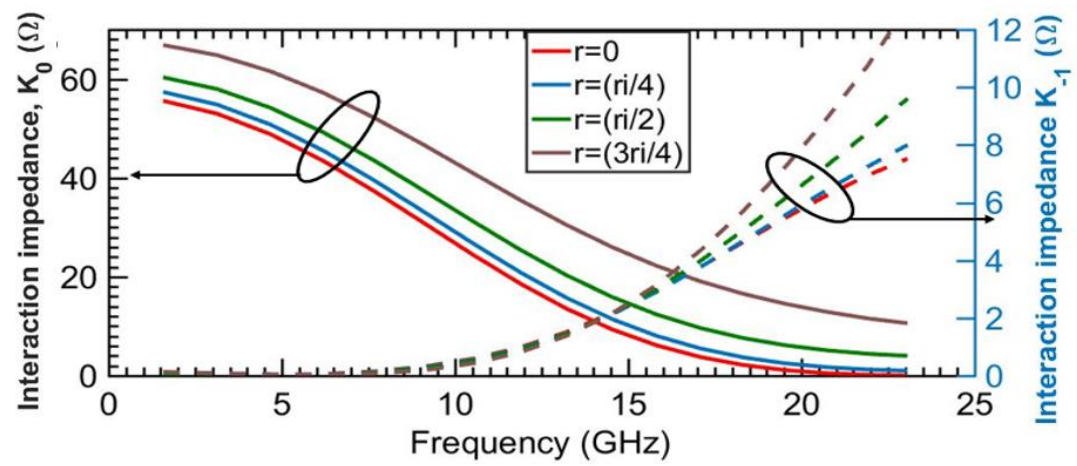

Figure 6.4: Interaction impedance of the fundamental $\left(\mathrm{K}_{0}\right)$ and $\mathrm{n}=-1\left(\mathrm{~K}_{-1}\right)$ space harmonic of the circular helix SWS at four different radial positions.

The interaction impedance versus frequency for both the fundamental $\left(\mathrm{K}_{0}\right)$ and the $\mathrm{n}=-1\left(\mathrm{~K}_{-1}\right)$ space harmonic of mode-1 of the SWS at four different radial positions is shown in Fig. 6.4. As expected, the fundamental space harmonic has a higher interaction impedance than that of the $n=-1$ space harmonic. The value of $K_{0}$ decreases with frequency 
whereas that of $\mathrm{K}_{-1}$ increases with frequency for a given radial position. The interaction impedance of both space harmonics has a higher value at a radial position closer to the helix.

\subsubsection{Transmission and Reflection Characteristics}

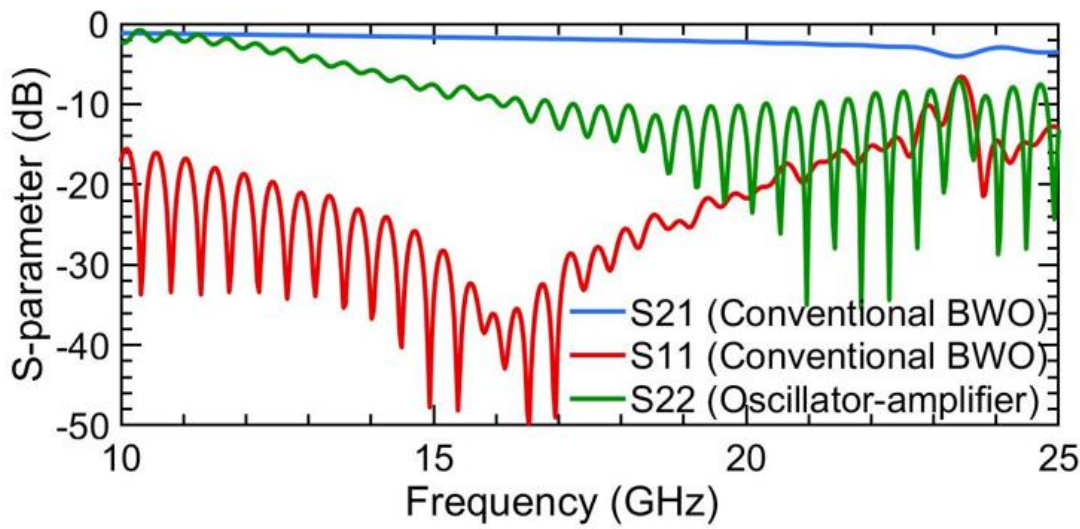

Figure 6.5: S-parameters of the SWS used as BWO and as oscillator-amplifier.

Fifty periods of the circular helix shown in Fig. 6.2 are combined to form the SWS for both BWO and oscillator-amplifier. The interaction length and structure of the SWS for both devices are kept the same for a fair comparison. Both port-1 and port-2 of the SWS are considered to be matched for the simulation of the BWO. The port- 1 is considered to be a perfect open-circuit and port-2 is considered to be matched for the simulation of the oscillator-amplifier. The reference impedance for port-2 is $93 \Omega$. One can change it to 50 $\Omega$ by pitch tapering (i.e., impedance matching), but it will not affect the final result.

The S-parameters of both devices are obtained from the CST transient solver and are shown in Fig. 6.5. The conventional BWO has S11 less than $-20 \mathrm{~dB}$ and S21 better than $1.2 \mathrm{~dB}$ for the $13-20 \mathrm{GHz}$ frequency range. This frequency range represents a bandwidth of $42 \%$; this can also be considered to be the expected bandwidth of operation of the conventional BWO. The proposed oscillator-amplifier is a one port device and it has an S22 only better than $-9 \mathrm{~dB}$ for frequencies from 13-20 GHz. The complete mismatch at port-1 is the reason for the poor reflection characteristics of the oscillator-amplifier.

\subsubsection{Beam Configurations}

For studying the performance of the oscillator-amplifier, three different beam configurations are considered. As shown in Fig. 6.6, the $1^{\text {st }}$ beam configuration uses a solid cylindrical beam for forward-wave interaction and an annular beam for backward-wave interaction. Annular beam is used to improve the interaction between electron beam and $n=$ 
-1 space harmonic [117]-[118]. Both beams are coaxial with the circular helix. The annular beam has an inner radius of $0.4 \mathrm{~mm}$ and an outer radius of $0.55 \mathrm{~mm}$, while the solid beam has a radius of $0.25 \mathrm{~mm}$. The $2^{\text {nd }}$ configuration uses two solid cylindrical beams each with a radius of $0.25 \mathrm{~mm}$ and a separation of $0.2 \mathrm{~mm}$ between them. The $3^{\text {rd }}$ configuration also uses two solid cylindrical beams each with a radius of $0.25 \mathrm{~mm}$. The beam for the backward-wave interaction is placed outside the helix while the beam for the forward-wave interaction is placed inside and is coaxial with the helix. The beam for the backward-wave interaction in the $3^{\text {rd }}$ beam configuration has a separation of $1 \mathrm{~mm}$ from the circular helix. The two different beams in all beam configurations are considered to be generated from two different electron guns that can have different cathode temperatures, beam voltages and beam currents.

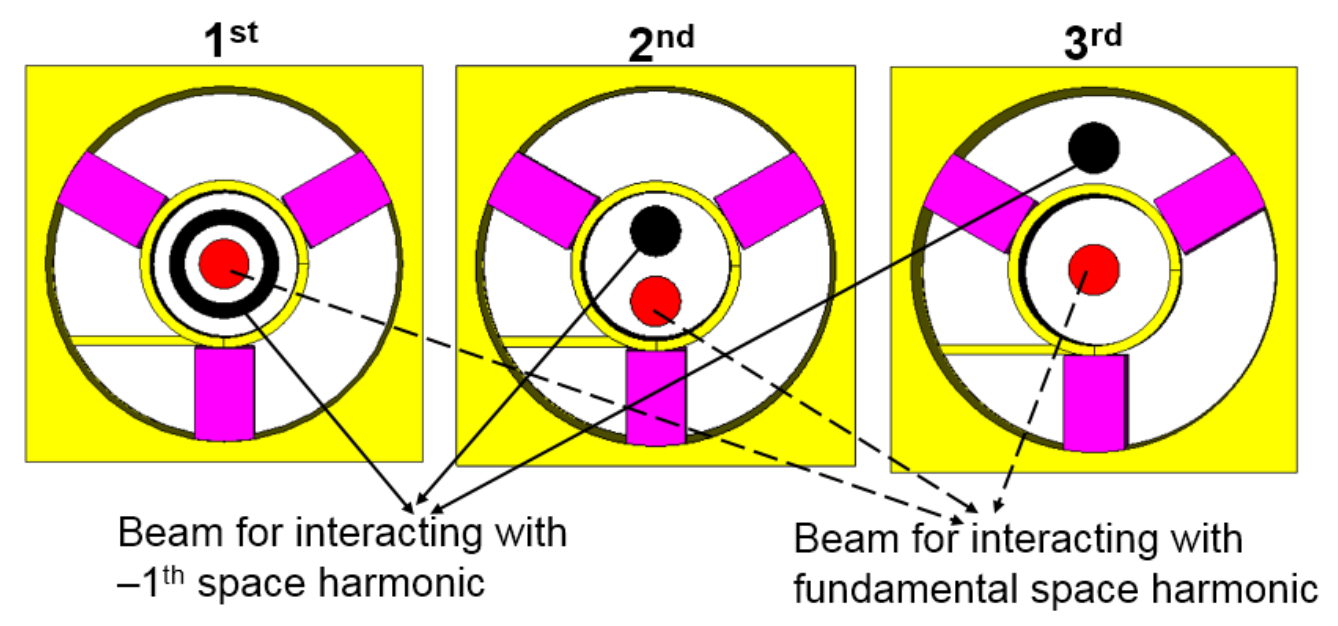

Figure 6.6: Three configurations of the beams considered for the proposed oscillatoramplifier.

Only the beam intended for the backward-wave interaction is considered for the simulations of the conventional BWO. For a fair comparison, the shape, position, and size of the beam corresponding to the backward-wave interaction for both conventional BWO and oscillator-amplifier are kept the same for all three beam configurations.

\subsection{Comparison of PIC Simulation Results}

The output characteristics of both BWO and oscillator-amplifier are simulated using the PIC solver and are compared for the three different beam configurations in the following sub-sections. The full structure of the SWS and the electron beam are simulated in the PIC solver. Perfect electric boundary is applied at all boundaries of the device during the simulation. 
Table 6.2 Comparison of output performance of the conventional BWO and the twobeam oscillator-amplifier

\begin{tabular}{|l|l|l|l|l|l|l|l|l|l|l|}
\hline $\begin{array}{c}\text { Beam } \\
\text { config } \\
\text { uration }\end{array}$ & $\begin{array}{c}\text { Beam parameters for } \\
\text { backward-wave } \\
\text { interaction }\end{array}$ & $\begin{array}{c}\text { Beam parameters for } \\
\text { forward-wave } \\
\text { interaction }\end{array}$ & \multicolumn{2}{|c|}{$\begin{array}{c}\text { Output signal from the conventional } \\
\text { BWO (with one beam) }\end{array}$} & \multicolumn{3}{|c|}{$\begin{array}{c}\text { Output signal from the oscillator- } \\
\text { amplifier (with two beams) }\end{array}$} \\
\cline { 2 - 13 } & $\begin{array}{c}\text { Voltage } \\
(\mathrm{KV})\end{array}$ & $\begin{array}{c}\text { Current } \\
(\mathrm{A})\end{array}$ & $\begin{array}{c}\text { Voltage } \\
(\mathrm{KV})\end{array}$ & $\begin{array}{c}\text { Current } \\
(\mathrm{A})\end{array}$ & $\begin{array}{c}\text { Power } \\
(\mathrm{W})\end{array}$ & $\begin{array}{c}\text { Efficiency } \\
(\%)\end{array}$ & $\begin{array}{c}\text { Frequency } \\
(\mathrm{GHz})\end{array}$ & $\begin{array}{c}\text { Power } \\
(\mathrm{W})\end{array}$ & $\begin{array}{c}\text { Efficiency } \\
(\%)\end{array}$ & $\begin{array}{c}\text { Frequency } \\
(\mathrm{GHz})\end{array}$ \\
\hline $1^{\text {st }}$ & 6 & 0.05 & 17.2 & 0.15 & 21.2 & 7.05 & 18.4 & 420 & 14.6 & 18.4 \\
\hline $1^{\text {st }}$ & 4 & 0.05 & 17.2 & 0.15 & 12.3 & 6.13 & 16.2 & 324 & 11.7 & 16.3 \\
\hline $2^{\text {nd }}$ & 6 & 0.25 & 17.2 & 0.15 & 67.2 & 4.48 & 18.1 & 625 & 15.3 & 17.6 \\
\hline $2^{\text {nd }}$ & 4 & 0.25 & 17.2 & 0.15 & 43.2 & 4.32 & 15.8 & 600 & 16.8 & 15.8 \\
\hline $3^{\text {rd }}$ & 6 & 0.4 & 17.2 & 0.15 & 43.5 & 1.82 & 17.3 & 615 & 12.1 & 17.2 \\
\hline $3^{\text {rd }}$ & 4 & 0.4 & 17.2 & 0.15 & 24.4 & 1.53 & 14.2 & 434 & 10.4 & 14.1 \\
\hline
\end{tabular}

\subsection{1 $1^{\text {st }}$ Beam Configuration}

The $1^{\text {st }}$ beam configuration uses an annular beam closer to the helix for backwardwave interaction. Stronger interaction between the electron beam and the $n=-1$ space harmonic of the SWS is obtained with the annular beam since the interaction impedance for the $n=-1$ space harmonic is stronger at a location closer to the helix. The beam current for the conventional BWO is $0.05 \mathrm{~A}$ and a magnetic field of $0.25 \mathrm{~T}$ is used for focusing the electron beam. The annular beam with a beam current of $0.05 \mathrm{~A}$ and the solid beam with a beam current of $0.15 \mathrm{~A}$ are used for the corresponding simulations of the oscillator-amplifier. The simulations of the oscillator-amplifier use an increased focusing magnetic field of 0.4 $\mathrm{T}$ for focusing both beams.

The simulated time-domain output signal for the conventional BWO with the annular beam of $4 \mathrm{KV}$ beam voltage is shown in Fig. 6.7. The figure also includes the simulated time-domain output signal from the oscillator-amplifier with the $4 \mathrm{KV}$ annular beam and the 17.2 KV solid beam. The output signal from both devices grows with time and settles to a steady value. The amplitude of the output signal from the oscillator-amplifier is almost five times higher than that from the conventional BWO. The tuneability of the oscillatoramplifier is studied by simulating the device at two different beam voltages for the annular beam while keeping the beam voltage for the solid beam as constant. As shown in Fig. 6.8, the frequency of the output signal reduces from $18.4 \mathrm{GHz}$ to $16.3 \mathrm{GHz}$ as the beam voltage for the beam corresponding to backward-wave interaction reduces from $6 \mathrm{KV}$ to $4 \mathrm{KV}$. 


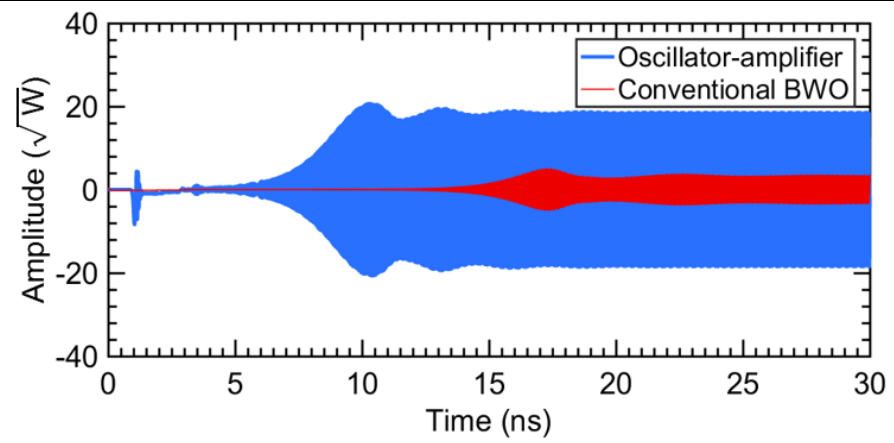

Figure 6.7: Output signal from both oscillator-amplifier and conventional BWO. the oscillator-amplifier uses a $4 \mathrm{KV}$ annular beam and a $17.2 \mathrm{KV}$ solid beam, whereas the conventional BWO uses the $4 \mathrm{KV}$ annular beam ( $1^{\text {st }}$ beam configuration).

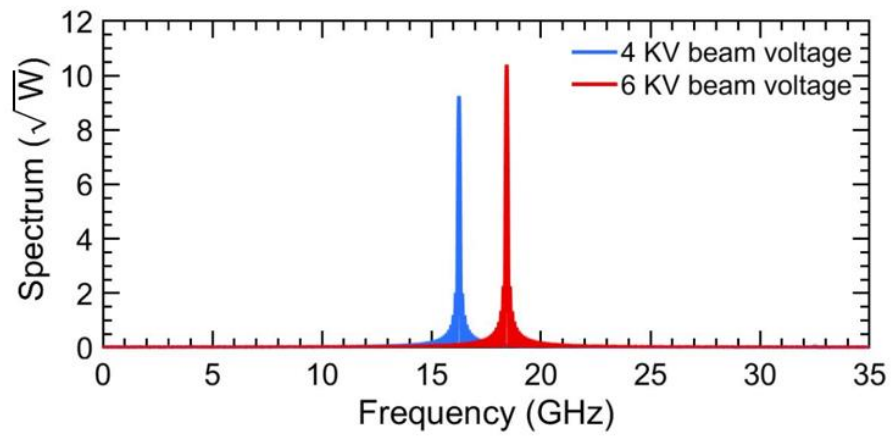

Figure 6.8: Discrete Fourier transform of the output signal from the oscillator-amplifier at two different beam voltages for the annular beam ( $1^{\text {st }}$ beam configuration).

The DC-to-RF conversion efficiency of the conventional BWO is calculated as the ratio between the output RF power and DC power of the electron beam and is given as:

$$
\eta=\frac{\text { Power of output RF signal }}{\text { Input power (E-beam for backward-wave interaction })} x 100
$$

whereas the DC-to-RF conversion efficiency of the oscillator-amplifier is calculated as the ratio between output RF power and the DC power contributed by both electron beams and is given as:

$$
\eta=\frac{\text { Power of output RF signal }}{\left(\text { Input power of } 1^{\text {st }} \text { E-beam+Input power of } 2^{\text {nd }} \text { E-beam }\right)} \times 100
$$

The amplitude of the output signal from the PIC simulations is square root of the RF output power (Fig. 6.7 and other similar figures). Therefore, the RF output power is calculated by taking the square of the amplitude of the output signal. The DC power of each 
of the electron beams is calculated as the product of their respective beam voltage and beam current.

The simulation results for both the conventional BWO and the oscillator-amplifier with the $1^{\text {st }}$ beam configuration at two different beam voltages for the annular beam are given in Table 6.2. The table shows that the DC-to-RF conversion efficiency of the oscillator-amplifier for this beam configuration is close to two times that of the conventional BWO. Besides, the output power of the oscillator-amplifier is twenty times or more compared to that of the conventional BWO.

\subsection{2 $2^{\text {nd }}$ Beam Configuration}

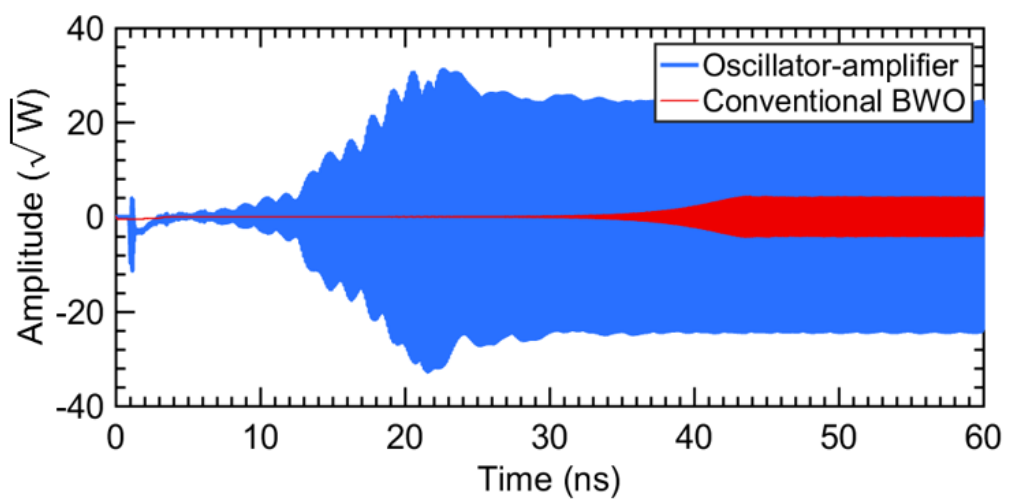

Figure 6.9: Output signal from the oscillator-amplifier with $4 \mathrm{KV}$ and $17.2 \mathrm{KV}$ solid beams, and the corresponding output signal from the conventional BWO with $4 \mathrm{KV}$ solid beam ( $2^{\text {nd }}$ beam configuration).

Practical realization of the $1^{\text {st }}$ beam configuration is challenging since it has an annular beam coaxial with a solid beam. As an alternative, we consider the $2^{\text {nd }}$ beam configuration with two solid beams as shown in Fig. 6.6. In this configuration, a solid beam with beam current of $0.25 \mathrm{~A}$ is used for the conventional BWO. Corresponding simulations of the oscillator-amplifier use an additional solid beam with beam current of $0.15 \mathrm{~A}$ for the forward-wave amplification. Simulations of both devices use a focusing magnetic field of $0.5 \mathrm{~T}$.

The output signal versus time for the oscillator-amplifier with a $4 \mathrm{KV}$ solid beam for backward-wave interaction and with a $17.2 \mathrm{KV}$ solid beam for forward-wave interaction is given in Fig. 6.9. The time-domain output signal from the corresponding conventional BWO with a $4 \mathrm{KV}$ solid beam for backward-wave interaction is also included in this figure. Here also the output signal grows with time and settles to a steady value for both devices. 


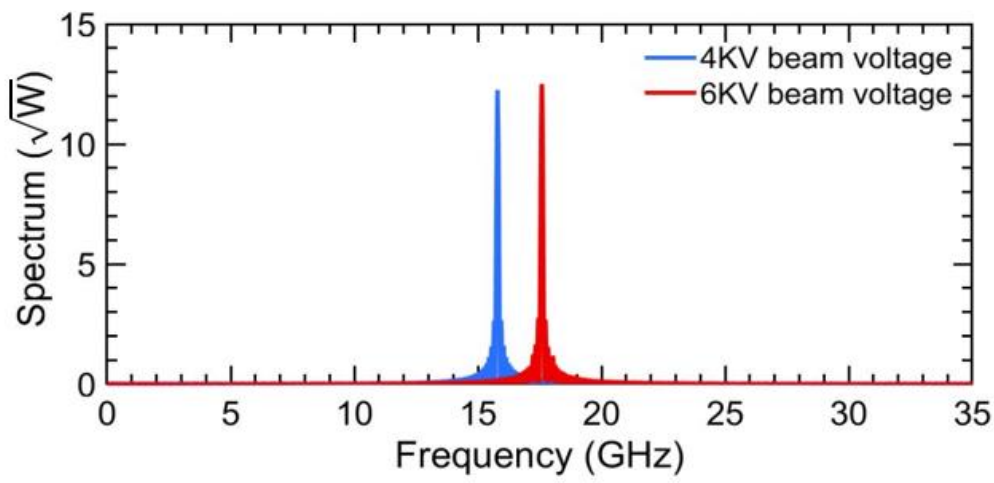

Figure 6.10: Discrete Fourier transform of the output signal from the oscillator-amplifier at two different beam voltages ( $2^{\text {nd }}$ beam configuration).

The tuneability of the oscillator-amplifier is studied by simulating the device with different beam voltages for the solid beam corresponding to the backward-wave interaction. As shown in Fig. 6.10, the frequency of the output signal is $15.8 \mathrm{GHz}$ for a beam voltage of $4 \mathrm{KV}$ and it changes to $17.6 \mathrm{GHz}$ for the beam voltage of $6 \mathrm{KV}$.
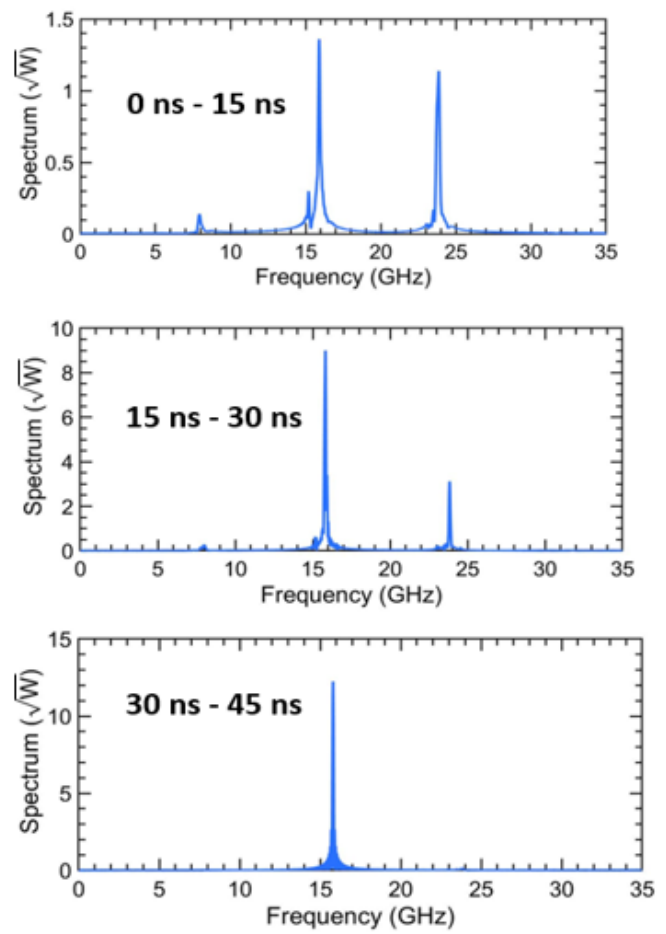

Figure 6.11: Discrete Fourier transform of the output signal from the oscillator-amplifier with $4 \mathrm{KV}$ and $17.2 \mathrm{KV}$ solid beams for three different time intervals.

The output signal from the oscillator-amplifier presented in Fig. 6.9 shows some instability at the start of oscillations. This instability is studied by analysing the discrete Fourier transform (DFT) of the output signal at three different time intervals. As shown in 
Fig. 6.11, the output signal has frequency components at $15.8 \mathrm{GHz}$ and $23.9 \mathrm{GHz}$ for first $15 \mathrm{~ns}$. The frequency component of $23.9 \mathrm{GHz}$ is generated due to the unintentional intersection of the $17.2 \mathrm{KV}$ beam line with the $\mathrm{n}=-1$ space harmonic of mode- 2 of the SWS. But the amplitude of this frequency component gradually diminishes to a very low value with time. The frequency of the output signal settles to the desired value of $15.8 \mathrm{GHz}$ at around 30 ns.

The simulation results for both the conventional BWO and the oscillator-amplifier with the $2^{\text {nd }}$ beam configuration at two different beam voltages for the backward-wave interaction are also tabulated in Table 6.2. For this beam configuration, the DC-to-RF conversion efficiency of the oscillator-amplifier is improved compare to that of the conventional BWO by a factor of three or more. Furthermore, the output power of the oscillator-amplifier is nine times or more compared to that of the conventional BWO.

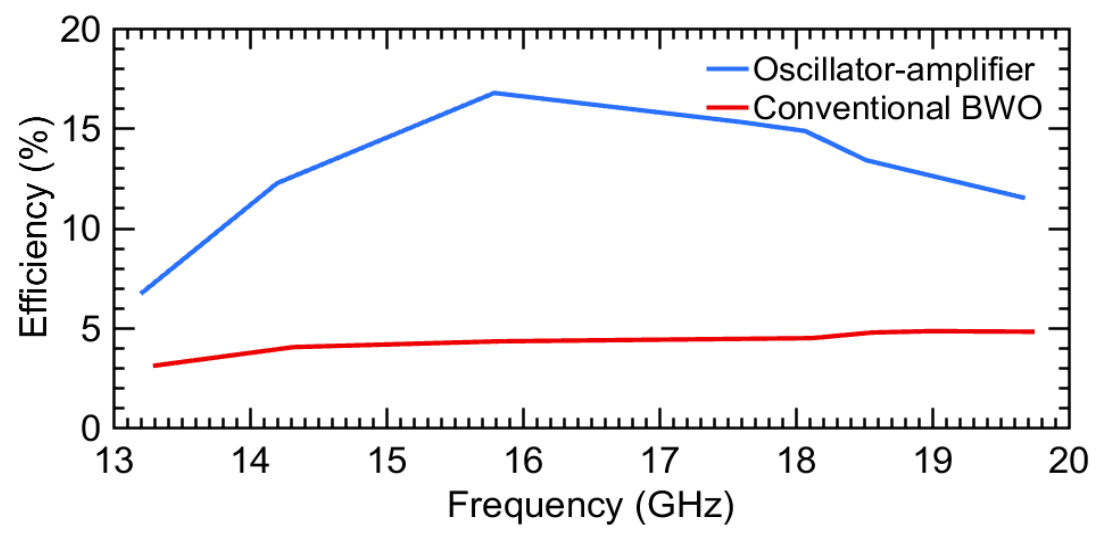

Figure 6.12: Comparison of efficiency of the oscillator-amplifier and the conventional BWO.

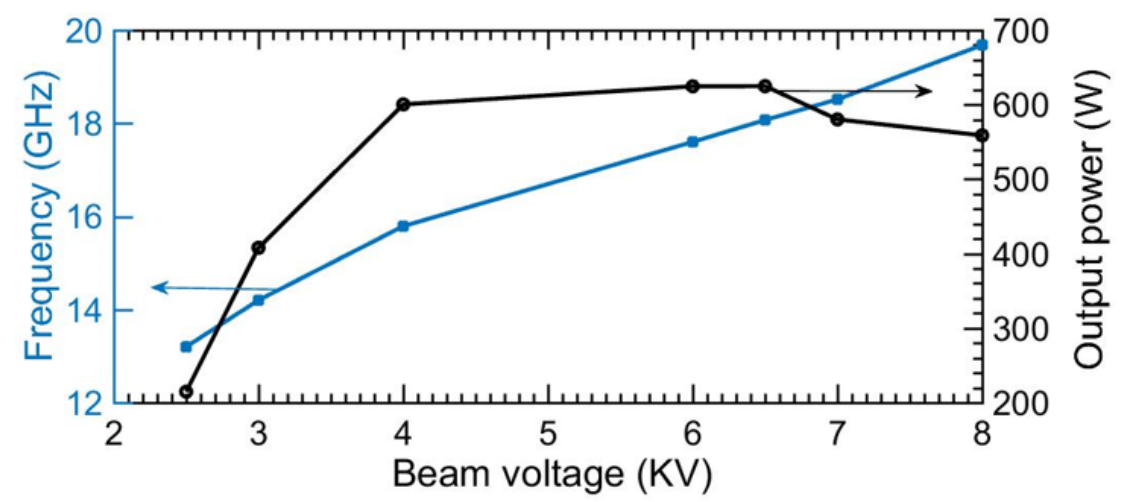

Figure 6.13:Variation of frequency and power of the output signal with beam voltage for backward-wave interaction in the oscillator-amplifier. 
The tuneable bandwidth of both the conventional BWO and the oscillator-amplifier with the $2^{\text {nd }}$ beam configuration is examined by varying the beam voltage for the backwardwave interaction from $2.5 \mathrm{KV}$ to $8 \mathrm{KV}$ while keeping the beam voltage for the forwardwave interaction constant. The frequency of the output signal from both devices varies from 13.2 $\mathrm{GHz}$ to $19.7 \mathrm{GHz}$ as the beam voltage varies from $2.5 \mathrm{KV}$ to $8 \mathrm{KV}$. This corresponds to a tuning bandwidth of $39 \%$, showing clearly that the oscillator-amplifier does not compromise the tuneable bandwidth of the conventional BWO. The efficiency of both devices over this frequency range is given in Fig. 6.12. As shown in the figure, the oscillator-amplifier has an efficiency improvement of more than 2 times compared to that of the conventional BWO over the entire tuneable bandwidth. Maximum efficiency improvement is obtained at a frequency near $16 \mathrm{GHz}$ since the $17.2 \mathrm{KV}$ beam voltage can achieve best synchronization with the fundamental space harmonic at this frequency. The power and frequency of the output signal from the oscillator-amplifier versus beam voltage for backward-wave interaction are shown in Fig. 6.13. The figure shows that the oscillatoramplifier can generate a maximum output power of $625 \mathrm{~W}$.

\subsection{3 $3^{\text {rd }}$ Beam Configuration}

The $3^{\text {rd }}$ beam configuration shown in Fig. 6.6 is considered for increasing the separation between the two beams so as to reduce the complexity in achieving them. In this configuration the beam for the backward-wave interaction is kept outside the SWS and the beam for the forward-wave interaction is kept inside the SWS. The beam current of the conventional BWO increases to $0.4 \mathrm{~A}$ for this beam configuration since the interaction impedance for the $n=-1$ space harmonic is lower for the location outside the SWS. Simulations of the conventional BWO use only the beam outside the SWS while the simulations of the oscillator-amplifier use the additional solid beam at the center of the helix for forward-wave amplification. The solid beam at the center of the helix is considerd to have a beam current of $0.15 \mathrm{~A}$. Focusing magnetic field of $0.5 \mathrm{~T}$ is used for the simulations of both devices.

The time-domain output signal of both conventional BWO and oscillator-amplifier with a $4 \mathrm{KV}$ beam for the backward-wave interaction is shown in Fig. 6.14. As shown in the figure, the output signal grows smoothly with time and settles to a steady value for both devices. The DFT of the output signal from the oscillator-amplifier with two different beam voltages for the beam outside the SWS is shown in Fig. 6.15; the frequency of the output 
signal changes from $14.1 \mathrm{GHz}$ to $17.2 \mathrm{GHz}$ as the beam voltage changes from $4 \mathrm{KV}$ to 6 $\mathrm{KV}$.

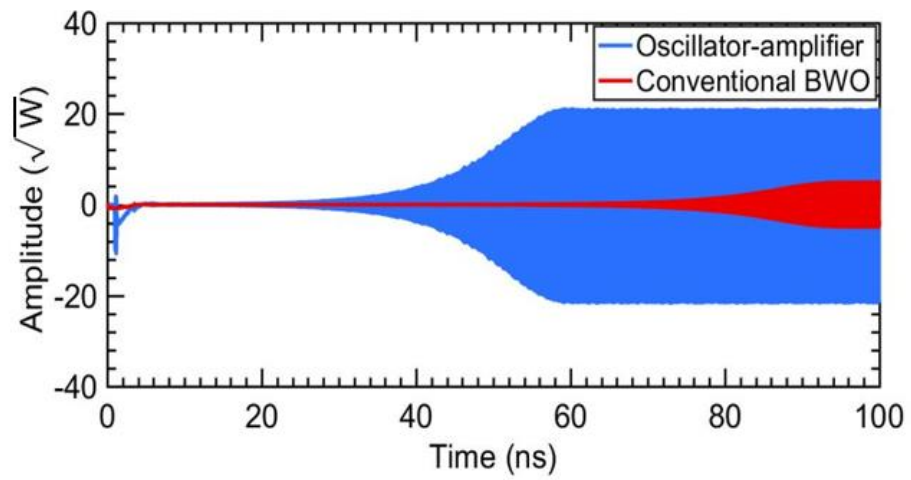

Figure 6.14:Output signal from the oscillator-amplifier and the conventional BWO. The oscillator-amplifier has a $4 \mathrm{KV}$ solid beam outside the SWS and a $17.2 \mathrm{KV}$ solid beam inside the SWS ( $3^{\text {rd }}$ beam configuration) whereas the conventional BWO has a $4 \mathrm{KV}$ solid beam outside the SWS.

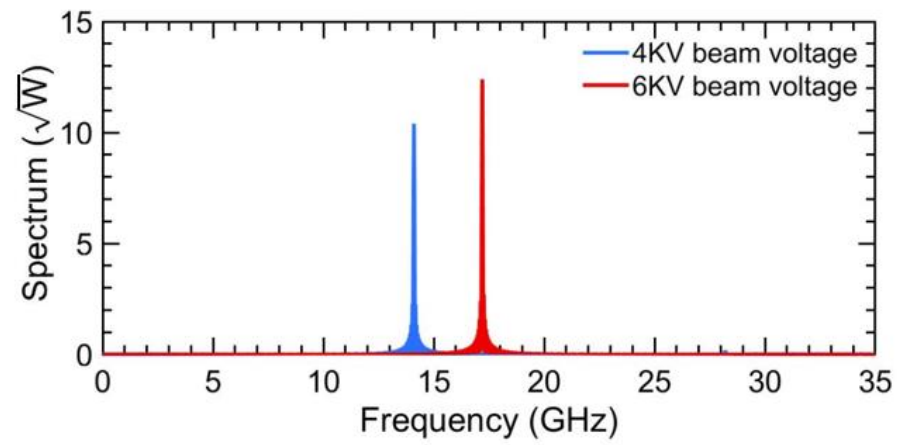

Figure 6.15: Discrete Fourier transform of the output signal from the oscillator-amplifier at two different beam voltages for the beam outside the SWS ( $3^{\text {rd }}$ beam configuration).

The performance of the conventional BWO and the oscillator-amplifier with the $3^{\text {rd }}$ beam configuration is also tabulated in Table 6.2. The BWO with this beam configuration has low efficiency since it uses an electron beam outside the SWS. In this beam configuration, the DC-to-RF conversion efficiency of the oscillator-amplifier is improved compared to that of the conventional BWO by a factor of six or more.

\subsection{Design of BWO and Oscillator-Amplifier Using PH-SEC}

For assessing the feasibility of the two-beam oscillator-amplifier with a planar SWS, the device is designed using the PH-SEC SWS. A conventional BWO is also designed to enable a comparison of the performance of the two devices. The following sub-sections 
present the structure, dimensions, dispersion characteristics, and interaction impedance of the PH-SEC SWS. The S-parameters of the SWS for both BWO and oscillator-amplifier are also presented. Only one beam configuration is used for both devices in this case.

\subsubsection{Design of PH-SEC}
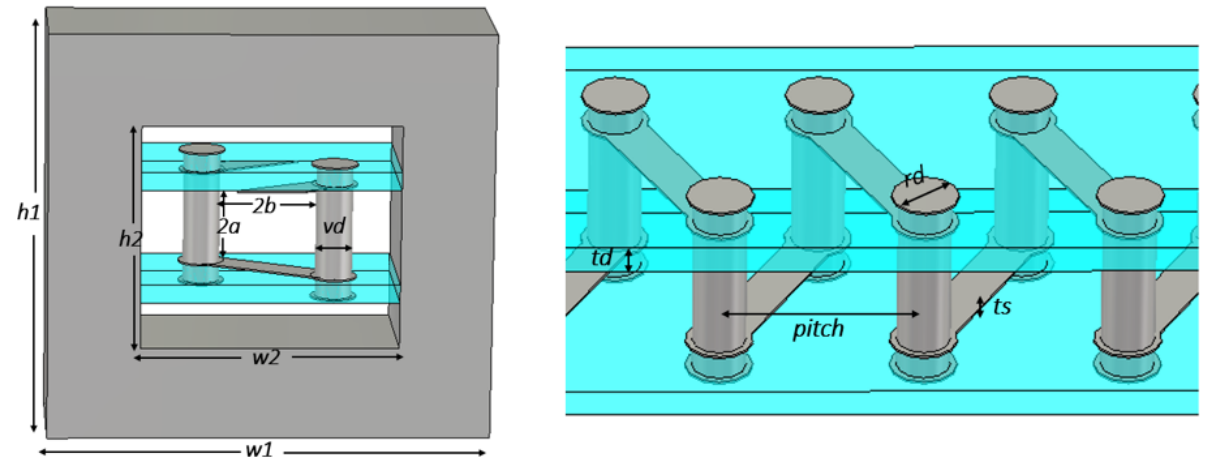

Figure 6.16:Cross-sectional and the perspective view of the PH-SEC.

Table 6.3 Dimensions of the PH-SEC.

\begin{tabular}{|c|c|c|c|}
\hline Parameter & Value $(\mathrm{mm})$ & Parameter & Value $(\mathrm{mm})$ \\
\hline $2 a$ & 1 & pitch & 1.5 \\
\hline $2 b$ & 1 & $h 2$ & 2.44 \\
\hline$r d$ & 0.5 & $h 1$ & 4.44 \\
\hline$v d$ & 0.4 & $w 2$ & 2.8 \\
\hline$t s$ & 0.017 & & \\
\hline
\end{tabular}

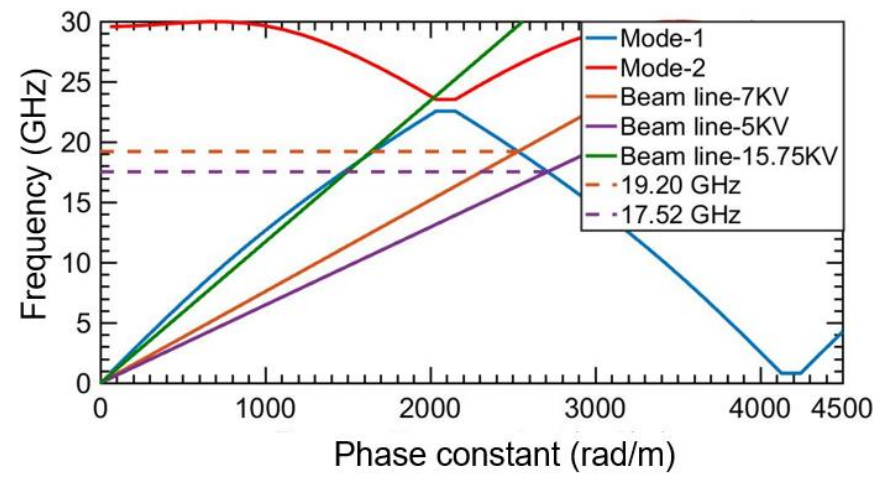

Figure 6.17: Dispersion characteristics of the SWS; also shown are three different beam lines.

Similar to the circular helix in the previous sections, the PH-SEC is also designd to operate at Ku-band. As shown in Fig. 6.16, the PH-SEC is supported by two identical 
dielectric substrates inside a metal enclosure. Straight-edge connections of the PH-SEC consist of copper wires of diameter $0.5 \mathrm{~mm}$. The top horizontal strips of the PH-SEC are printed on the upper dielectric substrate while the bottom horizontal strips are printed on the lower dielectric substrate. The design considers RO4003 substrates with thickness of $0.203 \mathrm{~mm}$, dielectric constant of 3.55, and loss tangent of 0.0027 . The dimensions of the SWS are tabulated in Table 6.3.

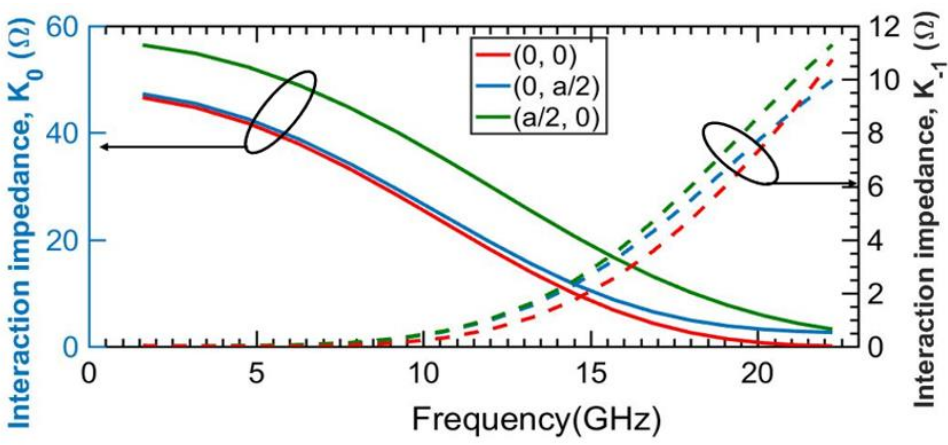

Figure 6.18: Interaction impedance of the fundamental $\left(\mathrm{K}_{0}\right)$ and $\mathrm{n}=-1\left(\mathrm{~K}_{-1}\right)$ space harmonic of the PH-SEC at three different beam positions; $(\mathrm{x}, \mathrm{y})$ are the coordinates of the beam position.

The dispersion characteristics of the SWS obtained using the CST eigenmode solver are shown in Fig. 6.17. The figure contains the phase constant of the fundamental mode and the first higher order mode of the SWS. Three beam lines corresponding to $5 \mathrm{KV}, 7$ $\mathrm{KV}$ and $15.75 \mathrm{KV}$ beam voltages are also included in the figure. The beam lines corresponding to $5 \mathrm{KV}$ and $7 \mathrm{KV}$ beam voltage intersect with the $\mathrm{n}=-1$ space harmonic of the fundamental mode at $17.5 \mathrm{GHz}$ and $19.2 \mathrm{GHz}$, respectively. These intersections indicate that the electron beam with $5 \mathrm{KV}$ and $7 \mathrm{KV}$ beam voltages can generate an EM signal of frequency around $17.5 \mathrm{GHz}$ and $19.2 \mathrm{GHz}$, respectively. The beam line corresponding to 15.75 KV beam voltage runs very close to the curve for the fundamental space harmonic of mode- 1 . This indicates that the $15.75 \mathrm{KV}$ beam voltage can amplify a wide range of frequencies including $17.5 \mathrm{GHz}$ and $19.2 \mathrm{GHz}$.

The interaction impedance versus frequency for both the fundamental $\left(\mathrm{K}_{0}\right)$ and the $\mathrm{n}=-1\left(\mathrm{~K}_{-1}\right)$ space harmonic of mode-1 at three different beam positions is shown in Fig. 6.18. Similar to the circular helix, the fundamental space harmonic has a higher interaction impedance than that of the $n=-1$ space harmonic. For a given beam position, the values of $\mathrm{K}_{0}$ decrease while that of $\mathrm{K}_{-1}$ increase as the frequency increases. The interaction 
impedance for both space harmonics increases when we move the beam position closer to the horizontal strips. The values of $\mathrm{K}_{0}$ and $\mathrm{K}_{-1}$ increase when we move the beam position closer to the straight-edge connections.

\subsubsection{S-parameters of the BWO and oscillator-amplifier}

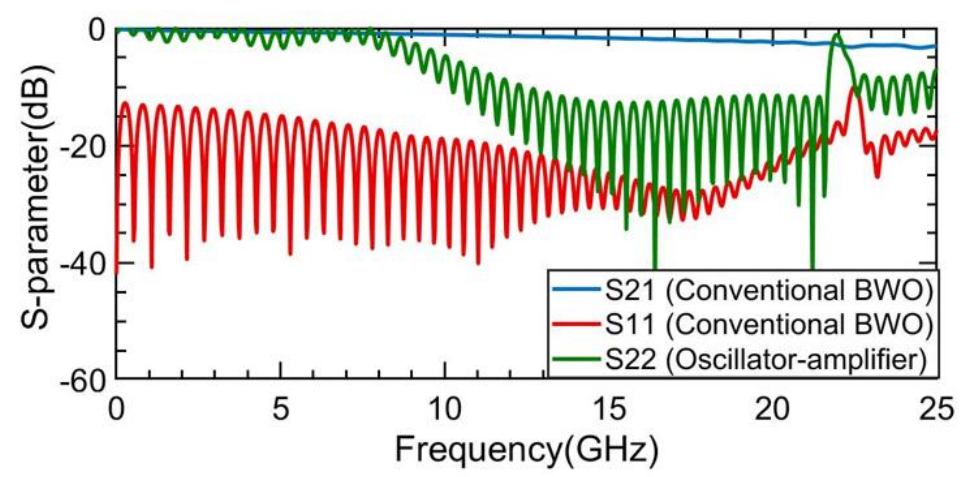

Figure 6.19: S-parameters of the PH-SEC SWS used as BWO and as oscillator-amplifier.

Similar to the BWO and oscillator-amplifier using the circular helix, here also fifty periods of the PH-SEC shown in Fig. 6.16 form the SWS for both devices. Hence, we keep a similar interaction length for both devices using either the circular helix or the PH-SEC. Both port-1 and port- 2 of the SWS are considered to be matched in simulations for the BWO. Port-1 is considered to be a perfect open-circuit and port-2 is matched in the simulations for the oscillator-amplifier. Similar to the SWS in Sub-section 5.4.1, here also CPW feedlines are designed at both ports of the BWO and one port of the oscillatoramplifier. Besides, pitch tapering is used at both ends of the SWS for getting a better match.

The S-parameters of both devices from simulation are shown in Fig. 6.19. The BWO has an S1 1 less than $-22 \mathrm{~dB}$ and S21 better than $-2.5 \mathrm{~dB}$ for the $14-20 \mathrm{GHz}$ frequency range. This frequency range represents a bandwidth of $35.3 \%$ and can be considered to be the expected bandwidth of operation of the BWO. The proposed oscillator-amplifier is a one port device and it has an S22 only better than $-11 \mathrm{~dB}$ for frequencies from $14-20 \mathrm{GHz}$. The complete mismatch at port-1 is the reason for this poor reflection characteristic of the oscillator-amplifier.

\subsubsection{Beam Configuration}

In this case, only one beam configuration is used for studying the characteristics of the BWO and the oscillator-amplifier. Fig. 6.20 shows the position of beams for the BWO and the oscillator-amplifier. Similar to the $2^{\text {nd }}$ beam configuration of oscillator-amplifier 
using circular helix, here also we use two solid cylindrical beams inside the SWS. The beams have a radius of $0.1 \mathrm{~mm}$ and are separated by a distance of $0.2 \mathrm{~mm}$. As shown in the figure, the upper beam is used for the backward-wave interaction while the lower beam is used for the forward-wave interaction. Simulations of the BWOs use only the beam for backward-wave interaction. The shape, position, and size of this beam is kept the same for the oscillator-amplifier.

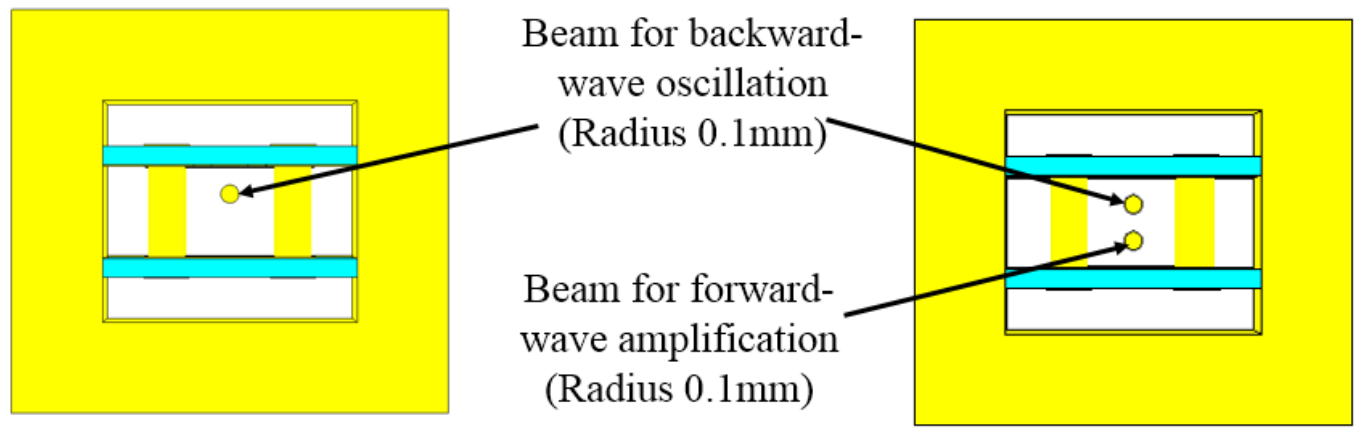

Figure 6.20: Position of E-beams for the BWO and the oscillator-amplifier.

\subsection{PIC Simulation Results}

Both conventional BWO and oscillator-amplifier are simulated using the PIC solver. The beam current for the BWO is fixed as $0.25 \mathrm{~A}$. Simulations of the oscillator-amplifier use an additional solid beam with beam current of $0.15 \mathrm{~A}$ for the forward-wave amplification. Both devices use a focusing magnetic field of $0.55 \mathrm{~T}$.

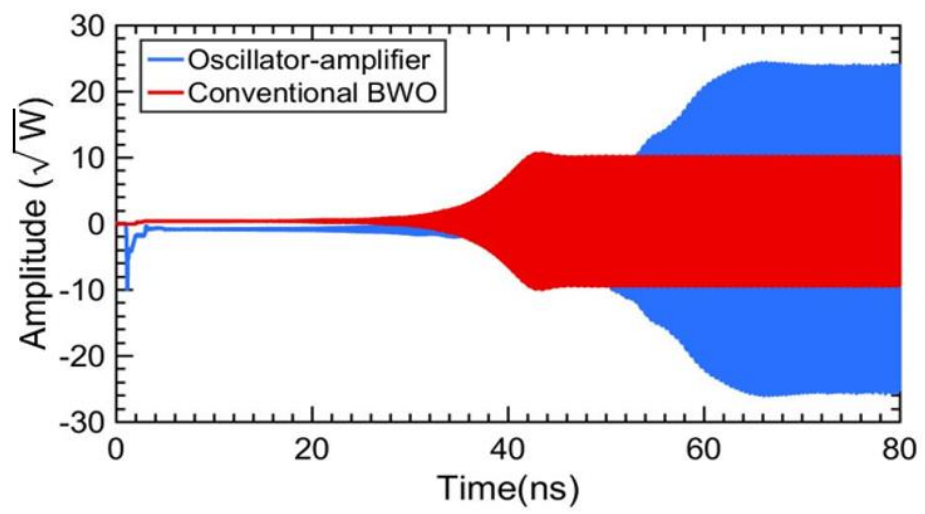

Figure 6.21: Output signal from the oscillator-amplifier with $5 \mathrm{KV}$ and $15.75 \mathrm{KV}$ solid beams, and the output signal from the BWO with $5 \mathrm{KV}$ solid beam.

The simulated time-domain output signal from the oscillator-amplifier using a $5 \mathrm{KV}$ beam for the backward-wave interaction and a $15.75 \mathrm{KV}$ beam for the forward-wave interaction is shown in Fig. 6.21. The time-domain output signal for the BWO with a $5 \mathrm{KV}$ 
beam is also shown in this figure. Here also the output signals from both devices grow with time and settle to a steady value. The amplitude of the output signal from the oscillatoramplifier is 2.5 times higher than that from the conventional BWO. The dicrete Fourier transform of the output signal from the oscillator-amplifier at beam voltages of 5 and $7 \mathrm{KV}$ for backward-wave interaction is shown in Fig. 6.22.

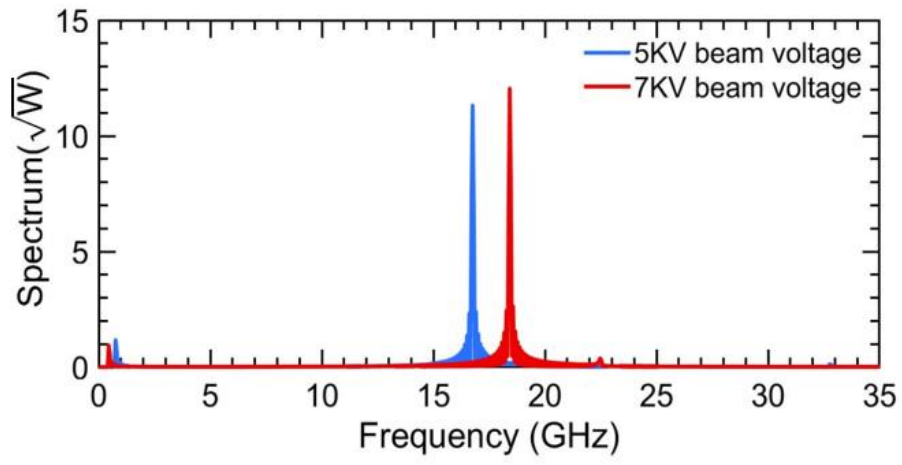

Figure 6.22: Discrete Fourier transform of the output signal from the oscillator-amplifier at two different beam voltages for backward-wave interaction.

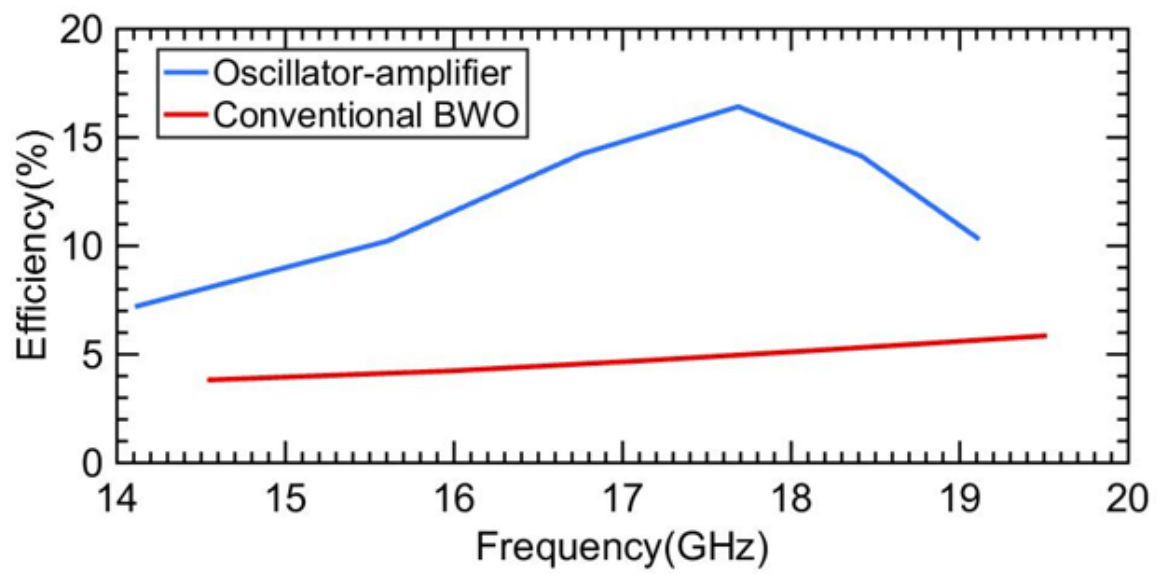

Figure 6.23: Comparison of efficiency of the oscillator-amplifier and the conventional BWO.

The tuneable bandwidth of the oscillator-amplifier is studied by varying the the beam voltage for the backward-wave interaction from $3 \mathrm{KV}$ to $8 \mathrm{KV}$ while keeping the beam voltage for the forward-wave interaction constant. Similarly, the tuneable bandwidth of the BWO is also studied by varying the the beam voltage from $3 \mathrm{KV}$ to $8 \mathrm{KV}$. The frequency of the output signal from the oscillator-amplifier varies from $14.1 \mathrm{GHz}$ to $19.1 \mathrm{GHz}$ while the output frequency of the BWO varies from $14.5 \mathrm{GHz}$ to $19.5 \mathrm{GHz}$. Both devices have a bandwidth of $5 \mathrm{GHz}$, showing clearly that the oscillator-amplifier does not compromise the 
tuneable bandwidth of the BWO. For a given beam voltage for the backward-wave interaction the frequency of the output signal from the oscillator-amplifier has a frequency deviation of $0.4 \mathrm{GHz}$ with respect to that from the conventional BWO. This deviation occurs due to the change in the dispersion characteristics of the oscillator-amplifier with the insertion of the beam for the forward-wave interaction.

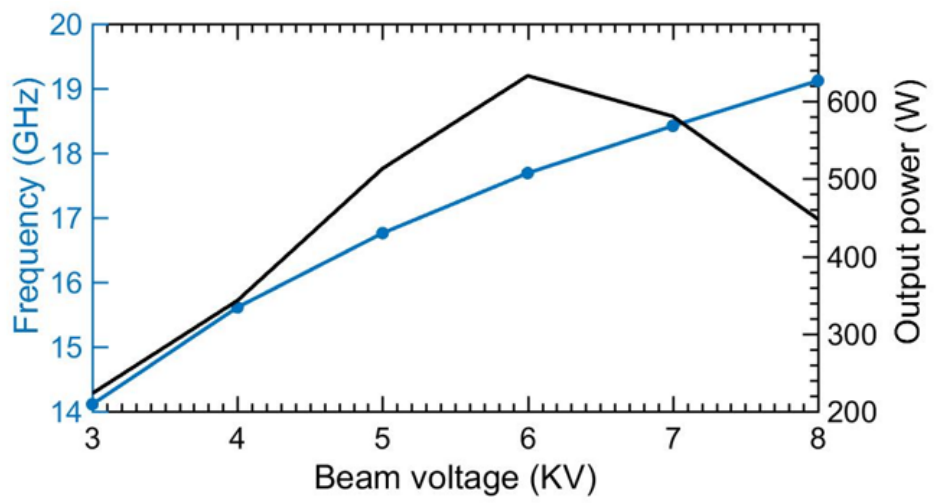

Figure 6.24: Variation of frequency and power of the output signal with beam voltage for the backward-wave interaction in the oscillator-amplifier.

The plot of efficiency versus frequency for both devices is given in Fig. 6.23. As shown in the figure, the oscillator-amplifier has an efficiency improvement of more than 2 times compared to that of the BWO over the entire frequency range. The oscillatoramplifier gives a maximum efficiency improvement of 3 times compared to that of the BWO. The power and frequency of the output signal from the oscillator-amplifier versus beam voltage for backward-wave interaction are shown in Fig. 6.24. The figure shows that the oscillator-amplifier can generate a maximum output power of $633 \mathrm{~W}$.

Like the circular helix, the interaction impedance for the PH-SEC is higher at locations closer to the inclined strips. Thus, higher efficiency can be obtained by placing the beam closer to the inclined strips, but this will increase the risk of beam collision with the SWS. Hence, we need to place the beam keeping in mind the beam parameters, interaction length, and strength of the magnetic field.

For the oscillator-amplifier, we have proposed the use of 2 beams at two different voltages to improve the efficiency of a conventional BWO. The beams can be generated using two different electron guns. Therefore, in principle, different cathode temperatures, beam voltages and beam currents can be achieved. Simulations show that a sufficiently 
high magnetic field can allow the transport of both electron beams through the SWS without any interference.

Generation of two electron beams with different beam voltage and current is challenging, but two electron beams with different beam voltages have been practically realized in the past in electron wave tubes [115]- [116].

\subsection{Summary}

This chapter presents a new technique to improve the efficiency and output power of the conventional non-relativistic BWOs. The new technique combines the operation of both oscillator and amplifier in a single SWS. An additional electron beam which is synchronized with the fundamental forward-wave space harmonic of the SWS is introduced in the conventional BWO. The additional beam provides amplification of the RF signal generated in the device due to backward-wave oscillation. The device resulting from the new technique is named as two-beam oscillator-amplifier. The overall efficiency of the oscillator-amplifier is a combination of the efficiency of both BWO and travelling-wave tube amplifier.

The technique is illustrated first using a circular helix SWS operating at Ku-band. Both a conventional BWO and an oscillator-amplifier are designed using the same SWS interaction length. Three different beam configurations are considered for studying the performance of the oscillator-amplifier using the PIC solver. The results show that the oscillator-amplifier improves the DC-to-RF conversion efficiency of the conventional BWO by a factor ranging from three to six depending on the beam configuration. The results show that the oscillator-amplifier gives a maximum DC-to-RF conversion efficiency of $16.8 \%$ and a peak output power of $625 \mathrm{~W}$.

The technique is also illustrated using the PH-SEC SWS at Ku-band. Both oscillatoramplifier and BWO are designed using the PH-SEC and the simulation results are compared. The results show that the oscillator-amplifier improves the DC-to-RF conversion efficiency of the BWO by a factor of two or more. A maximum efficiency improvement of three times is obtained from the oscillator-amplifier with PH-SEC SWS. Both BWO and the oscillator-amplifier show a tuneable bandwidth of $5 \mathrm{GHz}$. The results show that the oscillator-amplifier gives a maximum DC-to-RF conversion efficiency of $16.4 \%$ and a peak output power of $633 \mathrm{~W}$. These performance figures are very similar to those obtained when the circular helix is used as the SWS 
The proposed technique does not compromise the electronic tuneable bandwidth of the conventional BWO. Neither does it need an additional SWS. An extra electron beam is the only additional requirement for this technique. The oscillator-amplifier has a good potential for applications since it offers significant improvement in the efficiency and output power compared to a conventional nonrelativistic BWO. 


\section{Chapter 7 Conclusion and Future Work}

\subsection{Conclusion}

This thesis considers the application of planar helix slow-wave structure with straight-edge connections (PH-SEC) for millimetre wave backward-wave oscillators (BWOs). Microfabrication-compatible SWSs such as PH-SEC are essential for realizing high frequency travelling-wave tubes (TWTs). PH-SEC has been extensively studied for applications in travelling-wave tube amplifiers (TWTAs). These studies have proved that this structure is microfabrication-compatible with good thermal dissipation properties. The PH-SEC retains the wide bandwidth and strong beam-wave interaction properties of the popular circular helix slow-wave structure (SWS).

Although many studies have been conducted for PH-SEC, some significant aspects have not been investigated yet. First of all, the strength of different space harmonics in the PH-SEC has not been studied yet. Secondly, a field-theory based analysis for the accurate evaluation of dispersion characteristics of the PH-SEC has not been developed yet. Thirdly, the performance of a BWO with PH-SEC as the SWS has not been investigated yet. Finally, some method to improve the efficiency and output power of BWOs without compromising its tuneable bandwidth has not been developed yet.

In this thesis, first the Fourier decomposition method has been used to determine the interaction impedance of the fundamental as well as the non-fundamental space harmonics of a periodic SWS. The Fourier decomposition method allows to determine the magnitude of longitudinal electric field for different space harmonics from the total longitudinal electric field which is obtained from simulations. The magnitude of longitudinal electric field for different space harmonics so determined has been compared with those from the well-known tape-helix analysis. The comparison concludes that the tape-helix analysis fails to predict the magnitude of longitudinal electric field for non-fundamental space harmonics since the current assumption in the analysis does not consider the effect of non-fundamental space harmonics. Illustrative simulation results have been presented for the interaction impedance of the circular helix for the fundamental and $n=-1$ space harmonic at axial and different off-axis positions. This method has been used for evaluating the interaction impedance of various space harmonics of the PH-SEC in this thesis. Moreover, this method 
can be used for finding the interaction impedance of the desired space harmonic at desired beam position in any periodic SWS.

The dispersion characteristics and the interaction impedance of the fundamental space harmonic of the PH-SEC have been determined using the tape-helix approximation. The tape-helix analysis of an infinitely wide planar helix and the effective dielectric method (EDC) have been used for getting the characteristics of PH-SEC. It has been shown that the analytical results for the phase velocity and interaction impedance for a PH-SEC immersed in a homogeneous medium match very well with the simulation results. Besides, the results have greater accuracy than that from the sheath-helix analysis of PH-SEC. The tape-helix analysis has also been extended to determine the dispersion characteristics and interaction impedance of the fundamental space harmonic of the PH-SEC put inside a metal enclosure. These results also show a very good match with the simulation results. In addition, the tapehelix analysis has also been used to find the dispersion characteristics of the rectangular tape-helix. The results have been compared with the HFSS simulation result and the tapehelix analysis of rectangular helix in the literature. It has been shown that the results from the present analysis are more accurate over a broader bandwidth than the results from the complicated tape-helix analysis in the literature.

As the next step, PH-SEC immersed in a homogeneous medium has been fabricated and measured as an experimental validation of the tape-helix analysis. The measured phase velocity of the SWS shows a good match with that from the analysis and simulation over a frequency range 2 - $5 \mathrm{GHz}$.

The microfabrication-compatible PH-SEC SWS has been used to design a BWO operating at W-band. The results from particle-in-cell (PIC) simulations have been presented to show that the BWO can generate signals with frequency from $86.9 \mathrm{GHz}$ to 100 $\mathrm{GHz}$ with a tuneable bandwidth of $14 \%$. The performance of the oscillator has been presented for three different beam currents. It has been shown that the designed oscillator can generate a peak output power of $2.3 \mathrm{~W}$ and it can give a maximum DC-to-RF efficiency of $1.62 \%$. A scaled version of the PH-SEC to operate at X-band frequencies has been designed and fabricated using printed-circuit technique to validate the accuracy of simulation. The measured S-parameters and phase velocity of the SWS show a good match with the simulation results. 
Finally, a new device named two-beam oscillator-amplifier has been proposed to improve the efficiency and output power of the conventional non-relativistic BWO. The oscillator-amplifier combines the operation of both oscillator and amplifier in a single SWS Hence it does not affect the size of the conventional BWO. It does not compromise the electronic tuneable bandwidth of the conventional BWO either. The advantages of the new device have been illustrated by designing and simulating both conventional BWO and oscillator-amplifier using the circular helix SWS. It has been shown that the oscillatoramplifier improves the DC-to-RF conversion efficiency of the conventional BWO by a factor ranging from three to six depending on the beam configuration. The oscillatoramplifier has also been illustrated using the PH-SEC SWS. It has been shown that the oscillator-amplifier using the PH-SEC can improve the DC-to-RF conversion efficiency of the BWO by a factor of two or more.

\subsection{Recommendations for Further Research}

This thesis studies the application of PH-SEC in BWOs. The thesis also presents an accurate method to estimate the interaction impedance of different space harmonics of a periodic SWS. Finally, the thesis presents a new technique to improve the low efficiency of conventional BWOs. There are some possible extensions of the studies presented in this thesis. These are mentioned below.

1) The analysis of PH-SEC using tape-helix approximation in Chapter 3 considers the structure in the presence of a homogeneous medium. This is not a practical situation. A more practical configuration of the PH-SEC, such as the helix with beam tunnel in the presence of multiple dielectric layers can be considered. Results from such analysis can be used for more accurate selection of the initial dimensions of the PH-SEC for a TWT with given target specifications.

2) Even though the previous studies show that the PH-SEC has very good thermal dissipation properties, the thermal dissipation in a BWO with PH-SEC has not been studied. Attempt should be made to study the thermal characteristics of a BWO based on PH-SEC. The thermal dissipation properties are important to assess the maximum output power from such a device.

3) Attempts should be made to microfabricate and measure the properties of a BWO similar to that in Chapter 5. Hot-test characteristics of the BWO should be measured. Otherwise, a version of the BWO scaled down in frequency can be 
fabricated using printed circuit technique and its hot-test characteristics can be measured.

4) The PH-SEC based BWO and travelling-wave tube amplifier (TWTA) can be connected together to realize a high frequency high power signal generator. Both devices can also be fabricated together since they have similar fabrication steps. Work can be carried out on studying the performance of a system which contains both TWTA and BWO.

5) Experimental validation of the two-beam oscillator-amplifier in Chapter 6 has not been carried out yet. Work can be carried out on fabricating and measuring the performance of the oscillator-amplifier since the simulations show that the device promises to give a good output performance. 



\section{Author's Publications}

1. A. Kumar. M. M., S. Aditya and C. Chua, "Interaction Impedance for Space Harmonics of Circular Helix Using Simulations," IEEE Transactions on Electron Devices, vol. 64, no. 4, pp. 1868-1872, April 2017. doi: 10.1109/TED.2017.2669332

2. A. Kumar M. M and S. Aditya, "Vacuum Electronic Two-Beam OscillatorAmplifier," IEEE Transactions on Plasma Science, vol. 45, no. 8, pp. 2260-2267, Aug. 2017. doi: 10.1109/TPS.2017.2726109

3. A. Kumar M. M and S. Aditya, "Simplified Tape-Helix Analysis of the Planar Helix Slow Wave Structure with Straight-Edge Connections," IEEE Transactions on Electron Devices, vol. 65, no. 6, pp. 2280-2286, June 2018. doi: 10.1109/TED.2018.2797928

4. M. M. Ajith Kumar, S. Aditya and S. Wang, "A W-Band Backward-Wave Oscillator Based on Planar Helix Slow Wave Structure," IEEE Transactions on Electron Devices, vol. 65, no. 11, pp. 5097-5102, Nov. 2018. doi: 10.1109/TED.2018.287178

5. M. M. Ajith Kumar, Chen Zhao, Shaomeng Wang and S. Aditya, "Backward wave oscillator using a planar helix slow-wave structure with straight-edge connections," 2016 IEEE International Vacuum Electronics Conference (IVEC), Monterey, CA, 2016, pp. 1-2. doi: 10.1109/IVEC.2016.7561902

6. M. M. A. Kumar and S. Aditya, "Simplified tape-helix analysis of planar helix slowwave structure using effective dielectric constant method," 2017 Eighteenth International Vacuum Electronics Conference (IVEC), London, 2017, pp. 1-2. doi: 10.1109/IVEC.2017.8289700

7. M. M. A. Kumar, S. Aditya and C. Zhao, "Transmission characteristics of planar tape-helix: Simulation and measurements," 2018 IEEE International Vacuum Electronics Conference (IVEC), Monterey, CA, USA, 2018, pp. 343-344. doi: 10.1109/IVEC.2018.8391517

8. M. M. A. Kumar and S. Aditya, "Dispersion characteristics of planar tape-helix using effective dielectric constant method," 2018 IEEE International Vacuum Electronics Conference (IVEC), Monterey, CA, USA, 2018, pp. 381-382. doi: 10.1109/IVEC.2018.8391683 
Bibliography

[1] R. J. Barker, J. H. Booske, N. C. Luhmann, and G. S. Nusinovich, "Modern microwave and millimeter-wave power electronics," Mod. Microw. MillimeterWave Power Electron., pp. 1-827, 2005.

[2] J. H. Booske, R. J. Dobbs, C. D. Joye, C. L. Kory, G. R. Neil, G.-S. Park, J. Park, and R. J. Temkin, "Vacuum Electronic High Power Terahertz Sources," IEEE Trans. Terahertz Sci. Technol., vol. 1, no. 1, pp. 54-75, Sep. 2011.

[3] R. H. Abrams, B. Levush, A. A. Mondelli, and R. K. Parker, "Vacuum electronics for the 21st century," IEEE Microw. Mag., vol. 2, no. 3, pp. 61-72, 2001.

[4] B. Gorshunov, A. Volkov, I. Spektor, A. Prokhorov, A. Mukhin, M. Dressel, S. Uchida, and A. Loidl, "Terahertz BWO-spectrosopy," Int. J. Infrared Millimeter Waves, vol. 26, no. 9, pp. 1217-1240, 2005.

[5] B. Gompf, N. Gebert, H. Heer, and M. Dressel, "Polarization contrast terahertz-nearfield imaging of anisotropic conductors," Appl. Phys. Lett., vol. 90, no. 8, 2007.

[6] J. Feng, Y. Tang, D. Gamzina, X. Li, B. Popovic, M. Gonzalez, L. Himes, R. Barchfeld, H. Li, P. Pan, R. Letizia, C. Paoloni, and N. C. Luhmann, "Fabrication of a 0.346-THz BWO for Plasma Diagnostics," IEEE Trans. Electron Devices, vol. 65, no. 6, pp. 2156-2163, 2018.

[7] J. W. Gewartowski and H. A. Watson, Principles of Electron Tubes. New York,USA: Van Nostrand, 1965.

[8] R. Kompfner and N. T. Williams, "Backward-Wave Tubes," Proc. IRE, vol. 41, no. 11, pp. 1602-1611, 1953.

[9] H. R. Johnson, "Backward-Wave Oscillators," Proc. IRE, vol. 43, no. 6, pp. 684697, 1955.

[10] J. A. Dayton, C. L. Kory, G. T. Mearini, D. Malta, M. Lueck, and B. Vancil, "Fabrication and testing of the $0.650 \mathrm{THz}$ helical BWO," 2012 IEEE 13th Int. Vac. Electron. Conf. IVEC 2012, pp. 33-34, 2012.

[11] L. Hu, R. Song, G. Ma, Y. Jiang, W. Lei, F. Meng, and H. Chen, "Experimental Demonstration of a $0.34-\mathrm{THz}$ Backward-Wave Oscillator with a Sinusoidally Corrugated Slow-Wave Structure," IEEE Trans. Electron Devices, vol. 65, no. 6, pp. 2149-2155, 2018.

[12] M. Mineo and C. Paoloni, "Corrugated rectangular waveguide tunable backward wave oscillator for terahertz applications," IEEE Trans. Electron Devices, vol. 57, no. 6, pp. 1481-1484, 2010.

[13] Z. Wang, Y. Gong, Y. Wei, Z. Duan, Y. Zhang, L. Yue, H. Gong, H. Yin, Z. Lu, J. $\mathrm{Xu}$, and J. Feng, "High-power millimeter-wave BWO driven by sheet electron beam," IEEE Trans. Electron Devices, vol. 60, no. 1, pp. 471-477, 2013.

[14] J. Lai, Y. Wei, X. Xu, Y. Liu, and F. Shen, “A 140GHz Staggered Double Vane Backward Wave Oscillator,” no. Gsmm, pp. 316-319, 2012.

[15] J. Cai, L. Hu, H. Chen, X. Jin, G. Ma, and H. Chen, "Study on the Increased Threshold Current in the Development of 220-GHz Folded Waveguide Backward- 
Wave Oscillator," IEEE Trans. Microw. Theory Tech., vol. 64, no. 11, pp. 36783685, 2016.

[16] J. S. Hummelt, S. M. Lewis, M. A. Shapiro, and R. J. Temkin, "Design of a metamaterial-based backward-wave oscillator," IEEE Trans. Plasma Sci., vol. 42, no. 4, pp. 930-936, 2014.

[17] Y. Wang, Z. Duan, F. Wang, S. Li, Y. Nie, Y. Gong, and J. Feng, "S-Band HighEfficiency Metamaterial Microwave Sources," IEEE Trans. Electron Devices, vol. 63, no. 9, pp. 3747-3752, 2016.

[18] X. Tang, Z. Duan, X. Ma, S. Li, F. Wang, Y. Wang, Y. Gong, and J. Feng, "Dual Band Metamaterial Cherenkov Oscillator with a Waveguide Coupler," IEEE Trans. Electron Devices, vol. 64, no. 5, pp. 2376-2382, 2017.

[19] A. I. Nashed, S. K. Chaudhuri, and S. Safavi-Naeini, "A 650-GHz backward wave oscillator based on axial loaded double defected-photonic crystal SWS," IEEE Trans. Plasma Sci., vol. 45, no. 3, pp. 372-380, 2017.

[20] A. I. Nashed, S. K. Chaudhuri, and S. Safavi-Naeini, "Analysis and design of a novel photonic crystal-based sub-mm/THz backward-wave oscillator," IEEE Trans. Terahertz Sci. Technol., vol. 2, no. 6, pp. 642-651, 2012.

[21] G. O. Vela, M. S. Miller, R. W. Grow, and J. M. Baird, "Terahertz Backward-wave Oscillators with Photonic Crystal Waveguides," 2006 IEEE Int. Vac. Electron. Conf. held Jointly with 2006 IEEE Int. Vac. Electron Sources, pp. 425-426, 2006.

[22] R. Letizia, M. Mineo, and C. Paoloni, "Photonic Crystal-Coupler for Sheet Beam THz Vacuum Electron Tubes," IEEE Electron Device Lett., vol. 37, no. 9, pp. 12271230, 2016.

[23] C. K. Chong and W. L. Menninger, "Latest advancements in high-power millimeterwave helix TWTs," IEEE Trans. Plasma Sci., vol. 38, no. 6 PART 1, pp. 1227-1238, 2010 .

[24] R. L. Ives, "Microfabrication of high-frequency vacuum electron devices," IEEE Trans. Plasma Sci., vol. 32, no. 3 I, pp. 1277-1291, 2004.

[25] Y. M. Shin, J. K. So, K. H. Jang, J. H. Won, a. Srivastava, S. T. Han, G. S. Park, J. H. Kim, S. S. Chang, R. K. Sharma, and S. N. Joshi, "Experimental Investigation of 95GHz Folded Waveguide Backward Wave Oscillator Fabricated by Two-Step LIGA," 2006 IEEE Int. Vac. Electron. Conf. held Jointly with 2006 IEEE Int. Vac. Electron Sources, pp. 419-420, 2006.

[26] S. T. Han, J. K. So, K. H. Jang, Y. M. Shin, J. H. Kim, S. S. Chang, N. M. Ryskin, and G. S. Park, "Investigations on a microfabricated FWTWT oscillator," IEEE Trans. Electron Devices, vol. 52, no. 5, pp. 702-708, 2005.

[27] S. Sengele, H. Jiang, J. H. Booske, C. L. Kory, D. W. van der Weide, and R. L. Ives, "Microfabrication and characterization of a selectively metallized w-band meanderline TWT circuit," IEEE Trans. Electron Devices, vol. 56, no. 5, pp. 730-737, 2009.

[28] Y. M. Shin, G. S. Park, G. P. Scheitrum, and B. Arfin, "Novel Coupled-Cavity TWT Structure Using Two-Step LIGA Fabrication," IEEE Trans. Plasma Sci., vol. 31, no. 6 II, pp. 1317-1324, 2003.

[29] C. Chua, J. M. Tsai, S. Aditya, M. Tang, S. W. Ho, Z. Shen, and L. Wang, 
"Microfabrication and characterization of W-band planar helix slow-wave structure with straight-edge connections," IEEE Trans. Electron Devices, vol. 58, no. 11, pp. 4098-4105, 2011.

[30] C. S. Chua, C. Zhao, and S. Aditya, "Design and Fabrication of a Planar Helix SlowWave Structure for C/X -Band TWT," IEEE Trans. Components, Packag. Manuf. Technol., vol. 7, no. 10, pp. 1663-1669, 2017.

[31] C. Chua, S. Aditya, and Z. Shen, "Effective dielectric constant method for a planar helix with straight-edge connections," IEEE Electron Device Lett., vol. 30, no. 11, pp. 1215-1217, 2009.

[32] C. Zhao, S. Aditya, S. Wang, J. Miao, and X. Xia, “A Wideband Microfabricated Ka-Band Planar Helix Slow-Wave Structure," IEEE Trans. Electron Devices, vol. 63, no. 7, pp. 2900-2906, 2016.

[33] S. Wang, S. Aditya, and S. Member, "Design of a Sheet-Beam Electron-Optical System for a Microfabricated W -Band Traveling-Wave Tube Using a Cold Cathode," IEEE Trans. Electron Devices, vol. 63, no. 9, pp. 3725-3732, 2016.

[34] J. Pasour, K. Nguyen, E. Wright, A. Balkcum, J. Atkinson, M. Cusick, and B. Levush, "Demonstration of a 100-kW solenoidally focused sheet electron beam for millimeter-wave amplifiers," IEEE Trans. Electron Devices, vol. 58, no. 6, pp. 1792-1797, 2011.

[35] A. S. Gilmour, Principles of Traveling Wave Tubes. .Boston, MA USA: Artech House, 1994, 1994.

[36] J. R. Pierce, “Traveling-Wave Tubes,” Bell Syst. Tech. J., vol. 29, no. 3, pp. 390$460,1950$.

[37] J. R. Pierce and L. M. Field, “Traveling-Wave Tubes," Proc. IRE, vol. 35, no. 2, pp. 108-111, 1947.

[38] J. R. Pierce, "Theory of the Beam-Type Traveling-Wave Tube," Proc. IRE, vol. 35, no. 2, pp. 111-123, 1947.

[39] H. J. Curnow, "A General Equivalent Circuit for Coupled-Cavity Slow-Wave Structures," IEEE Trans. Microw. Theory Tech., vol. 13, no. 5, pp. 671-675, 1965.

[40] T. M. Antonsen, A. N. Vlasov, D. P. Chernin, I. A. Chernyavskiy, and B. Levush, "Transmission line model for folded waveguide circuits," IEEE Trans. Electron Devices, vol. 60, no. 9, pp. 2906-2911, 2013.

[41] B. N. Basu., Electromagnetic theory and applications in beam-wave electronics. Singapore ; River Edge, N.J. : World Scientific, 1996.

[42] Robert E. Collin, Foundations for Microwave Engineering, $2^{\text {nd }}$ eidtion. N.Y:McGraw-Hill, 1992.

[43] S. Sensiper, "Electromagnetic Wave Propagation on Helical Structures (A Review and Survey of Recent Progress)," Proc. IRE, vol. 43, no. 2, pp. 149-161, 1955.

[44] A. W. Horsley and A. Pearson, "Measurement of Dispersion and Interaction Impedance Characteristics of Slow-Wave Structures by Resonance Methods," IEEE Trans. Electron Devices, no. 12, pp. 962-969, 1966.

[45] M. R. Amin, K. Ogura, J. Kojima, and R. H. Sagor, "Electromagnetic properties of a trapezoidally corrugated slow wave structure for backward wave oscillators," IEEE 
Trans. Plasma Sci., vol. 42, no. 6, pp. 1495-1501, 2014.

[46] P. Wang and R. G. Carter, "An Improved Technique for Measuring the Pierce Impedance of Helix Slow-Wave Structures," in 24th European Microwave Conference, Cannes, France, 1994, no. 1, pp. 998-1003.

[47] S. J. Rao, S. Ghosh, P. K. Jain, and B. N. Basu, "Nonresonant Perturbation measurements on dispersion and interaction impedance characteristics of helical slow-wave structures," IEEE Trans. Microw. Theory Tech., vol. 45, no. 9, pp. 15851593, 1997.

[48] C. W. Steele, “A Nonresonant Perturbation Theory," IEEE Trans. Microw. Theory Tech., vol. MTT-14, no. 2, pp. 70-74, 1966.

[49] D. A. Watkins and A. E. Siegman, "Helix impedance measurements using an electron beam," J. Appl. Phys., vol. 24, no. 7, pp. 917-922, 1953.

[50] N. E. Lindenbald, "Electron discharge device system," 2,300,052.

[51] R. Kompfner, "The Traveling-Wave Tube as Amplifier at Microwaves," Proc. IRE, vol. 35, no. 2, pp. 124-127, 1947.

[52] R. Kompfner, "The Invention of Traveling Wave Tubes," IEEE Trans. Electron Devices, vol. 23, no. 7, pp. 730-738, 1976.

[53] L. M. Field, "Some Slow-Wave Structures for Traveling-Wave Tubes," Proc. IRE, vol. 37, no. 1, pp. 34-40, 1949.

[54] H. Heffner, "Analysis of the backward-wave traveling-wave tube," Proc. IRE, vol. 42, no. 6, pp. 930-937, 1954.

[55] R. W. Grow and D. A. Watkins, "Backward-Wave Oscillator Efficiency," Proc. IRE, vol. 43, no. 7, pp. 848-856, 1955.

[56] L. R. Walker, "Starting currents in the backward-wave oscillator," J. Appl. Phys., vol. 24, no. 7, pp. 854-859, 1953.

[57] Y. H. Na, S. W. Chung, and J. J. Choi, "Analysis of a broadband Q band folded waveguide traveling-wave tube," IEEE Trans. Plasma Sci., vol. 30, no. 3 I, pp. 1017-1023, 2002.

[58] D. Pershing, K. Nguyen, D. K. Abe, E. Wright, P. Larsen, J. Pasour, S. Cooke, A. Balkcum, F. Wood, R. Myers, and B. Levush, "Demonstration of a wideband 10kW Ka-band sheet beam TWT amplifier," IEEE Int. Vac. Electron. Conf. IVEC 2014, pp. 121-122, 2014.

[59] C. Paoloni and M. Mineo, "Double corrugated waveguide for G-band traveling wave tubes," IEEE Trans. Electron Devices, vol. 61, no. 12, pp. 4259-4263, 2014.

[60] J. A. Dayton, G. T. Mearini, H. Chen, and C. L. Kory, "Diamond-studded helical traveling wave tube," IEEE Trans. Electron Devices, vol. 52, no. 5, pp. 695-701, 2005.

[61] X. Xu, Y. Wei, F. Shen, Z. Duan, Y. Gong, H. Yin, and W. Wang, "Sine waveguide for 0.22-THz traveling-wave tube," IEEE Electron Device Lett., vol. 32, no. 8, pp. 1152-1154, 2011.

[62] D. R. Gunderson, "Starting Conditions for Backward-Wave Oscillators with Large Loss and Large Space Charge," IEEE Trans. Electron Devices, vol. 17, no. 12, pp. 
1032-1039, 1970.

[63] M. I. Yalandin, V. G. Shpak, S. A. Shunailov, M. R. Oulmaskoulov, N. S. Ginzburg, I. V. Zotova, Y. V. Novozhilova, A. S. Sergeev, A. D. R. Phelps, A. W. Cross, S. Mark Wiggins, and K. Ronald, "Generation of powerful subnanosecond microwave pulses in the range of 38-150 ghz," IEEE Trans. Plasma Sci., vol. 28, no. 5, pp. 1607-1614, 2000.

[64] A. I. Klimov, S. D. Korovin, V. V. Rostov, M. R. Ulmaskulov, V. G. Shpak, S. A. Shunailov, and M. I. Yalandin, "Highly efficient generation of subnanosecond microwave pulses in Ka-band relativistic BWO," IEEE Trans. Plasma Sci., vol. 30, no. 3 I, pp. 1120-1125, 2002.

[65] C. Chen, G. Liu, W. Huang, Z. Song, J. Fan, and H. Wang, "A Repetitive X-Band Relativistic Backward-Wave Oscillator,” vol. 30, no. 3, pp. 1108-1111, 2002.

[66] D. M. Goebel, Y. Carmel, and G. S. Nusinovich, "Advances in plasma-filled microwave sources," Phys. Plasmas, vol. 6, no. 5 I, pp. 2225-2232, 1999.

[67] X. Renzhen, L. Yuzheng, S. Zhimin, C. Changhua, L. Guozhi, and A. T. Annular, "Theoretical Study of a Plasma-Filled Relativistic Cerenkov Generator With Coaxial Slow-Wave Structure,” vol. 35, no. 5, pp. 1456-1466, 2007.

[68] I. Introduction, "Experimental Study of a Plasma-Filled Backward Wave Oscillator," Science (80-. )., vol. 21, no. 1, pp. 142-150, 1993.

[69] D. S. B. Bwo, Y. Zhang, Y. Gong, Z. Wang, S. Liu, Y. Wei, Z. Duan, S. Member, X. Shi, Y. Wang, L. Zhang, Q. Zhou, and J. Liao, "Study of High-Power Ka-Band Rectangular Double-Grating Sheet Beam BWO," vol. 42, no. 6, pp. 1502-1508, 2014.

[70] U. Chipengo, M. Zuboraj, N. K. Nahar, and J. L. Volakis, "A Novel Slow-Wave Structure for High-Power Ka-Band Backward Wave Oscillators with Mode Control," IEEE Trans. Plasma Sci., vol. 43, no. 6, pp. 1879-1886, 2015.

[71] Y. Park, Y. Soga, M. Takeda, M. Sato, K. Kamada, T. Fujita, T. Okada, Y. Nishimura, and M. Yoshida, "Design and development of $90 \mathrm{GHz}$ backward-wave oscillator with staggered double-vane slow-wave structure," 2016 IEEE Int. Vac. Electron. Conf. IVEC 2016, pp. 4-5, 2016.

[72] C. Paoloni, D. Gamzina, L. Himes, B. Popovic, R. Barchfeld, L. Yue, Y. Zheng, X. Tang, Y. Tang, P. Pan, H. Li, R. Letizia, M. Mineo, J. Feng, and N. C. Luhmann, "THz Backward-Wave Oscillators for Plasma Diagnostic in Nuclear Fusion," IEEE Trans. Plasma Sci., vol. 44, no. 4, pp. 369-376, 2016.

[73] X. Xu, Y. Wei, F. Shen, T. Tang, H. Gong, W. Wang, and Y. Gong, "Sine waveguide with a grating reflector for 1-THz backward wave oscillator," 2012 IEEE 13th Int. Vac. Electron. Conf. IVEC 2012, pp. 399-400, 2012.

[74] K. Swaminathan, C. Zhao, C. Chua, and S. Aditya, "Vane-loaded planar helix slowwave structure for application in broadband traveling-wave tubes," IEEE Trans. Electron Devices, vol. 62, no. 3, pp. 1017-1023, 2015.

[75] C. Zhao, S. Aditya, S. Wang, and C. Chua, "A wideband planar helix slow-wave structure for millimeter-wave TWTs," Proc. 2015 IEEE Int. Vac. Electron. Conf. IVEC 2015, pp. 1-2, 2015. 
Bibliography

[76] C. Zhao, S. Aditya, and C. Chua, "Connected pair of planar helices with straightedge connections for application in TWTs," IEEE Trans. Electron Devices, vol. 61, no. 6, pp. 1692-1698, 2014.

[77] “Computer Simulation Technology,” [Online]. Available: http://www.cst.com. .

[78] "Ansoft HFSS user guide, Ansoft Corp," [Online]. Available: http://www.ansoft.com/products/electronics/ansoft-hfss. .

[79] "FEKO - EM Simulation Software.," "[Online]. Available: https://www.feko.info/. .

[80] and A. J. J. Petillo, A. Mankofsky, W. A. Krueger, C. Kostas, A. A. Mondeli and T. Drobot, "Applications of the ARGUS code in accelerator physics," Proc. Comput. Accel. Phys. Conf., pp. 303-312, 1994.

[81] "MAGIC. Alliant Techsystems Operations LLC," [Online]. Available: http://www.mrcwdc.com/magic/description.html. .

[82] D. K. Abe, B. Levush, T. M. Antonsen, D. R. Whaley, and B. G. Danly, "Design of a linear C-band helix TWT for digital communications experiments using the Christine suite of large-signal codes," IEEE Trans. Plasma Sci., vol. 30, no. 3 I, pp. 1053-1062, 2002.

[83] C. Nieter and J. R. Cary, "VORPAL: A versatile plasma simulation code," J. Comput. Phys., vol. 196, no. 2, pp. 448-473, 2004.

[84] S. Sengele, M. L. Barsanti, T. A. Hargreaves, C. M. Armstrong, J. H. Booske, and Y. Y. Lau, "Backward-wave suppression analysis, and design and fabrication of a prototype millimeter-wave ring-bar slow-wave structure," IEEE Trans. Plasma Sci., vol. 42, no. 12, pp. 3949-3960, 2014.

[85] C. Fu, Y. Wei, W. Wang, and Y. Gong, "Dispersion characteristics of a rectangular helix slow-wave structure," IEEE Trans. Electron Devices, vol. 55, no. 12, pp. 35823589, 2008.

[86] W. Wei, Y. Wei, W. Wang, M. Zhang, H. Gong, and Y. Gong, "Dispersion equations of a rectangular tape helix slow-wave structure," IEEE Trans. Microw. Theory Tech., vol. 63 , no. 5 , pp. 1445-1456, 2015.

[87] M. Chodorow and E. L. Chu, "Cross-wound twin helices for traveling-wave tubes," J. Appl. Phys., vol. 26, no. 1, pp. 33-43, 1955.

[88] D. T. Lopes and C. C. Motta, "Characterization of ring-bar and contrawound helix circuits for high-power traveling-wave tubes," IEEE Trans. Electron Devices, vol. 55, no. 9, pp. 2498-2504, 2008.

[89] B. J. Mcmurtry, "Fundamental Interaction Impedance of a Helix Surrounded by a Dielectric and a Metal Shield," IRE Trans. Electron Devices, vol. 9, no. 2, pp. 210216, 1962.

[90] P. K. Tien, “Traveling-Wave Tube Helix Impedance," Proc. IRE, vol. 41, no. 11, pp. 1617-1623, 1953.

[91] P. K. Jain and B. N. Basu, "The Inhomogeneous Dielectric Loading Effects of Practical Helix Supports on the Interaction Impedance of the Slow-Wave Structure of a TWT," IEEE Trans. Electron Devices, vol. 39, no. 3, pp. 727-733, 1992.

[92] A. K. Sinha, V. R, R. K. Gupta, K. L, J. S. N, J. P. K, and B. B. N, "Simplified tape model of arbitrarily-loaded helical slow-wave structures of a travelling-wave tube," 
vol. 139, no. 4, pp. 347-350, 1992.

[93] Y. Zhang, Y. L. Mo, J. Q. Li, and X. L. Zhou, "Modelling of finite size vane-loaded helical slow-wave structures," IEE Proc. - Microwaves, Antennas Propag., vol. 151, no. 2, p. 135, 2004.

[94] A. K. Varshney, R. Guha, S. K. Ghosh, and B. N. Basu, "Gain-frequency response of a helix traveling-wave tube with T-shaped dielectric support rods in a metal envelope," J. Electromagn. Waves Appl., vol. 30, no. 5, pp. 566-578, 2016.

[95] C. L. Kory and J. A. Dayton, "Computational investigation of experimental interaction impedance obtained by perturbation for helical traveling-wave tube structures," IEEE Trans. Electron Devices, vol. 45, no. 9, pp. 2063-2071, 1998.

[96] C. Fu, Y. Wei, Y. Yang, Y. Ju, X. Wang, D. Yang, and X. He, "Simulation of Rectangular Helix Slow-Wave Structure for $140 \mathrm{GHz}$ Traveling-Wave Tube," IEEE Trans. Plasma Sci., vol. 44, no. 7, pp. 1069-1074, 2016.

[97] E. C. Jordan and K. G. Balmain, Electromagnetic Waves and Radiation Systems, $2^{\text {nd }}$ Edition, p. 273, Prentice Hall, 1969.

[98] C. Chua, S. Aditya, and Z. Shen, "Planar helix with straight-edge connections in the presence of multilayer dielectric substrates," IEEE Trans. Electron Devices, vol. 57, no. 12, pp. 3451-3459, 2010.

[99] R. M. K. and P. P. Toulios, "Integrated circuits for the millimeter through optical frequency range,” Proc. Symp.submillim. Waves, p. 497-516., 1970.

[100] R. K. Arora, "Surface Waves on a Pair of Parallel Undirectionally Conducting Screens," IEEE Trans. Antennas Propag., vol. 14, no. 6, pp. 795-797, 1966.

[101] S. Aditya and R. K. Arora, "Guided Waves on a Planar Helix," IEEE Trans. Microw. Theory Tech., vol. 27, no. 10, pp. 860-863, 1979.

[102] C. G. Montgomery, R. H. Dicke, and E. M. Purcell, Principles of Microwave Circuits. Institution of Electrical Engineers, vol. 25. London, U.K.: Peter Peregrinus Ltd., 1987.

[103] Roger F. Harrington, Time-Harmonic Electromagnetic Fields, pp. 163-165, McGraw-Hill, 1961.

[104] S. Wang and S. Aditya, "Wideband Power Combining of Four Microfabricated WBand Traveling-Wave Tubes," IEEE Trans. Electron Devices, vol. 64, no. 9, pp. 3849-3856, 2017.

[105] C. Zhao, S. Aditya, and C. Chua, "A Microfabricated Planar Helix Slow-Wave Structure to Avoid Dielectric Charging in TWTs," IEEE Trans. Electron Devices, vol. 62, no. 4, pp. 1342-1348, 2015.

[106] S. T. Han, K. H. Jang, J. K. So, J. Il Kim, Y. M. Shin, N. M. Ryskin, S. S. Chang, and G. S. Park, "Low-voltage operation of Ka-band folded waveguide travellingwave tube," IEEE Trans. Plasma Sci., vol. 32, no. 1 I, pp. 60-66, 2004.

[107] V. L. Bratman, A. E. Fedotov, P. B. Makhalov, and V. N. Manuilov, "Design and numerical analysis of W-band oscillators with hollow electron beam," IEEE Trans. Electron Devices, vol. 61, no. 6, pp. 1795-1799, 2014.

[108] F. S. Rusin, "Orotron - an Electronic Oscillator with an Open Resonator and Reflecting Grating," Proc. IEEE, vol. 57, no. 4, pp. 720-722, 1969. 
[109] S. S. Ponomarenko, S. A. Kishko, V. V. Zavertanniy, E. M. Khutoryan, I. V. Lopatin, B. P. Yefimov, and A. N. Kuleshov, "400-GHz continuous-wave clinotron oscillator," IEEE Trans. Plasma Sci., vol. 41, no. 1, pp. 82-86, 2013.

[110] M. Sattorov, E. Khutoryan, K. Lukin, O. Kwon, and G. S. Park, "Improved efficiency of backward-wave oscillator with an inclined electron beam," IEEE Trans. Electron Devices, vol. 60, no. 1, pp. 458-463, 2013.

[111] C. B. Wharton and J. M. Butler, "Relativistic O-type oscillator-amplifier systems," Proc. SPIE Conf. Intense Microwaves Part. Beams, vol. 1226, pp. 23-35, 1990.

[112] V. A. Solntsev, "Carcinotrode - the BWO with the automodulation of the cathode emission," 10th Int. Conf. Vac. Microelectron., pp. 730-734, 1997.

[113] G. Barreto and C. B. Wharton, "Experimental Results from a Tandem BWO-TWT System Used to Generate High-Power Microwaves," IEEE Trans. Plasma Sci., vol. 20, no. 5, pp. 493-498, 1992.

[114] Y. N. Pchelnikov and V. A. Solntsev, "BWO with an amplifying section," Fifth IEEE Int. Vac. Electron. Conf. (IEEE Cat. No.04EX786), pp. 73-74.

[115] A. V. Haeff, "The Electron-Wave Tube-A Novel Method of Generation and Amplification of Microwave Energy," in Proceedings of the IRE, vol. 37, no. 1, pp. 4-10, Jan. 1949.

[116] P. M. Phillips, E. G. Zaidman, H. P. Freund, A. K. Ganguly, and N. R. Vanderplaats, "Review Of Two-Stream Amplifier Performance," IEEE Trans. Electron Devices, vol. 37, no. 3, pp. 870-877, 1990.

[117] A. W. Cross, A. J. MacLachlan, C. W. Robertson, L. Zhang, K. Ronald, and A. D. R. Phelps, "Highly overmoded MM-wave oscillator experiments," 12th UKEurope-China Workshop on Millimetre Waves and THz Technologies (UCMMT 2019), Queen Mary University of London, 2019.

[118] S. Li et al., "Preliminary experimental investigations into an oversized coaxial relativistic klystron amplifier at $\mathrm{Ka}$ band," 2019 International Vacuum Electronics Conference (IVEC), Busan, Korea (South), 2019, pp. 1-2.

[119] I. Lebedev, Microwave Engineering, Mir Publishers, Moscow, 1973. 


\section{Appendix A: Interaction Impedance}

Interaction impedance was introduced by Pierce [36]. It is defined as:

$$
K_{c, n}=\frac{\left|V_{z, n}\right|^{2}}{2 \mathrm{P}}
$$

where $V_{z, n}$ is the longitudinal voltage for $n^{\text {th }}$ space harmonic and $\mathrm{P}$ is the total time averaged RF power flowing through the SWS. Consider the longitudinal electric field for $n^{\text {th }}$ space harmonic in the SWS, $E_{z, \mathrm{n}}(r, \phi, z, t)$, as follows:

$$
E_{z, \mathrm{n}}(r, \phi, z, t)=\left|E_{z, \mathrm{n}}(r, \phi)\right| e^{j\left(\omega t-\beta_{\mathrm{n}} z-\varphi_{0, \mathrm{n}}\right)}
$$

where $\left|E_{z, \mathrm{n}}(r, \phi)\right|$ is the magnitude of $E_{z, \mathrm{n}}(r, \phi, z, t)$ at beam position $(r, \phi), \varphi_{0, \mathrm{n}}$ is the phase of $E_{z, \mathrm{n}}(r, \phi, z, t)$ at $z=0$ and $t=0$, and $\beta_{\mathrm{n}}$ is the phase constant of $E_{z, \mathrm{n}}(r, \phi, z, t)$. We obtain the longitudinal voltage $V_{z, n}$ by taking the negative line integral of the real part of the longitudinal electric field between the limits $\mathrm{z}=\frac{\lambda_{g}}{4}-\frac{\varphi_{0, \mathrm{n}}}{\beta_{\mathrm{n}}}$ where the field intensity is zero (at $\mathrm{t}=0$ ), and $\mathrm{z}=-\frac{\varphi_{0, \mathrm{n}}}{\beta_{\mathrm{n}}}$ where the field intensity is maximum [119]:

$$
\begin{gathered}
V_{z, n}=-\int_{z=\frac{\lambda_{g}}{4}-\frac{\varphi_{0, \mathrm{n}}}{\beta_{\mathrm{n}}}}^{-\frac{\varphi_{0, \mathrm{n}}}{\beta_{\mathrm{n}}}} \operatorname{Re}\left(E_{z, \mathrm{n}}(r, \phi, z, t=0)\right) d z \\
V_{z, n}=-\int_{z=\frac{\lambda_{g}}{4}-\frac{\varphi_{0, \mathrm{n}}}{\beta_{\mathrm{n}}}}^{-\frac{\varphi_{0, \mathrm{n}}}{\beta_{\mathrm{n}}}}\left|E_{z, \mathrm{n}}(r, \phi)\right| \cos \left(\beta_{\mathrm{n}} z+\varphi_{0, \mathrm{n}}\right) d z=\frac{\left|E_{z, \mathrm{n}}(r, \phi)\right|}{\beta_{\mathrm{n}}}
\end{gathered}
$$

The magnitude of $V_{z, n}$ is obtained as:

$$
\left|V_{z, n}\right|=\frac{\left|E_{z, \mathrm{n}}(r, \phi)\right|}{\beta_{\mathrm{n}}}
$$

Hence,

$$
K_{c, n}=\frac{\left|E_{z, \mathrm{n}}(r, \phi)\right|^{2}}{2 P \beta_{n}^{2}}
$$




\section{Appendix B: Calculation of Fourier coefficients}

clear all;

phase $=90 ; \%$ phase between 0 and 180

$\mathrm{D} 1=\mathrm{xlsread}($ 'Realdata.xlsx'); \% Data of real part is sored in an excel file named Realdata

$\mathrm{D} 2=$ xlsread('Imagdata.xlsx'); \% Data of imaginary part is sored in an excel file named Imagdata

$\mathrm{Z}=\mathrm{D} 1(:, 1)$

$\mathrm{Z}=\mathrm{Z} * 10^{\wedge}-3$;

$1=$ length $(Z)$;

$\operatorname{pitch}=\max (Z)-\min (Z)$;

if $(\bmod (1,2)==1)$

$\mathrm{ex}=0$;

else

$\mathrm{ex}=1$;

end

$\mathrm{n} 1=[\operatorname{ceil}(-1 / 2):$ floor(1/2)-ex $]$;

betan $=\left((\right.$ phase $*($ pi $/ 180))+\left(2 *\right.$ pi $^{*}$ n1 $\left.)\right) /$ pitch;

$\mathrm{RE}=\mathrm{D} 1(:, 2)$;

$\mathrm{IM}=\mathrm{D} 2(:, 2)$;

$\mathrm{F}=\operatorname{complex}(\mathrm{RE}, \mathrm{IM})$;

for $\mathrm{i}=1: 1$

$\mathrm{S}=\exp (\operatorname{complex}(0, \operatorname{betan}(\mathrm{i}) * \mathrm{Z}))$

$\operatorname{Ema}(\mathrm{i})=(1 /$ pitch $) * \operatorname{trapz}\left(\mathrm{Z}, \mathrm{F} .{ }^{*} \mathrm{~S}\right)$;

end

$\operatorname{stem}(n 1, a b s(E m a), ' b ')$; 


$$
\begin{aligned}
& E_{x i}=\frac{-j \omega \mu_{0}}{k_{C i}^{2}} \frac{d H_{z i}}{d y}+\frac{1}{k_{C i}^{2}} \frac{d^{2} E_{z i}}{d z d x} \\
& E_{y i}=\frac{j \omega \mu_{0}}{k_{C i}^{2}} \frac{d H_{z i}}{d x}+\frac{1}{k_{C i}^{2}} \frac{d^{2} E_{z i}}{d z d y} \\
& H_{x i}=\frac{j \omega \varepsilon_{0} \varepsilon_{r i}}{k_{C i}^{2}} \frac{d E_{z i}}{d y}+\frac{1}{k_{C i}^{2}} \frac{d^{2} H_{z i}}{d z d x} \\
& H_{y i}=\frac{-j \omega \varepsilon_{0} \varepsilon_{r i}}{k_{C i}^{2}} \frac{d E_{z i}}{d x}+\frac{1}{k_{C i}^{2}} \frac{d^{2} H_{z i}}{d z d y}
\end{aligned}
$$

where, $\quad k_{C i}^{2}=\omega^{2} \varepsilon_{r i} \varepsilon_{0} \mu_{0}-\beta_{n}^{2}$

$$
\begin{aligned}
& E_{y 1}=\sum_{n=-\infty}^{\infty}\left[\frac{-j \omega \mu_{0}}{k_{C}^{2}} u_{n} C_{n} e^{-u_{n}(x-a)}+\frac{1}{k_{C}^{2}} \frac{2 \pi n}{p_{y}} \beta_{n} A_{n} e^{-u_{n}(x-a)}\right] e^{\left\{j \frac{2 \pi n y}{p_{y}}\right\}^{\left\{-j \beta_{n} z\right\}}} \\
& E_{y 2}=\sum_{n=-\infty}^{\infty}\left[\frac{j \omega \mu_{0}}{k_{C}^{2}} u_{n} D_{n} \sinh \left(u_{n} x\right)+\frac{1}{k_{C}^{2}} \frac{2 \pi n}{p_{y}} \beta_{n} B_{n} \cosh \left(u_{n} x\right)\right] e^{\left\{\frac{j \pi n y}{p_{y}}\right\}^{\left\{-j \beta_{n} z\right\}}} \\
& H_{y 1}=\sum_{n=-\infty}^{\infty}\left[\frac{j \omega \varepsilon_{0}}{k_{C}^{2}} u_{n} A_{n} e^{-u_{n}(x-a)}+\frac{1}{k_{C}^{2}} \frac{2 \pi n}{p_{y}} \beta_{n} C_{n} e^{-u_{n}(x-a)}\right] e^{\left\{j \frac{2 \pi n y}{p_{y}}\right\}} e^{\left\{-j \beta_{n} z\right\}} \\
& H_{y 2}=\sum_{n=-\infty}^{\infty}\left[\frac{-j \omega \varepsilon_{0}}{k_{C}^{2}} u_{n} B_{n} \sinh \left(u_{n} x\right)+\frac{1}{k_{C}^{2}} \frac{2 \pi n}{p_{y}} \beta_{n} D_{n} \cosh \left(u_{n} x\right)\right] e^{\left\{j \frac{2 \pi n y}{p_{y}}\right\}^{\left\{-j \beta_{n} z\right\}}} \\
& E_{x 1}=\sum_{n=-\infty}^{\infty}\left[\frac{\omega \mu_{0}}{k_{C}^{2}} \frac{2 \pi n}{p_{y}} C_{n} e^{-u_{n}(x-a)}+\frac{j}{k_{C}^{2}} \beta_{n} u_{n} A_{n} e^{-u_{n}(x-a)}\right] e^{\left\{j \frac{2 \pi n y}{p_{y}}\right\}} e^{\left\{-j \beta_{n} z\right\}} \\
& E_{x 2}=\sum_{n=-\infty}^{\infty}\left[\frac{\omega \mu_{0}}{k_{C}^{2}} \frac{2 \pi n}{p_{y}} D_{n} \cosh \left(u_{n} x\right)-\frac{j}{k_{C}^{2}} \beta_{n} u_{n} B_{n} \sinh \left(u_{n} x\right)\right] e^{\left\{j \frac{2 \pi n y}{p_{y}}\right\}^{\left\{-j \beta_{n} z\right\}}} \\
& H_{x 1}=\sum_{n=-\infty}^{\infty}\left[\frac{-\omega \varepsilon_{0}}{k_{C}^{2}} \frac{2 \pi n}{p_{y}} A_{n} e^{-u_{n}(x-a)}+\frac{j}{k_{C}^{2}} \beta_{n} u_{n} C_{n} e^{-u_{n}(x-a)}\right] e^{\left\{j \frac{2 \pi n y}{p_{y}}\right\}^{\left\{-j \beta_{n} z\right\}}} \\
& H_{x 2}=\sum_{n=-\infty}^{\infty}\left[\frac{-\omega \varepsilon_{0}}{k_{C}^{2}} \frac{2 \pi n}{p_{y}} B_{n} \cosh \left(u_{n} x\right)-\frac{j}{k_{C}^{2}} \beta_{n} u_{n} D_{n} \sinh \left(u_{n} x\right)\right] e^{\left\{j \frac{2 \pi n y}{p_{y}}\right\}^{\left\{-j \beta_{n} z\right\}}}
\end{aligned}
$$




\section{Appendix D: MATLAB programme for finding the discrete Fourier transform of BWO output signal}

$\%$ clc;clear all;

$\%$ Command to select, and read the .txt file

[name,path,check]=uigetfile('.txt','import data'); \% To read txt data

$\mathrm{T}=$ importdata(fullfile(path,name));\% To read txt data

$\mathrm{u}=\mathrm{T}$.data(:,2);\% Select the amplitude vaues, T.data(:,1) has time steps

Inputsig $1=\mathrm{u}(30276: 60551) ; \%$ Considering the input signal at required time window

\%starting and ending of time period (30276-50ns 60551-100ns 90827-150 ns

\%121102-200ns 151377-250 ns 205ns-124129, etc...)

$\mathrm{N}=\operatorname{size}($ Inputsig 1$) ; \%$ length of data points

$\mathrm{Fs}=605500000000 ; \%$ fs calculated from sampling time of signal from PIC solver

$\%$ FFT calculation

FFT1 $=\operatorname{abs}(\mathrm{fft}($ Inputsig1,N)$) \cdot / \mathrm{N}$;

Fourier $=$ FFT1(1:ceil(N/2)); \%Finding one side of the spectrum

\%Finding $\mathrm{X}$-axis of Fourier transform

figlen=length(Fourier);

freq_FT $=(0:($ figlen -1$)) *(\mathrm{Fs} /(2 *$ figlen $))$;

\%Ploting Fourier transform in linear scale

\%plot(freq_FT/(1e9), Fourier); \%Plot in linear scale

\%Ploting Fourier transform in $\mathrm{dB}$ scale

plot(freq_FT/(1e9),20.*log10(Fourier)); 